West Virginia University Libraries

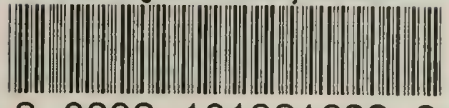

308021019316822 


\section{- \\ UCT 2?:1964}

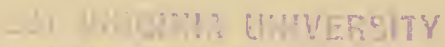

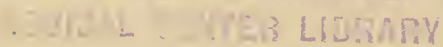

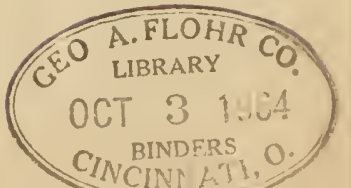




\section{OLD BOOKS}

Q143

P26b

I8ZR 18.74 


Digitized by the Internet Archive in 2010 with funding from

Lyrasis Members and Sloan Foundation 


\section{BLARINGHEM}

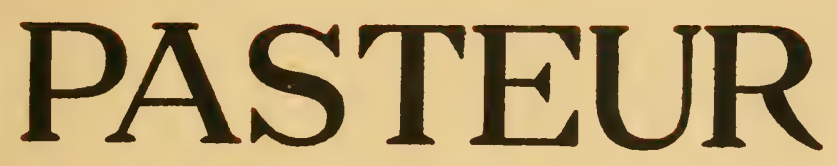

(t)

\section{LE TRANSFORMISME}

PRÉFACE DE J.COSTANTIN

MASSON $U$ CIE PARIS 



\section{PASTEUR}

ET

LE TRANSFORMISME 


\section{A LA MEME LIBRAIRIE}

(EUVRES I)E PASTEUR

Les Envres de Pasteur seront complètes en 7 volnmes, in-8० jésus, qui paraitront successivement. Le prix de chaque volume sera fixé au moment de sa publication et d'après son importance. Chaque volume se vendra séparément.

Volumes parus :

Tome I. - Dissymétrie Moléculaire. 1 volume de 480 pages avec nombreuses figures dans le texte et 1 portrait hors texte représentant Pasteur dans son laboratoire. Prix : 50 francs.

TOME II. - FErdentations ET Générations dites spontanées. 1 volume de 660 pages arec nombreuses figures dans le texte. Prix : 65 francs. 


\section{BLARINGHEM}

Maître de Conférences à l'Ecole Normale supérieure Chef de Service à l'Institut Pasteur

\section{PASTEUR}

ET

\section{LE TRANSFORMISME}

Avec une préface de M. J. COSTANTiN Memhre de l'Institut

MASSON ET Cie, ÉDITEURS LIBRAIRES DE L'ACADÉMIE DE MÉDECINE 120. Boulevard Saint-Germain, PARIS (VIO) 1923 


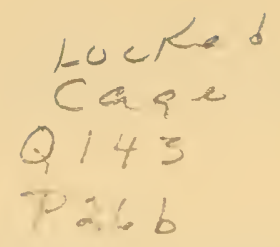

Tous droits de reproduction, de traduction et d'adaptation réservés pour tous pays.

Copyright 1923 by Masson et $C^{\text {ta. }}$ 


\section{AUX JELNES NORMALIENS}

j'offre ces conférences; je demande à tous un peu d'attention, à certains des critiques, à la plupart de l'enthousiasme.

Il y a soixante-quinze ans, le jeune Lovis PASteur, élève respectuelix du grave DeLAfosse, entraîné par le bouillant BALARD, a pris passion, dans les laboratoires et les bibliothèques de l'Ecole, pour le contrôle des faits enseignés; bientôt, solls linfluence d'un esprit original, Auguste LAUnent, son génie s'éveille et construit de larges hypothèses, poursuit l'enchaînement logique de leurs conséquences, qu'il vérifie avec patience dans leurs moindres détails.

Dix ans plus tard, conscient de sa valeur, PASTEur, déjà célèbre, revient à l'Ecole, découvre et entraine à sa suile J. Raulix (1860), E. Ducladx (1862), Ph. van Tieghem (1862), D. Gernez (1865), E. Maillot (1868), tolls savants dont il fit des émules en lenr laissant deviner ses projets, ses dontes, ses espoirs.

Enfin, dans le triomphe de ses conceptions et de ses méthodes, il groupe antour de lui U. GAYON (1874), F. GRENET (1875), dont il fait des directeurs de services techniques, puis Jolbert (1876), C. Chamberland (1878), M. le Doctelir Roux (1878), L. Thulllier (1880), E. Nocard (1887)..., qui, inspirés par le Maître, perfectionnent les théories, consacrent leur vie et lenr carrière au développcment et allx applications de la nouvelle doctrine.

J'ai eul l'honneur de suivre les enseignements de plusieurs 
de ces chefs de l'Ecole pastorienne, d'être accueilli par $M$. le Docteur Roux, par Elie Metchnikoff (1908), dans la maison qui n'a pas failli à la lourde charge de poursuive des travaux dignes de son fondateur. Je vons invite à continuer la tradition née à l'Ecole Normale et d'accepter la règle si rigonreusement obscrvée par PASTEUR :

Apprendre, contrôler avec soin, laisser à l'imagination la plus large indépendance et ne manquer jamais une occasion de servir.

Ecole Normale, 27 décembre 1922. 


\section{PRÉFACE}

Pasteur, chargé par J.-B. Dumas d'écrire une introduction aux nuvres de Lavolsier, qu'ou réimprimait, s'exprimait ainsi : "C'est dans la lecture des travanx des inventeurs que la flamme sacrée de l'invention s'allume et s'entretient. " M. Blaringhem n'a pas attendu l'apparition der sept volumes des ouvres complètes de Pasteur, dont denx volumes sont déjà publiés, pour en entreprendre la lecture et l'étude aux sources originales : il a été très heureusement inspiré, car tont ce qu'a pensé ce puissant esprit devra être l'objet des méditations des générations à venir.

Le titre du présent volume de M. Blarivghem surprendra évidemment beaucoup de lecteurs : PASTEUR ne s'est jamais occupé du transformisme, et pent-être même a-t-il envisagé, à certaines heures, cette doctrine avec défiance. Cependant, il est incontestable que ses grandes déconvertes sur la vaccination, sur les variations de la virulenee des Bactéries et sur les changements de la résistance des organismes supérieurs à leurs attaques constituent des documents de premier ordre pour la conception évolutionniste. Cette question est traitée avec ampleur dans l'ouvrage que le brillant biologiste offre anjourd'hni au public, mais on $\mathrm{y}$ tronve beau- 
coup d'autres idées très originales qui passionneront certainement bon nombre d'esprits.

Les recherches de Pasteur sur la dissymétrie moléculaire occupent une large place dans le livre actuel; c'est que le naturaliste peut tirer le plus grand enseignement de la vie des cristaux, de leur dimorphisme ou polymorphisme, de leurs cicatrisations, de leurs régénérations. Parmi les curiosités de cette étude, je signalerai les homologies qui tendent à foumir une explication des chimères de Morelle et de Tomate de WinckLer, ainsi que des lois numériques de MFNDEL et de l'hérédité alternante. Il y a lieu également d'attirer l'attention du lecteur sur l'histoire des lyybrides cristallins et sur la figure tirée du mémoire de Pasteur (p. 72), où la mosaïque s'accuse dans la cristallisation du bimalate d'ammoniaque et du bitartrate d'ammoniaque, fait qui jette une lueur sur les mosaïques signalées autrefois par Naudin dans le règne végétal, étudiées également avec perspicacité par MT. BLaringhem.

Mais c'est surtout la notion des germes cristallins qui offre un grand intérêt. Leur rôle a été mis en évidence par les beaux travaux de Gernez, un élève de Pasteur, dont la profonde originalité n'a pas toujours été appréciée comme clle le méritait. M. Braringhem remarque, à ce sujet, que les sels susceptibles d'être maintenus en état de sarsaturation se comportent comme des éléments femelles dans l'attente de l'unique germe (anthérozoïde, grain de pollen) nécessaire et suffisant pour former l'œuf et déclencher son développement en embryon (Decaisne et THuret, 1844). Le cristal-germe de formiate de strontiane épuise la solution 
inactive de ce corps, et selon que le cristal ensemencé possède l'hémiédrie droite on gauche, provoque la cristallisation du formiate droit ou gauche. "Il fournit, dit MI. BLARINGHEM, une démonstration très élégante du fait découvert par PASTEUR quinze ans plus tôt, à savoir que, chez le formiate de strontiane, la dissymétrie n'est qu'apparente ; elle affecte l'arrangement des moléeules et non la molécule elle-même, car les solutions sont inactives " (en lumière polarisée).

On saisit par cet exemple la eomplexité du problème de la dissymétrie moléculaire. Le cas de l'hémiédrie des tartrates et de l'inactivité des racémates (ou paratartrates) était autre. On sait que lorsque PASTEur fit, en étudiant ces sels, sa première découverte, son saisissement fut tel qu'il ne put remettre l'œil à l'appareil de polarisation. Il s'élança brusquement hors du laboratoire de BALARD où il travaillait et s écria comme ARCHIMÈDE : "Tout est trouvé ", et, rencontrant un préparateur de Physique dans le corridor de l'Ecole Normale, il l'embrassa.

Il arait constaté que les racémates étaient formés d'un mélange de cristaux hémiédriques droits et ganches et, les ayant triés à la pince, il put isoler les hémiédriques gauches (qui étaient alors inconnus), et rérifier, ce qu'il prévoyait, qu ils devaient dévier à gauehe.

On se rappelle avec quelles précautions minutieuses et défiantes Вют lui fit répéter sous ses yeux l'expérience, et, quand l'exactitude en fut vérifiée, l'illustre vieillard s'écria : " Mon cher enfant, j'ai tant aimé les sciences dans ma vie, que cela me fait battre le cœur. ") Ces paroles, où la passion cependant contemue du savant se trahissait avec intensité, 
s'expliquent parce qu'il voyait, avec émotion et avec joie, ses prévisions réalisées : ces souvenirs ne devaient jamais s'effacer' de la mémoire de PASTEUR.

Dans l'histoire de cette première trouvaille, on assiste à la démar'che de l'esprit en quête de la vérité : l'étude ardente des faits' connus, puis l'intervention des idées préconçues, corrélation possible de l'hémiédrie et du pouvoir rotatoire, rôle immense que jonent ces phénomènes dans la conception de la matière; enfin travail acharné de l'expérimentateur pour contrôler la justesse de ses prévisions.

Plus tard, il apprend que chez un fabricant de produits chimiques d Allemagne le tartrate de chaux impur des fabriques, souillé de matières organiques et abandonné sous l'earu, en été, peut fermenter et donner divers produits ; il est amené naturellement à étudier cette question. C'est ainsi qu il fut conduit à l'examen des fermentations, problème qui, par l'attirance des questions insolubles, devait l'entrainer vers celni des générations spontanées, des germes vivants, des germes infectieux, sources de presque toutes les maladies contagieuses.

Il met le paratartrate d’ammoniaque en présence de la Levure, agent du phénomène précédent, et il s'aperçoit avec surprise qu'elle opérait merveilleusement la séparation faite par lui devant Bıot, au Collège de France : elle dévore le tartrate droit et laisse inattaqué le ganche, aussi le liquide primitivement neutre au point de vue rotatoire, inactif par conséquent, aequiert peu à peu un ponvoir rotatoire gauche, qui augmente jusqu'à un maximum; la fermentation est alors suspendue et la liqueur peut fournir une belle cristal- 
lisation de tartrate gauche indécomposé. Donc la Levure connaît la dissymétrie moléculaire. Cela n'étonne pas Pasteur, car " au moment de l'élaboration de l'organisme végétal une force dissymétrique est présente ".

" Tout phénomène de nutrition est protoplasmique, dit Duclaux, c'est-à-dire que l'aliment d'une cellule quelconque, quel qu'il soit et quelle qu'elle soit, doit commencer par faire partie du protoplasma avant d'être brûlé ou utilisé. "

"On trouve, dit Pasteur, la dissymétrie établie dans un très grand nombre de principes immédiats animaux et régétaux, notanment dans les principes immédiats de la vie. Tous les produits, pour ainsi dire, de l'ouf et de la graine sont dissymétriques. " "Une cellule vivante nous apparaît comme un laboratoire de forces dissymétriques où un protoplasma dissymétrique agit sous l'influence du soleil, c'està-dire sous l'infinence de forces extérieures dissymétriques. ॥

Pasteur ajoute : "Ces forces dissrmétriques, placées peut-être sous des influences cosmiques, résident-elles dans la lumière, dans l'électricité, daus le magnétisme, dans la chaleur? Seraient-elles en relation avec le mourement de la terre, avec les courants électriques, par lesquels les phỵsiciens expliquent les pôles magnétiques terrestres ? Il n'est pas possible aujourd'hui d'émettre à eet égard les moindres conjectures. ")

"Si l'influence mystérieuse à laquelle est due la dissymétrie des produits naturels venait à changer de sens ou de direction, les éléments constitutifs de tous les êtres vivants prendraient une dissymétrie inverse. Peut-être un monde nouveau s'offrirait à nous. Qui pourrait prévoir l'organi- 
sation des êtres vivants si la cellulose, de droite qu'elle est, devenait gauche, si l'albumine du sang devenait droite? I] y a là des mystères qui préparent à l'avenir d'immenses travaux et appellent, dès anjourd'hui, les plus sérieuses méditations de la science. )

Visions extraordinaires qui montrent bien que Pasteur était, au moins par sa pensée andacieuse, le plus révolutionnaire des transformistes,

Ces conceptions, il y a songé jusqu'à sa dernière heure, comme celles qui étaient nées dans son esprit pendant sa jeunesse et auxquelles il tenait fortement. D'antres questions grandioses s'emparèrent de sa pensée, à partir de 1859 : maladie des vers à soie et, plus tard, maladies contagieuses de lhounme et des animanx supérienrs.

Une fois engagé sur cette voie nouvelle, par une force invincible, sa puissante intelligence fut conduite sans arrêt de la dissymétrie moléculaire à la vaccination de la rage. Cependant, chaque fois que, pour une cause accidentelle, la guerre de 1870 , par exemple, sa pensée est obligée de se reposer, on voit reparaître les préoccupations premières de son esprit. Il écrit à Duclaux, en mars 1871 : "Ah ! que ne suis-je riche, millionnaire, je vous dirais à vous, à RAULIN, à GERnez, à VAN TiegheM, venez! nous allons transformel le monde par nos déconvertes. ") Evidemment, si ce dernier nom est venu an bout de sa plume, c'est qu'il sentait le besoin d'avoir près de hi un naturaliste pour tout ce qu'il voulait entreprendre.

Il a songé à cet instant à des expériences qui n'ont pas donné de résultat et qu'il n’a plus eu le temps de recom- 
mencer plus tard. "Que faut-il faire pour imiter la nature ? Il faut rompre avec vos inéthodes, qui sont à ce point de rue surannées et impuissantes. Il fant chercher à faire agir des forces dissymétriques, recourir à des actions de solénoïdes, de magnétisme, de mouvement dissymétrique lumineux, à des actions de substances elles-mêmes dissymétriques. ")

Nous retrouvons les traces des mêmes préoceupations dans me conversation avec Chamberland, au cours d'une promenade dans le jardin du Luxembourg. J'ai eu, nnoimême, l'écho de ces pensées par un de nos élèves de la section des sciences naturelles de l'Ecole Normale, LE DANTEC, qui était devenu son préparateur et qui avait su lui inspirer une grande sympathie et une grande confiance, de sorte qu'il hii montrait ses anciens cahiers de notes et d'expériences et qu ìl lui expliquait ces notions si séduisantes pour un naturaliste d'élite comme celui-là.

En 1876 ou 18Ti, au moment où il allait s'engager sur la piste passionnante du charbon, il condensait ses notes sur la dissymétrie moléculaire, en vue de la publication d'un grand ouvrage qui n'a jamais vu le jour. Le 22 décembre 1883, il faisait sur ce sujet une conférence à la Société chimique de Paris.

On peut done dire, malgré les apparences contraires, que, jusqu à sa dernière heure, Pasteur est resté le transformiste que nous envisagions plus haut.

Il est certainement regrettable pour la science qu'il n'ait pas mis au service de ce grand problème de l'évolution sa dialectique redoutable et la puissance incomparable d'un 
expérimentateur qui n'a pas eu d'égal. Mais, pour le grand bien de l'humanité souffrante, il orienta sa pensée vers l'étude des fermentations dont l'explication, comme l'avait dit Boyle déjà depuis deux cents ans, devait donner la clef de l'énigme des fièvres et des maladies contagieuses : prophétie singulière mais inerte et stérile d'un savant anglais un peu oublié qui devait, grâce à PASTEUR, devenir le verbe de l'avenir.

L'ouvrage si intéressant et si vivant de M. BLARINGHeM contribuera puissamment à faire connaître des aspects nouveanx ou insuffisamment connus de l'œuvre immense de Pasteur ; il y a tout lieu de penser qu'il servira à orienter les sciences biologiques sur des voies nouvelles et fécondes.

J. Costantin. 


\section{PASTEUR ET' LE TRANSFORMISME}

\section{LIVRE PREMIER \\ LES VARIATIONS DE L'ESPÈCE}

\section{CHAPITRE PREMIER}

\section{LA NOTION D'ESPÉCE}

La classification des formes, minérales et organisées, date de Linné (1738-1778). Le naturaliste suédois s'occupa surtout des plantes, mais, aidé par ses contemporains, il édifia un système de nomenclature encore adopté universellement et une méthode de distribution des types naturels qui présente des progrès immenses sur les tentatives honorables de Césalpin (1583), des Bauhin, Morison, Ray et Tournefort. La valeur de cette méthode repose uniquement sur l'application de trois principes qui dominent actuellement encore les données de la biologie :

"Nous comptons autant d'espèces qu'il y a eu, au commencement, de formes diverses créées (Fundamenta botanica, 1736) ;

"Le méthodiste ne se préoccupe pas des variétés les plus légères (1751);

"Les formes sexuelles constituent à elles scules les variétés naturelles; toutes les antres sont des monstres (1751). »

Appliquant ces principes à la répartition des végétaux, puis des animanx et des minéraux, Linvé édifia un Système de la Nature dont l'influence se fit sentir au cours d'un 
siècle. Les maîtres de Pastevr, et Pasteur lui-même, n'ont pu s'en dégager complètement et la notion de la constance du type originel domine l'œurre pastorienne.

Le succès de la méthode de Linné tient pour une bonne part à l'adoption, universelle, de la nomenclature binaire. Les espèces y sont désignées par deux noms latins; le pre. mier donne le genre et correspond souvent au nom vulgaire ; le second est la véritable définition de l'espèce, dont on trouve la signification et les limites dans les Flores, les Faumes, les Catalogues de minéraux; à la longue description des caractères distinctifs difficilement condensés sous la forme de diagnoses, LrNné substitua un seul nom; l'usage veut que le premier nom d'espèce donné à une forme décrite, ou figurée, ou représentée par des échantilions dans les collections célèbres, soit le nom définitif de tout le groupe d'êtres ou de matériaux qui lui ressemblent. On peut done dire que l'espèce systématique date de Linsé et sa définition doit être prise à la lettre :

"Autant on rencontre anjourd'hui de formes ou d'organisations différentes, autant il existe d'espèces primitives et perpétuelles; chacune des formes actuelles dérivant d'une de celles que l'Etre infini a initialement produites et qui ont subsisté à travers les temps, toujours semblables à ellesmêmes. "(Philosophica botanica, 1751.)

L'espèce systématique renferme done la suite des individus nés les uns des autres, toujours semblables, de plus en plus nombreux. Dans l'intérêt du progrès des sciences descriptives, il y a lieu de conserver cette définition. Tout groupe méritant une place dans la nomenclature binaire doit répondre à la fois anx conditions de perpétuation par la reproduction sexuelle et de similitude des caractères précisés par les diagnoses des Catalogues. 
LiNNÉ a soutenu pendant trente ans la nécessité de ces règles conventionnelles; ses ouvrages ont acquis dans le monde entier assez de popularité pour que l'accord se soit fait entre tous les naturalistes descripteurs; les avantages d'une langue commode pour la démonstration et la belle ordonnance des cabinets, pour les échanges et les communications, firent plus pour aboutir à ce résultat que les plus longues dissertations. La définition de l'espèce systématique s'est imposée par son caractère pratique et par la nécessité dans laquelle on se trouvait d'adopter un langage universel, indépendant des théories et des hypothèses, à une époque où l'on faisait l'inventaire des richesses de la nature.

A vrai dire, Linné a eu le soupȩon d'une évolution dans le temps; mais elle n'apparaissait que sous la forme de monstres ou de ces variétés dont le méthodiste ne doit pas tenir compte. A l'Université d'Upsal, où il fut, à la fin de sa vie, entouré d'élèves et d'admirateurs, il provoqua la soutenance de nombreuses thèses, réunies dans les Amoenitates Academicae (1749-1790), où il n'est question que de l'origine des Froments, des Linaires peloriées, des hybrides, sujets étudiés actuellement par les génétistes; on y trouve (Amoenitates, édit. d'Erlangen, t. VI, p. 1) l'hypothèse émise par Linné lui-niême : "Toutes les espèces d'un même genre auraient constitué à l'origine une seule espèce $a b$ initio unam constituerint speciem; elles se seraient ensuite multipliées par des générations hybrides. ” KoeLreuter (1761), C. Sprengel, A. Knigtir et Herbert, ponr ne citer que les précurseurs, ont suivi Linsé dans la voie qui fut élargie par les contemporains de Louis PaSTEUR, je reux nommer Charles Naudin et Gregor Mendel.

Un correspondant français et ami de Linné, Bernard DE Jussieu, jardinier de Lonis $\mathrm{XV}$, orienta les méthodistes 
dans une antre voie. En déplaçant, pour mettre en évidence leurs affinités, les nombreuses plantes du Jardin de Trianon qui venait d'être créé, il réalisa une suite de formes qui présentaient d'indiscutables affinités. BERnaRd DE JUSSIEU négligea, du moins dans son travail original, les documents desséchés, matériaux d'herbier, gravures et descriptions incomplètes; il observa et classa des plantes vivantes, travail condensé sous la forme d'un Catalogne vers 1765 et origine de la Méthode naturelle.

Nous y trouvons la première application d'un principe directeur, la subordination des caractères, dégagé en quelque sorte de la nature même des objets étudiés. Cette découverte place à mon avis Bernard De Jussieu, le jardinier qui ne nous a laissé qu'un Catalogue, au premier rang parmi les philosophes du dix-huitième siècle : "Les caractères doivent être pesés et non comptés. "

Il existe dans les êtres vivants des suites de formes qui, par leur homogénéité, méritent le qualificatif de naturelles, les Graminées, les Composées, les Primates par exemple. En faisant le recensement des caractères les plus fréquents, puis des moins communs, on constate, après les avoir sériés selon leur fréquence relative, que certains d'entre eux en entraînent d'autres de rang inférieur, si bien que l'énoncé d'un earactère bien choisi et nettement délimité équivaut à l'exposé non seulement du caractère lui-même, mais de toute une série de particularités moindres qui y sont liées. La subordination des caractères est done l'analyse et la sériation par ordre d'importance des éléments dont l'assemblage constitue l'espèce, le genre, la famille, l'ordre, la classe. C'est aussi la démonstration de la permanence de certains éléments dans la suite supposée des transformations.

Vers 1775 , LavoISIER introduit la même idée fondamen- 
tale en chimie. Il établit une distinction capitale entre les corps pondérables, dont la masse ne change pas au cours des transformations, et les agents impondérables, chaleur, lumière, dont l'intervention modifie l'état, la forme. Il démontre, par l'emploi de la balance, que l'air intervient pour augmenter le poids des métaux calcinés, que certains éléments indécomposables, ou corps simples, subsistent intacts et que leur poids demeure constant. Il impose à l'esprit de tous le principe fondamental que " rien ne se perd et rien we se crée ".

Désormais la notion d'espèce chimique acquiert la préci. sion définitive. Les formules empiriques de Linvé et des méthodistes, qui représentaient les classes et les familles par une suite de symboles résumant le groupement des pièces constitutives des fleurs, prennent en chimie ume importance décisive ; la diagnose des corps bruts est remplacée par une formule de constitution qui traduit sous une forme condensée et pratique toutes les propriétés latentes et apparentes des dérivés qu'en retireront le laboratoire ou l'industrie. L'acquisition d'un principe directeur, la permanence du poids des corps simples contrôlable par un instrument précis, fut suivie de l'adoption d'une nomenclature concise mettant en évidence les proportions des corps entrant en combinaison ; la distinction décisive entre la matière pondérable et les agents de transformation, chalenr', électricité, lumière, provoqua dans les sciences physiques et naturelles une révolution profonde. Désormais, pour tous les savants, pour tous les philosophes, la stabilité de l'espèce suggère l'idée d'un édifice dont les matériaux peuvent être dispersés, mais qui subsistent, se transmettent en conservant leur individualité. L'hérédité des éléments de l'espèce est admise sans restric. tion. 
La cristallographie profita largement des idées de perma. nence qui s'imposèrent à l'esprit des savants du début du dix-neuvième sièrle. HauY imagina les molécules intégrantes, solides élémentaires dont la juxtaposition en nombre illimité donnait le cristal ; mais, comme le remarque judicieusement Duclaux, dans l'Histoire d'un Esprit, il n'était pas encore question d'identifier ces matériaux à la molécule chimique ; les formes des molécules intégrantes résultaient du groupement des molécnles chimiques; de ce groupement dérivait la forme définitive des cristaux et, par suite, les limites des classes dans lesquelles on les rangeait. Dans les systèmes d'HAuY, le groupement des particules identiques était commandé par la nature propre de l'espèce chimique et il fallait imaginer autant de charpentes que de corps distincts capables de cristalliser. En mettant en valeur les eas d'isomorphisme, MitscherLich ramena bientôt les différentes formes de molécules intégrantes à des groupements plus généraux. Les carbonates cristallisés de calcium, de magnésium, de fer, de manganèse, pouvaient se substituer l'un à l'autre dans un cristal, ou mieux, les atomes de calcium pouvaient être remplacés par un nombre égal d'atomes de magnésinm sans destruction de l'équilibre cristallin. Il y avait done une forme de la molécule intégrante indépendante pour une part de la composition chimique. D'ailleurs, tous les earbonates ne possèdent pas cette propriété ; les carbonates de baryum, de strontium eristallisent sous une autre forme. Dans l'ordre des carbonates, on est amené à distinguer un genre du type carbonate de calcium, un genre du type carbonate de baryum et dans ces genres des espèces.

L'espèce fut done définie par la molécule. Elle exigeait l'identité de nature des atomes simples constituants et l'identité du nombre absolu des atomes, en un mot l'identité pon- 
dérale. L’identité dans l'arrangement des atomes autour du centre de gravité de la molécule, ou l'édifice moléculaire même, avait une portée générale et comprenait une foule de substances différentes, de mêmes formes et pouvant se substituer les unes aux autres sans amener de désordre. Dans un pareil groupement ou genre, il entre des éléments indépendants, distincts, calcium, magnésium, fer, pouvant se remplacer sans détruire la forme et la cohésion de l'édifice. Nous tirerons parti de cette notion dans l'examen des conséquences des Règles mendéliennes de l'hérédité.

L'espèce comprend des variétés. Sous l'influence de conditions diver'ses, les molécules identiques peuvent se grouper pour donner des ensembles différant par la forme, la couleur, la transparence et d'autres propriétés physiques. D'or'dinaire, d'ailleurs, la pureté laisse à désirer ; sans admettre que le minéral ait perdu son individualité spécifinne, le naturaliste distingue ces altérations et, en fait, leur a souvent donné des noms différents.

Delafosse, le maitre vénéré de Pasteur, étndia spécialement une catégorie de ces variétés connues sous le nom de formes hémiédriques. Un cristal est limité par un ensemble de faces et les règles théoriques de la répartition des inolécules veulent que taute forme cristalline se présente avec le nombre complet de faces qui la caractérisent. Or, en 1819, Weiss avait reconnu des cas où une partie des faces faisait défaut; la disparition n'était pas quelconque; elle ne portait que sur certaines faces, toujours les mêmes; pour désigner les variétés de quartz, de tonurmaline où il ne se formait que la moitié des éléments prévus, Weiss avait créé le mot Hémiédrie. Delafosse fit connaître une autre catégorie de variétés, les formes tétartoédriques, où le nombre des faces 
était réduit an quart du nombre normal ; en exposant les résultats de ses travaux, il fournit des explications qui tendent à atténuer l'importance de l'identité de forme de la molécule intégrante et du cristal. PASTEUR complète cette distinction en reportant dans la molécule chimique les causes de cette discordance; il démontre (1818) l'existence d'une disşrmétrie moléculaire chez certaines espèces présentant tonjours l'hémiédrie.

\section{***}

Vers la même époque (1S46), un botaniste amateur lyoulnais, Alexis Jordan, introduisit en biologie nue notion nouvelle, celle des petites espèces. Laissant die côté les conceptions généralisatrices de ses devanciers et négligeant les données, admises à cette époque, de l'enchaînement naturel des formes classées selon la méthode de Bernard dE Jussieu, il se proposa de montrer, dans les espèces de Linné, l existence d'un nombre considérable de formes, parfaitement délimitées, constantes et invariables dans leurs différences, en un not, d'unités véritables qui, soules, répondent à la notion expérimentale de l'espèce.

Les Pensées sauvages constituent l'espèce Viola tricolor de la nomenclature binaire ; mais la diagnose ne correspond pas à la description d'un ensemble homogène d'individus; elle renferme des formes distinctes dont la constance, par voie de semis, fut vérifiée par JoRDAN durant plus de trente générations. Il en est de vivaces, à racines épaisses et à cotylédons pourvus d'iun véritable pétiole; d'autres sont annuelles à cotylédons sessiles. Pamni ces dernières, la forme agrestis a des tiges étalées, flexueuses, des fleurs de taille moyenne à pétales denticulés sur les bords (fig. 1, a) colorés 
en jaune lilas allant jusqu'au bleu ; la forme segetalis, à tiges très ramifiées porte des fleurs grandes (fig. 1, $s$ ) à
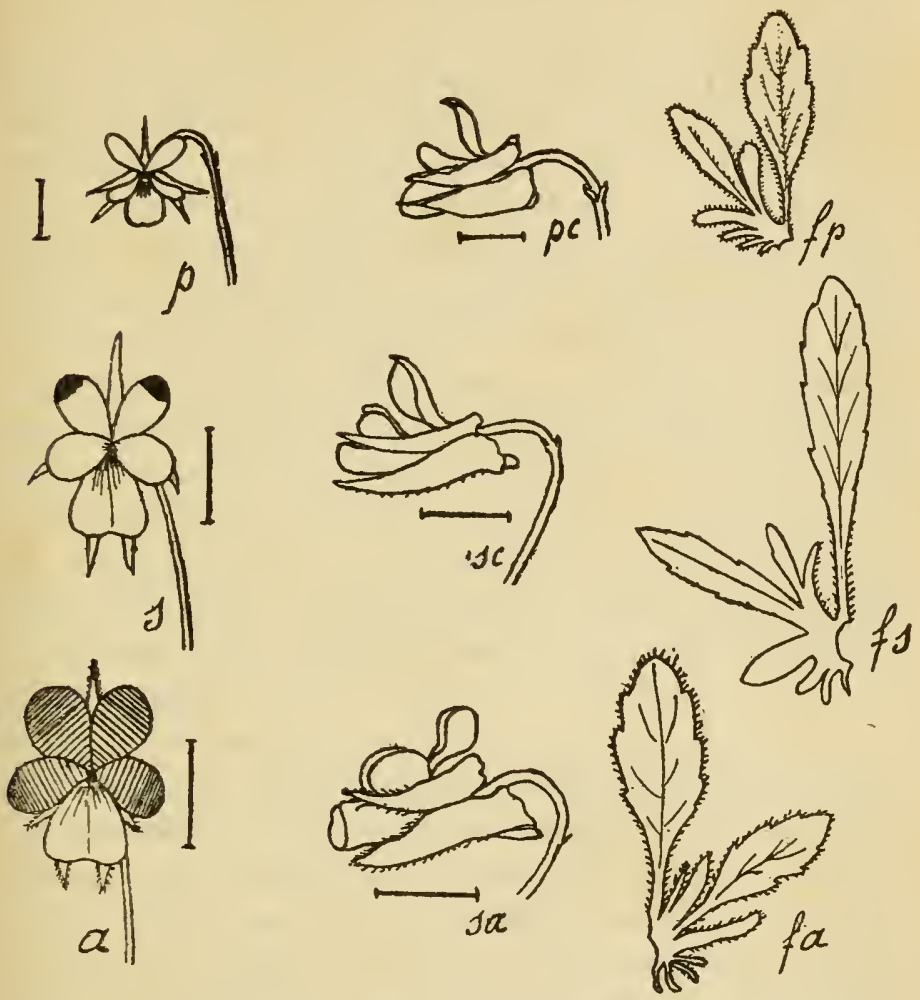

Fig. 1. - Petites espèces de Viola tricolor L.; $p$, fleur de la sous-espéce pallescens; $p c$, sépales ; $f p$, feuille avec une stipule ; $s$, fleur de la sous-espèce segelalis; a, fleur de la sous-espèce agrestis (d'après Cusix).

pétales jaunâtres, dont les supérieurs sont ornés de larges macules violettes nettement délimitées; la forme pallescens (fig. $1, p$ ) est tonjours grêle avec des fleurs petites, blanches 
et dépourrues de stries rayonnantes... Toutes ces formes se distinguent dans les cultures expérimentales lorsqu'on les sème côte à côte dans les mêmes plates-bandes. Lue port, les feuilles et les stipules, les calices et les fruits, sont caractéristiques et, à tout âge, mêne au début de la germination, un expérimentateur exercé réussit à les nommer correctement; elles conservent leur's caractères particulicrs par voie de semis, sans fournir de termes de transition.

JoRDAN énonce (i) les principes qui le gruident dans son travail : "On est généralement d’accord que les plantes qui présentent des caractères (différentiels) constants sont des espèces distinctes; en un mot, que la constance des carac. tères est la marque de l'espèce. ) Pour s'en assurer, l'observation directe et le semis sont la meilleure voie. " Il convient d "étudier encore les espèces roisines du même genle ou de genres rapprochés, celles surtout dont la constance n'est l'objet d'aucun doute et qui forment par leur réunion ce quion appelle des groupes naturels, afin d'e voir quelles sont les différences qui les séparent et de pouvoir faire ensuite la comparaison de leurs caractères avec ceux de la plante qu'on cherche à connaître. Si les caractères de celles-ci équivalent aux autres pour le nombre et l'importance, s'ils portent sur les mêmes organes, il sera déjà extrêmement probable, d'après le seul résultat de cette comparaison, qu'elle mérite d'être considérée comme une espèce véritable aux mêmes titres que les autres le sont elles-mêmes, et lorsqu'ensuite l'expérience aura donné une conclusion semblable, il ne restera plus de doute et la démonstration sera romplète. ")

1. - A. Jondax : Observations sur plusieurs plantes nouvelles, rares ou critiques de la rrance (3e fragment). Ann. Société Linnéenne de Lyon, $1846, \mathrm{pp}$. 188-189. 
Dans la recherche des caractères distinctifs, les plus saillants ne sont pas les meilleurs. JoRdan découvre que les graines fournissent d'ordinaire un critérium très sûr, et désormais, il l'utilisera dans toutes ses recherches. Ainsi, les graines de $V$. pallescens ont $1^{\mathrm{m}} /{ }^{\mathrm{m}} 1 / 3$ en longueur et deux tiers de millimètre en largeur $; V$. segetalis, $1^{\mathrm{m}} /{ }^{\mathrm{m}} 1 / 2$ de long et deux tiers de large; $V$. agrestis, $1^{\mathrm{m}} /^{\mathrm{m}} 3 / 4$ de long, sept huitièmes de large; $V$. gracilescens surtout a de grosses graines, longues de deux millimètres, larges de un millimètre; il s'agit ici de Viola tricolor annuels, dont le semis est facile et le contrôle parfait. La même règle est valable pour distinguer les Pensées sauvages vivaces, et JoRDAN sépare une Viola sudetica du Mont Forez, d'une autre Viola sudetica du Mont Lozère, parce que les graines de la première ont $1^{\mathrm{m}} /{ }^{\mathrm{m}} 1 / 2$ de long pour $1^{\mathrm{m}} /{ }^{\mathrm{m}} 1 / 5$ de large, tandis que les graines de la seconde ont $2^{\mathrm{m}} / \mathrm{m}$ de long sur $1^{\mathrm{m}} /{ }^{\mathrm{m}}$ de large. En même temps, la séparation des tỵpes en culture met en valeur un grand nombre de caractères stables, jusque-là passés inaperȩus.

Les résultats obtenus par JoRdan furent très discutés. Ils détruisaient la belle ordonnance acquise dans l'exposé des espèces systématiques, qu'il convient maintenant d'appeler espèces linnéennes. Thuret, en Franec, de Bary, NaEgeli, Rosen, en Allemagne, Wiтtrock, en Suède, et bien d'autres depuis, ont retrouvé en des lieux divers les espèces découvertes par JoRDAN et ils ont vérifié le maintien de leurs caractères par des cultures prolongées. On peut done tenir pour rigoureusement établie la proposition que JORDAN énonce dans l'introduction de ses Diagnoses (1864):

"LinNÉ n'admettait au rang d'espèces que les formes qui pouvaient être distinguées au premier coup d'œil et dont le 
signalement était facile à donner. Il en est résulté que la plupart des espèces linnéennes sont plutôt des assemblages de formes spécifiques que des assemblages d'individus; ce sont les premiers groupes qu'on peut établir par le rapprochement des formes similaires et nullement de vraies espèces. ))

Avant de passer à l'examen des conceptions de l'espèce ế de la variété dans les formes cristallines, d'après Pasteur, je veux vous présenter les traits particuliers de la méthode suivie par JORDAN et surtout ses procédés d'induction et de démonstration qui font de ses recherches, je ne dis pas de ses interprétations, l'édifice le plus solide des sciences biologiques.

Jordar s'est donné de l'espèce une définition logique qui est strictement celle de Lrnné ; mais il n'admet pas que le savant préjuge de la valeur relative des caractères différentiels qui, dans chaque cas particulier, doivent être utilisés pour la distinction des espèces. La subordination des caractères est un procédé utile pour la distribution sériée des êtres ; elle facilite leur étude et la recherche de leurs parti. cularités; la subordination permet la synthèse des faits, elle n'a rien à faire avec l'existence même des espèces.

Les espèces sont ou ne sont pas. Il faut examiner les exemples les plus simples pour lesquels le groupement autour de formes-types ne Jaisse aucun doute, noter avec soin et dans le plus menu détail les enchaînements qui permettent d'affirmer après l'examen d'un petit nombre de caractères que tel ou tel assemblage de caractères sera nécessairement accompagné ou suivi d'un certain nombre d'autres caractères. Il faut vérifier surtout qu'il y a répétition de phénomènes semblables par génération sexuée, et plus rigoureuse 
sera la similitude, plus grande sera la certitude que les caractères examinés sont bien le critère de l'espèce. La méthode qui a dominé les recherches de Jordan repose donc sur la fréquence des enchaînements parallèles des phénomènes dans les espèces affines. Elle consiste à mettre en présence deux séries parallèles de faits, l'une bien connue, l'autre à élucider ; en calquant en quelque sorte les enchâ̂nements des termes de la série connue après l'avoir transposée sur quelques termes choisis de l'autre série, on édifie une image de ce qu'il faut constater dans la seconde ; guidé par une induction légitime, on déconvre le fait ou constate l'erreur d'interprétation. L'expérience, qui est ici le semis répété, permet seule de vérifier que l'attribut supposé caractéristique de l'espèce lui appartient en propre, c'est-à-dire se répète sur tous les descendants, ou bien qu'il est accidentel et dû aux conditions particulières de croissance extérieures à l'espèce.

Cette méthode n'est, à mon avis, qu'une application délicate, exigeant du tact et une longue éducation, de la méthode qui permet aux chimistes, en particnlier à Dumas et à Peligot, de préparer, dès la découverte de la nature de l'esprit de bois, ou alcool méthylique (1838), une série de composés parallèles à ceux qu'avait donnés l'alcool commun.

Dans les sciences biologiques, où la variété et la complexité des phénomènes laissent ouvertes tant de directions fausses, il fallait multiplier les séries parallèles, afin d'éviter les nombreux contrôles coûteux et lents, puisqu'ils exigent des semis répétés durant une dizaine de générations. Aussi voyonsnous JoRDAN former des séries de formes de graines, où interviennent la taille et surtout les ornements, des séries de modes de ramification des grappes qui traduisent les inflnences réciproques des bourgeons d'un même axe, des 
séries de divisions et de denticulations des stipules, organes latéraux des feuilles qui se différencient avant elles et sont noins sensibles anx altérations dues au milieu. Lorsque des espèces eritiques présentent, pour trois séries de caractères aussi dirergents, des étapes ou positions d'équilibre en quelque sorte prévues par l'étude des espèces affines dont l'existence est prouvée, il y a de grandes chances de mettre leur existence propre en valeur par quelques semis successifs; ces chances sont mulles ou faibles dans le cas contraire.

Louis PASTEUr assista, presque au début de sa formation intellectuelle, à cette merveilleuse élaboration des découvertes dues à la méthode des parallèles en chinie et, d'instinct, il se perfectionna dans son maniement, d'abord par l'étude des caractères de détail, plus tard par la généralisation des règles dont il vérifiait les conséquences ; il réussit (1S48) à définir avec certitude les relations qui existent entre la forme des molécules cristallines et le pouvoir rotatoire de ces mêmes molécules en dissolution aqueuse ou autre. Gernez (1864) les vérifia pour les corps à l'état gazeux.

M. R. Vallekry-Radot nous représente Louis Pasteur, d'après les lettres conservées par sa famille et par son canna. rade Crinppuis, dans cette période où il fut agrégé préparateur de BaLARD, simple, grave, presque timide, absorbé par l'étude d'une série de corps dérivés de l'acide tartrique. En 1846, il rencontra au Laboratoire un chimiste distingué, Auguste LAURENT, esprit ardent, inquiet, qui venait de préeiser le mécanisme des substitutions, pressenti par Dumas dès 18:34. Dans cette théorie, les espèces chimiques sont, dit 
Pastrur, " comme des édifices moléculaires dans lesquels on pouvait remplacer un élément par un autre sans que l'édifice fût modifié dans sa structure ». Par une application méthodique de procédés qui constituent la formation de tout chimiste, la notion de série des formes affines se précise dans l'esprit de PASTEur. Une autre circonstance, rapportée par II. Vallery-Radot (1), le met en présence des variétés d'un même corps : “ Un jour, il arriva que M. LAUnent étudiant, si je me rappelle bien, un tungstate de soude parfaitement cristallisé... me fit voir an microscope que ce sel en apparence très pur était évidemment un mélange de trois espèces de cristaux distincts, qu'un peu d'habitude des formes eristallines permettait de reconnaître sans peine... Alors, pour acquérir l'habitude des mesures goniométriques, je me mis à étudier avee soin les formes d'une très belle série de combinaisons qui toutes eristallisent avee la plus grande facilité, l'acide tartrique et les tartrates. „) De LE ProvosTAYE venait d'en faire une description détaillée, circonstance heurense " qui me permettait, dit PASTEUR, de comparer à ehaque instant mes observations avec celles, toujours si pré. cises, de cet habile physicien " (2).

Deux années passent; un travail sur les sels de l'acide arsénieux et une étude des phénomènes relatifs à la polarisation rotatoire des liquides lui font décerner le titre de docteur ès-sciences (1847) ; mais la cristallographie forme tou-

1. - La Vie de Pasteur, 1903, p. 38.

2. - Le diplôme d'études exigé actuellement des candidats à l'agrégation doit être un entrainement à la recherche et il serait utile que les élèves consentissent à s'astreindre à la répétition aussí parfaite que possible d'un travail sẻrieux, plutôt que de chercher, sans méthode et sans technique, à mettre en évidence quelque fait nouveau, presque toujours sans intérêt. 
jours l'essentiel de ses recherehes. Le 20 mars 1848, il en donne l'exposé dans un Mémoire présenté à l'Académie des Sciences et intitulé Recherches sur le dimorphisme. C'est un véritable travail de naturaliste, comme le prouve la conclusion : Dans les substances dimorphes, "l'une des deux formes qu'elles présentent est une forme limite, une forme en quelque sorte placée à la séparation de deux systèmes, dont l'un est le système propre de cette forme, et l'autre le système dans lequel rentre la seconde forme de la substance $)$.

Mais la mise en œuvre présente, elle aussi, les traits particuliers de toute recherche de la Systématique. Pasteur a recours à la grande compétence de son maître DeLafosse pour établir une liste, aussi complète que possible, des corps connus pour posséder le dimorphisme (voir p. 37), soit vingt-neuf groupes; il déclare de suite que certains ont des formes cristallines trop peu nettes, ou trop petites, pour pouvoir être déterminées, et il laisse de côté l'examen de six substances « dont l'étude se trouve actuellement neutre quant aux résultats des recherches ».

Ceçi posé, il entre dans le cœur du sujet en comparant entre elles les deux formes cristallines du Soufre (fig. 2), corps simple bien connu, qui ne peut laisser aucun doute sur l'identité de composition chimique des deux variétés; d'ailleurs, l'une se transforme en l'autre sans résidu. Je n'ai pas l'intention de décrire les phénomènes du dimorphisme qui trouvent leur place dans l'étude de la variation (Chap. II) ; j'insiste seulement sur la sériation des faits de même ordre, sur l'analyse parfaite d'un ou de quelques exemples qui ne laissent aucune prise à la critique et sur le procédé de généralisation.

Après l'examen des eas les plus nets, PaSTEur passe à 
l'étude des cristaux des chlorures de naphtaline et de naphtaline monochlorée; les mesures ne sont pas ici assez précises pour que l'on puisse placer une certaine forme dans un système plutôt que dans un autre ; c'est l'exemple même de l'espèce critique telle que la conçoivent les botanistes. PASTEUR est conduit à contredire, quant à la position du cristal étudié, son guide, MI. LaUReNT, pour qui il montre à la même page tant de déférence. Plus loin, à propos de l'acide titanique, il dit : Rien " ne prouve jusqu'ici son isomérie ;
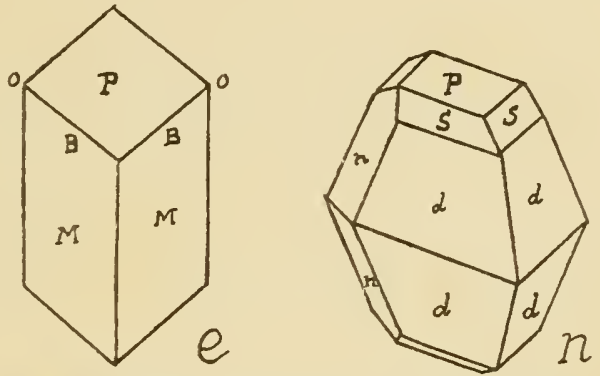

Fig. 2. - Dimorphisme du Soufre cristallisé : $n$, forme normale octaédrique ; $e$, forme exceptionnelle instable prismatique.

je suis seulement porté à l'admettre parce qu'il ne nous ofïre pas les relations que j'ai signalées précédemment. II serait important de constater, par exemple, si chacun de ses états ne correspond pas à une capacité de saturation différente, et s'il n'y aurait pas là un exemple d'isomérie analogue à celui que M. LAURent a fait connaître pour l'acide tungstique. ") Ainsi, certitude dans les premiers cas, déduction logique pour le chlorure de naphtaline que la règle est valable parce que aucun fait précis ne s'y oppose; explication toute différente pour l'acide titanique, en raison de divergences explicables par d'autres considérations. C'est 
le procédé, sécurité de décision et réserves faites pour les cas douteux, que Jordan applique à la même date pour la s.pparation des espèces affines végétales.

La méthode apparaît avec plus de netteté encore dans les études sur la dissymétrie moléculaire des tartrates. Dans le travail précédent, PASTEUR :'est familiarisé en réalité avec la variation, avec le phéinomène que les naturalistes actuels désignent sous les noms d'adaptations doubles, on dimorphisme saisonnier. Chez les tartrates, le dimorphisme est stable; il constitue de véritables éléments distincts dans l'espèce, qui seront désormais pour nous les espèces élémentaires (la désignation varif́té systématique, généralement adoptée, prête à confusion, et il vaut mieux l'éviter).

Il faut iei encore lire le remarquable exposé par Duclaux de l'enchaînement des idées qui conduisit PASTEUr à la découverte essentielle, premier chaînon de la série de ses géniales déductions.

Les cristaux des divers tartrates appartiennent à différents systèmes, ont des formes variées, aiguilles, tables aplaties, prismes ; ils portent des facettes qui abattent leurs angles ou leur's arêtes. Malgré les multiples aspects, le cristallographe réussit à orienter, à identifier les axes vrais. PASTEUr passe en revie les formes cristallines des tartrates neutres et des bitratrates d'ammoniaque, de potasse, de soude, de plusieurs tartrates doubles, des émétiques, des paratartrates et de l'acide tartrique lui-même ; il conclut (1) :

1. - Recherches sur les relations qui peuvent exister entre la forme cristalline, la composition chimique et le sells de la polarisation rotatoire. Annales de Chimie et de Physique, $3^{\mathrm{e}}$ série, t. XXIV (1848), p. 455. 
“ D'une part, tous les tartrates, quelle que soit leur composition chimique, dérivent d'un prisme droit ou très peu oblique, à base rectangle, dont deux dimensions sont sensiblement les mêmes, la troisième variant seule avec la composition élémentaire... ; en second lieu, j'ai montré que tous les tartrates étaient hémiédres ct j’ai signalé ce fait que, dans la plupart, l'hémiédrie avait le même caractère. Elle était accusée par quatre facettes dont le prolongement donnait lieu à un tétraédre, et l'orientation de ce tétraédre, par rapport aux faces principales du cristal, était la même. " L'acide tartrique, les tartrates ordinaires, les tartrates doubles isomorphes de soude et de potasse, de soude et d'ammoniaque, ne présentent jamais l'orientation inverse des faces du tétraédre. Or, toutes les solutions de ces corps dévient dans le même sens, à droite, le plan de polarisation de la lumière.

Mitscherlich, en 184t, fit connaître à l'Académie des Sciences que le paratartrate double de soude et d'ammoniaque est indifférent, alors que le même tartrate double est actif ; or, ces corps ont la même composition chimique, la même forme cristalline avec les mêmes augles, le même poids spécifque, la même double réfraction et, par conséquent, les mêmes angles entre les axes optiques. Dissous dans l'eau, leur réfraction est la même; alors que le tartrate dissous dévie le plan de la lumière polarisée, le paratartrate est indifférent, comme M. Bıот l'a trouvé pour toute la série de ces deux genres de sels; la nature et le nombre des atomes, leur arrangement et leur distance, sont-ils les mêmes dans les deux corps comparés ?

Voilà pour PASTEUR un nouveau cas critique. Si ces corps sont les mêmes, et tout semble l'indiquer, ils devraient posséder le même arrangement moléculaire. Il compare le tar- 
trate neutre d'ammoniaque au paratartrate; il constate qu'ils cristallisent tous deux dans le système du prisme oblique à base rectangle (fig. 3) ; la forme primitive simple ne se rencontre pas, et la modification $b^{2}$, portant sur l'arête $B$, est sensiblement la même dans les deux cas. On a toujours :

Angle $P$ sur $M$, voisin de $90^{\circ}$;

Angle $P$ sur $b^{1}$, voisin de $130^{\circ}$.
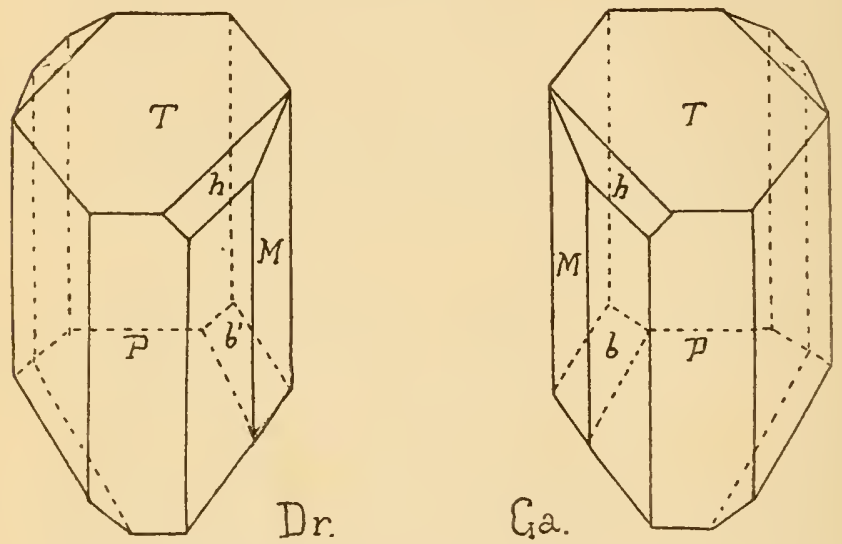

Fig. 3. - Tartrate cristallisé droit $d r$; tartrate gauche ga.

“ Il n'y aura de différent, dans les formes cristallines de tous ces sels, que les facettes placées aux côtés de la forme primitive. ") Ces facettes définissent l'hémiédrie, et, à partir de ce moment, les recherches de Pasteur portent sur ce point spécial :

" Lorsque j'eus découvert l'hémiédrie de tous les tartrates, je me hâtai d'étudier avec soin le paratartrate double de soude et d'ammoniaque; mais je vis que les facettes 
tétraédriques, correspondant à celles des tartrates isomorphes, étaient placées, relativement aux faces principales du cristal, tantôt à droite, tantôt à gauche, sur les différents cristaux que j'avais obtenus. Prolongées respectivement, ces facettes donnaient les deux tétraédres symétriques dont nous parlions précédemment. Je séparai avec soin les cristaux hémiédres à droite, les cristaux hémiédres à gauche ; j'observai séparément leurs dissolutions dans l'appareil de polarisation de M. Вıот, et je vis, avec surprise et bonheur, que les cristaux hémiédres à droite déviaient à droite, que les cristaux hémiédres à gauche déviaient à gauche le plan de polarisation. " PASTEUR s'assure alors, en chassant l'ammoniaque par la chaleur, en présence d'une solution de soude caustique, que cette alternative subsiste intacte pour les deux tartrates de soude obtenus. Il en extrait les acides correspondants et constate la même loi. "En définitive, l'acide paratartrique des chimistes, inactif sur le plan de la lumière polarisée, est composé de deux acides dont les rotations se neutralisent mutuellement, parce que l'un dévie à đroite, l'autre à gauche, et tous deux de la même quantité absolue. »"

Qu'il s'agisse du sel double paratartrate de soude et d'ammoniaque, du sel dérivé paratartrate de soude ou de l'acide paratartrique, la solution est mixte et, daus les trois cas, neutre pour la lumière par neutralisation des deux déviations individuelles égales et de sens opposé.

En 1860, PASTEUr présente ses conclusions à la Société chimique de Paris sous la forme suivante : “ J'avais séparé par la eristallisation du paratartrate double de soude et d'ammoniaque deux groupes atomiques symétriquement isomorphes..., deux espèces de cristaux représentant deux sels distincts, d'où l'on peut extraire deux acides différents... 
" Limn d'eux, celui qui provient des cristaux du sel double hémiédre à droite, dévie à droite, et il est identique avec l'acide tartrique ordinaire. L'autre dévie à gauche comme le sel qui le fournit. La déviation imprimée par ces denx acides aux plans de polarisation est rigoureusement la même en valeur absolue... Si l'on vient à mêler des solutions un peu concentrées de poids égaux de chacun d'eux, leur combinaison s'effectue avec dégagement de chaleur, et la liqueur se solidifie sur-le-champ par une cristallisation abondante d'acide paratartrique, identique à l'acide paratartrique naturel.

"Relativement à leurs propriétés chimiques et cristallographiques, tout ce que l'on fait avec l'un des acides peut se répéter avec l'autre dans les mêmes conditions, et dans tous les cas on obtient des produits identiques, mais non superposables, des produits qui se ressemblent comme la main droite et la main gauche. Mêmes formes, mêmes faces, mêmes angles, hémiédrie dans les deux cas. La seule dissembiance est dans l'inclinaison droite ou gauche des facettes hémiédriques, et dans le sens du pouvoir rotatoire. ) Et Pasteur adopte la définition de l'espèce de Chevreul, que nous ferons nôtre :

" L'espèce chimique est la collection de tous les individus identiques par la nature, la proportion et l'arrangement des éléments (1823). ») Dans les corps isomères, tels que les acides tartriques, la nature et les proportions sont les mêmes; l'arrangement seul diffère : des corps peuvent donc être et sont spécifiquement différents par cela seul que l'arrangement des atomes n'est pas le même dans leurs molécuies chimiques. 


$$
+\%
$$

Les objets matériels examinés sous le rapport de leurs formes et de la répétition de leurs parties identiques se distribuent en deux classes : les uns, placés devant une glace, doment une image qui leur est superposable, cube, tige à feuilles opposées du Lilas; l'image des autres, tétraèdre irrégulier, tige à feuilles insérées en spirale du Rosier, ne pourrait les recouvrir, bien qu'elle reproduise fidèlement tous leurs détails ; ces derniers n'ont pas de plan de symétrie. L'acide tartrique ordinaire droit et l'acide tartrique gauche, préparés comme il vient d'être dit, appartiennent à la seconde classe.

Remontant à l'essence des choses, il faut que la molécule de l'acide tartrique soit dissy'métrique et d'une dissymétrie à image non superposable. De même, toutes les combinaisons chimiques se partagent en corps à image superposable et corps à image non superposable.

$\mathrm{Or}$, " tous les produits artificiels des laboratoires et toutes les espèces minérales sont à image superposable. Au contraire, la plupart des produits organiques naturels, tous les produits essentiels de la vie sont dissrmétriques et de cette dissymétrie qui fait que leur image ne peut leur être superposée $n$.

Le quartz, produit naturel, est formé de cubes ; fondu, il n'agit pas sur la lumière; c'est l'arrangement particulier des cubes dans le quartz cristallisé qui modifie la lumière. Il n'est done pas moléculairement dissymétrique. Il n'y a qu'une seule espèce de quartz.

Les chimistes préparent à partir du camphre naturel, qui est dissymétrique, l'acide camphorique également dissymé- 
trique, à partir de l'asparagine, l'acide aspartique dissymétrique à la manière de l'asparagine ; il n'est pas douteux que l'origine de la dissymétrie doit être reportée au produit naturel qui conserve dans les réactions accessoires sa charpente propre. Mais les substances artificielles proprement dites, l'alcool par exemple, n'est pas dissymétrique, et pas un dérivé de l'alcool ne l'est.

Il y a plus : soumis à des réactions chimiques énergiques, les groupes naturels perdent leur propriété constitutionnelle; l'acide tartrique est dissymétrique, l'acide pyrotartrique ne l'est plus ; l'acide malique est dissymétrique, les acides maleïque, paramaleïque, ne le sont plus; la gomme est dissymétrique, l'acide mucique ne l'est pas...

"Cellulose, fécules, gommes, sucres..., acides tartrique, malique, quinique, tannique..., morphine, codéine, quinine, strychnine, brucine..., essences de térébenthine, de citron..., albumine, fibrine, gélatine..., tous ces principes immédiats sont dissymétriques; toutes ces matières ont le pouvoir rotatoire à l'état de dissolution ; caractère nécessaire et suffisant pour établir la dissymétrie (moléculaire). »

Il $\mathrm{y}$ a beaucoup de substances naturelles qui ne le sont pas : l'acide oxalique et l'acide fumarique des végétaux, l'urée, la créatine, le glycocolle dans l'organisme animal. Ne fant-il pas y voir des dérivés des substances naturelles proprement dites ? des excrétions plutôt que des sécrétions ? I] se peut aussi que la dissymétrie soit au fond, mais cachée, comme dans les paratartrates, par la neutralisation de deux dissymétries inverses.

Ces déconvertes sont le premier pas de Pasteur dans le domaine de la vie. Nous allons constater qu'il s'y engage avec la prudence et la technique du chimiste le plus avisé. 
Un collègue, professeur à Vendôme, Dessaignes, annonce avoir transformé par la chaleur les fumarate et maléate d'ammoniaque en acide aspartique. Le dernier est dissymétrique, les premiers ne l'étant pas. PASTEUR s'assure que l'acide aspartique de Dessaignes n'est qu'un isomère de l'acide aspartique dérivé de l'asparagine naturelle ; il en possède toutes les propriétés, sauf la propriété rotatoire de ses sclutions. Il y a done un acide aspartique actif, et un antre inactif.

La différence se maintint dans les transformations des deux acides aspartiques en acides maliques, qui ne diffèrent de même que par leur action sur la lumière polarisée. Or, à ces compositions chimiques et propriétés identiques se superposent des arrangements moléculaires différents; le bimalate, dérivé du produit de DeSSAIGNES, diffère du bimalate dérivé de l'asparagine par l'absence de quatre petites faces hémiédriques sur les formes cristallines. "Qui pourrait douter, d'après cela, dit PASTEUR, des rapports d'arrangements moléculaires de ces deux sels ? N'est-il pas évident que nous avons ici affaire à un acide malique identique au naturel, sauf la simple suppression de la dissymétrie moléculaire ? )

Il y a done des corps inactifs et symétriques qui correspondent aux corps de même composition, mais à molécules dissynétriques, ce qui fournit la notion de petite espèce dans une même espèce chimique. Mais parce qu'il connaît l'essence même de la différence, PASTEUr va plus loin, il devine: " Chaque substance dissymétrique offre quatre variétés, ou mieux, quatre sous-espèces distinctes : le corps droit, le corps gauche, la combinaison du droit et du gauche et le corps qui n'est ni droit ni gauche, ni formé par la combinaison du droit et du gauche. » 
Avec une intuition de génie, qui me paraît trouver des justifications définitives dans la production expérimentale de petites espèces vivantes nouvelles par mutation (p. 76), Pasteur déclare : "Je regarde comme nécessaire la conclusion de l'existence de forces dissymétriques au moment de l'élaboration des produits organiques naturels, forces qui seraient absentes ou sans effet dans les réactions de nos laboratoires, soit à cause de la brusque action de ces phénomènes, soit pour toute autre circonstance incomnue. " La durée des réactions dans un milieu défini caractérise mieux que tout autre critérium les divergences d'équilibre des espèces affines, mieux même que les modifications de formes si variées des organes d'un même individu pluricellulaire ; l'identité des réactions des divers individus d'une même espèce est un autre critérium de l'unité spécifique. Nous l'étudierons dans le chapitre suivant. 


\section{CHAPITRE II}

\section{LA VARIATION DES FORMES}

Le bel enchaînement des travaux de cristallographie réalisés successivement pas Louis PASTEUR ne pent être le fait du hasard; il est incontestablement la marque du génie qui passe en revue, dans un ordre naturel et logique, les phases distinctes et essentielles d'une suite de phénomènes. Après avoir acquis une notion expérimentale de l'espèce et des sous-espèces, PAsteur étudie la variation des formes et leur dépendance des circonstances de formation, puis les altérations dues à la combinaison de corps possédant chacun une dissymétrie propre, enfin les phénomènes de régénération et les règles de la croissance. Des tâtonnements d'vn demisiècle ont conduit les naturalistes modernes à adopter une distribution identique des phénomènes de la vie ; ayant défini les espèces élémentaires par l'épreuve des semis répétés, ils étudient la variation de ces espèces sous l'influence du milieu, les combinaisons hybrides, les formes ataviques et l'accélération embryogénique; l'ensemble met en valeur les lois générales du polymorphisme.

Dès 1850, Pasteur possède un critérium précieux qui lui sert désormais de guide; l'hémiédrie n'est qu'un aspect fréquent de la dissymétrie moléculaire ; la déviation du plan de polarisation par les solutions des substances dissymétriques est le caractère essentiel, constant. A cause de sa 
généralité, la subordination du premier caractère au second s'impose. Désormais seront appelées dissymétriques les substances définies dont les solutions dévieront, peu ou beaucoup, le plan de polarisation.

Cette conception apparaît clairement dans la première partie d'un remarquable Mémoire (1), paru en 1853, einq années après la découverte du principe. Le titre, Production forcée de l'hémiédrie non superposable dans les substances actives sur la lumière polarisée qui n'offrent pas habituellement ce caractère, met en relief la préoccupation du savant qui veut généraliser sa découverte :

" Dans la pluralité des cas, les formes cristallines des substances actives sur la lumière polarisée possèdent l'hémiédrie non superposable. Cependant, j'ai rencontré un certain nombre de substances actives qui se présentent toujours avec des formes cristallines homoédriques. La corrélation des deux phénomènes souffre-t-elle done des exceptions ? "

N'est-ce pas un accident provoqué par les conditions de cristallisation? Certains tartrates, dans les circonstances ordinaires, ne portent pas les facettes hémiédriques, et, dans ce cas, il y a identité parfaite et absolue entre les formes cristallines des deux sous-espèces, déviant l'une à droite, l'autre à gauche, le plan de polarisation.

" Une espèce minérale, qui a manifestement et toujours une structure cristalline hémiédrique, est bien loin de l'aceuser constamment par la symétrie de ses modifications... J'ai pensé qu'il suffirait de modifier les circonstances de sa cristallisation pour faire apparaître forcément, et d'une manière constante, les facettes hémiédriques. "

1. - Nouvelles recherches sur les Relations... Annales de Chimie et de Physique, $3^{\mathrm{e}}$ série, t. XXXVIII, p. 437. 
Le bimalate de chaux, plus soluble à chaud qu'à froid, donne facilement naissance à des cristaux volumineux ; lorsqu'il se forme dans l'eau pure, il n'est jamais hémiédrique ; l'hémiédrie est cependant un caractère de la molécule de l'acide malique, et la combinaison simple avec la chaux n'a pu altérer ce caractère fondamental ; d'ailleurs, la solution du bimalate de chaux ordinaire dévie le plan de polarisation. En conséquence, il doit y avoir des circonstances où l'hémiédrie cristalline se manifestera; en provoquant la cristallisation dans l'acide nitrique, PASTEUR fait apparaître l'hémiédrie sur tous les cristaux (fig. 4).

Cette confirmation de la logique du raisonnement est d'autant plus intéressante que, pour cet excmple même, il existe deux bimalates de chaux inactifs renfermant des quantités d'eau de cristallisation différentes et qui peuvent se former tous deux soit dans l'ean pure, soit dans l'acide nitrique faible. L'un d'eux possède exactement la même composition, la même forme cristalline que le bimalate actif, mais ne donne jamais les facettes hémiédriques, quel que soit le mode de cristalli-

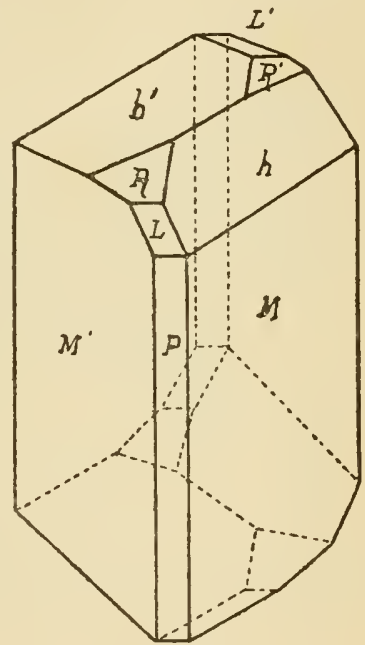

Fig 4. - Cristal de bimalate de chaux obtenu par dépôt dans l'acide nitrique (d'après Pasteur). sation employé. Il se comporte comme l'une des quatre sous-espèces de l'acide tartrique dont PASTEur a prévu l'existence ; il diffère fondamentalement du bimalate actif. Dans le cas où l'on possède le bimalate actif, l'addition 
d'acide nitrique à la solution aqueuse fait apparaître les faces hémièdres. Dans les solutions faibles, les cristaux ont la forme de la figure 4 ; pour une certaine concentration de l'acide, les cristanx, encore hémiédriques, présentent un tout autre aspect.

Dans la classification des végétaux, les botanistes ont rencontré des phénomènes analogues ; souvent de longues comparaisons et toujours des épreuves expérimentales furent nécessaires pour démontrer l'identité spécifique des formes accommodées à différents milieux. M. DE VRIEs (1) en a décrit plusieurs exemples sous le titre Adaptations doubles, précisément pour montrer que l'espèce n'est pas altérée. L'un des plus frappants fut éclairci par les études de M. MASSART, professeur de botanique à l'Université de Bruxelles (1902).

Les méthodistes décrivent depuis longtemps deux formes de Renouée, Polygonum natans Mœnch (fig. 5, n), Polygonum terrestre Leers (fig. $5, t$ ), dans l'espèce linnéenne Polygonum amphibium; GRENIE' et GoDron, dans leur flore française, KосH, dans sa flore allemande, les distinguent comme des sous-espèces. Mr. Massart a montré que la transplantation de fragments d'une même tige dans des milieux différents provoque l'apparition à volonté de l'une ou de l'autre forme.

Lorsque des plantes flottantes, à tiges longues et lisses, terminées par une touffe de feuilles glabres et luisantes, ovales et longuement pétiolées (fig. $5, n$ ), sont amenées sur la rive, elles y développent uniquement des tiges raides, dressées, presque dépourvues de ramifications, por-

1. - Espèces et variétés, leur naissance par mutation, trad. BLARinghes, Paris, F. Alcan, 1909, p. 271. 
fragments de plantes de la forêt de Fontainebleau en haute montagne et, inversement, de la montagne dans la plaine, rentrent dans cette catégorie de phénomènes.

La systématique des Insectes, surtont celle des Papillons, est encombrée d'une profusion de soi-disant espèces locales qui n'ont pas d'autre valeur. Les célèbres expériences de Standfuss (1898) en donnent la preuve ; elles ont duré douze années et porté sur plus de 42.000 chenilles.

Il y a lieu de distinguer avec StandFuss les températures qui n'altèrent pas sensiblement l'organisme et modifient simultanément toutes les chenilles, à savoir le chaud (37 à $39^{\circ}$ ) agissant durant deux ou trois jours et le froid $\left(4\right.$ à $\left.6^{\circ}\right)$ agissant durant quatre à huit semaines, des températures extrêmes $\left(40\right.$ à $\left.45^{\circ}\right)$ et $\left(0^{\circ} \grave{a}-18^{\circ}\right)$ qui tuent la majorité des organismes et altèrent réellement les quelques êtres résistants.

Par des épreuves ménagées du chaud et du froid sur les chrysalides Vanessa C. Album, Polyommatus Amphidamas, Standfuss obtient soit la forme d'été, soit la forme d'automne. Par le froid, Vanessa urticae de Zurich est transformée en la variation polaris de Laponie, par le chaud, en la variation ichnusa de Corse; par le chaud encore, les chrysalides d'antomne de Papilio polidarius évolnent pour la plupart en papillons de la génération d'été et, pour quelques cas, en la variété Zanacleus de Sicile.

Il se présente aussi des modifications de l'ordre des caractères sexuels secondaires ; par le chaud, Parnassius Apollo L. femelle prit l'aspect mâle (fig. 6) ; par le froid, Rhabdocera Rhamni femelle acquit des ailes d'un jaune intense, et STANDFuss fait remarquer que chez certaines la modification semble liée à des changements dans les glandes embryonnaires. 
Des formes très rares ou inconnues de Vanessa antiopa furent obtenues au cours de ces expériences, qui sont une
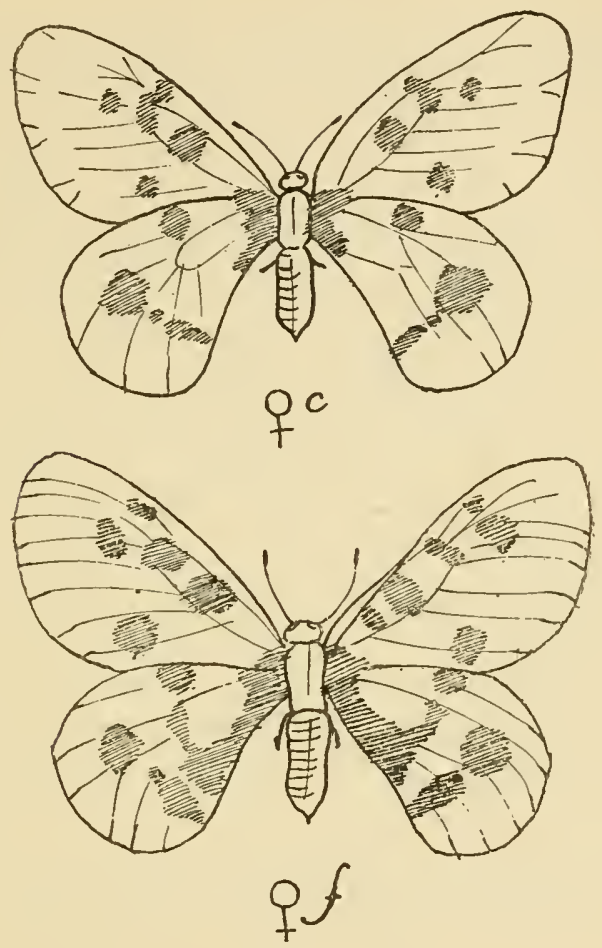

Fig. 6. - Modifications produites par l'action du froid, $t$; par l'action du chaud, $c$; chez la femelle de Parnassius Apollo (d'après STandFuss, 1898).

indication précieuse sur la nature des formes sériées et localisées, si fréquentes chez les papillons. Les remarquables Etudes de Lépidoptérologie comparée de M. Chardes OberTHUR (1906-1922) obligent le lecteur à trouver dans ces 
manifestations un caractère presque général dans I'ordre des Papillons.

Il existe parfois des individus mixtes ayant subi dans un phase de leur vie certaines influences, plus tard des influences différentes; ils associent sur le même être des caractères distincts. Ainsi, d'un même pied de Lierre on sait, par bouturages répétés à l'ombre ou sur les terres humides, multiplier presque indéfiniment les tiges rampantes ou grimpantes à feuilles losangiques, régulièrement distribuées à droite et à ganche des tiges sur la face opposée à celle où se développent les racines adventives crampons. Mais on peut aussi obtenir, de la même plante, un nombre indéfini de petits arbres en boule, en ayant soin de prélever les boutures sur les axes dressés du même pied de Lierre sur le point de fleurir ; ici, plus de racines crampons, plus d'orientation dorsiventrale des rameaux, mais des touffes de branches en buisson, portant uniquement des feuilles allongées, ovales, distribuées comme celles du Poirier. En examinant avec attention la base de ces boutures en arbres, sous les touffes épaisses où la Iumière pénètre mal, on découvre toujours quelques pousses filiformes qui ne peuvent s'étaler sans être soutenues par un support et qui rappellent, avec tous leurs traits, les Lierres grimpants. Il y a done des termes de passage, et, pour cette raison, la dénomination horticole Hedera helix arborea n'a pas été adoptée par les botanistes classificateurs qui notent la variation, mais n'admettent pas sa permanence indépendamment des conditions de vie.

Pasteur étudia des formes de passage analogues dans la cristallisation du bimalate d'ammoniaque. Préparé avec l'acide malique ordinaire extrait des pommes, des raisins et crista!lisé dans l'eau pure, ce composé chimique ne montre 
j๕mais les facettes hémiédriques ; elles apparaissent sur les cristaux dont l'acide malique provient de l'asparagine. Pour les obtenir à volonté et à partir de n'importe quel acide malique, PASTEUr sature cet acide par l'ammoniaque en excès, puis il évapore la liqueur jusqu'à obtenir une teinte brune par l'effet d'un début de décomposition; la masse

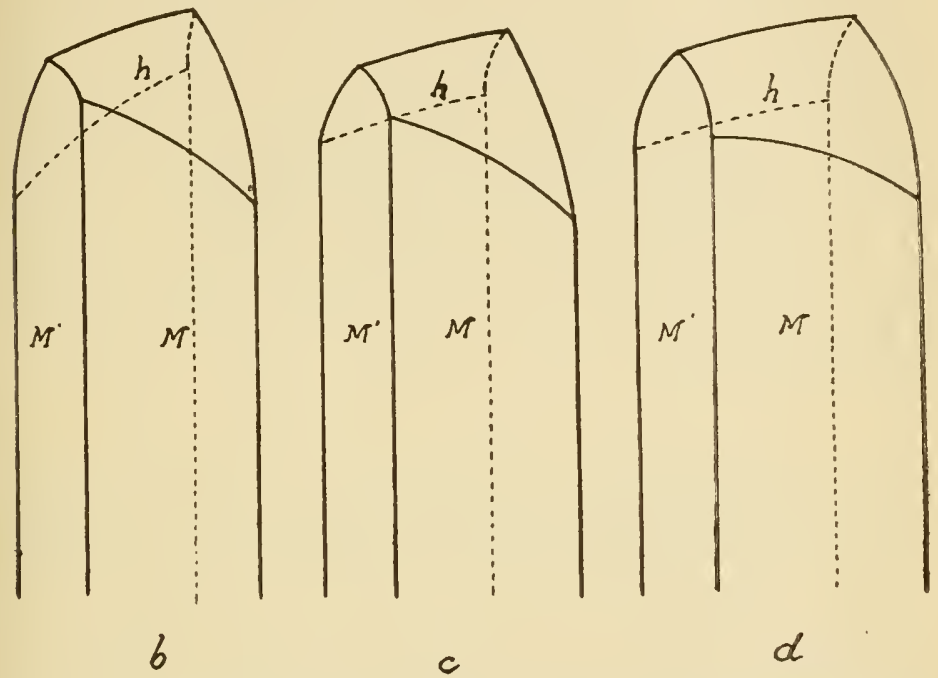

Fig. 7. - Formes à angles arrondis de Cristaux de bimalate dammoniaque obtenues dans les premières cristallisations (d'après Pasteur).

fondue, refroidie, reprise par l'eau, est mise à cristalliser ; elle donne une masse grenue, mamelonnée, souillée par une eall-mère visqueuse, colorée, qu'il enlève en comprimant les cristaux entre des doubles de papier. Reprise par l'eau et mise à cristalliser de nouveau, elle ne foumit que des cristaux hémiédriques. "Une autre cristallisation les domne purs, très limpides et encore tous hémiédriques. Mais, par de 
nouvelles cristallisations, on rend le bimalate parfaitement homoèdre, ce qui prouve que très probablement les eristaux ne sont hémiédriques qu'autant que l'eau-mère, au sein de laquelle ils prennent naissance, renferme quelque produit étranger. ")

La forme cristalline, les angles des faces du bimalate d'ammoniaque, ne changent pas au cours de ces manipulations ; mais les faces hémiédriques $h$ se modifient (1). Dans les premières cristallisations, elles existent seules et font disparaître, par leur développement, les faces $L$ et $R$. De plus, elles sont souvent très courbes (fig. $7, b, c, d$ ) ; ce n'est qu'après la troisième ou quatrième cristallisation depuis qu'il a été chanffé que le bimalate d'ammoniaque donne des cristaux nets, limpides, qui ont la forme de la figure 4 (page 29).

$$
* * *
$$

L'étude du bitartrate d'ammoniaque, du tartrate neutre de potasse et du tartrate double de potasse et d'ammoniaque confirme les faits déjà exposés en faisant apparaître une nouvelle complication qu'entraînent les formes limites. Les naturalistes désignent les formes correspondantes sous le nom d'espèces critiques. Il eût été logique de les traiter dans le chapitre relatif à la notion d'espèce, mais, dans les

1. - Au cours de cet exposé, que Pasteur développe, car il s'agit d'un cas critique, il dèclare : "Ce qui prouve encore que le caractère hémiédrique n'est pas dù à quelque modification éprou. vée par le sel quand on le chauffe, c'est que, si l'on ajoute du bimalate d'ammoniaque ordinaire non hémiédrique à du bimalate qui a été chauffé, on rend hémiédrique le bimalate ajouté. "C'est la première fois dans les œuvres de Pasteur que se présente la notion de germe (ou impuretè) altérant la forme, notion sur laquelle repose ma théorie de l'hérédité mendélienne (p. 61). 
sciences descriptives, l'instabilité des espèces critiques suggère inévitablement l'idée d'une cause prochaine de variation. Pour bien montrer que cette suggestion n'a, le plus souvent, rien de fondé, et pour profiter des enseignements de Pasteur, je vais, avec lui, m'efforcer de distinguer ce qui est variation due au milieu de ce qui est, pour DE VRIEs et moi-même, variété instable.

En réalité, le mémoire de PASTEUR auquel je fais allusion est antérieur à ceux dont j'ai parlé jusqu'ici ; il y décrit des faits d'un ordre tout différent de l'hémiédrie et le titre même pourrait suggérer l'image des adaptations doubles dont il vient d'être question. J'ai done dû préciser la notion d'adaptations doubles avant d'exposer les Recherches sur le dimorphisme (1848).

Pasteur appelle dimorphes " des substances de même composition chimique qui peuvent avoir des formes cristallines différentes et incompatibles )( 1 ) et il en donne une liste en signalant l'aide reçue de son maître DeLAfosse pour l'établir. Ce sont des corps simples : le soufre, le carbone, l'étain ; ou des acides, titanique, arsénieux, antimonieux ; surtout des sels, nitrates de potasse et de soude; des corps naturels, parfois désignés dans la nomenclature ordinaire, sous des noms différents, tels le Grenat, qui appartient au système cubique, et l'Idocrase, de même composition chimique, qui appartient au système du prisme droit à base carrée ; au total, une trentaine d'exemples, la plupart bien déterminés.

Dans le préambule aussi, PASTEur détruit ane erreur courante : " Pour beaucoup de savants, le mot dimorphisme

1. - Annales de Chimie et de Physique, $3^{\text {e }}$ série, t. XXIII (1848), pp. 273-294 (avec 12 fig.). 
veut dire qu'une substance, tout en offrant la même forme cristalline et le même arrangement moléculaire, ou, pour abréger, une même substance, peut offrir deux formes cristallines différentes... ; cela est vraiment inconcevable, à moins de supposer que des molécules d'une forme peuvent s'associer de manière à donner un groupe d'rune autre forme, jouant à son tour le rôle de moléeule intégrante. " L'hypothèse du transformisme spécifique ne le retient pas un instant; il montre, en effet, que " si les arrangements moléculaires ne sont pas les mêmes dans deux variétés (1) dimorphes, il y a entre eux une étroite relation. La différence est assez grande pour provoquer l'incompatibilité de leurs systèmes cristallins : pourtant elle n'a rien de profond. Elle altère les propriétés physiques; elle laisse à peu près les mêmes les propriétés chimiques ».

Voici l'ordre de grandeur des altérations : le soufre cris tallise en prismes rectangulaires droits, dont les pans font entre eux un angle de $90^{\circ}$ et en prismes obliques dont l'angle des pans est $90^{\circ} 32^{\prime}$; l'angle de la base sur les pans est dans le premier cas $90^{\circ}$, dans le second $94^{\circ} 6^{\prime}$ (fig. 2, p. 17). La forme des cristaux naturels, et, en général, celle des cristaux obtenus par évaporation d'une solution dans le sulfure de carbone est du système rectangulaire droit ; la forme obtenue après surfusion, du système du prisme oblique à base rhombe. "Nous dirons, avec Pasteur, que le sonfre, sous ses deux états, correspond à deux équilibres moléculaires très voisins l'un de l'autre, quoique appartenant à deux systèmes différents. L'un de ees équilibres est beaucoup plus stable que l'autre, comme nous allons le voir. Chacun sait que le

1. - Le mot "variété " n'est pas en italiques dans le texte de Pasteur. 
soufre en prisme oblique devient peu à pen opaque, et se trouve alors transformé en soufre octaédrique. Dans certains cas même, il suffit de toucher une aiguille prismatique oblique pour qu'elle devienne subitement opaque » (1).

Après avoir posé la question et esquissé la solution, PASTEur examine les exemples et, de suite, à propos du soufre même, il indique que la clarté des phénomènes présentés par ce corps simple, qu'on peut obtenir avec la plus grande facilité à l 'état de pureté, " ne permet plus à quelques esprits de douter encore de l'identité de composition chimique dans les su'bstances dimorphes "); il montre qu'en choisissant correctement les axes d'orientation des cristaux différents, on peut toujours ramener les deux formes à être très voisines. Autrement dit, il remanie la classification, qui n'est qu'un moyen d'exposer les faits, en choisissant celle qui met le plus clairement en valeur les affinités et il applique, sans s'en douter, le principe et le procédé expérimental qui ont conduit Bernard de Jussieu à la Méthode naturelle.

Le nitrate de potasse et le nitrate de soude sont des substances dimorphes; de plus, ces deux composés de l'acide nitrique sont isodimorphes, e'est-à-dire que les deux formes de chacun de ces corps appartiennent aux mêmes systèmes. " Un des systèmes est le prisme rhomboïdal droit, dont l'angle des pans est très voisin de 120 degrés; l'autre est le prisme hexagonal régulier. Seulement, la forme ordinaire la plus stable du nitrate de potasse est le prisme rhomboïdal, tandis que la forme ordinaire la plus stable du nitrate de soude est le rhomboèdre de $106^{\circ} 30^{\prime}$. La forme habituelle du

1. - Annales de Chimie et de Physique, 3e série, t. XXIII (1848), p. 273. 
nitrate de potasse est un prisme à six pans presque régulier, et souvent les sommets sont des pyramides à six faces très voisines également de pyranides régulières à triangles iso. cèles. Nous retrouvons donc encore ici le dimorphisme là où i! y a forme limite. " Mais Pasteur ne laisse passer aucune occasion de pénétrer plus profondément dans l'analyse du phénomène; des observations de M. Frankenheim lui fournissent la généralisation de la règle de l'équilibre instable énoncée à propos du soufre. "Lorsqu'on fait évaporer une goutte de solution de salpêtre (nitrate de potasse) placée sur une lame de verre, les petits cristaux formés au centre de la goutte sont du système rhomboïdal, et ceux qui se forment sur les bords de la goutte sont du système hexagonal. Il en est de même pour l'azotate de soude. ...L'équilibre moléculaire... instable... rentre aussitôt dans l'équilibre stable qui l'avoisine dès qu'il est dérangé, même par de faibles influences. Ce sont des faits analogues à ceux offerts par le soufre, par l'iodure de mercure, par l'arragonite portée à la température de 100 degrés, par le séléniate de nickel exposé aux rayons solaires, etc... ")

Le sulfate de potasse nous servira de troisième exemple; il cristallise parfois en prisme hexagonal régulier, mais la forme la plus habituelle dérive d'un prisme rhomboïdal droit tr'ès voisin de $120^{\circ}$, et les deux prismes offrent des facettes également inclinées (123 ou $\left.124^{\circ} 20^{\prime}\right)$ sur leurs bases; le le goniomètre accuse la relation des dimensions moléculaires des formes primitives.

M. IIUGo DE VRIES (1902, puis 1905) a longuement insisté sur les phénomènes particulier's présentés au cours de leur croissance par les races instables. La moindre altération dans les conditions de vie fait qu'un organe, tel que la feuille du 
Trèfle, porte trois folioles, ou quatre on cinq folioles ; certaines lignées de Trèfles sont beancoup plus sensibles que d'autres à ces agents externes, tel le Trifolium pratense quinquefolium.

Le Pavot polycéphale fut plus étudié. Il présente cette particularité de fournir constamment, dans certaines conditions de culture, des capsules entourées de couronnes d'étamines métamorphosées en carpelles soudés ou libres (fig. 8). Lorsque les semis sont très clairsemés et le terrain suffi-
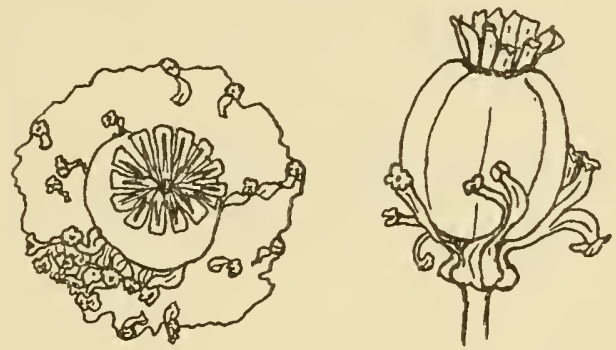

Fıg. 8. - Pavot Polycéphale (Papaver somniferum polycephalum), à gauche, capsule à métamorphoses multiples vue d'en haut; à droite, capsule à métamorphoses rares vue de face (d'après H. DE VRies).

samment riche, presque toutes les étamines sont transformées en carpelles ; lorsque les semis sont serrés, la trace des métamorphoses est tellement atténuée qu'on ne peut souvent distinguer le Pavot polycéphale de la vulgaire Eillette. Jamais l'Eillette, quelles que soient les conditions de semis ou de fumure, ne donne un exemple de la métamorphose d'étamine en carpelle ; la lignée polycéphale reproduit toujours la métamorphose lorsqu'on sème les graines, même provenant de plantes dépourvues de carpelles accessoires durant plusieurs générations successives, dans les conditions d'espace, de nutrition et d'éclairement favorables à l'épa- 
nouissement de l'anomalie. Cette lignée possède donc deux formes d'équilibre qui passent de l'une à l'autre sans qu'il y ait la moindre altération dans l'hérédité, c'est-à-ảire dans la constitution fondamentale moléculaire.

Les formes sexuelles me paraissent rentrer dans la catégorie des phénomènes de dimorphisme étudiés par Pasteur. Sans doute, il serait dangereux de généraliser les quelques exemples dont j'ai pu faire l'étude, guidé par les notions que je viens de rappeler. Je suis convaincu cependant que chez les Lins, pour ce qui concerne la dichogamie, c'est-à-dire la succession de la maturation des pollens et des ovules, que chez les Eillets, les Lychnides et beaucoup d'autres Caryophyllées, que chez les Epinards et les Mercuriales, le pliénomène répond aux conditions établies avec tant de rigueur pal Pasteur pour ce qui concerne le dimorphisme de certaines espèces chimiques. Je renvoie le lecteur à mes Etudes sur le polymorphisme floral s'il désire acquérir quelques notions techniques sur le sujet (1). Et lorsque je vois certains (Fillets (Dianthus barbatus L.) n'offrir que des fleurs blanches dans la phase mâle, que des fleurs rouges dans la phase femelle (2), je suis induit à penser qu'une très légère modification chimique des contenus cellulaires traduit ici d'une manière visible une succession d'étapes, qui peuvent d'ailleurs se stabiliser durant quelques générations dans les colloïdes, oì les réactions sont d'une durée infiniment lente par rapport aux stabilisations des composés minéraux.

Dans le niême Mémoire sur le dimorphisme et dans une

1. - Bulletins de Ia Société botanique de France et C. $R$. Société de Biologie, 1919, 1920, 1921 et 1922.

2. - Couleur et sexe des fleurs : C. R. Soc. Biologie, 12 juin 1920. 
Note relative à un travail de M. LAURENT : Sur l'isomorphisme et sur les types cristallins, PASTEUR fournit l'explication d'une difficulté que le premier savant avait rencontrée dans l'étude des cristallisations de mélanges de chlorures de naphtaline et de naphtaline monochlorée. Les cristaux de ces deux corps, formés dans l'éther à 100 degrés, ont le premier, une forme ramenée au prisme oblique à base rhombe, l'autre, une forme ramenée au prisme droit à base rhombe. LAURENT, après avoir étudié ces deux formes, en avait déduit que des substances différentes pouvaient être isomorphes, quoique appartenant à des systèmes différents; les mélanges de ces chlorures peuvent cristalliser en toutes proportions, sans que la forme eristalline change d'iune manière sensible. PASTEUR trouve cette conclusion incorrecte.

" A priori, on pourrait affirmer qu'une telle proposition est impossible si elle devait impliquer cette autre, que des molécules appartenant à deux systèmes différents, quoique très voisines, peuvent s'associer, se juxtaposer les unes aux autres. Des molécules qui auraient la forme d'un prisme rectangulaire oblique ne pourraient pas, quelque faible que soit l'obliquité, ne fût-elle que d'une fraction de minute, se juxtaposer à des molécules en prisme rectangulaire droit; car une condition essentielle de la cristallisation, e'est que l'espace soit rempli, c'est qu'il n'y ait pas entre les molécules un vide de dimensions finies."

Cet axiome établi, Pasteur montre que l'une et l'autre espèce sont dimorphes, paree que, en orientant convenablement certaines faces, on trouve des variations d'angles de quelques degrés dans chaque espèce; de plus, elles sont isodimorphes. Lorsqu'on les mêle et les fait cristalliser ensemble, " des molécules de même forme et exactement de même angle sont ou peuvent être en présence ». 
J'ai cité cet exemple particulier par analogie avec les chimères végétales de WinkLer (1907), qui associe par la greffe la vulgaire Morelle (Solanum nigrum) et la Tomate (Solanum Lycopersicum) ; il en obtient des fragments d'individus, qu'on sait bouturer et qui peuvent vivre quelques années dans cet état d'équilibre instable, pourvus les uns d'une charpente de Tomate avec épiderme de Morelle, ou inversement, les antres de ramifications offrant des associations plus ou moins complexes de caractères en mosaïque. Il y a, dans ces phénomènes, une curieuse et exceptionnelle coadaptation de tissus qui gardent, presque toujours, leur individualité, mais supportent la coordination, avec échanges à la façon des jumeaux soudés ou frères siamois, ou mieux à la façon de certains parasites avec leur hôte (Gui et Pommier). Ces faits sont dominés à la fois par les règles de la régénération et de la croissance coordonnées des tissus et par la tendance constante à la ségrégation que présente l'association en mosaïque d'espèces distinctes.

$$
\begin{aligned}
& * \\
& * *
\end{aligned}
$$

Les mosaïques végétatives obtenues par WINKLer (1) sont une application de la loi, générale, de la disposition symétrique des tissus et des organes chez les êtres vivants. En fait, les deux masses spécifiques sont d'abord soudées par la greffe (fig. 9) ; puis, lorsque la greffe est prise, l'opérateur supprime le greffon en ayant soin d'entamer le bourrelet dans la zone d'intime contact des éléments d'origines diffé-

1. - Winkler : Ueber die Solanum Profpbastarde (1908). 
rentes. Sur la face de section se développent des bourgeons adventifs constitués par l'assemblage de cellules peu différeneiées; la plupart des bourgeons adventifs reproduisent
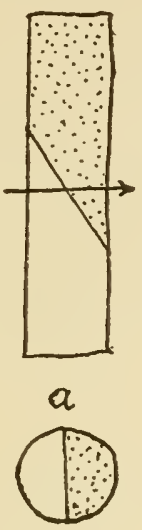
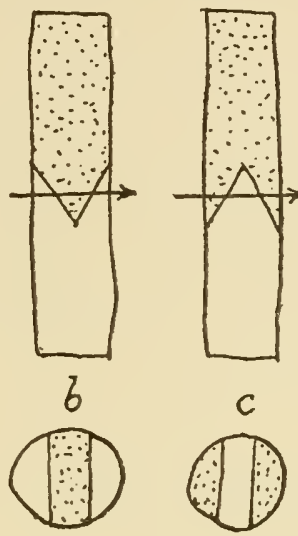

C

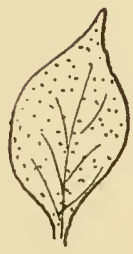

$n$

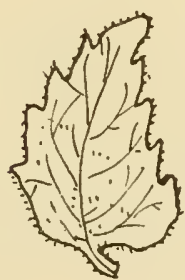

tru
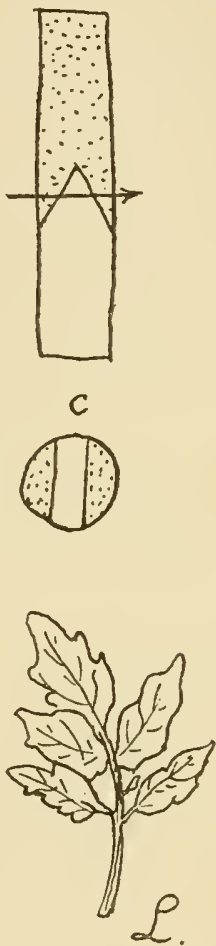

Fig. 9. - Modes de greffe de la Tomate sur la Morelle et sections correspondantes, $a, b, c ; n$, feuille de Solanum nigrum, $t u$, de $S$. tubingense, $L$, de Lycopersicum (d'après WinkLER).

l'une ou l'autre espèce, les cellules plus nombreuses de l'une ou de l'autre espèce éliminant par leur rapide croissance les rares cellules de l'espèce opposée qui ont pu être englobées dans la masse presque homogène. Mais il arrive, rarement, 
que des bourgeons adventifs. sont composés de masses comparables de cellules, appartemant soit au greffon, soit au sujet; la plupart n'évoluent pas à cause de la discordance des tendances divergentes de l'assemblage ; quelques-uns, et plus spécialement ceux dont les cellules de l'une et de l'autre espèce peuvent s'ajuster en formant des tissus (ou feuillets) distincts subsistent. Ainsi Solanum tubingense (fig. 9 tu) a un épiderme de Tomate qui recouvre une charpente composée uniquement de tissus de Morelle et qui garde la forme propre à cette espèce ; $S$. Koelreuterianum présente les tissus internes, le port et le développement d'une Tomate recouverte d'un épiderme de Morelle ; mais Solanum proteus obtenu dans les mêmes conditions fournit un assemblage disparate où il est difficile de déterminer laquelle des deux espèces l'emporte sur l'autre; tour à tour, sclon les zones de croissance, les tissus de l'une éliminent pour partie les tissus de l'autre, ce qui donne lieu aux aspects les plus bizarres.

La cause profonde de ces alternatives échappe jusqu'à présent à l'analyse, parce qu'il est difficile d'en régulariser les effets. Il est probable, toutefois, qu'elle se rattache aux phénomènes d'excitation inhibitoire étudiés par L. Errera (1), dont l'exemple classique est fourni par les accidents traumatiques que les intempéries ou l'homme déterminent chez les Conifères. La régularité de croissance de la flèche des Pins, des Epiceas, des Sapins, la disposition en couronnes échelonnées de leurs rameaux secondaires, sont bien connues. Lorsque le vent brise la flèche d'un Pin, le

1. - L. Errera : Sur les conflits de préséance ou l'excitation inhibitoire chez les végétaux : Bulletin de la Société royale de botanique de Belgique (1904). 
plus vigoureux des rameaux latéranx de la couronne supérieure prend le dessus ; arqué et asymétrique jusque-là, il se dresse, donne en moins d'un an des bourgeons symétriques ; en un mot, il se substitue à la flèche et, dès qu'il a acquis la préséance manifeste, les désordres de croissance des rameaux voisins de même ordre cessent, leur répartition en couronne régulière se rétablit. En supprimant au fur et à mesure les flèches nouvelles, on met en évidence la tendance que possèdent tous les bourgeons à fournir un axe dressé. Il y a done bien action inhibitoire du bourgeon terminal qui donne la flèche sur les bourgeons qui donnent les couronnes de rameaux horizontaux, et eette action est d'autant plus forte que les bourgeons sont plus rapprochés les uns des autres.

Il arrive, rarement, que deux rameaux de la couronne qui suit la flèche détmuite sont de même force, et alors, tous deux deviennent des flèches qui se soudent après quelques années de vie indépendante, phénomène qui se produit dans la coalescence des tissus herbacés de la couronne mutilée des greffes de Solanum. La coalescence peut être réalisée entre masses trop irrégulières pour former un ensemble équilibré et on obtient les bourgeons S. proteus; l'équilibre est réalisé par l'indépendance relative des zones de croissance épider. raiques et profondes chez S. tubingense et S. Koelveuterianum de WinkLer.

Les Végétaux cryptogames ont une faculté de régénération earactéristique et il n'y a, pour ainsi dire, pas de fragment qui ne puisse, par une culture appropriée, réaliser presque instantanément la réparation et la restitution de l'organisme entier. Les Cryptogames vasculaires, dont les feuilles sont hautement différenciées, nous fournissent les 
exemples les plus nets des désordres de croissance intro duits par la mutilation. Après Figdor (1906), j'ai répété souvent l'expérience qui consiste à détacher la pointe d'une fronde de Scolopendre avec des ciseaux fins; les nervures latérales voisines de la section deviennent des nervures principales et on obtient une feuille à deux pointes. Répétant la
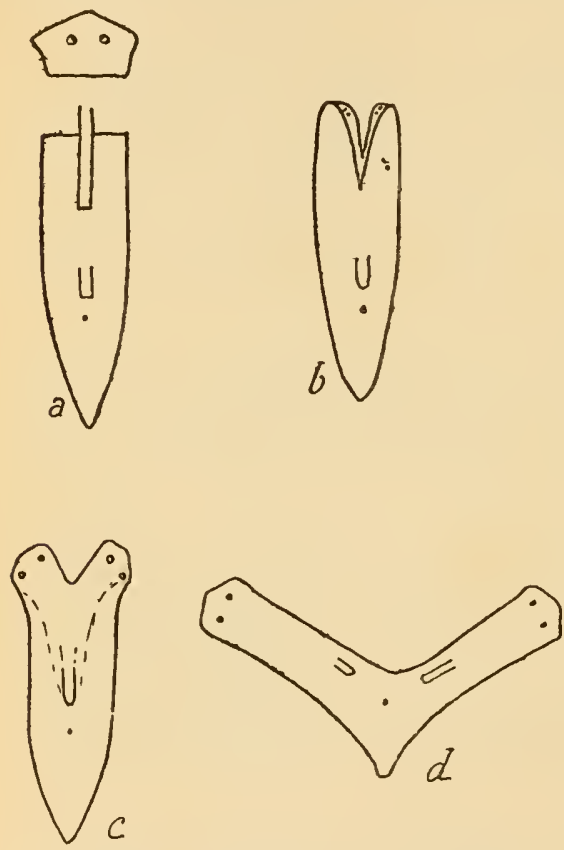

Fig. 10. - Section de la tête et entaille dans Planaria funebris; $a, b, c, d$, suite de formes au cours de la régénération (d'après Morgan). section sur ces pointes, il se forme des feuilles restaurées à quatre pointes et ainsi de suite. Il faut remarquer, en passant, que les Fougères en question ont donné, entre les mains des horticulteurs, rarement dans la nature, les multiples variétés à frondes crêtées ou dilacérées qui sont recherchées par les amateurs ; certaines se reproduisent par le semis.

L'étude des phénomènes ıe la régénération fut poussée à un haut degré de perfection dans le règne animal. Ici la symétrie des formes est si nette que les moindres altérations apparaissent; la plupart des embranchements : Coelentérés, Eohinodermes, Vers (fig. 10), Annélides, plus rarement les Ver- 
tébrés, donnent naissance à des individus aberrants lorsqu'on les mutile dans certaines conditions. Je n'insisterais pas davantage sur ces phénomènes si je n'y trouvais une indication précieuse de la variété et de l'étendue des connaissances de Pasteur. Ver's cette année (1857), il terminait, pour ainsi dire, ses études sur la dissymétrie moléculaire et commençait la publication de ses recherches sur les fermentations. A propos de la Génération spontanée, nous verrons l'importance qu'il accordait au débat qui avait mis en opposition, un siècle plus tôt, Spalimanzani et Needham. Or, SpalLANZANI, après 'TREMBLEEY sur les Hydres (1740), après Bonnet et Ríaumur (1741) sur le Vers, avait mis en évidence de très curieux phénomènes de régénération chez les Vertébrés (1768). Les Salamandres, dont les pattes et les appendices caudaux sont enlevés par une section radicale, régénèrent à partir de moignons et reconstituent l'organe, assez souvent modifié par rapport à l'organe synétrique conservé intact.

Il est possible que Pasteur ait été inspiré dans ses recherches sur le mode d'accroissement des cristaux par la lecture des œuvres d'un savant dont il admira la technique et dont il confirma les hypothèses ; mais, pour lui, la régénération n'est qu'un moyen d'étude du polymorphisme spéeifique; il fait d'ailleurs un rapprochement ingénieux entre les altérations dues aux mutilations et celles qui résultent de la présence d'impuretés dans les eaux-mères. Inspiré par la remarque d'A. LAUURENT rappelée plus haut (p. 15), Pasteur n'a cessé d'être préoccupé des eirconstances qui déterminent les formes anormales des cristaux. Dans sa thèse de doctorat (1847), il montre que la cristallisation de l'acide arsénieux est confuse lorsqu'on ajoute trop peu d'eau aux dissolutions : "Lorsqu'on sature une dissolution bouil- 
lante de potasse par de l'acide ar'sénieux jusqu'à refus, on obtient une liqueur très sirupeuse qui se dissout entièrement dans l'eau, véritable combinaisou, mais qui, peu à peu, se détruit par la présence même de cette eau, en laissant déposer beaucoup d'acide arsénieux;... il fant employer un volume d'eau trois ou quatre fois égal ou supérieur au volume du liquide sirmpeux » pour obtenir l'acide arsénieux cctaédrique. En 18t8, répétant une expérience de MITSCHERLICH, il obtient d'une même dissolution de sulfate de potasse cristallisé au sein d'une solution de carbonate de soude, d'abord du sulfate de potasse sous la forme dod'écaédrique ordinaire, puis, de l'eau-mère décantée et évaporée durant quelques jours, de gros cristaux en tables hexagonales appartenant au prisme hexagonal régulier.

La même préocupation persiste lor'sque PAsteur a mis en évidence la nature moléculaire de la dissymétrie liée à l'hémiédrie (1852) :

" J'ai pensé, dit-il, que dans le cas où la structure cristalline propre aux substances actives sur la lumière polarisée ne serait pas visiblement et géométriquement accusée, il suffirait de modifier les conditions de la cristallisation pour faire apparaître forcément et d'une manière constante les facettes hémiédriques. )) En chauffant le bimalate d'ammoniaque jusqu'à entraîner un début de décomposition et en le faisant cristalliser à nouveau dans l'eau, ou encore, en ajoutant à ane solution chaude de tartramide sur le point de cristalliser quelques gouttes d'ammoniaque, ou enfin, en faisant cristalliser le bitartrate d'ammoniaque dans une solution chargée de bitartrate de soude, il obtient à volonté les cristaux à facettes hémiédriques qui ne se forment pas dans d'antres conditions.

Et cependant les matières modificatrices sont exprulsées; 
elles n'agissent que par action de présence, souvent à l'état de traces; PASTEUR montre qu'elles altèrent la croissance.

L'intérêt de ces impondérables est mis de nouveau en Iumière par PASTEUR (1857) dans un Mémoire qui indique avec quelle attention il suivait les analogies des espèces eristallines et des espèces vivantes. La variété des formes cristallines d'un même corps chimique peut être " provoquée par la nature des substances qui avaient été présentes au moment de la cristallisation ") (1). Il ajoute aux preuves qui viennent d'être résumées le fait, connu depuis longtemps, que le sel marin cristallisé dans l'eau est cubique et, cristallisé dans l'urine, donne des octaèdres réguliers. Il s'efforce de prouver que ces modifications sont dues à des changements dans la vitesse de croissance des cristaux selon les différents axes de symétrie.

Le bimalate d'ammoniaque ordinaire, actif en solution sur la lumière polarisée, appartient au système du prisme à base rhombe et, dans les conditions ordinaires de cristallisation dans l'eau pure aux températures habituelles, présente deux formes (fig. 11, $a_{1}, a_{2}$ ); l'épaisseur est faible et le rapport des arêtes horizontales et verticales est très variable ; il y a un clivage très facile, parallêlement aux arêtes horizontales des figures, e'est-à-dire parallèlement aux arêtes des faces des biseaux multiples de la variété.

Si l'on brise un cristal (fig. 11, $a_{3}$ ) suivant un plan de elivage et qu'on le replace dans son eau-mère sursaturée, on voit un biseau se reformer avec une grande rapidité, avec zone restaurée très limpide si la reconstitution a été suffi-

1. - Etudes sur les modes d'accroissement des cristaux et sur les causes des variations de leurs formes secondaires. Ann. Ch. et Phys., $3^{e}$ sér., t. XLIX, 1857, p. 5. 
samment lente. A partir du moment où le biseau est reformé, le travail, qui jusque-là avait été d'une rapidité exagérée sur la partie supprimée, devient proportionnellement le même que sur le reste du cristal. En abattant à la lime un biseau latéral par une face normale à $P$, car il n'y a pas de clivage dans ce sens, ledit biseau se reforme encore avec une rapidité surprenante dans les mêmes conditions. Même résultat si on brise un angle du cristal selon la figure $11 a_{4}$ et ici on voit (fig. $11 a_{5}$ ) les zones d'accroissement progressif en lames courbes dont la régularité se rétablit à la fin lorsque les dernières traces de la blessure vont disparaître.

Tout se passe comme chez certains " êtres vivants lorsqu'on leur a fait une blessure plus ou moins profonde. La partie endommagée reprend peu à peu sa forme primitive, mais le travail de reformation des tissus est, en cet endroit, bien plus actif que dans les conditions normales ordinaires ".

PASTEur compare ces faits à ceux déjà connus que présente le bimalate d'ammoniaque ; formés dans l'eau pure, les eristaux ne portent jamais de faces hémiédriques ; mais si on retire les cristaux d'une eau-mère renfermant une petite quantité des produits d'altération, par la chaleur, de ce même bimalate d'ammoniaque, les cristaux ont les formes de la figure $11 a_{\mathrm{c}} a_{\mathrm{c}}^{\prime}$ et présentent deux faces hémiédriques $h$ qui sont quelquefois courbes. D'autre part, si l'on place des cristaux hémiédriques dans l'eau-mère pure qui jamais n'en donne, ils ne tardent pas à perdre toutes leurs faces hémiédriques. Inversement, si l'on transporte des cristaux sans faces hémiédriques formés dans l'eau pure dans la liqueur qui donne l'lémiédrie des cristaux, ils prennent peu à peu des facettes hémiédriques. L'action des impuretés sur la formation des faces est évidente. 

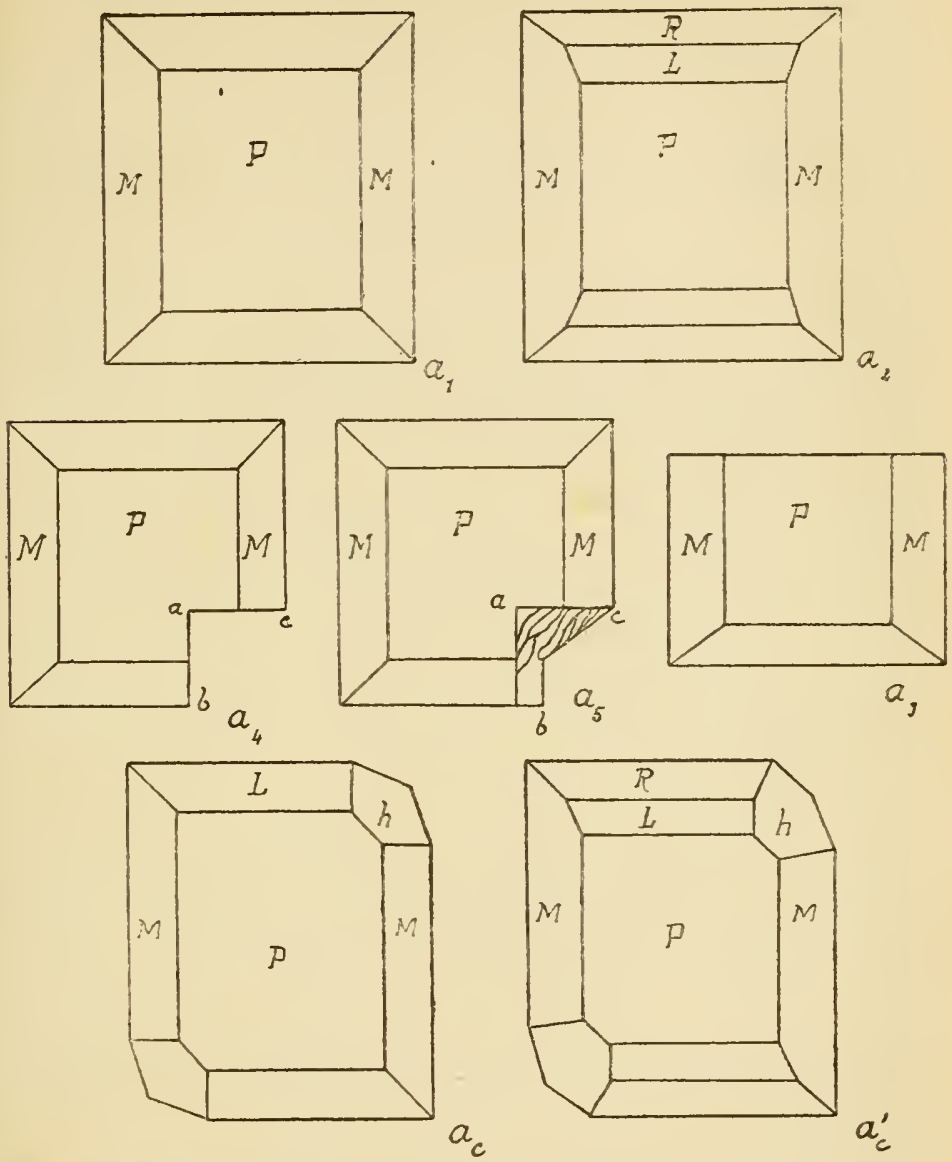

Fig. 11. - Formes cristallines du bimalate d'ammoniaque; $a_{1}, a_{2}$, formes fréquentes ; $a_{3}$, montrant le plan de clivage $; a_{4}, a_{5}$, en mutilation et régénération; $a_{c}, a_{c}$, formes en voie de croissance présentant les faces hémièdres, $h h$ (d'après PastruR). 
Le rôle des impuretés est done " d'altérer les rapports d'accroissement des cristanx suivant leurs diverses dimensions ». Autrement dit, elles modifient les conditions de nutrition. PASTEUR trouve, en effet, que les formes incomplètes, mutilées artificierlement, donnent des faces hémiédriques, exactement comme s'il y avait des impuretés dans l'eau de cristallisation, qui disparaissent avec le rétablissement du régime normal, tout comme les couches concentriques annuelles des arbres mutilés redeviennent normales après fermeture de la blessure : "Que l'on brise profondément un angle de bimalate d'ammoniaque non hémiédrique et qu'on place le cristal mutilé dans l'eau-mère pure qui jamais ne donne l'hémiédrie ; pendant tout le temps que se rétablira la cassure, on verra sur les diverses parties saillantes de celle-ci une ou plusieurs faces hémiédriques et d'autres faces secondaires qui n'ont pas ce caractère. Mais il n'y en aura plus trace une fois que l'angle sera rétabli, c est-à-dire dès que celui-ci aura repris le mode d'accroissement normal propre à la liqueur pure.

Inversement, précise PASTEUR dans une Note prélimiuaire (1), " dans une eau-mère pure de bimalate, j'ai placé un cristal non hémiédrique sur les faces latérales duquel j'avais collé de petites bandes de papier métallique, et dont j'avais abattu par clivage les biseaux supérieur et inférieur, double condition qui devrait rendre nul l'accroissement en largeur et très grand l'accroissement en longueur, et placer, par conséquent, le cristal dans la situation que fait naître la liqueur impure. Le lendemain, le cristal avait repris sa régularité, et les faces hémiédriques étaient accusées sur les

1. - Extrait par l'auteur communiqué à l'Académie des Sciences, 27 octobre 1856 , t. XLIII, p. 797. 
quatre angles solides. Cette expérience très démonstrative est délicate. Elle mérite d'être suivie avec beaucoup de soin, et il faut en quelque sorte prendre sur le fait la naissance des petites faces lémiédriques... Ces expériences paraissent appuyer fortement l'idée d'une étroite dépendance entre les modes d'accroissement des cristaux et la nature de leurs formes secondaires. »

Et Pasteur place dans le cour's de sa démonstration cette phrase caractéristique : "L'individualité d'un cristal ne réside pas dans sa matière propre avec sa forme visible et tangible; elle est tout entière dans la molécule chimique et dans les lois des distances des molécules chimiques identiques. " De même, chez les êtres vivants, l'individualité est dans l'œuf ; tont ce qui en résulte, larves, formes sexuées, adultes castrés par l'âge ou artificiellement, formes intermédiaires dues à la régénération après mutilation, toutes ces variations ne touchent pas à l'individualité spécifique ; elle réapparaît dans l'œuf et, au cours du développement, des circonstances convenablement choisies la rendent manifeste pour ainsi dire à volonté. PASTEur fournit de plus un moyen pour discerner la variation occasionnelle de la forme spécifique. "Dans ces expériences, il y a indépendance complète entre les diverses parties du cristal. Ce qui se passe sur un point n'a pas de rapport avec ce qui a lieu sur des parties semblables, mais éloignées. Tout paraît dépendre des conditions d'accroissement. „ Plus loin (p. 182), pour définir la lignée pure, nous devrons tenir compte des variations partielles, fluctuantes, locales, qui permettent de constater que l'individu vit dans des conditions favorables au développement de certaines particularités. Nous n'oublierons pas que des altérations, même très apparentes, dérivées ou non d'une même souche et suivies en deux 
lignées pures, ne doivent pas, pour cette seule raison, être réparties dans deux espèces différentes; elles peuvent être des manifestations différentes de la même individualité. Si, par divers procédés, on réussit à lamener l'une des lignées à ressembler à l'autre, autrement dit, si la variation est réversible (1), il faudra les classer dans la même unité spécifique.

L'espèce, selon la définition adoptée par PASTEUR, est la "collection de tous les individus identiques par la nature, la proportion et l'arrangement des éléments "; l'arrangement, ici seul en cause, n'est pas modifié par le fait que la forme visible est différente; il peut serulement y avoir eu des retards ou des accélérations dans le groupement des molécules.

La relation directe de l'inégale rapidité de nutrition des parties et de la forme était très délicate à mettre en évidence dans les phénomènes, en général, rapides de la cristallisation. Elle n'a pas échappé à l'investigation méticuleuse, allant au fond des choses, qui caractérise le génie de Pas'reur. Chez les êtres vivants, formés de colloïdes, les réactions sont infiniment lentes; elles retiennent l'attention et constituent le polymorphisme. Il n'y a entre les denx règnes qu'une diffé. rence de durée dans l'acquisition de l'état stable. La grande majorité des modifications des êtres vivants, mises en valeur par les transformistes, doivent être examinées d'après cette conception; le milieu, les impuretés, l'âge, les mutilations, ont des effets très différents de l'altération spécifique proprement dite, dont nous allons étudier les caractères.

1. - Voir L. Blaninghem : Les problèmes de l'Hérédité expérimentale. Paris, E. Flammarion, 1919 ; Introduction. 


\section{CHAPITRE III}

\section{HÉRÉDITÉ, HYBRIDATION ET MUTATION}

L'étude de la variation fournit, par contraste, la délimitation des éléments stables dans la sous-espèce et dans l'espèce elle-même. Les cristallographes tiennent compte depuis ROMÉ DE LISLE (1783) d'une forme primitive qui, par la

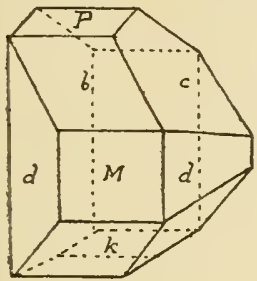

$d r$

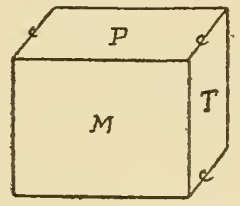

$\int p$.

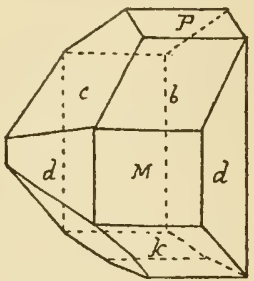

ga.

Fig. 12. - Forme primitive $f p$ de l'acide tartrique; $d r$, cristal hémièdre droit ; $g$ a, cristal hémièdre gauche.

troncature des angles et par l'addition de facettes supplémentaires, fournit les formes les plus variées rencontrées dans la nature (fig. 12). Après les remarquables travaux de l'abbé Haüy, il devient évident que l'essentiel de la forme primitive était dans les molécules intégrantes très petites qui, s'empilant les unes sur les autres, mais en nombres divers, fournissaient l'illusion de la troncature ou du biseautage de la forme primitive. En réalité, nous l'avons vu par la régénération des cristaux, ce n'est pas la suppression des parties sur une forme primitive massive qui donne nais- 
sance au polymorphisme, c'est plutôt l'arrêt de croissance, c'est-à-dire la limitation, par des forces extérieures au corps lui-même, du nombre des molécules se groupant pour faire une masse cristalline.

Il résulte de là que l'essentiel de l'espèce, quant aux caractères de forme et de symétrie, peut rester caché ou inhibé clans son développement et on obtient les variétés par latence, les variétés par défaut, ete., qui constituent une bonne partie des faits classés par les tératologistes. MoQUIN T'ANDon (1841), après avoir étudié la symétrie végétale, adopte la définition zoologique de ces irrégularités donnée par Isidore Geoffroy-SaInT-Hilaire (1830). “Outre le type primitif ou symétrique et le type de famille régulier ou irrégulier, il existe encore un troisième type qui a reçu le nom de spécifique : c'est l'ensemble des traits, la réunion des caractères communs à la plupart des individus qui composent une espèce. Toute déviation de ce type, ou, en d'autres termes, toute particularité organique que présente un individu, comparé à la majorité de ceux du même nom, donne naissance à une anomalie. )

Sont des anomalies l'avortement des étamines de l'Eglantier, avec métamorphose des pièces correspondantes en organes pétaloïdes qui forment les Roses doubles, et aussi l'absence de pigment des corolles du Lilas blanc. Ces particularités sont des aceidents, des raretés, et si l'on n'avait pris soin de les multiplier par boutures ou par greffes, souvent aussi par le semis lorsqu'il s'agit de variétés de plantes annuelles, ces aberrations disparaitraient et seraient presque introuvables dans la masse des individus normaux. De plus, elles persistent d'ordinaire avec lcurs caractères tranchés, indépendamment des circonstances immédiates de leur élevage; elles présentent après isolement une fixité souvent 
plus marquée que celle des attributs permanents de l'espèce ; e'est pourquoi elles servent de réactifs pour l'analyse des caractères spécifiques. Nous allons étudier, dans ce chapitre, comment se perpétuent et, en partie, comment naissent les anomalies héréditaires.

Il est difficile de mettre en pleine lumière la conception de Pasteur relative à l'hérédité. Elle consiste évidemment dans la permanence de la nature et des proportions des éléments qui définissent l'espèce chimique. Mais n'a-t-il pas laissé entrevoir son opinion relative à la nécessité d'une permanence de l'arrangement moléculaire fondamental ?

Je crois l'avoir trouvée dans l'avant-propos du célèbre Mémoire sur la fermentation appelée lactique (1). PASTEUR est toujours sur la piste des dissymétries moléculaires qu'il déinit par la polarisation rotatoire des corps en dissolution; il s'assure que la déviation de la lumière subsiste à travers certains dérivés d'ordre chimique qui présentent des affinités par la nature et la proportion de leurs éléments. Où s'arrête la permanence de la dissymétrie moléculaire ? "La constitution moléculaire des sucres, dit-il, me paraît très différente de celle de l'alcool amylique. Si cet alcool, lorsqu'il est actif, avait le sucre pour origine, comme tous les chimistes l'admettent, son action optique serait empruntée à celle du sucre. C'est ce que je répugne à croire dans l'état actuel de nos connaissances, parce que toutes les fois que l'on essaie de suivre la propriété rotatoire d'un corps dans ses dérivés, on la voit disparaître promptement. Il faut que

1. - Annales de Chimie et de Physique, 3e sér., t. LII, 1858, p. 405 , et séance du 3 août 1857 de la Soc. Sc. ag. de Lille. 
le groupe moléculaire primitif se conserve en quelque sorte intact dans le dérivé pour que ce dernier continue d'être actif. "

La première série de preuves, et la plus simple, de la permanence d'une dissymétrie moléculaire dans la formation des corps dérivés est fournie par la démonstration de l'existence de l'hémiédrie dans tous les sels obtenus de l'acide tartrique ordinaire droit. La composition chimique varie, mais le caractère fondamental de la polarisation rotatoire subsiste dans le même sens et avec la même intensité. Ces faits sont exposés avec la plus grande clarté dans la seconde Thèse de doctorat ès sciences (1) de PAsteur (1847). A la vérité, ils résultaient des travaux de Mitscherlich sur l'isomorphisme et de ceux de Віот, sur l'acide tartrique et ses combinaisons.

Pasteur vérifie les faits ; il étudie les dissolutions de deux sels où ne se précipite aucun sel; il mesure les déviations pour des concentrations données et constate que le tartrate double de soude et de potasse se comporte autrement que le simple mélange (en poids équivalents anx constituants du tartrate double) de tartrate de soude et de tartrate de potasse. La combinaison possède un pouvoir de déviation propre; le mélange donne exactement la moyenne arithmétique des déviations des deux tartrates simples. En effet, les solutions renfermant sous le même volume des poids de sels proportionnels aux équivalents chimiques de ces divers tartrates donnent : $23^{\circ}, 6^{\prime}$ pour le tartrate de soude, $30^{\circ} 5^{\prime}$ pour le tartrate de potasse, $26^{\circ} 67^{\prime}=\frac{23^{\circ} 6^{\prime}+30^{\circ} 5^{\prime}}{2}$ pour le

1. - Application de la polarisation rotatoire des liquides à la solution de diverses questions de chimie. Paris, Bachelier. 
mélange à volumes égaux des deux tartrates simples et $30^{\circ} 3$ pour le tartrate double de potasse et de soude. " Il faut en conclure, dit PASTEUR, que dans le mélange en question le sel double ne prend pas naissance. " Nous pouvons dire de même que l'hérédité n'est parfaite que là où il n'y a pas combinaison.

Poursuivant sa démonstration, Pasteur prouve que les molécules des corps isomorphes (́tartrate de potasse avec tartrate double de potasse et d'ammoniaque) ont le même pouvoir de déviation sur la lumière polarisée. Je ne donne pas de chifïres, puisque le fait est bien connu, mais je cite intégralement l'explication fournie : "Il résulte de là que, dans le tube d'observation, nous avons sur une même longueur le même nombre de molécules ; et, puisque nous trouvons la même déviation, nous pouvons énoncer le résultat de cette expérience en disant que les molécules de deux corps isomorphes dévient de la même quantité le plan de polarisation des rayons lumineux. " La même règle est vérifiée pour l'émétique de potasse et l'émétique d'ammoniaque. Elle est valable pour les tartrates gauches, pour les paratartrates.

Nous nous trouvons, en ce moment, exactement dans les conditions où s'appliquent les lois de l' hérédité alternante, découvertes par MendeL, en croisant entre elles certaines variétés de Pois (1865), par M. DE VRIES, en croisant entre elles certaines variétés de Maïs (1900). Les corps ammoniaque, potassium, sodium, n'altèrent pas la dissymétrie moléculaire propre à la molécule de l'acide tartrique. On peut échanger un atome de sodium contre un atome de potassium sans que la polarisation rotatoire propre à l'acide tartrique soit modifiée; on est done en droit de dire que chez 
les composés isomorphes la polarisation rotatoire de l'acide tartrique est indépendante de la nature de la base ; les bases s'échangent selon les règles de la chance et du hasard, e'està-dire par parties égales lorsque les corps sont mélangés par parties égales.

De plus, on se rend compte de la signification qu'il faut attribuer aux mots dominance, récessivité, dans les composés formés de deux bases. Le tartrate double de potasse et de soude est un sel hétérozyggote qui présente, comme un hybride entre le Maïs amylacé et le Maïs sucré, le caractère marqué de 1'un d'eux ; ici, le sel double offre une polarisation rotatoire $\left(30^{\circ} 3\right)$ à peu près égale à celle du sel de potasse pur $\left(30^{\circ} 5\right)$; le caractère imprimé par l'atome potassium domine et reste seul apparent. Mais on peut imaginer des cas, et les sels isomorphes en fournissent de nombreux exemples, où la dominance est incomplète et où la polarisation rotatoire du sel double est intermédiaire. Certains sels peuvent même avoir des dominances inversées, selon les modes de préparation ou leurs origines (impuretés), de mênze que chez les Pois, où d'ordinaire les cotylédons jaunes dominent les cotylédons verts, il arrive que le contraire ait lieu (1). L'essentiel de l'espèce n'est pas modifié ; la molécule cristalline a gardé intacte sa dissymétrie; elle est à peine touchée, guère plus que ne le ferait une impureté modifiant la viscosité des solutions, par la présence ou par l'échange de tel ou tel corps inactif (potassium, sodium, ammoniaque) ; elle reste indépendante de la substitution de ces corps entre eux.

Dans la formation des eristaux, on dit qu'il y a isomor-

1. - L. Blaringhem : Hérédité anormale de la couleur des embryons d'une variété de Pois, C. R. Ac. Sc., t. CLXXV, 1922, p. 877. 
phisme; la forme, la taille des cristaux ne sont pas sensiblement altérées, mais il apparaît des facettes diverses qui traduisent des modifications dans la nutrition, dans la rapidité du dépôt. Dans les croisements strictement mendéliens, les générations successives ne diffèrent guère par la vigueur, par le port ; les caractères végétatifs sont indépendants des couples de caractères qui se substituent les uns aux autres sans entraîner d'altération dans la fécondité.

$$
* *
$$

Le moment est venu de faire connaître les définitions des divers degrés de spéciéité d'après Charles Naudin (1858). Contemporain de PASTEUR, moins favorisé par la fortune, il dut accepter un emploi de jardinier an Muséum d'Histoire naturelle de Paris pour compléter ses études préparatoires et poursuivre ses premières recherches. Lui aussi séria les faits et constata que les Courges (Cucumis), que les Potirons (Curcurbita) forment des séries parallèles de types ayant les mêmes formes, les mêmes aptitudes, si voisines qu'il faut une longue pratique pour les distinguer. Une différence essentielle lui apparaît : le pollen d'une Courge quelconque ne féconde jamais l'ovaire d'un Potiron quelconque et, par ce caractère physiologique, il sépare les formes et les range dans leurs genres respectifs.

Ses recherches sur les hybrides le conduisent, en 1858, à la définition physiologique de l'espèce chez les végétaux supérieurs : " eollection des individus, quelqnes dissemblables qu'ils soient par le faciès, qui peuvent se féconder et par là donner naissance à une postérité indéfiniment féconde, qui conserve dans toute la série des générations les traits propres à chacun des deux premiers ascendants dont elle 
est issue, à moins que de nouveaux croisements n'en vienwent troubler la transmission »(1).

D'ailleurs, il y a des degrés de spéciéité relative; la spéciéité est :

Du premier degré, lorsque les deux plantes comparées ne peuvent jamais se féconder réciproquement [Poirier et Pommier, Melon et Concombre (NAudin); Lin à fleurs jaunes et Lin à fleurs bleues (BLARINGHEM) ] ;

$D u$ deuxième degré, lorsque les deux plantes, pouvant être à la rigueur fécondées l'une par l'autre, l'hybride qui en résulte non seulement est stérile par lui-même, mais résiste à l'action des pollens du père et de la mère [Nicotiana rustica et $N$. californica (NAUDIN) ; Digitalis purpurea et D. lutea, Seigle et Epeautre (Blaringhem) ] ;

Du troisième degré et caractérisée par la possibilité de féconder l'hybride par le pollen des deux parents, ou au moins de l'un d'eux, bien qu'il soit stérile par son propre pollen [Nicotiana augustifolia et N. glauca (NAUDIN); certains Verbascum, Dianthus et Geum hybrides, Seigle et Tri. ticum turgidum (BLARINGHEM)] ;

Du quatrième degré et caractérisée par la fertilité plus ou moins grande pendant un nombre limité de générations, retournant sans s'éteindre au type de l'un des deux parents par l'élimination graduelle des caractères de l'autre [Primula veris et $P$. suaveolens (NAUDIN); Triticum Monococcum et $T r$. durum, Linaria vulgaris et L. striata (BLARINGHEM) ] :

$D u$ cinquième degré, lorsque les deux espèces comparées se croisent avec facilité et que leur descendance, aussi

1. - Сн. Naudis. Quelques considérations sur l'espéce et la variété : modification proposée à la définition de l'espèce en botanique, C. R. Acad. Sciences, 15 février 1858, t. XLVI, p. 340. 
féconde qu'elles-mêmes, se perpétue indéfiniment sans rentrer d'une manière complète dans les types maternels ou paternels [Petunia nyctaginiflora et $P$. violacea (NAUdin): divers Blés entre eux, Geum urbanum et $G$. rivale, Primula officinalis et $P$. veris (BLARINGHEM)].

Plus loin, NAUdin expose clairement l'idée qu'il se fait du transformisme: "Le fait, incontestable aujourd'hui, de la division des espèces en variétés permanentes, subdivisées elles-mêmes en variétés secondaires qui sont aux premières ce que l'espèce est au genre, ouvre de nouveaux aperçus à l'esprit. On se demande naturellement d'où viennent les analogies qui ont fait réunir des espèces distinctes en genres et en familles. ") Ce ne peut être dû au hasard et e'est sans doute " que tout ce qu'il y a de commun entre les espèces d'un même groupe naturel a été puisé à une source commune. Ceci revient à dire que les espèces d'un même genre ou d'une nême famille sont autant de formes dérivées dont le type primordial s'est successivement divisé dans le cours des âges »).

Dans cette dernière phrase seulement, NaUdin, contemporain de Darwin et de Pasteur, se sépare de Pasteur pour suivre Darwin. NAUdin avait énoncé le premier (1852) l'hypothèse $d$ 'une filiation naturelle et matérielle des espèces selon les ramifications d'un arbre généalogique ; très instruit dans les sciences physiques et chimiques et, par conséquent, au courant des découvertes pastoriennes, il connaît mienux que quiconque, par ses propres études et par les rapports suivis qu'il entretient avec Jordan, que dans la nature il n'y a que des sous-espèces. L'espèce de LinNé, les genres, les familles sont des divisions théoriques qui ne cadrent pas du tout avec la notion expérimentale de la spécieiité, dérivée de l'épreuve des croisements et des semis successifs. Logi- 
qucment, il aurait dû, aux côtés de Pasteur, contre Darwin, défendre la permanence de l'espèce.

Et voilà bien, à mon avis, la part d'influence, sur le développement du génie, de la matière sur laquelle portent les premières recherches, celles qui modèlent le cerveau, $\mathrm{y}$ impriment des liaisons, résultat de la répétition des groupements de faits. Pasteur étudie des matières inertes, des cristaux à composition définie pondéralement, đes solutions ayant une existence éternelle, ubiquiste par rapport au temps et à l'espace à travers lesquels s'échelonnent les séries de faits examinés par Naudin. Les espèces de Courges, de Tabacs, de Linaires que Naudis étudie, ont été rassemblées de tous les pays du monde et les matériaux rémnis sont l'image d'une succession dans le temps des formes dérivées d'un même type primordial; en cherchant à dégager les points communs à toutes ces formes, il ne peut éliminer les circonstances de leur acquisition. En un mot, il n'y a transformisme que là où le temps et l'espace échelonnent les liaisons entre les phénomènes. Il n'y a pas de transformisme dans la cristallisation ni dans la polarisation rotatoire, où les notions de durée et de lieu n'ont rien à faire. Il ne paraît pas y en avoir davantage dans la science des microorganismes, bien qu'il s'agisse d'êtres vivants cette fois, parce que la sériation des ferments et des microbes ne se fait ni d'après leur échelonnement dans le temps, ni d'après leur répartition dans l'espace ; elle se fait d'après la nature des bouillons de culture, qui multiplient à l'infini, en quelques heur'es, l'unique cellule que Pasteur y dépose, ou qui détruisent, sans rénovation possible, les germes de ces espèces.

On peut encore dire, si l'on veut, qu'il y a un transformisme spécial aux formes cristallines d'une série de com. posés chimiques de même famille, un transformisme spécial 
aux microorganismes, un transformisme spécial aux formes vivantes réparties dans l'espace et dans le temps. Mais, du point de vue logique, comme du point de vue expérimental d'ailleurs, l'homme opérant dans un espace limité et avec une durée limitée n'accumulera, comme preuves de la métamorphose, que des faits de l'ordre de ceux que lui offrent les arrangements moléculaires, les substitutions d'éléments ou les interréactions des espèces chimiques; ces métamorphoses paraissent toujours bmusques, discontinues et irréversibles, par conséquent, du type des réactions chimiques; elles ne peuvent rien prouver, ni pour ni contre un enchaînement des organismes par étapes, à la façon des branches de l'arbre généalogique imaginé par NAUdis, à moins que, et je suis en ce moment en pleine hypothèse, on ne découvre des lois de sériation obligatoire dans l'addition des molécules pour passer de charpentes moléculaires simples à l'édification de charpentes aussi complexes et aussi peu stables que les complexes albuminoïdes; certaines découvertes réalisées dans la chimie synthétique nous permettent de penser que là gît l'essentiel du problème du transformisme commun aux diverses substances.

Nous verrons, d'autre part, que les soi-disant mutations de microbes sont presque toujours réversibles à volonté (p. 235) et traduisent, par conséquent, une tout autre caté. gorie de phénomènes.

Sur elles reposent les principes fondamentaux de la vaceination et de l'analyse, si parfaite depuis quelques décades, de la valeur relative des parties constituant l'alimentation, tant des microbes et des cristaux que des espèces supérieures et de l'homme lui-même. Nous en reportons l'étude dans la seconde et la troisième parties de cet ouvrage. 
LiNNÉ (1760) a eu l'intuition que des espèces nouvelles pouvaient naître de l'hybridation de deux espèces distinctes ; il n'a pas réussi à convaincre ses contemporains, mais il a provoqué des épreuves de croisement, surtout chez les plantes cultivées, qui ont abouti à de remarquables résultats pratiques. Pour les horticulteurs, la production d'hybrides intermédiaires plus ou moins stables n'est pas douteuse. Cependant Naudin n'accepte pas cette interprétation; il établit (1863) par des épreuves remarquables que l'hybride est une mosaïque vivante d'éléments spécifiques différents, conservant leur mode ‘de végétation particulier, sans cesse en lutte pour se dégager les uns des autres. Il arrive [Datura, Linaria, Mirabilis (Naudin), Hordeum, Cardamine, Pisum (BuARINGHEM)] que la séparation se produise dans certaines parties localisées de l'hybride, rameaux et feuilles, mais la tendance s'accroît toujours avec l'âge et se manifeste surtout dans les fleurs et sur les fruits. " Ces faits autorisent à penser, déclare NAUDin, que le pollen et les ovules, le pollen surtout, qui est le terme extrême de la floraison mâle, sont précisément les parties de la plante où la disjonction spécifique se fait avec le plus d'énergie. »

Supposons la disjonction faite à la fois dans l'anthère et dans le contenu de l'ovaire; le tube d'un grain de pollen revemu à l'espèce du père rencontrant un ovule disjoint dans le même sens produira une fécondation parfaitement légitime, dont le résultat sera une plante retournée à l'espèce paternelle; la combinaison du pollen et de l'ovule disjoints dans le sens maternel donnera un produit strictement maternel ; la combinaison de pollens et d'ovules disjoints en sens 
contraire l'un de l'autre reconstituera l'hybride premier. NAUdin vérifie, par des épreuves nombreuses, qu'il en est souvent ainsi. Il n'écarte d'ailleurs pas l'hypothèse de pollens et d'ovules mixtes, mais il affirme que, tôt ou tard, ces complexes se désagrègeront, et e'est, en effet, le cas le plus fréquent. NaUdin nie done la possibilité de fixer un hybride mixte.

Actuellement, on doit encore admettre que, dans les rares cas où cette fixation paraît bien établie (Geum intermedium (1), Primula variabilis, Monococcum $\times$ Triticum durum), la fixité n'est qu'apparente; les éléments sexuels mixtes subsistent, mais sont instables au point que les lignées qui en dérivent sont sujettes à des retours inattendus et présentent toujours une fécondité réduite. En définitive, la production de germes mixtes se transmettant tels est exceptionnelle; les pseudo-espèces apparues à la suite de 1 'hybridation peuvent se reproduire par voie sexuée durant des années ; leur propagation dans le temps et dans l'espace sera tôt ou tard suivie de la régénération des types ancestraux plus stables; on doit les traiter comme ces complexes chimiques étudiés par PaSTEUR, qui sont des positions d'équilibre entre les molécules, et non comme des édifices moléculaires nouveaux.

N'oublions pas, d'ailleurs, que les facteurs physiques, de même que les impuretés, qui altèrent les rapports de la croissance des cristaux ou déterminent l'apparition de faces supplémentaires, peuvent être constamment actifs et donner aux phénomènes une apparence de nouveauté accompagnée de stabilité. Leur permanence n'est pas comparable à

1. - Blaringhem : Stabilité et fertilité de l'hybride Geum urbanum $\times$ G. rivale, C. $R$. Acad. Sc., t. CLXX, p. 1284. 
la transmission des véritables attributs de l'espèce, telle que la permanence de la dissymétrie moléculaire à travers les substitutions de corps isomorphes. Les épreuves basées sur les règles de Mendel séparent d'ordinaire les hybrides complexes des espèces types avec la plus grande facilité.

Nous trouvons done un guide précieux, dans l'analyse compliquée des phénomènes offerts par les espèces vivantes, en suivant PaSTeur dans ses études sur la permanence ou sur les altérations de la dissymétrie des espèces chimiques. Je passe sous silence, bien que très suggestif, le fait que le glucosate de sel marin, dissous dans l'eau, offre des variations avec le temps, allant presque du simple au double, dans les pouvoirs rotatoires des dissolutions. Pasteur constate que lorsque la variation cesse, la solution se comporte comme si elle était formée de glucose pure; e'est un retour à l'espèce active, selon l'hypothèse de Naudin.

Les faits sur lesquels je désire retenir l'attention sont plus généraux. Pasteur les expose sous le titre: Sur une nouvelle classe de combinaisons isomères, actives sur la lumière polarisée (1) et nous avertit, dans le préambule, qu'il faut distinguer les combinaisons des espèces actives avec des substances inactives, et les combinaisons des mêmes espèces actives avec des substances actives, elles aussi. On comprendra l'intérêt que j'attache à ce Mémoire en constatant que je compare la stabilité des croisements entre formes, lignées, variations d'une même espèce aux premières, et l'instabilité des hybrides entre espèces différentes aux secondes combinaisons ; deux espèces distinctes vivantes

1. - Annales de Chimie et de Physique, 1853, $3^{\text {e }}$ sér., t. XXXVII, 2e partie du Mémoire Nouvelles récherches. 
ont, dans mon hypothèse, des dissymétries moléculaires propres (1).

"La cristallisation d'un mélange de bitartrate d'ammo. niaque droit et de bimalate d'ammoniaque actif, dit PASTEUR, donne lieu à plusieurs remarques fort curieuses.

"Si l'on fait un mélange des deux sels à équivalents égaux, et qu'on le dissolve à chaud dans beaucoup d'eau, la première cristallisation obtenue par refroidissement est entièrement composée de bitartrate d'ammoniaque droit, pur, en lames carrées assez larges, biselées sur les bords. L'eau-mère, après évaporation et refroidissement, fournit une nouvelle cristallisation homogène, en prismes fort peu nets, dont il est impossible d'assigner la forme exacte, et terminés par une pointe très aiguë. Je les appellerai, pour abréger, cristanx (B). La nouvelle eau-mère, traitée de la même manière, fournit des cristaux groupés en mamelons blancs, tuberculeux; je les appellerai (C). Enfin, la nouvelle eau-mère donne uniquement, et jusqu'à la dernière goutte, du bimalate d'ammoniaque pur ou à peine souillé de cristaux (C). "

C'est le modèle, simplifié à l'extrême, d'une disjonction

1. - J'ai insisté tout spécialement sur ce point dans la conférence du 22 novembre 1922 à l'Ecole Normale. Au début de janvier 1923, j’ai eu connaissance dans les Euvres de Pasteur, réunies par M. Pasteur Vallery-Radot, $t$. I, p. 241, d'un complément au Mémoire analysé ici : "Pour se rendre compte de la formation exclusive de molécules d'un seul ordre de dissymétrie, il suffit d'admettre qu'au moment de leur groupement les atomes élémentaires sont soumis à une influence dissymétrique, et comme toutes les molécules organiques qui ont pris naissance dans des circonstances analogues sont identiques, quels que soient leur origine et le lieu de leur production, cette influence doit être universelle. Elle embrasserait le globe terrestre tout entier. A elle serait due la dissymétrie moléculaire des produits naturels des végétaux, pro. duits que nous retrouvons chez les animaux à peu près sans altération et où ils jouent un rôle mystérieux duquel nous n'avons encore aucune idée. " Extrait d'un ouvrage projeté de L. Pasteur sur la Dissymétrie moléculaire et non publié. 
en mosaïque dans un hybride interspécifique (Cytisus, Hordeum, Linaria). L'épuration des deux types commence dès le début du refroidissement. D'autre part, les intermédiaires (B) et (C) sont, Pasteur le prouve, des combinaisons à équivalents égaux de bimalate d'ammoniaque et de bitartrate d'ammoniaque.

Si on diminue la quantité d'eau à la limite approchée de la quantité nécessaire pour la dissolution à chaud, il se forme un assemblage bizarre des deux espèces de cristaux :

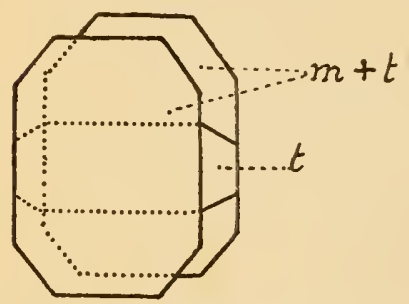

Fig. 13. - Mosaïque cristalline dans la cristallisation de bimalate d'ammoniaque et de bitartrate d'ammoniaque; le cristalt horizontal est un tartrate pur; les cristaux allongés $m+t$ sont des complexes de bimalate et de bitartrate (d'après PAsteur).

" tous, sans exception, sont formés, comme l'indique la figure 13, de deux lames identiques, qui comprennent entre elles une autre lame de même largeur, mais de hauteur moindre..., le cristal couché intérieur est du bitartrate d'ammoniaque parfaitement pur, et les lames latérales sont la combinaison à équivalents égaux de bimalate et de bitartrate d'ammoniaque, identique, malgré l'aspect de la cristallisation, avec les cristaux (B) et les cristaux (C)... Cette combinaison n'est pas stable, c'est-à-dire qu'on ne peut la faire cristalliser sans la détruire en partie. ”

On ne peut mieux illustrer, par un exemple précis, l'hypothèse de NAUDIN relative à la mosaïque hybride, si nette, dans Cytisus Adami (1).

1. - Blaringhem : Sur l'hérédité en mosaïque. Congrès internat. de Génétique, Paris, 1911, 40 pages et 23 figures. 
Les expériences de PASteur, réalisées avec les combinaisons de tartramide droite et malamide active, de tartramide ganche et malamide active, font apparaître une autre loi. "Les corps droits et gauches non superposables, dont les propriétés physiques et chimiques sont d'une identité absolue quand on les unit à des substances inactives sur la lumière polarisée, se comportent d'une manière toute différente dès qu'on les met en présence de produits actifs. "

Le fait est général; il ne résulte pas de l'affinité spéciale qui unit l'acide malique à l'acide tartrique; PASTEUR le vérifie pour les tartrates droits et les tartrates gauches de cinchonine, corps n'ayant aucune propriété commune, et de constitutions moléculaires totalement différentes ; mêmes résultats avec les tartrates droits et gauches de brucine, de strychnine, et, à propos de ces dernier's, PASTEUr met en évidence le mécanisme physique de ces altérations. Les tartrates acides de strychnine ont exactement la même composition chimique... Le tartrate gauche laisse échapper l'eau de cristallisation à $100^{\circ}$, bien plus vite que le tartrate droit ; à cette différence dans l'énergie de liaison avec l'eau de cristallisation correspondent des formes cristallines qui ne peuvent dériver d'une niême forme primitive.

D'où la loi générale : “ Les corps droits et les corps gauches non superposables, dont l'identité chimique est absolue tant qu'on leur offre des molécules inactives sur la lumière polarisée, se comportent comme des êtres tout à fait distincts dès qu'ils sont en présence de molécules qui, ellesmêmes, sont actives. ”

J'en déduis, avec quelque raison, que les règles de l'hérédité alternante - qui s'appliquent dans le cas où les formes croisées ne diffèrent que par des caractères indépendants de la dissymétrie moléculaire, et présentent alors une 
rigueur telle que les résultats sont prévus d'après les règles du calcul des probabilités - ne peuvent être utilisées sans autre précaution dans le domaine des hybrides interspécifiques, où j'observe la mosaïque des caractères et divers ajustements plus ou moins complexes, variables dans chaque cas spécial, parce que les dissymétries combinées varient précisément avec les espèces croisées (1).

Suivons Pasteur dans ce domaine particulier, et nous assisterons à la création, pour ainsi dire artificielle, d'espèces pourvies de propriétés physiques nouvelles. Il y fut conduit par la constatation du fait que les combinaisons des acides droits et des acides gauches avec la même base organique active ne sont plus ni également solubles, ni également hydratées ; même lorsque les formules de constitution chimique sont identiques, leurs formes cristallines diffèrent au point d'être incompatibles. Parfois, l'une des combinaisons est possible, l'acide tartrique droit avec l'asparagine, par exemple, tandis que la combinaison de la même base avec le corps gauche est irréalisable. J'ai montré (2) que le croisement du Seigle avee le Blé Epeautre échoue en pollinisant le Seigle par le Blé ; elle n'est possible qu'en prenant le Blé comme plante-nère et le Seigle comme père ; la combinaison est d'ailleurs stérile et donne lieu à des modifications de la croissance telles que l'hybride construit huit fois le poids de la matière sèche des parents cultivés dans

1. - Le lecteur trouvera dans une Revue que j'ai publiée, à la demande d'Y. Delage, dans l'Année biologique 1920, la distinction des hypothèses fondamentales du Mendelisme et du Naudinisme.

2. - Sur un hybride stérile d'Epeautre et de Seigle, $C$. R. Académie Sciences, 16 octobre 1922, p. 635. 
les mêmes conditions et durant le même temps ; les hybrides analogues sont fréquents.

Duclaux, dans l'Histoire d'un Esprit, présente les hypothèses directrices de PASTEUR sous une forme condensée que je lui emprunte (p. 57) : "Lorsque nous combinerons deux corps dont chacun aura son droit, son gauche et son inactif, nous pourrons avoir neuf combinaisons diverses, identiques quant au nombre et à la nature des atomes, mais différentes par leurs arrangements. Cette différence d'arrangement comportera l'adjonction d'un nombre inégal de molécules d'eau de cristallisation, qui seront plus ou moins difficiles à chasser par la chaleur. Elle entraînera en outre des différences dans la forme eristalline, la solubilité, la stabilité chimique.... Les différences qu'on observe entre les diverses combinaisons sucrées rencontrées dans la nature sont évidemment du même ordre et ont la même origine. J'oserai ajouter que ce sont des raisons de même nature qui rendent si inextricable l'étude des matières albuininoïdes... Le protoplasma de toutes les cellules vivantes est doué du pouvoir rotatoire; il contient par suite des molécules dissymétriques, et cette dissymétrie, en relation avec la stabilité ou l'instabilité du composé, ne peut manquer de jouer un rôle dans toutes les combinaisons chimiques dont le protoplasma est le siège. On concluera qu'il y a dans ces considérations l'indication d'un mécanisme profond de la vie. ")

Reprenons pied dans le domaine expérimental. En chauffant le tartrate droit de cinchonine, PASTEUR en retire l'acide paratartrique; en combinant la cinchonicine avec l'acide paratartrique, il voit se déposer du tartrate gauche de cinchonicine, le tartrate droit restant dans la liqueur. Voilà donc le procédé qui permet de passer d'une forme de dissymétrie moléculaire à une autre ; d'une sous-espèce définie 
à une autre sous-espèce définie. Il réussit rarement on du moins les circonstances qui permettent cette transmutation sont relativement rares, et e'est pourquoi la stabilité de la sous-espèce apparaît dans la très grande majorité des cas comme tout à fait garantie. Remarquons que rien, dans l'espèce chimique proprement dite, n'est changé ; elle constitue un groupement d'unités d'ordre supérieur qui reste indifférent parce que les combinaisons nécessitent l'affinité et, comme NAUdin le laisse deviner, là où l'affinité manque, les combinaisons hybrides ne se réalisent pas; le Pommier ne féconde pas le Poirier. Le Pommier, le Poirier, sont les v raies espèces d'ordre supérieur qui, en l'état actuel, limitent à leur intérieur les mutations et les altérations. On conçoit aussi qu'il n'y ait plus de règle à portée générale, indépendante des objets; il s'agit de cas qui doivent être étudiés chacun pour lui-même. Il faut lire les mémoires de PAsTeur sur l'acide racémique, sur les alcaloïdes des quinquinas 1853), sur l'alcool amylique (1855), sur le sucre du lait (1856) pour connaître les faits sous leur véritable aspect.

$$
\text { *** }
$$

Je n'ose pas affirmer que PASTEur a en l“intuition de la révolution profonde qu'allait produire dans les sciences biologiques la notion de mutabilité des espèces, si bien étudiée depuis 1900 par M. Hugo DE Vries. Mais je ne doute pas $q u$ 'il aurait suivi cette interprétation nouvelle du transformisme avec beaucoup d'intérêt et qu'il aurait trouvé dans ses propres travaux des justifications sérieuses à la théorie qui en découle.

Il faut suivre PASTEur dans sa course à travers l'Allemagne, l'Autriche et jusqu'en Italie, à la recherche de 1 acide racémique, pour avoir une iclée à peu près exacte de 
l'émotion.qui saisit le savant sur la piste de la production d'une espèce nouvelle. En août 1852, PASTEur rencontre Mitscherlich, qui lui apprend qu'en Saxe on obtient encore le fameux corps ; désormais, il n'a plus de repos jusqu'à ce qu'il l'ait tronvé sur place. Or, presque toujours la recherche fut vaine.

L'acide racémique, appelé jusqu'ici paratartrique, fut découvert vers 1820 , par KESTNER, à Thann, comme une altération de l'acide tartrique du Vin; on la supposa particulière au raisin des Vosges ; elle se forma en abondance une seule fois et KestNer en posséda plusieurs centaines de kilogs ; mais dans les nouvelles opérations, elle ne se produisit plus ou se produisit en si faible quantité que la récolte était difficile, sinon irréalisable. Dans les cuves de plomb où l'acide tartrique s'aceumule en gros cristaux transparents, on remarque dans les cavités, et çà et là à la surface, de petites taches blanches soyeuses formées d'aiguilles fines qui constituent l'impureté. PASTEUR découvre que la purifieation des tartres bruts, avant leur expédition aux usines de fabrication d'acide tartrique, explique en partie la rareté du corps, et il montre que les tartres de Hongrie non purifiés en renferment plus que les tartres à demi-raffinés de Naples. Dans les eaux-mères d'une usine de Saint-Maixent (DeuxSèvres), Kestner en trouve une assez forte proportion, plusieurs kilogs, environ le centième en poids de l'acide tartrique traité. L'acide racémique est donc bien une espèce rare, sporadique, qui apparaît comme la fameuse Linaire péloriée de LinNÉ, ȩà et là, en très petit nombre d'exemplaires et, subitement sans cause connue, par masses considérables en de rares stations. Nous avons vu (p. 21) qu'il ne diffère de l'acide tartrique ordinaire que par l'addition en nombres égaux de molécules à dissymétries opposées. 
Pour transformer l'acide tartrique ordinaire en acide. racémique, PAstevr maintient pendant plusieurs heures à une température élevée le tartrate de cinchonine. Il prépare ce dernier sel en dissolvant deux parties de cinchonine dans une solution bouillante renfermant une partie en poids d'acide tartrique; le tartrate de einchonine eristallise dès que la liqueur est refroidie. Chauffé à sec dans un bain d'huile, le composé perd vers 100 degrés son eau de eristallisation; un peu au delà, il entre en fusion. Après deux heures environ à 130 degrés, toute la cinchonine est transformée en une nouvelle base, que PASTEUr désigne sous le nom de cinchonicine. A cette phase, l'acide tartrique est encore intact; une reprise de l'acide montre qu'il est tout entier du type droit. Mais si l'on continue à élever la tem. pérature durant quatre ou cinq heures vers 170 degrés, l'acide tartrique se transforme pour partie en acide racémique. On traite la masse par l'eau bouillante et le chlorure de calcium, et il se dépose du racémate de chaux.

Pasteur s'est assuré, par une série d'éprenves ehimiques et physiques, que le nouvel acide obtenu était en tous points identique à l'acide raeémique naturel, qu'il se dédoublait comme lui en acide tartrique droit et en acide tartrique gauche par quantités égales, possédant des pouvoirs rotatoires égaux et inverses.

Je signale, pour en tirer parti dans les conclusions, une analogie que l'on retrouvera dans les mutations des végétaux [Enothera (DE VRIES), Maïs (Blaringhem)] où se produisent simultanément plusieurs formes nouvelles définitivement indépendantes après leur séparation. PASTEUR a obtenu, au cours de ses recherches, un acide tartrique inactif, " rencontré pour la première fois, mêlé à l'aeide paratartrique, parmi les produits de transformation, sous l'in- 
fluence de la chaleur, des tartrates droits ou gauches (et des paratartrates) de certaines bases organiques. C'est encore ce même acide qui s'est trouvé récemment, et toujours associé à l'acide paratartrique, dans les produits tartriques que MM. PERKin et Duppa ont réussi à préparer à l'aide de l'acide suecinique ". En 1862, date à laquelle PASTEUR signalait ces résultats à la Société chimique de Paris, Dessaignes venait de préparer un acide tartrique inactif qu'il appelait acide nésotartrique; on reconnut qu'il avait les propriétés de l'acide tartrique inactif de PASTEUR. L'identité des formes nouvelles obtenues dans ces diverses transmutations ne peut être le résultat du hasard.

Les expériences de StandFuss (1892-1905) sur les adaptations doubles des chrysalides de Papillons donnant naissance après l'action du chaud $\left(37\right.$ à $\left.39^{\circ}\right)$ ou du froid $\left(4\right.$ à $\left.6^{\circ}\right)$ à des formes locales et à des variations simultanées, ont été exposées plus haut (page 32) comme des phénomènes d'altérations continues et réversibles. Je n'ai pas signalé à cette nccasion, parce que le phénomène est de nature différente, les résultats obtenus par l'action brutale de la chaleur (élévation de la température de 40 à 45 degrés centigrades) ou de la congélation (abaissement de la température de 0 à moins 18 degrés); Standfuss fait d'ailleurs lui-même une distinction entre les deux phénomènes (1). Il constate d'abord, ce qui était à prévoir, que la mortalité dans les conditions brutales est très élevée; de plus, les déviations ne sont plus liées aux types normaux par une série de formes ; enfin, la congélation comme la chaleur donnent naissance

1. - Etudes zoologiques expérimentales sur les Lépidoptères, Annales Soc. Entomol. de France, 1900 , t. LXIX, pp. 82 et suiv. 
aux mêmes types aberrants, formes très rares ou tout à fait nouvelles qui sont en tout comparables à des sports ou mutations. StandFuss s'est efforcé de montrer que ces nouveautés étaient héréditaires et, s'il y avait réussi, ee que certains auteurs eontestent, il aurait établi la possibilité de produire artifieiellement des sous-espèces nouvelles de Papillons.

E. Fischer (1907) paraît avoir donné la preuve décisive

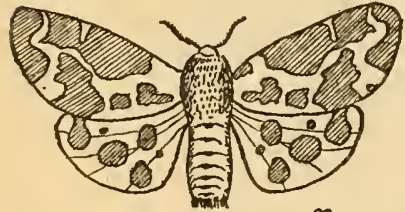

n

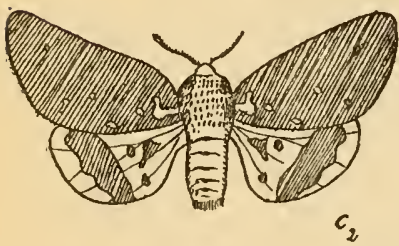

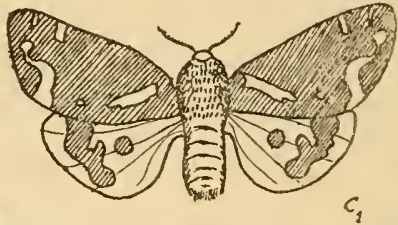

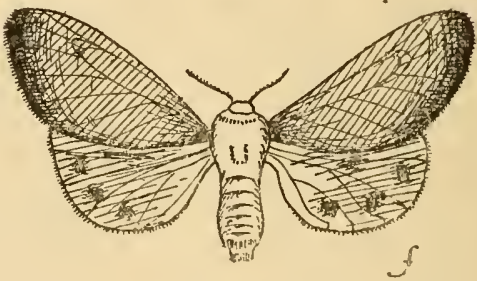

Fig. 14. - Arctia Caja, réduites d'un tiers. - n, papillon normal; $c_{1}, c_{2}$, formes modifiées par la chaleur; $f$, variation commune décrite sous le nom de flavia ( $n$ et $f$, d'après Hofrmann; $c_{1}, c_{2}$, d'après Fischer).

de eette possibilité. Il obtint, après l'action violente de la congélation sur les chrysalides de l'espèce Arctia Caja (fig. $14, n$ ), une coloration brune intense des ailes (fig. 14, $c_{2}$ ) résultant de l'étalement sur la surface des taches brunes qu sont d'ordinaire localisées et sont utilisées pour distinguer les espèces et les sexes. F1scher suivit la descendance d'un couple dont le mâle était beaucoup plus sombre que la femclle, quoique tous deux fussent modifiés dans le même sens. Des œufs obtenus, il réussit l'élevage de 173 descen- 
dants, dont $1 \overline{7}$, soit le dixième, étaient aussi modifiés que les parents (fig. 14, $c_{1}$ ). Les mâles furent ici encore plus méla. niques que les femelles. Depuis cette époque, Kammerer (1913) a réalisé des expériences aboutissant aux mêmes résultats avec des Salamandres, et divers auteurs, PrZibram, surtout SEmon et Rignano, ont utilisé ces résultats pour soutenir l'hypothèse de l'hérédité des caractères acquis.

Il n'y a pas lieu d'insister ici sur les difficultés soulevées par les discussions souvent spécieuses relatives à ce problème, qui est très mal posé, parce que présenté avec toutes les con. séquences qu'on a voulu en déduire à une époque où les notions sur l'hérédité étaient rudimentaires.

Mais, sans quitter le domaine des faits, on peut affirmer que certaines circonstances, très défavorables au développement de l'espèce, entraînent des déviations héréditaires pour partie. En 1905, puis en 1907 , j'ai fourni des preuves décisives des altérations que produisent les traumatismes violents sur la stabilité des caractères des lignées isolées de Maïs (1).

Le Maïs présente deux sortes d'inflorescences; les fleurs mâles sont groupées à l'extrémité des tiges en panicules ramifiées; les fleurs femelles sont condensées sur des épis latéraux, charnus, uniaxes. J'ai montré qu'on peut, à volonté, provoquer la métamorphose des fleurs mâles en fleurs femelles et réciproquement ; puis, par des expériences sériées et répétées durant cinq années sur de nombreuses sortes de Maïs, qu'à un degré de mutilation croissant correspond un pourcentage croissant de grappes anormales,

1. - Action des traumatismes sur la variation et l'hérédité, Thèse, Paris, Alcan, 248 pages et $8 \mathrm{pl}$. doubles. 
qu'à une époque déterminée de la mutilation correspond une intensité déterminée des anomalies. Ainsi, les modifica. tions provoquées, qui présentent toutes les transitions, sont de l'ordre des modifications étudiées dans le deuxième chapitre sous le titre adaptations doubles.

Les graines récoltées sur les panicules anormales du Maïs donnent un fort pourcentage de plantes normales, une
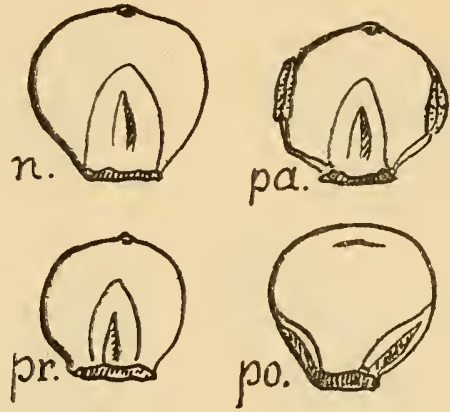

Fig. 15. - Grains de Zea Mays; $n$, grain normal de l'espèce type ; $p r$, grain de l'espèce praecox; pa, grain de la sous-espèce pseudo-androgyna avec deux staminodes latéraux; po, grain de la sous-espece instable polysperma avec deux embryons disposés symétriquement et une attache stigmatique très étalée dans le plan perpendiculaire au plan de symétrie (d'après photographies BlanixgheM).

proportion notable de plantes montrant la métamorphose des épillets mâles en épillets femelles, et quelques plantes qui sont par rapport aux premières des aberrations distinctes, nettement tranchées; ces dernières se répartissent, d'une part, en variétés instables (panachure des feuilles, gaînes des feuilles soudées, port pleureur des hampes), d'autre part, en variétés définies et transmises par hérédité sans aucun retour aux Maïs d'origine lorsqu'on prend soin de les isoler. J'ai obtenu ainsi un Zea Mays praecox (fig. $15 p$ ) à graines mûres en août, alors que le type mûrit 
difficilement en fin septembre (fig. 15, n), un type Zea Mays pseudo androgyna dont les graines sont entourées d'un verticille complet et régulier de trois staminodes (fig. 15, pa). Plus tard, et avec beaucoup de difficultés (1920), j'ai isolé de la même ascendance un Zea Mays var. polysperma, exemple unique de la production dans la famille des Graminées de deux embryons dans la même graine (fig. 15, po). A cette occasion, j'ai insisté sur le parallélisme des altérations observées sur les mêmes plantes dans les épillets femelles et dans les épillets mâles, dont les fleurs ofirent 4, 5, 6 étamines au lieu de 3 (1).

Or, ma conviction a été, dès le début, que les circonstances particulières, d'ordre physique, dans lesquelles se trouvent les éléments sexuels des plantes mutilées sont la cause immédiate de ces métamorphoses et de ces aberrations : "Les déviations morphologiques sont la conséquence de conditions physiologiques anormales déterminées par la rupture d'équilibre des fonctions. L'époque la plus favorable à la production d'anomalies végétales par des mutilations est celle du maximum de croissance de l'individu soumis an traumatisme. Il existe alors une opposition violente entre l'absorp. tion de l'eau par les racines et la diminution brusque de la transpiration; l'excès d'eau modifie les jeunes tissus des bourgeons adventifs et détermine les troubles graves dans la formation des organes dont le retentissement se produit jusqu'aux éléments sexuels et, par là, à la postérité.....

"Les variations héréditaires provoquées par des traumatismes affectent tous les caractères de l'espèce. Il en résulte une pulvérisation du type en des formes multiples et dis-

1. - Production par traumatisme d'une forme nouvelle de Maïs à caryopses multiples Zea Mays var. polysperma, $-C$. R. Acad. Sciences, 15 mars 1920, t. CLXX, - p. 677. 
tinctes, qui sont tantôt de nature régressive et rappellent les ancêtres du Maïs (1), tantôt de nature progressive et montrent l'acquisition par l'espèce Maïs de caractères nouveaux pour le genre, la tribu et même la famille des Graminées. » (Thèse, 1907, pp. 225-226.)

Quelle est la nature des nouvelles formes obtenues ? Il ne peut $\mathrm{y}$ avoir aucun doute qu'elles doivent être classées dans le genre Zea et même dans l'espèce Zea Mays; ce sont des déviations inattendues, mais non incompatibles avec les principales caractéristiques du genre, et du fait qu'elles n'ont pas été jusqu ici décrites, ni peut-être rencontrées dans les vastes cultures des plantes de cette espèce, il n'en résulte pas qu'il faille donner aux particularités nouvelles une importance que la Systématique seule leur accorderait. La Systématique est un groupement artificiel dont les règles doivent être modifiées avec les circonstances et avec les progrès de la découverte des affinités.

Il résulte des études si approfondies de M. DE VRIES sur les dérivés de l'Enothera Lamarckiana, de celles de M. T.-H. Morgan sur les mutations de la petite Mouche du Vin Drosophyla ampelophila, des aberrations provoquées par STANDFuss, Fischer et d'autres sur les Papillons, de mes observations sur le Maïs, que le groupement spécifique n'est pas

1. - J'ai été conduit à interpréter certaines des déformations héréditaires sériées obtenues dans mes essais, comme une preuve de la dérivation du genre $Z e a$, à partir de fasciations fixées du genre sauvage amérieain Euchlaena mexicana, et j'ai indiqué la série des expériences à faire, en climat tropical, pour démontrer cette liaison. Le 2 octobre 1922, M. José DE CAMpos-Novaes, directeur de l'Institut agronomique de Campinas, Sao-Paulo (Brazil), m'écrit avoir réussi cette métamorphose après cinq ans de sélec_ tion d'anomalies, et les photographies que son collaborateur, M. Bento DE Toledo, m'adresse sont tout à fait probantes. 
ébranlé, mais que le polymorphisme y est plus aceusé qu'on ne pouvait le prévoir d'après le reeensement des formes connues. Les jardiniers, qui opèrent d'ordinaire avee des variétés mal définies, traduisent les tendanees multiples que mettent en évidenee les hybridations et les mutations par un terme qui traduit assez correctement le phénomène : l'espèce est en état d'" affollement ", mais elle n'en reste pas moins l'espèee.

Il est probable que l'état de mutation met en évidence chez les êtres vivants le eomplexe de eombinaisons et d'états d'équilibres possibles par l'union de plusieurs groupements dissymétriques, qui présentent des affinités réelles sans quoi l'association serait impossible. Dans une conférence faite à la Société chimique de Paris (1), Pasteur expose diverses eonsidérations qui me paraissent devoir s'appliquer à la mutation et aux cireonstances de déséquilibre qui l'accompagnent.

Tous les tartrates droits de bases inaetives ont leurs dissymétriques inverses dans les tartrates gauches. On sait que Pasteur en déduit la possibilité de posséder quatre corps de même composition chimique, mais d'arrangements moléeulaires différents, le droit, le gauche, l'union du droit et du gauche et l'inactif.

Combinons l'aeide tartrique avec l'aeide malique, ou plutôt le tartrate d'ammoniaque avee le malate d'ammoniaque, dissymétrique comme le tartrate; ce tartromalate aura une forme telle qu'elle en comportera trois autres semblables, mais ces quatre formes élémentaires ne seront pas superposables les unes aux autres, e'est la tétartoédrie... "Supposons, continue Pasteur, que j'unisse l'aeide tartrique

1. - Revue Scientifique, 1884, t. VII, pp. 2, 6. 
à l'acide malique et à la quinine (à la morphine, à la cinchonine...), j'aurai trois groupes actifs réunis. Or, la forme cristalline de l'assemblage en comportera sept autres pareils non superposables. $\mathrm{Au}$ lieu de trois groupes dissymétriques rérunis ensemble, prenez-en quatre et en les combinant avee leurs inverses, quatre à quatre, vous aurez seize assemblages possibles et, par conséquent, chaque forme en comporterait quinze autres pareilles. - La combinaison de cinq groupes dissymétriques comporterait trente-deux assemblages, et ainsi de suite. " L'addition des droits et des gauches, l'existence prévue de l'inactif ne résultant pas de cette addition, élève considérablement le nombre des pasitions d'équilibre moléculaires de ces combinaisons ehimiques définies.

" Eh bien, Messieurs, ajoute PASTeur, il y a ici une impossibilité cristallographique. La dissymétrie simple correspond à une dissymétric inverse. Au droit répond le gauche. Une dissymétrie double peut donner lieu à quatre combinaisons. Dans le premier cas, c'est l'hémiédrie ; dans l'autre cas, c'est la tétartoédrie ; mais étant données les lois de la eristallographie, il n'est pas possible d'imaginer l'octoédrie ; ce mot même n'a jamais été employé, et encore moins a-t-on imaginé toutes les dissymétries plus élevées suivantes. Les plus habiles cristallographes, praticiens ou mathématiciens, je le répète, non seulement n'ont pas rencontré, mais encore n'ont pas supposé l'existence possible de l'octoédrie.

"Ici se présente une des propositions, à mon avis, les plus curieuses. Vous savez que les molécules les plus complexes de la chimie végétale sont les albumines. Vous savez, en outre, que ces prineipes immédiats n'ont jamais été obtenus à l'état cristallin. Ne peut-on ajouter que, vraisemblablement, ils ne peuvent pas cristalliser? Pour comprendre 
l'impossibilité de leur eristallisation, d'après ce qui vient d'être dit, il suffit d'imaginer qu'ils sont constitués par trois groupes moléculaires dissymétriques, à plus forte raison, s'ils l'étaient par quatre, par cinq, etc., etc... En d'autres termes, pour faire les produits essentiels de la vie, les principes immédiats de nos tissus, de notre sang, prineipes qui doivent être mous, flexibles, glissants, non cristallins, la nature, pour faire ces produits de la vie, n'aurait qu'à unir un nombre minimum de trois groupes dissymétriques. "

J'ajouterai, pour en finir avec la mutation, que si les organismes spécifiques sont le résultat de la combinaison de trois corps dissymétriques au moins, les formes possibles principales sont au nombre de huit, et il n'y a pas lieu de s'étonner que lorsqu'une espèce est ébranlée, soit par le milicu, soit par l'hybridation, il résulte de cet ébranlement non pas une seule forme d'équilibre nouvelle, mais plusieurs. Autrement dit, l'épreuve expérimentale le vérifie lorsqu'il s'agit réellement de changements spécifiques, la mutation est une altération par explosion avec des résultantes dans diverses directions, indépendantes et immédiatement fixées dans leur nouvel état d'équilibre; c'est la pulvérisation de l'espèce et non le transformisme au sens habituel du mot. 



\title{
LIVRE II
}

\section{LES GERMES}

\author{
CHAPITRE IV
}

\section{LE ROLE DES GERMES}

Dans la même Conférence (1884), Pasteur donne aussi son opinion sur les germes, et ses aphorismes prennent un caractère si profond qu'il faut laisser à chacun le soin de les méditer.

" On trouve la dissymétrie établie dans un très grand nombre de principes immédiats des animaux et des végétaux, notamment dans les principes immédiats nécessaires à la vie. Tous les produits, pour ainsi dire, de l'œuf et de la graine sont dissymétriques. ))

Laissons de côté l'urée, l'acide oxalique, produits de seconde main, déchets de la vie ; ils sont comme les produits de synthèse chimique chez lesquels la dissymétrie est absente... M. Lorr trouve que la nitromannite est active ; sur la demande de Pasteur, Bichat étudie la mannite et reconnaît qu'elle n'est pas inactive comme on le croyait auparavant. Lorsque Lebel a voulu extraire de ses paratartrates de synthèse des actifs simples, il a eu recours à 
l'emploi d'un dissymétrique, ou d'une moisissure ou d'un microbe ; l'intervention de microbes, de petits champignons mieroscopiques, voilà le moyen le plus simple, découvert par PASTEUR, pour faire apparaître la dissymétrie : " J'ai fait vivre, dit PASTeur, de petites graines (spores) de penicillium glaucum, de cette moisissure qu'on trouve partout, à la surface de cendres et d'acide paratartrique, et j'ai vu l'acide tartrique gauche apparaître. C'est encore la dissymétrie simple obtenue avec un corps inactif ; mais toujours également, pour arriver à ce résultat, il a fallu, vous le voyez, faire intervenir des actions de dissymétrie, la dissymétrie des produits immédiats naturels qui composent la graine de la moisissure...

" Ces expériences accusent une ligne de démarcation profonde entre le règne minéral et le règne organique, puisque, pour imiter ce que fait la nature, c'est-à-dire préparer un corps droit ou un corps gauche, nous sommes contraints de faire intervenir des actions particulières, des actions de dissymétrie... Je pressens même que toutes les espèces vivantes sont primordialement, dans leur structure, dans leurs formes extérieures, des fonctions de la dissymétrie cosmique. La vie, c'est le germe, et le germe, e'est la vie. ")

A cette date, PAsteur avait triomphé de toutes les objections, de toutes les critiques que lui avaient opposées les partisans de la génération spontanée. Il était dans l'éclat du triomphe que lui avaient aequis ses méthodes de stérilisation, sa technique d'atténuation des virus et de la préparation des vaccins. Avec une maîtrise qui résulte de la parfaite confirmation de ses déductions logiques, il déclare: "Qui pourrait dire ce que seraient les " devenir 》 des germes si l'on pouvait remplacer dans ces germes les principes immédiats, albumine, cellulose, etc., etc., par leurs dissymétriques 
inver'ses ? La solution consisterait, d'une part, dans la découverte de la génération spontanée, si tant est qu'elle soit en notre pouvoir ; d'autre part, dans la formation de produits dissymétriques à l'aide des éléments carbone, hydrogène, azote, soufre, phosphore, si, dans leurs mouvements, ces corps simples pouvaient être dominés, au moment de leur combinaison, par les forces dissymétriques. " Le problème de l'origine de la vie est nettement posé ; peu d'efforts ont été tentés pour le résoudre ; qui reprendra l'étude de ces questions primordiales?

En 1856, Pasteur découvrit l'intervention des microbes dans la disparition de la dissymétrie, par la fermentation du sucre du lait; il avait fait auparavant une étude de l'alcool amylique (1855), mélange variable de corps actifs et de corps inactifs, qu'il réussit à séparer avec le sulfate de baryte. La spécificité des germes s'est-elle imposée dès cette époque à son esprit ? Je n'en ai pas trouvé de preuves décisives, et je suis tenté, pour la clarté de la démonstration, de faire un saut de dix années et d'emprunter à GenNez, alors préparateur de PASTEUR à l'Ecole normale, le fil qui me guidera dans l'analyse des conceptions du maître. Je présume, en effet, ‘u'il y a dans l'évolution des conceptions de PAsteur deux phases : la première où il étudie l'essence spécifique, ses variations, sa continuité dans les composés ; la seconde, au cours de laquelle il définit l'individu, la culture pure, les modifications dues au mode de vie cui donnent les ferments figurés et les vaccins. J'ai divisé le résumé de cet ouvrage en deux parties pour mettre en valeur cette distinction. Il ne faut pas oublier, d'autre part, que PASTEUR a été entraîné, par l'importance pratique des conséquences de ses découvertes, à faire l'étude complète des fermentations, à découvrir une méthode générale de préparation des 
vaceins sans perdre à aucun moment l'espoir de préeiser ses conceptions sur les phénomènes de la dissymétrie moléculaire ; M. PASTEUR VALLERY-RADOT, en faisant connaître le projet de publication, en 1878, d'un ouvrage d'ensemble sur ce sujet, nous en donne une preuve certaine.

Gernez, agrégé de Physique, fut, dès le début de ses études, entraîné dans la voie brillante ouverte par PASTEUR, et sa thèse de doctorat, qu'il prépara avee une habileté expérimentale consommée et une patience caractéristique de son tempérament, fut, en quelque sorte, la continuation des travaux de Pasteur sur les phénomènes optiques de la dissymétrie moléculaire. Elle parut dans les Annales scientifiques de l'Ecole Normale en 1864, sous le titre Recherches sur le pouvoir rotatoire des liquides actifs et de leurs vapeurs. PASTEur retint Gernez à son laboratoire; il l'entraîna à Alais en 1865, et lui confia une part importante des études sur la Pébrine du Ver à soie ; durant des années, le maître et l'élève furent en rapports presque journaliers ; nous suivrons PASTEUR en analysant les travaux de GERnEz.

Le 12 novembre 1886, Pasteur lut, à l'Aeadémie des Sciences, l'extrait suivant d'une lettre que lui adressait Gernez :

" J'ai reconnu qu'une solution sursaturée de tartrate double de soude et d'ammoniaque gauche ne cristallise pas au eontact d'un fragment du même sel hémièdre à droite et vice versa, la solution saturée du sel droit n'abandonne pas de cristaux quand on la touche avec le sel gauche.

"Ce fait m'a conduit à étudier la solution inactive de racémate double de soude et d'ammoniaque. Avec I'acide racémique que vous avez obligeamment mis à ma disposition, j'ai fait une solution saturée de ce sel. Touchée par une par- 
celle de sel droit, elle n'a abandonné que des cristaux droits; une portion du même liquide, au contact d'un cristal gauche, a produit un dépôt de sel gauche. Voilà done un moyen simple de séparer à volonté du racémate double de soude et d'ammoniaque en l'un ou l'autre des deux sels qui le constituent. »

J'ai esquissé, plus haut, en traits rapides, l'histoire de ce fameux acide racémique, qui joua dans la carrière et dans les conceptions géniales de PASTEUR un rôle si important. Nous avons vin que la constatation de l'inactivité des solutions de ce corps, qui a la même composition chimique que l'acide tartrique ordinaire, a conduit PASTEUr à la notion de la dissymétrie moléculaire (p. 21), puis à la découverte d'une méthode de transmutation d'une sous-espèce en une autre, par les actions simultanées de la combinaison de l'acide tartrique actif avec une base active, et de la chaleur élevée qui facilite la désintégration moléculaire (p. 75). En 1858, Pasteur fait connaître un autre procédé de séparation des sous-espèces combinées dans l'acide racémique, qui repose sur les actions limitées des germes.

L'acide tartrique fermente spontanément. PASTEUR ajoute à une solution de tartrate ordinaire d'ammoniaque pur des traces de matières albuminoïdes mortes et, après l'ébullition, la température étant descendue à $30^{\circ}$ environ, quelques centimètres cubes du liquide trouble d'une bonne fermentation en train de tartrate ordinaire commercial. Le microscope permet d'y décéler des microorganismes; leur action sur le tartrate d'ammoniaque est immédiate, et bientôt la solution devient inactive par destruction de l'acide tartrique. Le racémate d'ammoniaque fut mis en fermentation dans les mêmes conditions et elle se déclara avec la même facilité. Mais ici l'appareil de polarisation montra que le liquide, 
d'abord inactif, possédait après quelques jour's un pouvoir rotatoire à gauche sensible, et ce pouvoir angmenta progressivement jusqu'à un maximum. "La fermentation est alors suspendne. Il n'y a plus trace d'acide droit dans la liqueur, qui, évaporée et mêlée à son volume d'alcool, donne immédiatement une abondante cristallisation de tartrate gauche d'ammoniaque. Voilà, sans doute, un excellent moyen de préparer l'acide tartrique gauche » (1).

Les conséquences de cette nouvelle méthode, physiologique, de séparation de l'acide tartrique droit et de l'acide tartrique gauche dans le complexe acide racémique, furent immédiatement ramenées par PAsteur à l'intervention des dissymétries propres au ferment, détruisant, dans la nature, l'acide tartrique ordinaire. Il y trouva, à la fois, une confirmation des conceptions exposées plus haut, dont l'expression ne s'est précisée qu'avec le temps, mais qui dominaient certainement déjà l'enchaînement logique des faits observés, et un point de départ pour la justification des procédés de culture des microorganismes, si sensibles à l'état moléculaire des substances minérales mises à leur disposition, qu'ils n'attaquent dans le complexe que la partie donée d'une certaine dissymétrie moléculaire et laissent intact le corps identique par ses éléments chimiques mais différant par l'arrangement de ces éléments dans la molécule.

Gernez découvre un troisième procédé de désagrégation de l'acide racémique, très simple, puisqu'il ne fait intervenir qu'un fragment de cristal de l'un on de l'autre acide, instantané puisque le dépôt par cristallisation ne demande que quelques heures, et surtont logique, car il est l'application

1. - Mémoire sur la fermentation de l'acide tartrique, C. R. Acad. Sciences, t. XLVI, p. 615. 
d'une méthode courante en cristallographie. Il ne fait intervenir ni les combinaisons de corps divers qui laissent toujours planer un doute sur la nature même de ces combinaisons, ni les ferments dont il faut reconnaitre les propriétés sans pouvoir en expliquer le mécanisme. Avec la sûreté du génie, Pasteur accepta de son élève déférent le procédé, et de suite, le fait connaitre au mondre scientifique comme une découverte de premier ordre.

Les naturalistes qui examineront la découverte de GERNEZ ne manqueront pas de comparer l'élément de cristal, qui transforme à son image une partie du mélange sursaturé, à un germe qui utilise une nourriture appropriée, mise à sa portée, pour croître et acquérir les dimensions physiques limites ; mais Gernez est physicien et, à mon avis, dans cette association de savants, élucidant les problèmes de la eristallisation et de la dissymétrie, c'est PASTEUR qui a dû avoir, avec force, l'intuition qu'il $y$ avait dans l'expérience de GeRnEz beancoup plus qu'un procédé commode pour séparer des espèces moléculairement distinctes dans un mélange intime.

Elle était, d'ailleurs, la conséquence naturelle d'une série de travaux sur la cristallisation des substances sursaturées, dont Gernez avait présenté les principaux résultats à l'Académie des Sciences en 1865, et dans un Mémoire publié dans les Annales scientifiques de l'Ecole Normale, la même année. Elle touche de trop près à la génération spontanée pour qu'il soit superflu d'en faire un exposé rapide.

En 1812, H. DAvy signale qu'une solntion chaude de sulfate de soude, privée d'air par la machine pneumatique, se refroidit sans cristalliser, et, par conséquent, reste, si la concentration est suffisante, en état prolongé de sursaturation ; 
la rentrée brusque de l'air dans le vase clos y détermine une eristallisation presque instantanée. GAY-Lussac répète l'expérience, la vérifie, eonstate que la eristallisation n'a pas toujours lieu et, qu'en tont eas, la protection de la solution chaude par une couche d'huile maintient la solution liquide indéfiniment, même si l'air est à la pression normale. En 1814, Schweiger signale que la simple proteetion par une feuille de papier de l'ouverture du boeal où la solution de sulfate de soude a été chauffée suffit pour maintenir la solution en état de sursaturation; que eet état cesse si l'on introduit, après refroidissement de la solution, un eorps quelconque, et, avec plus de sûreté, un fragment solide de sulfate de soude. En 1815, Ziz affirme que les corps étrangers (tige de verre ou de fer) perdent toute action si on a soin de les laisser séjourner quelques instants dans l'eau ou de les ehauffer dans la flamme d'une lampe à alcool.

La question reste en suspens durant une trentaine d'années, malgré des études soignées de Grahan, Odgen, qui confirment les résaltats préeédemment établis. En 1852, Lawew découvre que l'air filtré sur du eoton, barbottant par aspiration dans la solution sursaturée, n'en détermine pas la eristallisation, qui est eonstante lorsque l'air aspiré est pris dans la même atmosphère du laboratoire, mais non filtré. Il suppose que la filtration sur eoton a fait perdre à l'air son pouvoir eatalytique; il laisse donc le mystère entier. Mais il n'est pas inutile de faire remarquer l'emploi du eoton comme protecteur contre les germes et l'attribution à Schrgeder et Dusch de la première utilisation du coton pour arrêter les miasmes de l'air est ineorrecte, si on veut bien admettre que le mot miasme n'avait, à cette époque (1854), aucune signification précise. En 1858, ScIIrcEDer, interprétant l'expérience même de LEwEL, prétend prouver 
que la cristallisation est produite par des miasmes de la même nature que ceux qui déterminent la fermentation et la putréfáction.

Ces différentes étapes de l'étude des conditions des solutions sursaturées n'avaient pu échapper à l'attention de Pasteur, à l'aftût de tout ce qui concernait la cristallographie et les fermentations ; e'est pourquoi j'ai affirmé plus haut que PASteur et Gernez devaient être associés dans la découverte du germe eristallin provoquant, par sa seule action, le changement d'état physique des solutions sursaturées.

M. Violette, normalien conscrit de PASTEUR (promotion de 1846), ancien de GERNEz (promotion de 1855), étudiait vers cette époque les mêmes problèmes, comme le prouve la note intitulée : Recherches sur les causes de la cristallisation des solutions sursaturées, qu'il présenta à l'examen de l'Aca. démie des Sciences en 1860. D'après lui, Schrcder compliquait la question en s'assurant que l'addition de corps calcinés, tels que la chaux et la baryte, tous deux avides d'eau, provoquaient la cristallisation, malgré leur traitement par la flamme. Bref, en 1865, simultanément et à l'insu l'un de l'autre, Violette à Lille et Gernez à Paris démontraient :

$1^{\circ}$ Que la cristallisation des solutions sursaturées de sulfate de soude devait être attribuée au contact d'une parcelle solide de cette substance; $2^{\circ} q u$ 'il y a en suspension dans l'atmosphère des parcelles de sulfate de soude.

Le plus curieux, dans cette concordance des conclusions, e'est que PASTEUR s'était rendu compte que l'un et l'autre de ses élèves étaient arrivés au même résultat. Lorsque Gernez lui présenta son travail, Pasteur l'avertit qu'il convoquerait M. VIOLETTE pour lui demander quel était son avis sur la question. 
Des coïncidences aussi parfaites avec les conclusions que Pasteur présentait, à la même date, relatives à la génération spontanée, devaient singulièrement renforcer la certitude qu'il avait acquise, par des séries d'autres expériences, que l'introductiou des germes était nécessaire et suffisante pour déterminer les fermentations et les putréfactions.

En donmant une revue d'ensemble de la question (1), soutenu par cette notion des germes que Pasteur démontrait si efficaces dans les altérations des solutions minérales et organiques, GERnEz montre donc, avec une méthode impeccable, qu'il n'y a qu'une seule méthode, infaillible, pour faire cesser l'état de sursaturation, c'est le contact d'une parcelle, si minime soit-elle, de la substance solide qui doit se former par la cristallisation.

Il s'assure, et c'est le point essentiel de sa démonstration, que le sulfate de soude renfermant dix molécules d'eau est nécessaire pour entrâner la cristallisation ; le sulfate auhy. dre reste sans action; or, le premier se transforme en le second par un simple flambage; soumis à la température de $33^{\circ}$, le sulfate actif perd son eau de cristallisation et devient inactif. Le flambage des vases, des agitatemrs, des papiers protecteurs, devient l'essentiel de la technique de contrôle, et désormais, il n'y a plus d'insuccès; l'introduction seule du cristal germe suffisamment hydraté, mais solide, réussit; en son absence, l'agitation du liquide, qui donme si souvent lieu à des cristallisations pour ainsi dire spontanées, reste sans effet.

La vérification est poussée à l'extrême ; tous les produits

1. - Sur les solutions sursaturées, Bulletin de la Societé Chi. mique, t. VIII, 1867 , pp. 153 et suiv. 
du laboratoire de l'Eeole Normale, au nombre de 276, sont successivement employés pour mettre en évidence leur activité sur la solution de sulfate de soude sursaturée ; 39 sont actifs. Parmi eux, 19 sont insolubles dans l'eau ; GERNEz les lave, puis les dessèche à l'abri de l'air, et tous perdent leur activité ; ils la devaient incontestablement aux poussières superficielles de sulfate de soude cristallisé qui les recou. vraient. Les vingt autres, solubles, sont purifiés par plusieurs cristallisations à l'abri de l'air ; tous, sauf un, perdent leur pouvoir de déterminer la cristallisation; l'unique corps actif est le sulfate de soude lui-même, cristallisé avec dix molécules d'eau.

Enfin, il faut prouver qu'il y a des parcelles de sulfate de soude en suspension dans l'atmosphère du laboratoire. Faisant barbotter 2.000 litres d'air dans quelques centimètres cubes d'eau, Gernez met le sulfate de soude en évidence par les réactions chimiques habituelles.

La solution d'acétate de soude est, elle aussi, mise à l'épreuve; l'état de sursaturation de l'acétate se maintient beaucoup plus longtemps sans précautions spéciales. GERNEZ vérifie que des filtrations de l'air dans l'eau établissent rarement la présence d'acétate en suspension dans l'atmosphère; il découvre aussi que les cristaux ne deviennent inactifs qu'à $79^{\circ}$. Voilà done un moyen délicat pour mettre à la fois en évidence l'existence et les propriétés des germes spécifiques.

La découverte de la sexualité chez les végétaux est illus. trée d'exemples analogues dont la répétition, complétée par des expériences décisives seulement au milieu du xıx siècle, entraîna la conviction de la généralité du phénomène, exception faite pour certains Figuiers, Bananiers et Kakis partlénocarpes. 
Préeisément, quelques exceptions rendaient douteuse la nécessité d'un pollen approprié pour la formation de fruits et de graines. De L'EcLuse rapporte, dès la fin du Xvi ${ }^{\mathrm{e}}$ siècle, que le Papayer (Carica Papaya) a deux sortes d'individus à fleurs diftérentes et désigne celui qui porte des étamines, mâle, l'autre, pourvu d'ovaires, femelle: "On prétend, ajoute-t-il, que des affinités mystérieuses mnissent l'un à l'autre les végétaux en question, de telle sorte que la plante femelle ne porte pas de fruits lorsque la plante mâle est séparée d'elle par un espace étendu, au lieu de se tronver à proximité. " Camerarius, au début du xvinI ${ }^{e}$ siècle, prit partı pour la sexualité et la fécondation nécessaires à la formation des graines ; ses épreuves sur les Maïs castrés, sur les pieds de Chanvre femelle isolés dans son jardin, ne lui donnèrent pas eonstamment des insuccès ; dans quelques cas, il récolta des graines peu nombreuses, mais féeondes, dont il ne sut expliquer la production ; il ignorait le transport du pollen par le vent à de longues distances.

Gleditscir publia, en 1751, me expérience qu'on aurait pu eroire décisive. Un Palmier femelle de quatre-vingts ans existait au jardin botanique de Berlin, et le jardinier assurait n'avoir jamais observé de fruits; GLEDITSCH fit venir de Leipzig une infloreseence mâle de la même espèce; le voyage dura neuf jours et, dans le transport, la majeure partie du pollen était tombée ; il lia cependant cette infloreseence à l'inflorescence presque flétrie de l'arbre du jardin botanique de Berlin ; l'hiver suivant, il obtint des fruits qui germaient au printemps de l'année 1750 .

Malgré ces faits probants, la eonviction que la sexualité végétale était en tous points comparable à la sexualité des animaux ne fut définitivement imposée qu'après les remarquables expériences de Tiruret (1845) avec les Fucus. Dans 
une cuvette d'eau de mer, Thuret dilacère les réceptacles ovoïdes d'un certain pied de Fucus vesiculosus; dans une autre cuvette, ceux de formes plus arrondies, plus charnues, d'un autre pied de la même espèce qui, on le sait depuis, renferme préeisément des individus mâles et des individus femelles. Dans le premier bocal, si le pied mâle est à point, le liquide prend une teinte orangée, et le microscope y montre des organites mobiles très petits, à deux cils (fig. 16,a) ;

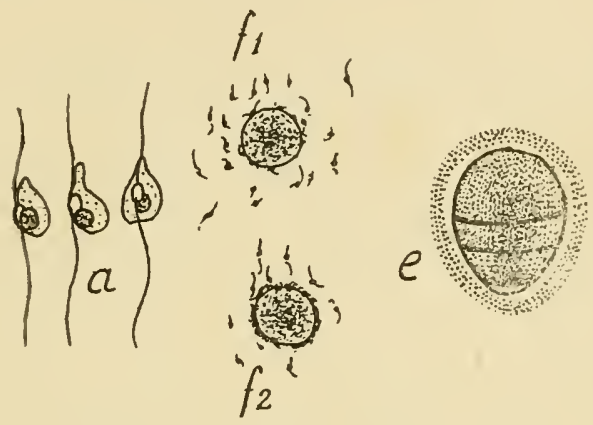

Fig. 16. - Fécondation du Fucus daprès Tuunet ; a, anthérozoïdes mobiles très grossis: $f_{\mathrm{i}}$, première phase de la féeondation, l'ovule, entouré d'anthérozuïdes, tourne surlui-même: $f_{2}$, un ant!iérozoïcle au moins a pínétré; la membrane sépaissit, l'œuf devient immobile; e, embryon en voie de division.

laissés à eux-mêmes, ils meurent au bout de quelques jours. Dans l'antre bocal, les réceptacles ont abandonné des cellules rondes, grosses, qu'il faut cependant examiner au microscope pour définir leurs contours ; elles donnent au liquide une teinte olivâtre et sont immobiles ; elles se déposent et meurent aussi au bout de quelques jours. Thuret mélange les deux liquides ; il en examine une goutte sous le microscope et constate qu'un certain nombre des organites mobiles se groupent à la surface des sphères olivâtres (fig. 16, $f_{1}$ ), 
que ces organites s'appliquent sur elles, leur impriment, grâce aux mouvements de leurs cils, une rapide rotation, qui cesse au bout d'une demi-heure environ; après une légère contraction du contenu (fig. 16, $f_{g}$ ), il se forme une solide membrane de cellulose ; c'est l'œuf, qui ne tarde pas à se cloisonner (fig. 16,e). Les divisions n'ont lieu qu'après le mélange des liquides; la fécondation s'est produite par un processus déjà connu chez la plupart des animaux ; il y a donc des éléments sexuels équivalents dans les deux règnes.

En réalité, on soupçounait bien qu il suffisait de l'entrée d'un seul organite mobile dans la sphère inerte pour produire un œuf ; mais la démonstration est délicate, et il fallut de nombreuses vérifications méticuleuses pour que la certitude fût acquise définitivement. Une expérience de CharLess NAUdin (1863), complétant des épreuves analogues de KøLREUTER (1765), a prouvé qu'un seul grain de pollen suffit pour déterminer le gonflement des fruits et la production d'une graine saine dans le genre Mirabilis; les plantes obtenues des graines résultant d un seul pollen sont rarement aussi vigoureuses que celles qui dérivent des graines ordinaires ; mais la question qui nous occupe est clairement élucidée : un germe unique, ici un grain de pollen, suffit pour donner naissance à un individu nouveau.

Les conséquences générales des expériences de Thuret, de Naudin, n'ont pu échapper à PaSteul et à ses élèves qui, à cette époque, imaginaient et perfectionnaient les méthodes de culture pure des ferments figurés et des microbes. J'ai roulu les rappeler pour affirmer les analogies qui apparaissent dans les eristallisations des solutions sursaturées et dans les fécondations croisées, par le vent ou par les insectes, pour en tirer parti daus mes conclusions. 


\section{$\stackrel{* *}{* *}$}

Il serait dangereux de suivre plus loin les analogies qui peuvent être mises en évidence entre les formes cristallines et les êtres vivants sans prendre quelques précautions. L'état cristallin, les phénomènes de dimorphisme, la dissymétrie moléculaire, étudiés par PASTEUR, sont l'exception dans les substances minérales ; l'existence même de ces particularités exceptionnelles fait découvrir en quelque sorte les liaisons et démontre la persistance, à travers les divers états physiques, des qualités particulières de la structure propre des molécules. L'état de sursaturation est, lui aussi, une circonstance exceptionnelle que la plupart des corps ordinaires ne possèdent pas ; l'alun, le carbonate de soude, l'acétate de sonde, le sulfate de magnésie, le sulfate de zine, etc..., en général tous les sels dont les cristaux retiennent une grande proportion d'eau présentent des tendances plus ou moins marquées, spécifiques, à la sursaturation ; les sels anhydres ne la présentent pas et leurs dissolnations, mises à l'abri de l'air, cristallisent exactement comme si elles étaient à découvert.

Il n'y a done pas lieu de s'attarder aux critiques présentées dès la publication des découvertes de Violetrte et de Gernez : un monde de germes cristallins de toute espèce, en suspension dans l'air et se déposant sur les agitateurs des laboratoires, pouvait seul justifier pour certains les phénomènes de cristallisation des solutions sursaturées observés un peu partout. L'extension illégitime d'une expérience parfaitement exécutée fut évidemment présentée pour détruire par l'absurde les conséquences, alor's très discutées, de l'absence de génération spontanée soutenue par PASTEUR. On a voulu de même faire de l'absence de génération spon- 
tanée un cas exceptionnel, alors que Pasteur était convaineu de sa généralité en l'état actuel de la question et l'affirmait courageusement.

Je ne crois pas que PASTEUR ait pris cette attitude sans avoir longuement réfléchi, et surtout sans avoir cherché à faire apparaître, par divers procédés expérimentaux, ces fameuses propriétés de déviation de la lumière polarisée des solutions ou de l'hémiédrie cristalline non superposable, dont il fait remonter l'origine à la structure même de la molécule. En fait, Gernez a réussi dans quelques cas à transformer une solution de cristaux droits en cristaux gauches, et inversement; ces exemples de métamorphose artificielle des qualités les plus intimes des espèces cristallines méritent d'être analysées brièvement; nous allons voir qu'ils sont du type des variétés et non du type des sousespèces.

Dans une communication de Gernez, présentée par PasTEUr à l'Académie des Sciences en 1868 (1), on trouve un exemple précis de ces tentatives. Fn 1850, PAsTEur avait signalé le cas spécial du formiate de strontiane et recomm, par l'examen des cristallisations de ce sel, qu'il y a toujours deux espèces de cristaux, les uns hémièdres à droite, les autres hémièdres à gauche, identiques par conséquent, mais non superposables. En séparant à la main, comme dans les sels de l'acide racérnique, les eristaux droits et les cristaux gauches, en faisant ensuite leurs solutions, il avait été frappé par la circonstance inattendue que ni l'une ni l'autre des dissolutions n'agissait sur la lumière polarisée. "Ceci

1. - Sur la cristallisation des substances hémiédriques, $C$. $R$. Ac. Sc., t. LXVI, p. 353. 
conduit à supposer, dit PASTEUR, que l'hémiédrie du formiate de strontiane ne tient pas à l'arrangement des atomes dans la molécule chimique, mais à l'arrangement des molécules physiques dans le cristal total..... ; quaud on fait cristalliser de nouveau des cristaux droits ou des cristaux gauches de formiate de strontiane, chaque espèce unique fournit les deux espèces de cristaux. " C'est en quelque sorte une forme d'adaptation double, indépendante de la molécule.

Gernez (1868) examine les circonstances qui font cesser la sursaturation des solutions relativement peu concentrées de formiate de strontiane ; elles se conservent limpides à la température ordinaire, même dans le laboratoire, où il y a certainement des particules de ce sel plus ou moins dispersées, si l'on prend soin d'incliner le col du ballon renfermant la solution pour empêcher ces particules de tomber dans la solution sursaturée.

A l'aide d'une tige métallique propre, Gernez y introduit un eristal droit qui détermine une prise et bientôt le dépôt d'une masse considérable de cristaux, tous droits ; au cours de vingt opérations, on n'obtient ainsi aucun cristal gauche ; " la parcelle cristallisée (germe) détermine done l'orientation de toutes les molécules qui se déposent successirement les unes sur les autres $)$.

Gernez va plus loin : il sépare l'eaul-mère de la première cristallisation, en prenant toujours les précautions néces. saires pour éviter la cristallisation spontanée ; il y ajoute de nouveau un cristal droit, une nouvelle prise s'y produit, et tous les cristaux obtenus sont encore uniquement des cristaux droits. Continuant l'expérience, par cristallisations avec germes droits et décantations successives des eaux-mères, il épuise la masse dissoute et la transforme tout entière en cristaux droits. 
L'inverse est possible ; en redissolvant les eristaux obtemus et ensemençant la solution et les déeantations suceessives avec des cristaux gauehes, la masse eristalline résultante est uniquement composée de cristaux gauches.

Certaines eombinaisons hybrides donnent lieu à des phénomènes analogues. J'en donne un exemple foumi par des Tabaes.

Vicotiana rustica L. et $N$. paniculata L. sont des sortes assez différentes par le port, la végétation, la ramification des axes et la forme des fleurs pour que Linné les ait distinguées comme de bonnes espèces. En réalité, elles rentrent dans la spéciéité au troisième degré de NAUdin, en ce sens que les hybrides sont stériles, mais peuvent facilement être féeondés par le pollen de l'un ou de l'autre parent. Lorsqu'on féconde les ovaires de l'hybride (Nicotiana paniculata $\times$ N.rustica) par le pollen de $N$. rustica, la produetion de bonnes eapsules est constante, mais ehaeune d'elles renferme au plus 40 graines bien formées, alors que les capsules des parents en renferment au moins 400. Continuons l'expérience et féeondons les ovaires de l'hybride second par le pollen de rustica, la féeondité augmente et l'on obtient une moyenne de graines par fruits dépassant la eentaine ; avec une ou deux opérations de plus, on parvient à la fertilité complète et la deseendanee est du type pur rus. tica. Ainsi, l'influence maternelle de l'hybride premier est abolie après 4-5 épurations qui eonsistent évidemment en ce que les germes des pollens rustica, en présence des solutions collö̈des des saes embryonnaires du paniculata, puis du (paniculata $\times$ rustica), puis du (paniculata $\times$ rustica) $X$ rustica, ete., déterminent un virage en quelque sorte régulier et progressif dans le sens du germe régulièrement 
introduit. L'opération inverse permettrait de substituer à l'espèce rustica l'espèce paniculata. L'épreuve suggérée par l'expériene de Gernez fournit done un moyen, précieux pour les biologistes, pour reconnaître expérimentalement la nature profonde, ou la nature superficielle des spéciéités.

Les croisements de variétés régressives, telles que le Maïs à grains amylacés avec le Maïs à grains sucrés, donnent lieu à des phénomènes parallèles, selon le choix de l'une ou l'autre variété comme plante maternelle. Ainsi Zea Mays amylacea femelle fécondée par $Z$. M. saccharata donne uniquement, en première génération, des descendants du type amylacea ; pollinisant constamment cet hybride et ses descendants avec du pollen amylacea pur, il n'apparaîtra jamais de grains saccharata. Mais le même hybride initial pollinisé par le pollen saccharata donnera à la deuxième génération une moitié des grains amylacea, une moitié saccharata, ces derniers épurés, les premiers possédant les caractères et les propriétés de l'hybride premier. On ne pourra éteindre cette propriété complètement dans la suite des générations ; il n'y a pas métamorphose d'un type en l'autre, mais substitution de qualités indifférentes à l'espèce.

Dans le cas des Nicotiana, la suite des épurations est accompagnée d'un déchet surtout sensible avec l'hybride de première génération, puisque le nombre des graines formées est réduit an dixième; ce déchet est d'ailleurs en corrélation avec la vigueur exceptionnelle de l'hybride premier, dont les premières fleurs formées tombent à l'état de boutons sans s'ouvrir ; ce n'est qu'à la limite, à la troisième ou à la quatrième introduction de germes purs, que la fécondité redevient normale, en même temps que les individus rentrent dans l'espèce qui a fourni le pollen pour les féconda- 
tions successives; on peut d'ailleurs dire, ce qui est exact, que le déchet mesure la part des éléments de l'autre espèce, qui diminue progressivement.

Dans le cas du Maïs, pas de stérilité même partielle, pas de déviations notables, ni dans le port, ni dans la taille, ni dans la succession des fleurs; parents et descendants diffèrent seulement par le caractère de la condensation des réserves de l'albumen sous la forme d'amidon on sous la forme de dextrine. Et e'est dans cette seule divergence qu'il faut chereher l'analogie avec les phémomènes de cristallisation des solutions sursaturées ; l'élément sucré renfermé par le grain de pollen donne à la masse qui constituera l'albumen le mode de condensation qui lui est propre ; il agit exactement de la même façon qu'un germe cristallin द̇e formiate de strontiane. J'en conclus que la nature des réserves est indépendante de l'équilibre molécnlaire spécifique.

J'ai donné cette suite d'exemples pour montrer combien est grande la différence de comportement des divers hybrides, combien les analogies que j'ai établies entre les plénomènes de la eristallisation et les phénomènes de l'hérédité peuvent être utiles pour orienter l'analyse des divers cas particuliers.

Lorsque PASTEur combine deux corps actifs, l'acide tartrique droit et la cinchonine, il fait un véritable hybride d'espèces ; il détruit ou ébranle la dissymétrie moléculaire propre à l'acide tartrique droit et il en retire par un procédé simple l'autre sous-espèce, celle qui dévie à gauche. Il a fallu l'intime combinaison de deux corps moléculairement différents, l'acide tartrique et la cinchonine, pour provoquer l'état de dissociation: le groupement moléculaire nouveau 
n'est pas quelconque ; il est l'image dans une glace du premier. Il y a eu transformation d'espèce dans les conditions d'équilibre propres à l'espèce acide tartrique.

Lorsque GERnez transforme, par la répétition de l'ensemencement de germes cristallins droits, le formiate de stron. tiane gauche en un formiate de strontiane droit, il n'a pas modifié l'équilibre moléculaire de corps. Pastreur a, en effet, montré que l'hémiédrie dans ce cas n'est pas dans la molécule ; elle dérive de l'agencement des molécules dans le cristal. En conséquence, le germe spécifique, qu'il soit droit ou gauche, n'a ici d'autre effet que de construire un cristal à son image sans altérer en quoi que ce soit la moléeule ; d'ailleurs l'opération n'a pas de déchet.

Lorsque je transforme, après KøLREUTER, après NAUDIN, par des pollinisations répétées avec Nicotiana rustica, la plante paniculata en plante rustica, je n'ai pas altéré la molécule, l'essence spécifique du Vicotiana; j'ai fait virer une masse malléable dans son organisation, mais intacte quant à ses qualités spécifiques, en une forme différente ii est vrai, mais qui n'en est pas moins une variété de Nicotiana paniculata. Autrement dit, le seul fait que par la pollinisation seule et sans altération notable de la fécondité on peut passer d'une espèce à l'autre, par des épurations successives, démontre que les deux espèces affines ne sont pas fondamentalement distinctes, qu'elles sont d'un degré de spéciéité plus élevé que les formes qui, par leurs croise. ments, domnent des hybrides presque toujours stériles. Par ce rapprochement, j'approfondis l'étude des spécéités à divers degrés.

J'ai appliqué cette méthode pour distinguer dans les Blés, dans les Orges, dans les Lins, les spéciéïtés différentes, et j'ai obtenu presque à coup sûr, dès la première génération 
hybride, la distinction entre les espèces vraies et les formes ou variétés d'une même espèce.

Voici d'ailleurs une autre preuve. Dans les eristaux dimorphes, la molécule n'est pas profondément modifiée ; il n'y a pas de déchet quand, par son procédé, Gernez montre qu'un changement de germes suffit pour passer d'une forme à l'autre (1). Dans un tube en $U$, il verse du soufre en dissolution dans le sulfure de carbone, qui s'évapore, et il protège les ouvertures du tube pour éviter la chute accidentelle de poussières de soufre solide; le contenu du tube reste en état de surfusion. Ceci fait, il laisse tomber un eristal de soufre octaédrique dans une des branches, un cristal de soufre prismatique dans l'autre; il y a cristallisation presque immédiate dans les deux branches, plus lente seulement dans la branche octaédrique ; la masse entière se partage en cristaux des deux systèmes qui viennent en contact.

Hugo dF VRIES (1900) part d'une lignée stable de Maïs à grains sucrés ; il isole un épi femelle ; puis, lorsque les stigmates sont bien développés, il les saupoudre avec un mélange à parties égales de pollen de Maïs sucré et de pollen de Maïs amylacé. $\mathrm{A}$ la récolte, il trouve sur l'épi autant de grains de Maïs sucré que de Maïs amylacé ; le hasard de la répartition des polleus donne côte à côte les deux états sans grains avortés.

Il y a plus : jusqu'ici nous avons fait intervenir des germes pour obtenir à volonté l'une ou l'autre variété de

1. - Sur les eirconstances de la production des deux variétés prismatique et octaédrique du Soufre, C. R. Acad. Sc., 1876, t. LXXXVIII, p. 217. 
l'espèce. S'il s'agit d'adaptation double, l'intervention de facteurs physiques seuls suffira; des changements dans le milieu ou le frottement, le froid, aboutissent à la transmutation provoguée d'ordinaire par les germes.

Gernez l'établit par le soufre. Il semble qu'il ait été conduit à cette série d'expériences par l'observation, familière aux cristallographes, de l'accélération de la cristallisation d'une solution sursaturée lorsqu'un point de la masse est en contact avec mn corps froid, et il reproduit cette expérience pour le soufre lni-même. Plaçons un morceau de glace au contact du verre du tube en $U$ renfermant le sonfre en surfusion dont il est question plus haut, il y a prise rapide de la partie séparée diu morceau de glace par la paroi de verre ; puis tout le soufre se transforme en une masse cristalline composée uniquement de prismes, état instable, qui se transformera lentement plus tard' en octaèdres. On suit, à partir de l'état de surfusion, cette fois, les modifications étudiées dans le deuxième chapitre sous le titre des adaptations doubles.

Mais, ajoute GERNEZ, une action purement mécanique peut donner le même résultat. Une baguette effilée de verre inactive est plongée dans la solution sursaturée de soufre; si l'opération est faite avee lenteur, sans mouvements brusques, l'état liquide persiste; si l'on frotte un peu vivement la pointe de la baguette contre la paroi du verre, il y a prise au point du choc et les cristaux obtenus sont du type prismatique; le frottement violent détermine ici les effets du contact d'un corps froid ou de l'addition du germe instable.

Une nouvelle série d'observations (1) est communiquée

1. - D. Gernez : Influence qu'exerce une action mécanique sur la production des divers hydrates dans les solutions sursaturẻes, C. R. Ac. Sc., 1877, t. LXXXlV, p. 1389. 
l'année suivante à l'Académie. Gernez constate que c'est bien l'état de dimorphisme qui domine les manifestations particulières de la sursaturation et de la cristallisation par action mécanique. Il fait remarquer, dans un préambule, que les solutions concentrées de certains sels cristallisent spon. tanément; pour une certaine limite de concentration à la température ordinaire, on n'obtient que des cristaux du sel le moins hydraté ; par exemple, s'il s'agit de sulfate de soude qui donne deux hydrates $\mathrm{SO}^{4} \mathrm{Na}^{2}+7 \mathrm{H}^{2} \mathrm{O}$ et $\mathrm{SO}^{4} \mathrm{Na}^{2}+10 \mathrm{H}^{2} \mathrm{O}$, le dépôt sera uniquement formé de $\mathrm{SO}^{4} \mathrm{Na}^{2}+7 \mathrm{H}^{2} \mathrm{O}$. Naturellement, l'ensemencement d'un germe de $\mathrm{SO}^{4} \mathrm{Na}^{2}+10 \mathrm{H}^{2} \mathrm{O}$ ferait cesser, lui aussi, l'état de. sursaturation en stabilisant la forme en $\mathrm{SO}^{4} \mathrm{Na}^{2}+10 \mathrm{H}^{2} \mathrm{O}$.

Prenant une solution concentrée, en état de sursaturation qui tend à donner le sel le moins hydraté, GERNEz introduit une tige rigide inactive dans la masse et la frotte vivement contre la paroi du vase; il pourra se faire qu'on détermine la production de cristaux. Cette action ne réussit pas avec une sursuration peu avancée; elle est d'autant plus facile que la concentration du liquide est plus voisine de l'état de cristallisation spontané. La solution de sulfate de soude sursaturée et maintenue au-dessous de 8 degrés donne la. cristallisation $\mathrm{SO}^{4} \mathrm{Na}^{2}+7 \mathrm{H}^{2} \mathrm{O}$ avec un frottement ordinaire.

Mais il y a d'autres cas possibles. La solution d'acétate de soude, qui fournit deux hydrates, avec 3 ou avec 6 molécules d'ean, donne, dans des conditions analogues et après frottement, l'hydrate à 6 molécules d'eau.

Enfin, le chlorure de calcium donne simultanément les deux hydrates si l'on a soin d'opérer comme il suit : amener lentement la solution à l'ébullition pour se rapprocher de l'état de saturation, éviter la formation superficielle de cris- 
taux à 6 molécules d'eau qui ont une tendance à s'y former et ramener la solution à $15^{\circ}$ en notant qu'elle reste bien limpide. Si l'on frotte très légèrement avec une tige d'acier inactive, on voit se former à la surface du liquide des aiguilles feutrées de chlorure à 4 molécules d'eau ; il faut attendre que la chaleur dégagée par cette formation se dissipe et, toujours à 15 degrés, on plonge la baguette au fond $d u$ tube ; en frottant vigoureusement la paroi, on obtient des cristaux à 6 molécules d'eau en profondeur. Une action trop vive ferait apparaître une masse de cristanx à 6 molécules d'eau dans toute la masse, masquant la formation peu stable et locale de cristaux à 4 molécules.

Il en résulte une démonstration évidente de deux modes d'activité bien distincts des germes déposés dans les solutions sursaturées. Lorsque la substance en saturation n'a pas de dissymétrie moléculaire, mais présente, à cause de sa position limite par rapport à deux systèmes d'équilibres cristallins, un dimorphisme, l'addition de cristaux germes de l'un ou l'autre système font évoluer la masse entière en l'un ou l'autre type de cristal. Les résultats obtenus ne sont pas dus à la qualité spécifique des germes, mais à leur état; d'autres corps présentant les deux états possibles agiraient à la façon de germes propres ; des ébranlements mécaniques, des actions purement physiques peuvent être substitués aux germes. Les états des substances dimorphes sont en quelque mesure indépendants de la constitution moléculaire de l'espèce.

Mais lorsque les états d'une même substance chimique sont liés à la constitution de la molécule, ces états sont, au même titre que des corps simples introduits dans le composé, des éléments propres à l'espèce; ils sont même plus 
intimement liés à l'espèce que ne le sont les substances capables de se substituer l'une à l'autre en maintenant l'isomorphisme, e'est-à-dire en laissant intacte la configuration géométrique de la molécule.

Les germes qui apportent avec eux une dissymétrie fon. damentale provoquent la condensation de toute la matière dissoute qui possède le même arrangement moléculaire ; ils laissent intact le reste. Le résidu ne peut être transformé dans la dissymétrie opposée que si on le combine à des corps doués eux-mêmes d'une dissymétrie propre et d'affinités spéciales; il faut nécessairement passer par la phase de désagrégation moléculaire, qui entraînera le plus souvent l'impossibilité de cristalliser. Par hasard, et les agents physiques aidant, il pourra se faire que l'équilibre inverse naisse : e'est une mutation. D'ordinaire, la mutation cherchée n'apparaîtra pas seule; il se formera d'autres Équilibres qui ne seront pas quelconques. Il y aura pulvérisation en sous-espèces dans l'espèce même et, en même temps, substitution, ou addition, on neutralisation de quelques propriétés des germes; ce n'est pas du transformisme au sens habituel du mot, c'est du polymorphisme spécifique.

A un équilibre stable, quoique dissymétrique, correspond nécessairement un autre équilibre stable, image du premier dans un miroir. Les êtres vivants, dans la conception de PASTeur, sont des complexes de plusieurs substances dissymétriques ; ils ne sont jamais stables, ce qui impliquerait la mort. Mais la permanence de certains équilibres à travers les générations sexuées entraîne la possibilité de la permanence des équilibres inverses. La possession de deux formes d'équilibre d'une même espèce facilite l'acquisition des autres équilibres possibles ; e'est mettre en évidence à la fois l'intérêt des mutations et des hybridations. 
CHAPITRE V

\section{LE MILIEU ET LA MIGRATION DES GERMES}

Les relations du milien avec les êtres vivants qui le peuplent ont frappé de tout temps l'imagination des voyageurs, des géographes, des naturalistes. Aussi ne peut-on attribuer à DARwin l'origine même de l'idée que les circonstances ambiantes, que les conditions générales d'élevage modèlent en quelque manière les êtres qui les subissent. Mais on peut affirmer qu'avant lui les documents épars n'avaient pas le caractère de généralité, ni la force probante, que tout le monde accorde, après 1860 , à la corrélation étroite qui unit les formes et les habitats.

Une circonstance exceptionnelle a mis Darwin sur la voie qui devait fournir les traits essentiels de la doctrine du transformisme. Au cours d'un voyage autour du monde sur Le Beagle, dont la première relation fut publiée en 1839, DARwIN explore l'archipel des Galapagos, sibué sous l'Equateur, à 500 ou 600 milles à l'ouest des côtes de l'Amérique ; il reconnaît leur nature volcanique récente et, d'après la composition et la répartition des tufs qui en couvrent les bords, il démontre que les dix îles principales de l'archipel doivent leur origine à des cratères formés sous l'eau, émergés et recouverts de boues précédemment accumulées dans le fond de la mer. Il arrive à se représenter les débuts de la vie sur ces terres nouvelles et, par une étude attentive des coquillages, des animaux aériens et des plantes, il acquiert, presque 
malgré lui, la conviction qu'il s'est formé sur place un monde nouveau à l'image du sol qui vient de surgir de l'Océan. Bien que l'archipel soit situé sous l'Equateur, le climat n'y est pas extrêmement chaud; les eaux voisines sont dérivées du grand courant polaire du Sud et y maintiennent une couverture de nuages fort bas ; il y pleut rarement ; aussi les zones basses des îles sont-elles stériles ; la végétation n'est prospère qu'à mille pieds et au-dessus.

Le docteur Hooker, qui étudia les récoltes de DARwin, signale, dans l'ensemble des îles Galapagos, 185 espèces portant des fleurs et 40 cryptogames; sur les 185 espèces, 100 sont des espèces non décrites, dont l'existence paraît limitée à l'archipel ; 10 au moins ont été importées récemment d'Amérique et il est probable que le reste, soit 75 espèces, en proviennent aussi. Mais la majorité des êtres sont des productions indigènes récentes. " Rien de frappant, dit DARwrs, comine de se voir entouré par de nouveaux oiseaux, de nouveaux reptiles, de nouveaux coquillages, de nouveaux insectes, de nouvelles plantes, et cependant de se sentir transporté, pour ainsi dire, dans les plaines tempérées de la Patagonie, ou dans les déserts si chauds du Chili septentrional, par d'innombrables détails insignifiants de conformation et même par le ton de la voix et le plumage des oiseaux. Comment se fait-il que, sur ces petits îlots, qui tout dernièrement encore, géologiquement parlant, devaient être recouverts par les eaux de l'Océan, îlots formés de laves basaltiques, et qui diffèrent par conséquent du caractère géologique du continent américain, outre qu'ils sont situés sous un climat particulier, comment se fait-il, dis-je, que sur ces petits îlots les habitants indigènes, différant et par le nombre et par l'espèce de ceux du continent et réagissant par conséquent l'un sur l'autre de façon différente, aient 
été créés sur le type américain ? Il est probable que les îles $\mathrm{du}$ Cap-Vert ressemblent par toutes leurs conditions physiques aux îles Galapagos beaucoup plus que ces dernières ne ressemblent physiquement à la côte de l'Amérique ; cepen. dant les habitants indigènes des deux groupes sont absolument dissemblables; ceux des îles du Cap-Vert portent la marque de l'Afrique, de même que ceux des îles Galapagos portent celle de l'Amérique " (1).

L'examen des espèces dans le détail fournit des résultats encore plus frappants. Le gouverneur de l'archipel, Lawson, attire l'attention de DARwin sur le fait que les indigènes distinguent les unes des autres les Tortues qui vivent sur les bords de l'une ou de l'autre des îles, distantes de 50 à 60 milles. Faisant la statistique des Légumineuses, Darwin trouve que sur les 38 espèces qui ont été récoltées dans l'île James et ne se trouvent en aucune autre partie du monde que dans l'archipel Galapagos, 30 sont strictement cantonnées dans cette île ; sur les 26 espèces de l'île Albemarle particulières aux îles Galapagos, 22 ne se trouvent que dans cette île. Les espèces de Composées sont tout particulièrement localisées.

Les lecteurs de l'Origine des espèces (1859), de la T'ariation des animaux et des plantes (1868), ouvrages qui renferment à la fois les conceptions les plus hardies, les preuves les plus variées de la dérivation des espèces les unes des autres, et la démonstration de la lutte pour l'existence d'êtres analogues croissant dans un même milieu limité, ne manqueront pas de trouver dans les observations faites en 1835 par DaRwix l'expression de deux phénomènes qui

1. - Ch. Darwin : Voyage d'un naturaliste autour du monde fait à bord du navire Le Beagle, de 1831 à 1836. Trad. Barbier, p. 421. 
s 'imposent naturellement à l'esprit. La flore et la faune des îles Galapagos portent le eachet propre aux productions vivantes de l'Amérique parce qu'elles en dérivent directement; les transports par le vent, par l'eau, peut-être par' l'homme, suppléent à l'absence des germes d'espèces aériennes sur les îles volcaniques naissant au sein de l'Océan. "Selon CoLnetr, dit DARwin, des bambous, des cannes à sucre, des noix de palmier, en un mot des bois de toute espèce, sont souvent amenés par les courants sur les côtes sud-est de ces îles. ") D'autre part, les caractères particuliers à la grande majorité des espèces et surtout la ségrégation des espèces elles-mêmes sur des îles différentes, quoique relativement rapprochées, puisqu'elles sont en vue les unes des autres, indiquent que les échanges de germes, quoique fréquents, n'entraînent pas nécessairement l'uniformité des espèces. Les espèces installées dans un domaine le conservent malgré les intrusions de rares individus qui $y$ auraient donné une large postérité s'ils avaient trouvé le terrain libre ; les adaptations répétées et la multiplication intensive des premiers habitants suffisent pour limiter ou retarder la coneurrence qui se fera néanmoins avec âpreté tôt ou tard. D'où l'idée que Darwin visita les îles Galapagos dans une période de propagation et d'extension des espèces et non dans la phase de concurrence vitale caractéristique des flores et des faunes des anciens continents.

Bien qu'il soit évident que les îles Galapagos aient une origine très récente par rapport à la formation des grands continents, il est difficile de leur assigner un âge et de dire si cinq cents siècles, des milliers sans doute, ne se sont pas écoulés depuis que ces îlots volcaniques ont émergé de l'Océan. Quelque merveilleux que soient les progrès réalisés 
par l'homme dans l'analyse et la régularisation des forces naturelles, il est impossible d imaginer la réalisation, sur une échelle même réduite, d'effets semblables à ceux que l'imagination soupçonne exister à l'origine de la répartition et de l'évolution des gerınes, introduits par le hasard du continent américain sur ces terres vierges. Je le répète, les preuves ảu transformisme sunt toutes dans la répartition dans le temps et dans l'espace d'êtres ofirant des analogies suffisantes pour qu'on soit autorisé à leur attribuer des origines communes. Les relations historiques et géographiques des objets n'auront jamais, au point de vue de la certitude, la valeur de quelques expériences bien faites et, pour cette raison, les modèles qui doivent inspirer les biologistes pour l'édification solide d'une seience des origines ne peuvent ètre une simple imitation dess phénomènes naturels. La durée de la vie humaine, celle même de l'espèce humaine, ne compte pas dans l'étendue des temps géologiques, et il faut renoncer à réaliser une image, même imparfaite et limitée à un petit groupe d'êtres, des étapes qui ont marqué la succession des elasses, des ordres, des familles, des genres et des espèces, répartis à la surface du globe.

Pasteur, aidé du microscope qui permet de distinguer les formes d'organismes dont la taille ne dépasse pas quelques millièmes de millimètres, a le premier défini des mondes vierges de tout germe, et mis à notre disposition, en quantité illimitée, des milieux qui offrent du point de vue logique des sécurités supérieures à celles que présentaient les îles Galapagos surgissant du fond de l'Oeéan ; il a réussi à isoler des germes dans des gouttes de liquides nutritifs et a pu les transporter séparément daus ces milieux à l'exclusion de tous les autres germes vivants. Ce faisant, il a démontré leur spécificité, prouvant qu'il existe, entre les différents germes 
isolés, des identités qui les font réunir dans un même groupe, ou des différences stables et constantes qui en font des prototypes d'espèces distinctes; il a étudié la lutte pour la place, pour la nourriture, de ces différents groupes et même leur propre autophagie, lorsque les circonstances étaient très défavorables à la croissance; il a mis en évidence leurs adaptations, sans réussir pourtant à établir leur transmutation. Je vais passer rapidement en revue les principaux traits de ces remarquables recherches, en suivant autant que possible l'ordre chromologique qui montre leur enchaînement.

\section{$* *$}

En présentant à l'Académie des Sciences, le 30 novembre 1857, son premier Mémoire sur les fermentations, Louis Pasteur s'excuse presque de traiter un sujet de chimie biologique bien éloigné en apparence de ses premiers travaux. Il nous découvre ainsi l'essentiel de l'enchaînement des idées qui vont donner une direction nouvelle à toutes les recherches faites depuis sur les altérations des matières organiques.

PASTEur venait (1855) d'établir que l'alcool amylique, résidu de la fermentation des jus de betteraves, était une matière complexe formée de deux alcools distincts, l'un déviant à gauche le plan de polarisation de la lumière, l'autre inactif; préparant le sulfamylate de baryte avec ces deux corps, il trouve une exception à la loi de corrélation de l'hémiédrie et du phénomène rotatoire; il ne pouvait l'accepter sans résistance et en fait l'étude approfondie.

Poursuivant la recherche de la cause initiale du pouvoir rotatoire, il se demande s'il n'a pas celui du sucre ponr 
origine; il rejette cette explication parce que le " groupe moléculaire de l'alcool amylique est trop distant de celui du sucre pour que, s'il en dérive, il en retienne une dissymétrie d'arrangement de ses atomes " (1). D'autre part, " on voit toujours ces alcools prendre naissance dans l'opération de la fermentation ", laquelle est ramenée par PASTEUR à un phénomène accessoire de la vie ; or, les êtres vivants créent des dissymétries moléculaires. Il importait done d'établir que les fermentations ne se produisent que par l'intervention d'êtres vivants.

Prenant comme exemple l'acide lactique du petit lait aigri, il montre la liaison des produits dérivés et des ferments spécifiques : " de même qu'il existe un ferment alcoolique, la levure de bière, que l'on trouve partout où il y a du sucre qui se dédonble en alcool et en acide carbonique, de même il y a un ferment particulier, une levure lactique, toujours présente quand du sucre devient acide lactique ". (Fig. 17, la.)

D'après LieBig, dont l'influence était prépondérante à l'époque, les fermentations consistaient en des désagrégations de molécules grosses, se disloquant en molécules plus petites, mais encore complexes. La fermentation lactique était un phénomène type de cette désagrégation; de plus, elle fournissait comme produit accessoire abondant le mystérieux alcool amylique, pierre de touche de la valeur de ses conceptions. Pasteur l'étudia en chimiste, pesant les corps en présence, analysant les gaz émis, cherchant les conditions les plus favorables à la cristallisation du lactate de chaux, et surtont en biologiste, qu'il était devenu sans le savoir, décou-

1. - Mémoire sur la fermentation appelée lactique, Ann. de Ch. et Ph., 3e série, t. LII, p. 405. 
vrant la cellule vivante active et les milieux favorables à sa multiplication.

Dans les fermentations lactiques ordinaires, on observe une matière grise formant quelquefois zone à la surface du đépôt, ou collée aux parois supérieures des vases. Mélangée en très faible proportion au caséum et à l'écume crayeuse, on pourrait souvent en méconnaître l'existence. “C'est elle, néanmoins, qui joue le principal rôle. Je vais, dit Pasteur, tout d'abord indiquer le moyen de l'isoler, de la préparer à l'état de pureté. )
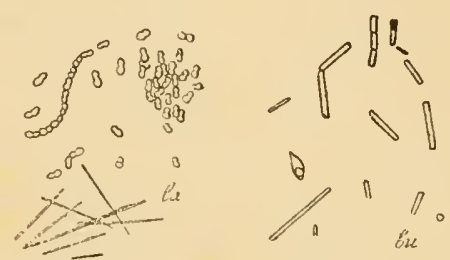

Fig. 17. - Ferment lactıque, a $a$, avec cristaux en aiguilles de lactate de chaux ; ferment butyrique montrant la formation de spores, bu ; très grossis (d'après Deccavx).

Un extrait, soluble après ébullition, de levure de bière dans quinze à vingt fois son poids d'eau, est filtré avec soin ; on y fait dissoudre 50 à 100 grammes de sucre par litre, on chasse l'air du flacon par un courant d'acide carbonique, on ajoute de la craie et on porte à l'étuve à $30^{\circ}$ ou $35^{\circ}$. Une trace de la matière grise, extraite d'une bonne fermentation lactique ordinaire, y est semée. Le lendemain, la fermentation lactique est parfaitement caractérisée ; lorsque la craie a disparu, PASTEUR obtient par évaporation une cristallisation abondante de lactate de chaux et l'ean-mère contient des quantités variables de butyrate de la même base.

Contrairement à ec qui a lieu d'ordinaire, la fermentation 
est très rapide et régulière, lorsque le ferment lactique se développe seul. Le plus souvent, les milieux renferment énormément de matières inutiles, gluten, caséum, fibrine, membranes, tissus..., " qui ne deviennent un aliment pour le ferment lactique qu'après s'être putréfiées, altérées, au contact de végétations ou d'animalcules qui ont rendu leurs éléments solubles et assimilables...

"Si l'on ne sème aucun ferment dans un mélange d'eau sucrée, de matière albumino:̈de et de craie, on a généralement plusieurs fermentations parallèles avec leurs ferments respectifs et des animalcules qui paraissent dévorer les petits globules de ces ferments. L'addition préalable d'un ferment déterminé et pur favorise beaucoup la production d'une fermentation unique et correspondante, sans l'assurer dans tous les cas. On peut comparer ce qui se passe dans les fermentations à ce que nous offre un terrain dans lequel on ne place aucune semence. On le voit bientôt chargé de plantes et d'insectes divers qui se nuisent mutuellement.

"La pureté d'un ferment, son homogénéité, son dévelop. pement libre, sans aucune gêne, à l'aide d'une nourriture très bien appropriée à sa nature individuelle, voilà l'une des conditions essentielles d'une bonne fermentation. "

La levure de bière (fig. 18, le), les infusoires, sont les compétiteurs naturels du ferment lactique. La première agit fort mal en milieu alcalin ; si on n'ajoute pas de craie, on peut être assuré que le lendemain la fermentation sera alcoolique; si on rend le milieu neutre ou un peu alcalin: ferment lactique et levure agiront simultanément, et le liquide, examiné au microscope, montrera, mêlés anx globules de levure, une quantité considérable de petits globules de ferment lactique. Le jus d'oignon semble inoffensif pour ce dernier ; il entrave le développement de la levure de bière 
et paraît nuire également aux infusoires ; c'est le procédé par lequel Pasteur espère (1857) déterminer constamment et facilement la fermentation lactique sans complication de ferments ou d'infusoires étrangers aux phénomènes essentiels.

La même année, Pasteur décrit (1) un autre procédé qui est actuellement encore universellement adopté lorsqu'on veut isoler un Champignon ou une Bactérie dans un milieu vivant.

Prenant deux parties égales de levure fraîche larée à
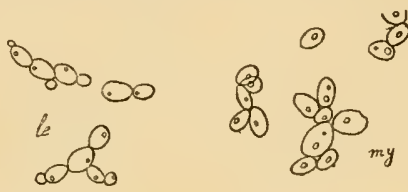

Fig. 18. - Levure de bière jeune, le; fleur du vin on Mycoderma, my (d'après Decuaux).

grande eau, l'une est mise en fermentation avec de l'eau sucrée pure, l'autre est bouillie avec beaucoup d'eau; on filtre eette décoction pour enlever les globules tués; on y ajoute la même quantité de sucre que dans le premier lot et une trace de levure fraîche. Les globules semés bourgeonnent et la désagrégation du sucre est sensible au bout de quelques heures. "Voici le fait qu'il importe de noter. En déterminant par cet artifice l'organisation en globules de la partie soluble de la deuxième portion de levure, on dédouble un poids de sucre considérable. Je rapporterai les résultats d'une expérience : 5 grammes de levure ont fait fermenter

1. - Mémoire sur la fermentation alcoolique : C. R. Acad. Sciences, 21 décembre 1857, p. 1032. 
en six jours 12,9 grammes de sucre et étaient épuisés. La partie soluble d'une égale portion de 5 grammes de la même levure a fait fermenter 100 grammes de sucre en neuf jours et la levure développée par la semence était également épuisée. ")

Autrement dit, ce n'est pas la quantité de semence de levure qui domine le phénomène de la fermentation, c'est la quantité d'éléments solubles dans le milieu qui peut être organisé par des traces de levure; la fermentation apparaît alors comme un accessoire de l'édification, à partir de quelques germes, d'une masse considérable de dérivés de ces germes. Et, plus loin, Pasteur insiste : “ Les théories de la fermentation partent de ce principe que le ferment ne cède rien et ne prend rien à la matière fermentescible. Je vais démontrer au contraire que la levure emprunte quelque chose au sucre, que le sucre est un de ses aliments, qu'il n'y a pas équation entre les quantités d'alcool, d'acide carbonique (d'acide lactique) et le poids total du sucre devenu incristallisable... la différence de poids est variable... et les pertes importantes sont placées du côté de la portion de levure qui pèse le plus...

" Le dédoublement du sucre en alcool et en acide carbonique est un acte corrélatif d'un phénomène vital, d'une organisation de globules, organisation à laquelle le sucre prend part directe, en fournissant une portion des éléments de la substance des globules. »)

Ces conclusions sont actuellement évidentes pour tout le monde ; elles furent combattues pendant dix années par les chimistes les plus éminents de l'époque. Pasteur y a été conduit sans nul doute par ses études de cristallographie; l'introduction du ferment dans une eau-mère renfermant quantité de substances de la nature même dẻ celles qui exis- 
tent dans le ferment rappelle à s'y méprendre les conditions les plus favorables pour obtenir les cristallisations les plus abondantes et les plus régulières (1).

Une année plus tard, après avoir fait connaître la production constante, au cours de la fermentation alcoolique, de quantités pondérables d'acide succinique, de glycérine, PASTEur signale à Dumas, qui l'annonce à l'Académie, que la levure peut détruire par la fermentation des milieux composés de matériaux eristallisés ou calcinés : "Dans une solution de sucre pur, je place d'une part un sel d'ammoniaque, par exemple du tartrate d'ammoniaque, d'autre part la matière minérale qui entre dans la composition de la levure de bière, puis une quantité pour ainsi dire impondérable de globules de levure frais. Chose remarquable, les globules semés dans ces conditions se développent, se multiplient et le sucre fermente, tandis que la matière minérale se dissout peu à peu et que l'ammoniaque disparaît. En d'autres termes, l'ammoniaque se transforme en la matière albuminoïde complexe qui entre dans la constitution de la levure, tandis que les phosphates donnent aux globules nouveaux leurs principes minéraux. Quant au carbone, il est évidemment fourni par le sucre.

"Vient-on à supprimer dans la composition du milieu, soit la matière minérale, soit le sel d'ammoniaque, soit ces

1. - Disons en passant que la culture des microorganismes parasites des animaux et des végétaux est facilitée par la préparation de bouillons composés pour partie des éléments constitutifs de l'hôte. Nous verrons plus loin que le bouillon de muscles de poule favorise plus qu'aucun autre milieu la croissance du bacille du Choléra des poules ; cette technique est inspirée évidemment de la même conception originale. 
deux principes à la fois, les globules semés ne se mutiplient plus du tout et il ne se manifeste aucun mouvement de fermentation 》(1).

Moins de deux mois s'écoulent et, cette fois, c'est le milieu favorable à la fermentation lactique qui est fabriqué de toutes pièces avec du sucre candi pur cristallisé, une petite quantité d'un sel ammoniacal, des phosphates et du carbonate de chaux précipités; le plus souvent la levure lactique se développe, donnant une dépôt de lactate de chaux et associée d'ordinaire à des infusoires. "Si I'on supprime tout contact avec l'air commun, ou si l'on porte à l'ébullition le mélange de sucre, de sel d'ammoniaque, de phosphates et de craie, pour n'y laisser rentrer que de l'air chauffé au rouge, il ne se forme ni levure lactique, ni infusoires, ni fermentation quelconque... Sur ce point, la question de la génération spontanée a fait un progrès. ")

Avant de l'examinel, faisons remarquer que Pasteur étudia, avec les mêmes méthodes et le même succès, la fermentation de l'acide tartrique (1858), la fermentation butyrique (1861), dont l'agent est un bacille anaérobie (fig. 17, bu), la fermentation acétique (1861), les conditions nécessaires et suffisantes pour assurer la fabrication normale, sans maladies ni irrégularités, du Vin (1866), de la Bière (1873). Partout, on reconnaît la même méthode expérimentale, le même souci des détails, la conviction qu'il faut donner au germe pur convenablement choisi le terrain, c'est-à-dire le milieu de culture, permettant son épanouissement complet en quelques jours. La découverte de l'atténua-

1. - Nouveaux faits concernant l'histoire de la fermentation alcoolique : C. R. Acad. Sciences, 20 décembre 1858, p. 1011. 
tion des virus dérive, comme je le montre plus loin, de la sûreté de la technique des cultures de microorganismes dont je viens d'exposer les traits fondamentaux.

Les découvertes de PASTEUR, relatives au milieu le plus favorable au développement des ferments, eurent des conséquences énormes sur l'étude de la nutrition des végétaux supérieurs. La Chimie végétale paraît débuter avec la constatation du célèbre physicien MARIOTTE (1679), qui écrit dans une lettre sur le sujet des plantes : "Prenez un pot qui contienne de sept à huit livres de terre ; semez-y n'importe quelle plante; elle trouvera dans cette terre et dans l'eau de pluie qui y pénètre tous les principes dont elle se compose lorsqu'elle a atteint son complet développement. On pent semer dans cette terre trois ou quatre mille espèces végétales ; si les sels, les huiles, les matières terreuses dont se compose chaque espèce végétale étaient de nature différente, tous ces principes devraient se trouver réunis dans la petite quantité de terre dont nous avons parlé plus haut et dans l'eau de pluie qui arrose cette terre durant trois ou quatre mois, ce qui est impossible. ") Plus tard, de Saussure (1804) insiste tout particulièrement sur l'importance des cendres des corps végétaux comme éléments de la bonne nutrition; jusqu'à lui, on les supposait introduites accidentellement dans les organes à la façon de la silice qui incruste les chaumes des Graminées et donne la rigidité et la dureté aux tiges des bambous. La théorie des engrais, perfectionnée par Boussingault, Georges Ville, Isidore Pierre, prenait à l'époque où PASTEUR étudiait les aliments de choix des ferments, un développement magnifique qui permit de décupler les rendements.

Sous sa direction, RAULin (1870) poursuit pendant dix 
années ses Etudes chimiques sur la végétation, où l'on retrouve, exposée par un esprit indépendant et très perspicace, la démonstration précise de l'importance des impuretés, de ces traces de corps dont la présence ou l'absence modifie totalement les conditions de la croissance et la masse des matériaux construits à partir du même poids de germes. MiM. G. Bertrand, Javillier, Mazé, et, plus récemment, Mis. Maquenne et Demoussy, ont porté à un haut degré de perfection la technique des cultures aseptiques des végétaux supérieurs, et montré l'intervention décisive d'éléments dont il est impossible d'évaluer le poids. Si PASTEur avait su que les traces de chaux laissées dans l'eau par le contact du verre $d u$ ballon suffisaient pour altérer le développement de la racine du Pois, il aurait trouvé incontestablement dans ce fait l'expression la plus justifiée de ses déductions relatives à l'affinité des germes pour des milieux strictement appropriés à leur nature et à l'instabilité des conditions naturelles de la vie.

La voie est largement ouverte aux jeunes chimistes, aux physiologistes qui doivent, avant toute entreprise, acquérir une connaissance parfaite des méthodes de culture pastoriemnes des mieroorganismes.

$$
*
$$

D'où viennent ces êtres ? "Ce n'est pas sans surprise, dit Pasteur en février 1859, que l'on voit un abondant dépôt de matière végétale et animale dans une liqueur qui ne renfermait primitivement d'autre produit azoté qu'un sel d'ammoniaque. ...Quant à l'origine de la levure lactique, elle est due uniquement à l'air atmosphérique ; nous retombons ici dans les faits de générations spontanées. » 
Pouchet, professeur d'histoire naturelle à Rouen, soutenait alors, avec la majorité de ses contemporains, la possibilité d'organisation d'éléments vivants à partir de composés organiques morts, et nous verrons bientôt les phases successives des discussions soulevées à l'occasion de ce problème fondamental sur les origines de la vie. Je ne puis résister à donner auparavant une idée concrète des pensées intimes de PAsteur à cette date. Elle résulte d'une lettre de PASTEur à Pouchet, datée de l'Ecole Normale supérieure, le 28 février 1859, et publiée dans le tome II (page 628) des Euvres de l'asteur, réunies par M. Pasteur Vallery-Radot.

" J'ai vu le bacterium termo et ses diverses variétés apparaître en quantité parfois considérable dans un milieu formé... (uniquement) de matières cristallisables... L'expérience réussit très bien lors même que le vase est rempli de liquide..., e'est qu'il y a eu contact avec l'air commun pendant la préparation du milieu; mais lorsque la liqueur est prête, faites-la bouillir quelques minutes dans un ballon effilé communiquant par un caoutchoue à un petit tube de cuivre qui est entouré de charbons ardents, puis laissez refroidir, et alors fermez par un trait de chalumeau la partie effilée, puis portez le ballon dans une étuve. Il pourra y demeurer des mois entiers à une température de $25^{\circ}$ à $35^{\circ}$, sans donner aucune apparence de fermentation, ni levures, ni infusoires.

"Lorsqu'à la suite des expériences que je viens d'indiquer vos adversaires prétendent qu'il y a dans l'air les germes des productions organisées des infusions, ils vont au delà des résultats de l'expérience ; ils devraient dire simplement que dans l'air commun il y a quelque chose qui est une condition de la vie, e'est-à-dire employer un mot vague qui ne préjuge pas la question dans ce qu'elle a de plus 
délicat. Autant vaudrait dire, en effet, qu'il y a dans l'air commun de petits cristaux de Sulfate de soude, des germes de Sulfate de soude, passez-moi ces expressions, parce que cet air provoque la cristalisation d'une solution saturée de ce sel, propriété que n'a pas l'air chauffé. ") (Voir p. 97.)

"Qu'y a-t-il dans l'air qui provoque l'organisation? Sont-ce des germes ? Est-ce un corps solide ? Est-ce un gaz ? Est-ce un fluide ? Est-ce un principe tel que l'ozone ? Tout cela est inconnu et invite à l'expérience. "

La question est nettement posée. Pasteur n'a pas l'idée préconçue des germes spécifiques au début de l'année 1859 . Cette notion s'impose à lui par l'étude du problème des générations spontanées, par les ensemencements avec des ferments divers des différents milieux, par les expériences des cristallographes relatives aux eonditions de la sursaturation. Mais, au fur et à mesure de ses épreuves expérimentales, la conviction de l'absolue nécessité de germes préformés s'impose à son esprit.

Louis Pasteur présenta l'essentiel de ses découvertes sur l'impossibilité actuelle des Générations spontanées dans plusieurs communications à l'Académie des Sciences et à la Société chimique de Paris, du 9 décembre 1859 au 12 novembre 1860 ; le détail de ses études et des conceptions qui l'ont guidé est publié dans le mémoire définitif paru en 1862 (1).

Dans un historique concis, il oppose les conceptions de Needian (1745), inspirateur de Buffon, à celles de SpaLLANZANI (1765-1769), qui pose correctement le problème.

1. - Mémoire sur les corpuscules organisés qui existent dans l'atmosphère. Examen de la doctrine des générations spontanées : Ann. Chim. et Physique, 1862, $3^{\ominus}$ sér., t. LXIV, pp. 5 et suiv. 
Le prêtre eatholique NEEDHAM sontint que la matière ayant eu vie conservait après la mort une vitalité propre, sous l'influence de laquelle ses parties disjointes se réunissaient de nouveau, dans certaines conditions favorables, avec des variétés de structure et d'organisation déterminées par ces conditions mêmes. Il en donna comme preuve que des substances diverses, végétales ou animales, exposées à l'action prolongée de la température élevée dans des vases hermétiquement clos, présentaient des altérations liées à la pullulation d'êtres très variés que le microscope permettait d'isoler. NeEdham et Buffor réfutaient par là le système du pasteur suisse BonNET, qui croyait voir des mâles, des femelles, des accouplements et des œufs, dans les variations d'aspects suivis au microscope, et se trouvait le porte-parole de tous ceux qui soutenaient la persistance et la préexistence des germes.

En 1765, l'abbé Spallanzani, célèbre physiologiste italien, prend position contre la théorie de Néedham et de Buffon ; il demande que les expériences des vases clos exposés à l'action du feu soient reprises avec une précaution spéciale: "Si après avoir purgé, par le moyen du feu, et les substances que l'on met dans les vases et l'air contenu dans tes mêmes vases, on porte encore la précuution jusqu'à leur ôter toute communication avec l'air ambiant et que, malgré cela, à l'ouverture des fioles, on y trouve encore des animaux vivants, cela deviendra une forte preuve contre le système des ovaires. ")

Needram lui répond (1769) : "Qu'il se serve en renouvelant ses expériences de substances suffisamment euites pour détruire tous les prétendus germes qu'on croit attachés, ou aux substances mêmes ou anx parois intérieures, ou flottant dans l'air du vase; qu'il scelle ses rases hermétiquement 
en y laissant une certaine portion d'air sans le bouleverser ; qu'il les plonge ensuite dans l'ear bouillante..., en un mot, qu'il prenne toutes les précautions qu'il voudra pourvu qu'il ne cherche qu'à détruire les prétendus germes étrangers qui viennent du dehors, et je réponds qu'il trouvera toujours de ces êtres vitaux microscopiques en nombre suffisant pour prouver mes principes. ")

J'ai souligné les deux phrases qui précisent le débat, celles qui ont inspiré l'œuvre de Pas'reur. Gay-Lussac, guidé par la découverte de la composition de l'air par Lavoisier, affirma, en 1820, que l'absence du gaz oxygène est une condition nécessaire pour la conservation des substances animales et végétales dans les vases clos et chauffés dans l'eau bouillante pendant trois quarts d'heure; il prenait nette. ment parti pour l'explication de NeEdham. En 1837, SchwanN réalisa l'expérience réclamée par NeEdHam; une infusion de muscles est mise dans un ballon de verre dont le col est fermé par un bouchon percé de deux trous traversés par des tubes de verre coudés et recourbés, de manière que leurs courbures soient plongćes dans des bains d'alliage fusible à une température voisine de $200^{\circ}$; à l'aide d'un aspirateur, il règle le passage de l'air qui arrive froid dans le ballon; on commence par faire bouillir le liquide ; après refroidissement, on fait passer l'air calciné. Il ne se produit pas d'altérations. Pour Schwann, " ce n'est pas l'oxygène, du moins l'oxygène seul de l'air atmosphérique qui les occasionne, mais un principe renfermé dans l'air ordinaire et que la chaleur pent détruire "). 


\section{$\stackrel{* *}{* *}$}

Pasteur venait d'étudier la fermentation du lait et il avait été frappé par la petite taille du ferment lactique, cause du lait caillé. Son flair l'avertit qu'il était sur la bonne voie en ramenant toutes les fermentations et putréfactions au type de la fermentation alcoolique, incontestablement due à l'organisme levure. Il examine le problème de la génération spontanée que l'Académie venait de poser (1860) en minéralogiste et devient, par la nature même des objets examinés, un botaniste. Il veut s'assurer qu'il existe des germes dans l'air : "Le procédé que j'ai suivi consiste à filtrer un volume d'air déterminé sur du coton-poudre, soluble dans un mélange d'alcool et d'éther. Les fibres du coton arrêtent les particules solides. On traite alors le coton par son dissolvant. Après un repos suffisamment prolongé, toutes les particules solides tombent au fond de la liqueur ; on les soumet à quelques lavages, puis on les dépose sur le porteobjet de microscope, où leur étude devient facile. " Il fait agir sur elles divers réactifs, l'eau, l'iode, la potasse, l'acide sulfurique, les matières colorantes.

" Il y a constamment dans l'air commun un nombre variable de corpuscules, dont la forme et la structure annoncent qu'ils sont organisés, ...sphériques ou ovoïdes..., ...translucides ou opaques avec granulations à l'intérieur..., si divers de volume et de structure qu'ils appartiennent, sans conteste, à des espèces fort nombreuses. "PASTEUR constate aussi que l'emploi d'une seule bourre n'arrête pas toutes les poussières ; celles qui sont arrêtées par la seconde bourre ne sont plus qu'en très petit nombre. 
Y a-t-il parmi ces corpuscules organisés des germes féconds? Pour s'assurer de leur vitalité, il compare les effets de l'introduction dans une eau de levure sucrée portée à l'ébullition pendant quelques minutes, d'une faible partie des petites bourres de coton chargées de poussières. Il arrive, constamment, que des productions commencent à apparaître dans le ballon après vingt-quatre, trente-six ou quarante-huit heures au plus. Par exemple, plusieurs ballons d'eau de levure sucrée mis à l'étuve à $30^{\circ}$, dans les premiers jours de novembre 1859 jusqu'au 8 janvier 1860, sont restés inaltérés; ce jour-là, il introduit dans l'un des des ballons une portion de bourre de coton chargée de poussière. Vingt-quatre heures plus tard, rien de particulier ; neuf heures après, il voit des petites touffes soyeuses de moisissures sortir du tube aux poussières, limpidité parfaite du liquide; vingt-trois heures après, outre les touffes, il aperçoit sur les parois du ballon un grand nombre de traînées blanches irisées qui sont constituées par une Torulacée très élégante; le lendemain, le liquide, limpide jusque-là, est trouble au point qu'il n'y distingue plus les touffes de mycelium ; une foule de Bactériums, de la plus petite dimension, pirouettant ou se balançant, en est la cause. Le tube à poussière a done introduit des germes vivants de plusieurs sortes.

La démonstration paraît très simple ; elle n'a été possible qu'après la découverte pénible d'un procédé parfait de préparation des ballons stériles. L'introduction d'air calciné dans les ballons chauffés, par le moyen de la cuve à mercure, a toujours ou presque toujours échoué, parce qu'il est impossible que la manipulation, de quelque manière qu'elle soit dirigée, n'introduise pas de germes provenant de l'intérieur, ou de la surface du mercure, ou des parois de la cuve. Le procédé suivant a donné une réussite totale et constante : 
Le col effilé du ballon, de 250 à 300 centimètres cubes, renfermant 100 à 150 centimètres cubes d'eau de levure de bière et 10 à 15 grammes de sucre, est relié, comme l'indique la figure 19 , avec un tube de platine chauffé au rouge ; on
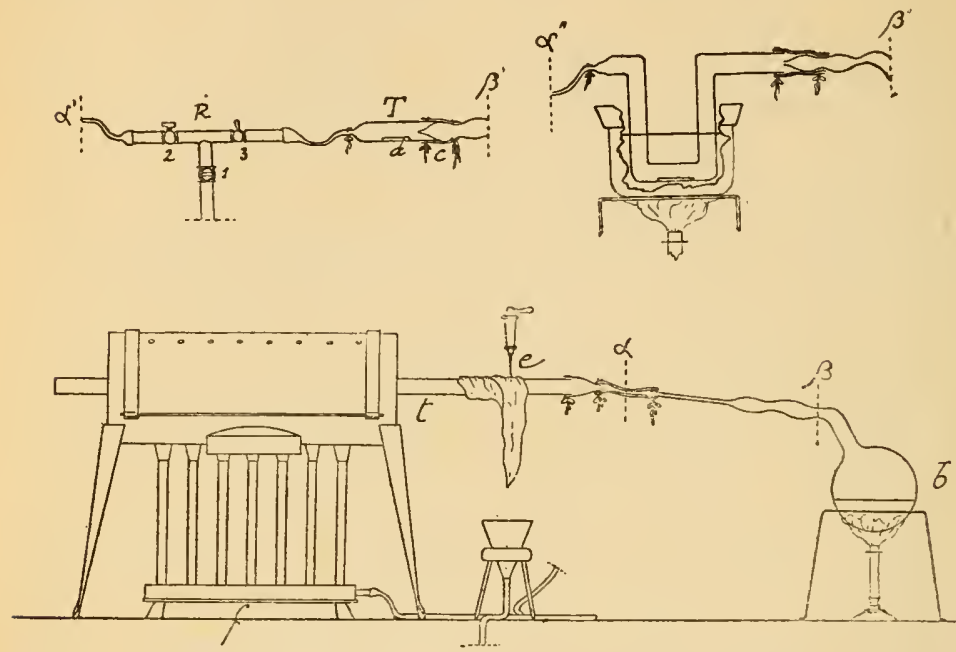

Fig. 19. - Appareil de Pastaur utilisé pour démontrer l'absence de génération spontanée; $b$, ballon à demi rempli de bouillon de culture; $f$, four à chauffer le tube de rentrée d'air $t$ refroidi en $e ; \beta$, pointe du ballon qu'il faut sceller à la fin de l'opération; $\alpha^{\prime} \beta^{\prime}$ portion de rechange pour introduire aseptiquement les poussières du tube $\alpha ; \alpha^{\prime \prime} \beta^{\prime \prime}$ portion de rechange pour introduire l'amiante, calcinćc ou non, à intercaler entre $\alpha \beta$.

fait bouillir le liquide pendant deux à trois minutes, puis on le laisse refroidir complètement ; il se remplit d'air ordinaire à la pression de l'atmosphère, mais dont toutes les parties ont été portées au rouge ; puis on ferme à la lampe le col du ballon.

" J'affirme avec la plus parfaite sincérité que jamais il 
ne m'est arrivé d'avoir une seule expérience, disposée comme je viens de le dire, qui m'ait donné.un résultat douteux. " Cette impeccabilité dans la méthode de préparation des milieux de cultures stériles est le point essentiel des démonstrations fournies par PASTEUR. Sur elle repose toutes les découvertes ultérieures.

Pour introduire le tube à poussière dans l'enceinte stérile, il fallut des précautions aussi minutieuses. Les ballons, préparés un ou deux mois d'avance, sont maintenus tout ce temps à $1^{\prime}$ 'étuve à $25^{\circ}$ ou $30^{\circ}$ et n'ont subi aucune altération sensible. "La pointe du ballon étant ferméc, on l'adapte à l'appareil (fig. 19) modifié en $\alpha \quad \beta$ par la substitution du dispositif $\alpha^{\prime} \beta^{\prime}$ : T est un tube de verre fort de diamètre intérieur 10-12 millimètres, dans lequel le tube $a$, de petit dia. mètre, renfermant la bourre de coton chargée de poussières, peut glisser facilement ; $R$ est un tube de laiton en forme de $\mathrm{T}$, muni de robinets; le robinet 1 communique avec la machine pneumatique, l'autre, 2 , avec le tube de platine chauffé au rouge ; le troisième, 3 , avec le tube $T$; $c$ représente le tube de caoutchouc, qui réunit le ballon $b$ au tube T.

Le tube de platine est porté au ronge ; on ferme 2 , on fait le vide; on ferme 1 et on ouvre 2 , de façon à laisser rentrer peu à peu de l'air calciné. Le vide et la rentrée de l'air calciné sont répétés alternativement dix à douze fois ; le petit tube $a$ se trouve ainsi rempli d'air brûlé jusque dans les moindres interstices du coton, mais il a gardé ses poussières. Ceci fait, on brise la pointe du ballon $b$ à travers le caoutchoue; on fait couler le petit tube aux poussières dans le ballon $b$, dont le col est fermé de nouveau à la lampe. Reporté à l'étuve, il donne des productions vivautes après vingt-quatre, trente-six, quarante-huit heures au plus. 
Pour plus de sûreté encore, Pasteur remplace le coton dans l'appareil à filtrer l'air par de l'amiante calcinée (fig. 19, $\alpha^{\prime \prime} \beta^{\prime \prime}$ ). Il s'assure que l'introduction d'amiante calcinée ne produit aucune altération, qu'avec l'amiante non calcinée il y a constamment des productions vivantes. " J'ai répété, conclut PaSTEUR, un grand nombre de fois, ces expériences comparatives, et j'ai toujours été surpris de leur netteté, de leur constance parfaite... Il ne m'est pas arrivé une seule fois de voir réussir les expériences à blanc, comme je n'ai jamais vu l'ensemencement des poussières ne pas fournir des productions organisées.

“ En présence de tels résultats, confirmés et agrandis par ceux des chapitres précédents, je regarde comme mathématiquement démontré que toutes les productions organisées qui se forment à l'air ordinaire dans de l'eau sucrée albu. mineuse, préalablement portée à l'ébullition, ont pour origine les particules solides qui sont en suspension dans l'air. ")

Possédant une méthode rigoureuse de démonstration, PASTEUR l'applique aussitôt au perfectionnement de sa technique, en la simplifiant, et à l'étude de plusieurs problèmes d'intérêt capital, tels que l'évaluation de la résistance des divers germes à la chaleur et la détermination des milieux nutritifs les plus favorables à chacun d'eux. Je n'insiste, pour le moment, que sur le perfectionnement et la simplification des procédés d'étude des germes de l'air.

PASTEur place dans des ballons de verre des liqueurs fort altérables à l'air, eau de levure de bière sucrée, jus de betterave, eau de poivre pilé, puis étire à la lampe les cols des ballons de manière à leur donner diverses courbures. Il porte ensuite les liquides à l'ébullition durant quelques minutes, jusqu'à ce que la vapeur d'eau sorte abondamment par l'extrémité du col effilé restée ouverte, sans autre précaution; 
il laisse ensuite refroidir le ballon. "Chose singulière, bien faite pour étonner toute personne habituée à la délicatesse des expériences relatives aux générations spontanées, le liquide de ce ballon restera indéfiniment sans altération. On peut le manier sans aucune crainte, le transporter d'un lieu à un autre, lui laisser subir toutes les variations de température des saisons, son liquide n'éprouve pas la plus légère altération et conserve son odeur et sa saveur... Que si, après un ou plusieurs mois de séjour à l'étuve, on détache le col du ballon par un trait de lime, sans toucher autrement au ballon, après vingt-quatre, trente-six et quarante-huit heures, les moisissures et les infusoires eommenceront à se montrer, absolument comme à l'ordinaire... »

Les mêmes expériences peuvent se répéter sur le lait, pourvu qu'on ait la précaution de produire l'ébullition à la température de cent et quelques degrés et de laisser le ballon se refroidir pendant qu'il y rentre de l'air calciné.

Dans les changements de température, le mouvement de l'air ne se fait sentir avec quelque intensité que dans le col et c'est là seulement qu'il peut $y$ avoir dépôt des germes que l'air transporte. On n'arrive à provoquer des productions organiques dans le liquide que par une très brusque agitation du liquide. Un autre moyen, qui réussit le plus souvent pour déterminer l'apparition des productions, consiste à fermer l'extrémité effilée du ballon aussitôt après, ou mieux, pendant l'ebullition; le vide se fait ensuite par la condensation..., l'air rentre avec force dès qu'on débouche l'extrémité, entraînant avec lui toutes les poussières jusqu'au contact du liquide.

Ces diverses expériences fournissent les meilleurs guides pour la conduite normale et régulière des stérilisations actuelles. La technique a été mise au point par PASTEUR dans 
son premier mémoire sur le sujet, et c'est certainement la raison qui lui a permis de triompher avec tant d'éclat des objections des partisans de la génération spontanée.

Je ne rappelle ici que pour mémoire les fameuses discussions avec Pouchet, avec Frémy, avec Bastian. Ce dernier montra à PAsteur que certaines de ses cultures restaient en apparence stériles parce qu'elles étaient acides ; en les rendant lègèrement alcalines, des microbes s'y développent. Pasteur reconnut le bien-fondé de cette observation, sans toutefois y voir une preuve de la génération spontanée; son élève ChamberLand s'assura qu'à 100 degrés, et en liqueur acide, les spores, découvertes par Pasteur dans le ferment butyrique (fig. 17, $b u_{i}$ ), restaient inertes, mais n'étaient pas tuées; l'addition d'un alcali leur rendait l'activité. Il faut chauffer à 120 degrés centigrades les liquides qu'on veut stériliser.

La technique même du chauffage sous pression dans la marmite de PAPIN, qui est devenue l'autoclave, ne fut perfectionnée qu'après de nombreux tâtonnements. En 1884, on reconnut la nécessité de chasser préalablement tout l'air de l'autoclave et des ballons pour obtenir une température uniforme, mesurée par le mouvement de l'aiguille sur le manomètre. Ces techniques sont depuis longtemps classiques.

Jamais, jusqu'ici, il n'a été trouvé de génération spontanée dans les liquides ni sur les milieux solides, préparés par milliards de flacons, avec les procédés convenables. La pratique industrielle de la fabrication des conserves, des laits stérilisés, donne la preuve qu'on doit admettre son impossibilité dans les conditions actuelles. 


\section{ADAPTATIONS RÉCIPROQUES DES GERMES ET PARASITES HÉRÉDITAIRES}

En novembre 1899, lorsque j'entrai à l'Ecole Normale, je fus accueilli avee la plus grande bonté par M. Gernez, maître de éonférences de chimie. C'était alors presque un vieillard, dont la parole mesurée et les mouvements étudiés imposaient au premier abord; mais le regard souriant, la mobilité et la fraîcheur d'un visage de blond du Nord atténuaient rapidement l'attitude acquise par une longue carrière dans l'enseignement. Ion grand-oncle paternel avait guidé le jeune GERNEZ, élève, puis surveillant au Collège du Quesnoy (Nord), et lui avait facilité la préparation an concours de l'Eicole Normale. M. Gernez me rappela ses souvenirs d'enfance, et je ne manquai plus aucune occasion de le rencontrer. Bien que mes études fussent, en première année, la Géométrie analytique et la Physique mathématique, bien que l'Exposition Universelle en préparation m'entraînât à de fréquentes visites qui n'étaient pas toutes des pertes de temps, j'ai eu la chance, chaque jeudi matin, de faire, sous la surveillance du collaborateur de PASTEUR, des exercices d'analyse de chimie minérale.

Sans doute, je n'ignorai pas absolument les découvertes, ni surtout les conséquences médicales des travaux de $\mathrm{P}_{\text {AS- }}$ TEUR. A Lille, où j'avais fait mes études, la renommée de l'ancien doyen de la Faculté des Sciences avait traversé les murs du sombre Lycée, peut-être moins cependant que les 
échos d'une affaire célèbre. Mon ignorance était telle, lorsque Gernez me parla de son Maître, que j'en fus honteux et que je me procurai chez un bouquiniste, le lendemain, le merveilleux ouvrage de Duclaux, Histoire d'un Esprit. Je le lus avec avidité, comme un roman, et ne manquai pas d'en parler à Gernez, qui m'écouta avec plaisir ; mais, avec une certaine malice, il m'engagea à lire les œuvres de PASTEUR lui-même et, comme je le regardai étonné : "Lisez les Etudes sur la maladie du Ver à soie, par exemple. »

A mon avis, cette lecture est la meilleure introduction, pour un étudiant peu familiarisé avec la cristallographie et la chimie complexe des fermentations, dans l'analyse des méthodes, du raisonnement et de la perfection technique qui caractérisent les travaux de Pasteur. Peut-être Gernez, qui se rendait bien compte qu'une partie de son mérite personnel était clairement mise en évidence dans cette ouvre, n'a pas donné le conseil sans une pointe d'amour-propre; mais je maintiens qu'il n'y a guère d'ouvrage, trop rare malheureusement, même dans les meilleures bibliothèques, qui soit plus désigné pour frapper l'imagination et fournir une idée des difficultés, des joies aussi, de la recherche biologique.

A la même époque je lisais, dans la Revue générale des Sciences (1900), dont le service était fait gratuitement aux Normaliens, un article de M. J. ERIKsson, le savant suédois, sur les races physiologiques des Rouilles des céréales. Mon père, agriculteur passionné, me demandait de le tenir au courant des progrès de la lutte contre les maladies des plantes cultivées. Je ne crois pas qu'au cours de cette première année d'Ecole, sauf peut-être aux leçons de Houssay et de Lippmann, j'aie éprouvé des impressions plus vives que celles qui résultèrent de la lecture presque simultanée 
des Etudes sur la maladie du Ver à soie et de l'adaptation si remarquable des Rouilles parasites à leurs hôtes. Je désire vous montrer le lien qui les unit dans ma pensée.

A la fin de juin, en certaines années, les champs de blé d'un village, d'un canton, prennent une couleur orangée, présage d'une mauvaise récolte ; les sarcleuses sortent des champs couvertes d'une poussière rouille, qui se détache des feuilles et des chaumes. Tulasse a décrit avec soin l'évolution $d u$ Champignon parasite, connu sous le nom de Rouille orangée du Blé ou Puccinia graminis; on sait deprais que les spores ovales, uredo, tombent sur les feuilles humides des céréales, germent en poussant des tubes grêles qui, rencontrant un stomate, pénètrent à l'intérieur des tissus, y accumulent leurs filaments ; ils ne tardent pas à y organiser des corbeilles couvertes de tubes parallèles formant des faisceaux dressés et terminés bientôt par les mêmes spores ovales, qui font éclater l'épiderme de la de la feuille par plages allongées linéaires, d'où le nom d'Uredo linearis donné à cette phase de croissance du parasite. Les spores s'échappent de nouveau et multiplient la maladie au point que toutes les feuilles, tous les chaumes, et jusqu'aux balles des épis, en sont couverts lorsque les orages répétés, la chaleur et l'humidité, favorisent l'extension de l'épidémie.

Un mois plus tard, peu de jours avant la moisson, les taches changent de teinte, deviennent plus foncées, puis virent au noir. Les urédospores ont presque totalement disparu pour faire place à d'autres organes reproducteurs, les téleutospores, d'aspect très différent, formés essentiellement de deux cellules oblongues, accolées en fuseau et portées sur des pédicelles relativement longs; pédicelles et cellules se 
détachent difficilement au cours de la fauche ou de la récolte des blés; elles tombent sur le sol, passent l'hiver à l'état de vie ralentie; au printemps, elles germent en donnant deux courts filaments, qui portent bientôt chacun quatre petites cornes qui s'isolent et constituent les basidiospores. Le vent les emporte sur les Graminées, sur les mauvaises herbes, sur les haies.

Un premier fait me frappa, très clairement expliqué par M. Eriksson. Jamais une basidiospore ne germe sur le Blé ni sur aucune des Graminées voisines ; transportée par le vent sur une feuille d'Epine-vinette (Berberis vulgaris). elle s'y fixe, donne un filament qui ne tarde pas à pénétrer dans les tissus; elle y forme des taches épaisses et à contours arrondis où les filaments s'organisent en bouteilles, dont l'intérieur est rempli de spores d'une quatrième sorte, les aecidiospores, pulvérulentes, anguleuses, qui, mises à leur tour en liberté, tombent sur les feuilles du Blé et y déterminent la Rouille à urédospores ; en dix jours, la fructification développée sur l'Epine-vinette reproduit la fructification propre au Blé.

Il y a de nombreux exemples de ces alternances de formes du même parasite, avec multiplication à l'extrême des germes susceptibles d'étendre la contamination. L'histoire des migrations des Vers plats parasites est exposée dans le cours préparatoire au baccalauréat, et j'en avais conservé le souvenir. La spécialisation si marquée de la Puccinie du Blé n'attaquant que l'Epine-vinette, me parut plus remarquable ; mais je doutai, comme les praticiens et les savants de cette époque, que ce fût le seul mode de propagation de la Roupille des Céréales, si commune dans certaines années üans les plaines des Flandres, où l'Epine-vinette est très rare. 
La découverte de M. ERIKsson qui me frappa le plus fut l'étroite spécialisation des Rouilles avec leurs hôtes. Alors qu'avant 1894 on ne connaissait que trois espèces de Rouilles attaquant les Céréales (Puccinia graminis, P. rubigo-vera et F. coronata), le biologiste suédois s'assura par des inoculations expérimentales qu'en Suède la première présente au moins sept formes, la seconde huit formes, la troisième onze formes.

Les formes qui se rapportent à une même espèce ne montrent entre elles aucune différence morphologique, quant à l'aspect extérieur et à la structure interne microscopique ; l'ornementation et la taille des spores, qui est d'ordinaire le critérium distinctif le plus sûr, ne permettent pas de les séparer. Mais chaque forme est spécialisée à l'espèce de Céréale sur laquelle on la découvre; elle ne peut être inoculée qu'à cette Céréale.

Ainsi, une paille de Seigle attaquée par la Rouille brune peut communiquer la maladie au Seigle, mais non au Froment; une paille d'Avoine attaquée par la Rouille noire ( $P$. graminis) peut contaminer les chaumes et les feuilles d'une Avoine saine, mais son parasite ne pent vivre dans les tissus du Seigle, de l'Orge ou du Froment. On ne trouve des exceptions à la règle de spécialisation que très rarement ; la variété $P$. graminis $\mathrm{f}$. secalis prospère à la fois sur le Seigle et l'Escourgeon. Il y a done autant de formes physiologiquement indépendantes qu'il y a d'hôtes spécifiquement distincts.

La découverte des formes spécialisées de Rouilles résulta des essais d'inoculation des spores de multiplication de printemps du type uredo. M. Eriksson s'assura ensuite par une série parallèle d'épreuves d'inoculations que, même après son passage sur l'Epine-vinette, la forme de Rouille propre 
au Seigle donnait des aecidiospores dont les sporidies étaient dangereuses uniquement pour le Seigle, absolument inoffensives pour le Blé. Cette découverte a une grande importance pratique; elle justifie la pratique ordinaire des rotations de Céréales, de la culture de l'Avoine après Blé, de l'Orge après Avoine, sans avoir à tenir compte des épidémies de Rouille présentées par l'une ou l'autre espèce de Céréale. Ces faits ont été vérifiés depuis par de nombreux expérimentateurs.

M. Eriksson essaie alors (1900) de démontrer que la propagation des Rouilles spécialisées se fait par les semences des céréales. Il déduit d'expériences en chambres stérilisées et d'observations microcospiques que le plasma de la Rouille propre à une espèce est renfermé à l'état latent dans l'embryon ; qu'il s'y trouve entraîné sous une forme mycoplasmatique mal définie; qu'il prend au cours de la croissance de l'hôte tous ses caractères propres, filaments, nature des pustules et des spores, aptitudes physiologiques à la contamination. L'existence d'un mycoplasma héréditaire dans les semences des Céréales n'est pas actuellement démontrée et quelques faits rendent douteuse son existence à l'état d'indifférentiation. Mais cette affirmation cadrait si exactement avec les observations de PAsteUn, que mon imagination fut frappée par ce rapprochement.

$$
\text { ** }
$$

Revenons à la lecture des Etudes sur la maladie du Ver à soie $(1870)$. PASTEUR expose avec détails les caractères constants et les symptômes accidentels de la maladie et, comme il l'a fait pour la découverte de l'hémiédrie des tartrates, cherche à distinguer ce qui est fondamental des 
particularités accessoires. Discutant après vérification les deseriptions de son collègue naturaliste, DE QUATEFAGES, il dit dans le préambule :

"Une seule maladie, celle des morts flats..., est associée à la pébrine, mais sans relation nécessaire avec cette dernière, ce dont je ne veux d'autre preuve en ce moment que le fait bien avéré de l'absence complète des morts flats dans les localités où sévit la pébrine au plus haut degré. Je montrerai d'autre part que les taches de la peau des vers malades ne sont pas l'effet d'une gangrène, mais bien d'un parasite intérieur, introduit dans le ver par hérédité on par contagion. ")

La maladie des Vers à soie avait en effet été décrite dans les Mémoires
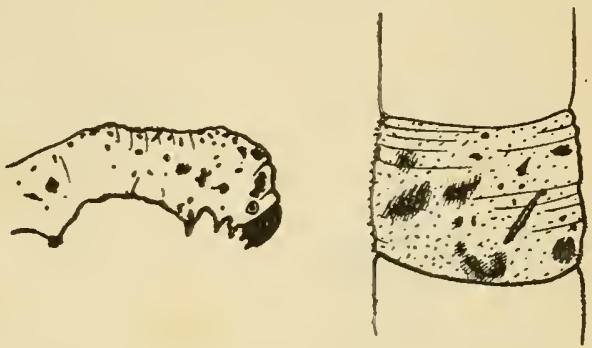

Fig. 20. - Partie antérieure du corps et anneau d'un Ver pébriné (d'après Pasteur).

de l'Académie des Sciences (1858-59) par DE Quatrefages comme une dégénérescence complexe, de l'ordre des putréfactions. Le médecin pouvait croire avoir sous les yeux une affection gangréneuse viciant l'organisme dans ses plus intimes profondeurs, tout en produisant parfois des plénomènes que l'on rapporte an rachitisme. A la surface de la peau du Ver, il constatait des taches noires plus on moins nombreuses, les unes à bords tranchés et nets, les autres auréolées et de teinte jaunâtre, d'où le nom de pébrine rappelant l'aspect du poivre pilé (fig. 20).

Une autre maladie, la Muscardine, due au développement du champignon Botrytis ou Isaria, compliquait aussi les 
symptômes et, dès 1849, un naturaliste français, GuḱRINMenseville, avait voulu en trouver l'origine dans des éléments ovalaires, réniformes, de même grosseur (1 $1 / 400$ de millimètre) présents d'ordinaire dans le sang des Vers et dispersés au milieu des globules propres du sang beaucoup plus volumineux. On avait d'ailleurs constaté que plus les Vers étaient malades, moins il y avait dans le sang de globules nolmaux et plus nombreux étaient les corpuscules décrits par Guérin ; mais, en même temps, on s était assuré qu'il n'y
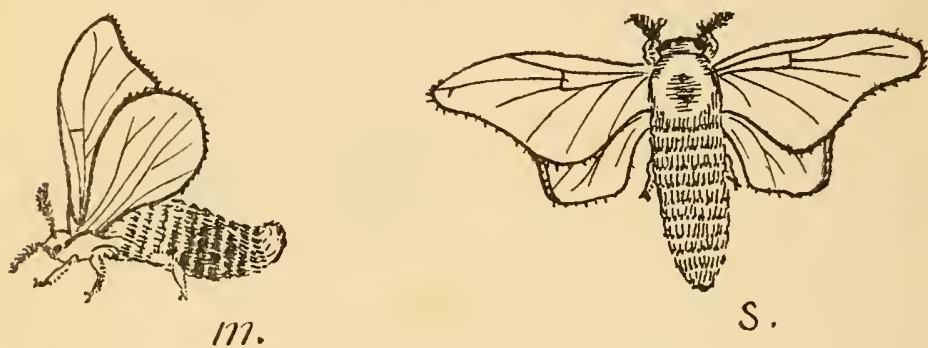

Fig. 21. - Papillons du Ver à Soie; en $m$, malade avec anneaux de l'abdomen bruns; en $s$, sain (d'après Pastbur).

avait aucune relation entre les corpuscules et le Champignon filamenteux, cause de la Muscardine.

PAsteur écarte résolument cette dernière cause d'erreur, en faisant remarquer que la maladie comnue sous le nom de Muscardine n'a jamais moins sévi que depuis la vérification presque constante de la présence desdits corpuscules dans le corps des Vers. Il rend hommage au savant italien FiLIPPI qui, dès 1850, avait signalé l'erreur de GuÉRIN-MENNEviLLE, mais il s'écarte des conclusions données par FILIPPI, qui admet que les corpuscules sont une formation normale de l'organisme des Vers sains. Au milieu des centaines de publications qui ont été faites sur le sujet, PASTEUR suit pas à pas 
les progrès de l'observation de ces corpuseules : Connalia (1856), Lebert et Frey (1856 et 1858), établissent une relation probable entre la fréquence des corpuscules et les symptômes graves de la maladie. Le papillon femelle surtout paraît malade; il présente des taches gris de plomb sur les anneaux de l'abdomen et sur les ailes, qui sont parfois fripées (fig. 21) : sa ponte est faible et difficile. Le sang est alors très riche en corpuscules vibrants et noircit, mais pas tonjours, la lamelle du microscope qui permet de les examiner. En 1857, le docteur Osimo, de Padone, reconnaît même la présence de corpuseules dans l'œuf ou graine du Ver à soie, et, en 1859, Carlo Vittani constate que la proportion des wufs visiblement corpusculeux augmente au fur et à mesure de l'approche de l'éclosion. Bref, Cornaliı décrit une méthode de préparation de la bonne graine, utiliśée en Italie, puis en France, en 1860 et 1861, qui consiste à cxaminer les œufs par transparence, à rejeter les lots où le microscope met en évidence les corpuscules dans les cufs, ou simplement en contact superficiel avec les œufs, et à conserver les lots de graines constamment privées de corpuscules.

Pasteur était familiarisé plus que quiconque avec ce genre de travail ; il remarcuue que la méthode de triage de la bonne graine parmi les mauvaises a un vice radical, e'est qu'une graine faite est toujours élevée. Et, le 26 juin 1865, avec une intuition qui ne peut dériver que de sa conviction intime que les germes n'apparaissent pas spontanément, mais se transmettent sans se détruire sous des formes diverses, PASTEUr affirme qu'il " devait y avoir un moyen infaillible de se procurer une graine saine, en ayant recours à des papillons exempts de corpuscules. ") 
E. Duclaux, puis MI. R. Vallery-Radot, nous rappellent les circonstances tragiques que traversait PASTEUR au moment où le ministre DuMas le chargea d'une mission à Alais, le 6 juin 1865, pour étudier les causes et limiter les ravages de la maladie du Ver à soie. PASTEUr ignorait tont de l'élevage du précieux insecte et, dès les premiers jours, il se familiarisa avec les travaux de la chambrée et aussi avee la certitude décevante que dans les lots en apparence prospères le corpuscule n'était pas l'exception, mais la règle. Neuf jours après son arrivée, il fut appelé à Arbois auprès de son père mourant ; en septembre de la même année, il perdit sa fille Camille, gravement malade depuis plusieurs mois.

Mais Pasteur était avant tout l'homme du devoir; il ne pouvait laisser ignorer les premiers résultats acquis; maîtrisant sa douleur, le 26 juin, au Comice agricole d'Alais, il prononçait son diagnostic ; il avait constaté que, si les vers corpusculeux à leur dernière période filaient des cocons parfaits, leurs chrysalides, en pleine maturité, à la veille de devenir papillons, étaient remplies de corpusenles; quant aux papillons, nul n'en était exempt. Le moyen infaillible de se procurer une graine saine était done bien d'avoir recours à des pontes provenant de papillons exempts de corpuscules.

Il donne à l'Académie des Sciences la même année (15 septembre 1865) l'explication de la valeur de son procédé : “ N'est-il pas logique d'admettre que le Ver sera d'autant plus malade à l'origine et plus éloigné de pouvoir monter à la bruyère qu'il proviendra d'une graine issuc de parents plus chargés de corpuscules au moment de la fonction de reproduction ? En dehors du raisonnement, tous les faits m'avaient paru conduire à cette manière de voir, et j'arrivai ainsi à 
penser que la maladie devait être regardée comme affectant de préférence la chrysalide et le papillon, qu'en d'autres termes c'est à cet âge de l'animal qu'elle se manifeste plus apparente et sans doute aussi plus dangereuse pour la postérité. »

Il s'agissait de sauver une industrie prospère et, sans hésiter, bien qu'il fût convaincu que la question présentait encore maints points obscurs, il énonça les règles du grainage cellulaire, qui donneront une sécurité aux éleveurs et limiteront l'épidémie :

"Les corpuscules peuvent être considérés comme le critérium de la maladie de l'insecte;

"Il ne faut considérer comme graine pure que celle qui est née de parents privés de corpuscules et appliquer pour se la procurer le moyen suivant :

"Isoler, au moment du grainage, chaque couple de Papillons mâle et femelle. Après le désacconplement, la femelle, mise à part, pondra ses graines; on l'ouvrira, ainsi que le mâle, afin d'y rechercher les corpuscules. S'ils y sont absents et également dans le mâle, cette graine sera conservée comme absolument pure et élevée l'année suivante avec des soins particuliers " (fig. 22).

Le procédé n'est autre que celui de la sélection généalogique, pratiquée chez les éleveurs anglais sous le nom de pedigrée. Elle suppose, et c'est ce que Pasteur va rechercher avec soin, que les ascendants transmettent en totalité ou en partie des tares, des aptitudes anssi qui s'accumulent lorsqu'on a soin de réaliser des unions aussi consanguines que possible.

Les sélectionneurs anglais $d u$ xvIII $^{\mathrm{e}}$ siècle en ont tiré le meilleur parti pour la sélection de leurs races de bovidés. 
Les Durhams de Collws avaient aequis par la consanguinité répétée une aptitude particulière à la précocité et à la régularité des formes qui firent attribuer à certains tau-

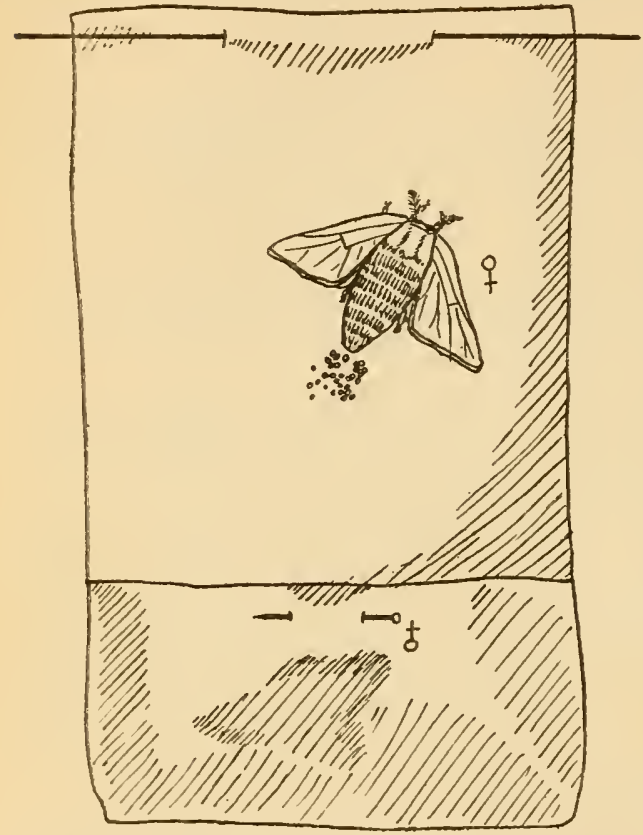

Fig. 22. - Ponte prélevée selon la recommandation de Pasteur ; la toile est repliće en sachet en bas et renferme le cadavredu papillon mâle; la femelle pond et à la fin de la ponte sera jointe au mâle pour les observations microscopiques ultérieures (d'après Pasteun). reaux, célèbres

par leur généalogie, cent fois la valeur d'une bête ordinaire de même poids. Les semences de Blé et d'Avoine, préparées an milieu du $\operatorname{xix}^{-\theta}$ siècle par le major $\mathrm{H}_{\mathrm{AL}}$ LET, eurent une réputation mondiale, et, dans les expositions agricoles, les produits figuraient constamment accompagnés d'une notice rappelant leurs origines et leur ascendance immédiate.

Pour Pasteur, l'origine n'avait d'intérêt que si elle était accompagnée de l'absence de corpuscules. Les enquêtes faites sur les principaux points de l'élevage du Ver à soie en France lui fournirent bientôt la conviction que la grande majorité des papillons étaient partout plus ou moins atteints. 
Une graine de Sauve fut cependant distinguée des autres et servit à la découverte d'une maladie différente, souvent superposée à celle des corpuscules, je veux dire la flacherie. D'autre part, les graines italiennes, les graines japonaises, étaient d'ordinaire plus on moins affectées par' les corpuscules et leur introduction, que certains préconisaient, n'offrait aucune sécurité. L'examen de papillons desséchés d'une collection d'étude, réunie avant 1850 au Conservatoire national des Arts et Métiers, prouva que la maladie des corpuscules était répandue dans tous les pays et depuis longtemps; le grainage cellulaire seul pouvait permettre la régénération des meilleures races.

L'effort de PASTEUr porta d'abord presque uniquement sur la découverte de pontes dérivées de parents non corpusculeux. Il s'assura qu'elles pouvaient provenir de deux sources : " les unes de papillons non corpusculeux, les autres de papillons qui le sont à un degré plus ou moins marqué : $1^{\circ}$ des papillons peuvent être corpusculeux et leurs œufs ne l'être pas ; $2^{\circ}$ le nombre des œufs corpuseuleux augmente généralement avec l'abondance plus ou moins grande des corpuscules dans les papillons pour une éducation déterminée; $3^{\circ}$ les graines jaunâtres, brunes, déprimées, de mauvais aspect, renferment beaucoup plus de corpuscules que les graines de belle apparence..... On ne peut douter que des œufs non corpusculeux issus de parents chargés de corpuseules soient, toutes choses égales, inférieurs en qualité à des œufs non corpusculeux qui proviennent de parents sains... les vers issus des premiers et qui ne sont pas corpusculeux n'ont pas du tout, en général, la vigueur des très bons vers nés de parents sains. " (Etudes sur la maladie du Ver à soie, page 65.) 
Et, un peu plus loin : "Toutes les fois que les corpuseules auront apparu visiblement déjà dans les chrysalides jeunes, les œufs des papillons correspondants seront corpusculeux en plus ou moins grand nombre. Au contraire, ils le scront peu, ou pas du tout, si les corpuscules ne se montrent que dans la chrysalide très âgée ou dans le papillon. La raison en est simple ; on sait que le contenu de la chrysalide est eomme un nouvel œuf pour la formation de tous les liquides et tissus du papillon. Lorsque, en même temps que se déve. loppent ces tissus, les corpuscules commencent déjà à se multiplier, on comprend que quelques-uns de ceux-ci puissent prendre naissance dans les matières de l'oviducte servant à la formation des wufs et se trouvent par conséquent à l'intérieur de ceux-ci lorsqu'ils sont pondus. Mais si les corpuseules n'apparaissent qu'en nombre plus ou moins grand dans le corps de la chrysalide et postérieurement à la formation et à la consolidation des œufs, ces derniers ne peuvent contenir le petit organisme. "

$$
* *
$$

PASTEur a rédigé cette phrase en 1870 ; il n'aurait pas écrit le mot " petit organisme " en 1866. Car, si nous sommes familiarisés actuellement avec l'idée que des organismes vivants parasites peuvent vivre, s'enkyster et même pulluler à l'intérieur des tissus d'autres êtres, nous le devons à PASTEUr et à des découvertes postérieures aux études sur les Vers à soie. Duclaux insiste avec raison (Histoire d'un Esprit, p. 199) sur cette attitude de son Maître et déclare que PASTEUr n'acceptait qu'avec répugnance l'idée que le corpuscule fût un être vivant. On peut en cffet examincr les textes avec soin ; toujours, avant 1S67, 
ils peuvent parfaitement s'interpréter avec la notion que le corpuscule est un corps inerte, résidu du fonctionnement de l'organisme, mais qui ne s'y dépose que s'il y a un germe, à la façon des germes cristallins dans les solutions sursaturées de Gernez et de Violette. La répartition des germes; immobiles par eux-mêmes, dans la masse fluide des tissus de la chrysalide sur le point de se métamorphoser en papillon, était done purement accidentelle, et PASTEUR en conclut, avec apparence de raison, qu'il était possible de trouver, par des recherches patientes dans toutes les races de papillons, quelques reproducteurs dont les œufs seraient formés sans inclusions de ces germes microscopiqnes. En fait, i! souligna, par une erreur d'interprétation il est vrai, l'inutilité qu'il y a à rejeter, dans la lutte contre les maladies causées par des êtres organisés ou non, tous les individus suspects, et de détruire, comme on l'a proposé quelquefois, des races d'élite que l'on pourrait sauver avec quelques précautions.

En 1866, Pasteur fit une première épreuve, d'ailleurs non suivie de succès, de contamination par ingestion de corpuscules de quelques vers sains que lui remit, à Paris, son collègue de l'Institut, Peligot ; les Vers ne parurent pas en être affectés. A la même date, GERnEz, qui avait accompagné PASTEUR à Alais et supposait que celui-ci était convaincu de la nature contagieuse de la maladie, lui fit part de ses expériences à Valenciennes. Il y élevait une petite quantité d'une graine japonaise saine provenant d'un don que le Taïcoun avait fait à l'Empereur. Le 31 août 1866, Gernez vérifia gue les papillons issus étaient sains ; la race étant bivoltine, elle fournit deux semaines plus tard un lot de jeunes vers qui, sur la demande de 
Pasteur, fut divisé en quatre parties de quarante vers et traité de diverses façons :

$1^{\circ}$ Lime nourrie avec dos feuilles ordinaires; les vingt-sept vers qui purent être élevés allèrent jusqu'au cocon et les papillons issus furent tous exempts de corpuscules;

$2^{\circ}$ La seconde fut nourrie avec des feuilles mouillées par l'eau de lavage de papillons non corpusculeux; elle ne donna que dix-neuf cocons, tous exempts encore de corpusenles ;

$3^{\circ}$ La troisième, nourrie après la troisième mue avec des feuilles mouillées par l'eau de lavage de papillons corpusculeux, ne fournit que quatre cocons renfermant un ver mort, deux chrysalides mortes, enfin un papillon, tous corpusculeux ;

$4^{\circ}$ La dernière, nourrie après la quatrième mue seulement avec des fenilles corpusculeuses, domna vingt-deux cocons, à mortalité beancoup moindre, mais tous les papillons éclos étaient corpusculeux.

Il ne pouvait $y$ avoir de doute pour Gernez, pour Ductuux ; la contagion était certaine et le corpuscule était un être vivant. PAsteur ne s'incline pas; le 12 janvier 1867, il affirme, dans une Note présentée à la Commission impériale de Sériculture, " que la maladie est sûrement constitutionnelle dans un grand nombre de cas et précède l'apparition du corpuscule..., qu'il n'a pu surprendre jusqu'à présent un mode de reproduction du corpuscule; que son mode d'apparition le fait ressembler à un produit de la transformation des tissus ». Au printemps de 1867, il répète en grand l'expérience de GERNEz et, enfin, reconnaît franchement la nature parasitaire du corpuseule. Ce n'est plus un symptôme constant de la maladie du Ver à soie ; c'est la cause immédiate de la maladie. 
Par la même adhésion aux conclusions de Gernez, PAsTEUR admet et développera ensuite la notion de l'hérédité des complexes vivants. L'œuf du Ver à soie avee son corpuscule forme un organisme nouveau malade, qui peut se propager au moins poux une génération avec ses particularités propres. Le plus souvent, le produit affecté de bonne heure par le parasite meurt avant d'atteindre l'état adulte ; parfois il donne naissance à des ovules sains, par suite de circonstances accidentelles limitant la multiplication du parasite dans les ovaires; la descendance de l'œuf renfermant le corpuscule présentera tous les caractères héréditaires de certaines variétés instables.

$$
\text { *** }
$$

Quelques variétés de plantes à feuillage panaché ont probablement une origine parasitaire. Le fait paraît rigoureusement établi pour celles qui transmettent leurs caractères aux sujets verts sur lesquels on les greffe. Ainsi, les Abutilons panachés, si couramment employés pour la décoration des plates-bandes, se multiplient d'ordinaire par boutures et reproduisent régulièrement les macules jaunes et blanches diversement réparties sur les limbes des feuilles. On en possède des lignées, comparables en quelque mesure aux lignées pures de microbes, qui ne présentent la panachure que sur la frange des fenilles, d'autres dans les angles des denticulations; les plus communes sont à macules dispersées sur toute la surface des limbes et jẹ ne parlerai ici que de ces dernières.

Il existe aussi des lignées d'Abutilons à feuillage constamment vert, et celles qu'on obtient par le semis offrent toujours ce caractère. Sur des Abutilons verts, greffons en 
placage un bourgeon d'Abutilon à feuilles maculées; le bourgeon se développe naturellement en bourgeon ne portant que des feuilles maculées ; mais, de plus, après quelques semaines de croissance, on voit les jeunes bourgeons développés sur les rameaux propres au sujet, qui jusque-là ne donnaient que des feuilles vertes, porter à leur tour des feuilles maculées de jaune et de blane, à l'image des feuilles du greffon. Il semble qu'il y ait contagion à distance, du greffon au sujet; car, jusqu'à présent, il n'a pas été possible de mettre en évidence un parasite. Le suc cellulaire des tissus panachés possède la même action que le bourgeon greffé, mais peu dnarable. Bref, il passe quelque produit de la pousse panachée greffée aux bourgeons jeunes en voie de formation du sujet, mais il est impossible de dire si c'est un microbe invisible ou une toxine, agissant à distance et susceptible à très faible dose de limiter le dévelop. pement local des leucites chlorophylliens.

Les graines des Abutilons panachés ne reproduisent pas la panachure; mais diverses espèces de plantes très différentes sont connues pour donner régulièrement par le semis les caractères de stries ou de nervations ou de plages jaunes et blanches qui attirent l'attention sur les parents. Les Maïs et les Houblons japonais, la Barbarée vulgaire, possèdent ce caractère à un tel degré que les lignées panachées se maintiennent fixes sans soins particuliers. Chez les Pelargonium zonale, les Belles-de-Jour, les Mufliers, les Primevères de Chine, l'hérédité de la panachure des feuilles est irrégnlière; certaines lignées diffèrent des autres par leurs aptitudes, et on soupȩonne, à l'origine de ces variétés, des associations hybrides de lignées à feuillage vert et à feuillage panaché, les tissus spécifiquement distincts se séparant 
les uns des autres au fur et à mesure du développement des pousses, surtout dans les fleurs. Il arrive d'ailleurs, dans les expériences de Correns, que la fécondation du Mirabilis Jalapa albomaculata par le pollen de $M$. Jalapa vert donne uniquement des individus verts, alors que l'inverse se produit avec les Mufliers et les Pélargoniums.

J'ai obtenu, dans mes études sur le Maïs, des lignées riches en plantes panachées et en plantules albinos, ces dernières ne développant qu'un petit nombre de feuilles complètement blanches avant de mourir. Miles (1915), qui a croisé le Maïs ordinaire et le Maïs japonais panaché, affirme la transmission irrégulière des caractères des deux parents à leur progéniture et décrit des différences constantes dans la taille des cellules des parties blanches et des parties jaunes. Reste à savoir si ces différences sont spécifiques et non dues à des modifications locales de nutrition par la réduction ou la disparition des chloroleucites assimilateurs.

Quoi qu'il en soit, les problèmes de la transmission de la panachure offrent ce point commun avec les problèmes étudiés par Pasteur à propos de la Pébrine du Ver à soie que les efiets peuvent être identiques, qu'il y ait propagation de virus vivants par contagion et localisation irrégulière sur certains organes ou portions d'organes, ou simpleplement altération locale, et irrégulière elle aussi, des chloroleucites qui se multiplient par simple division dans les cellules en voie de formation. Pour affirmer la nature vivante des éléments qui causent les macules des feuilles, il faut et il suffit de s'assurer de la contagion ; des expériences, basées sur quelques faits découverts accidentellements, sont entreprises sur ce sujet par un de mes élèves. 
Les publications récentes de MI. ERwin Suntu sur les causes et la propagation du Cancer des Chysanthèmes (1912-1920) ont apporté quelques faits nouveaux relatifs à la transfusion à distance de certaines tumeurs (fig. 23), greffées en des points précis d'une bouture de Chrysantheroum frutescens. Ce savant a isolé des cellules diffuses et des
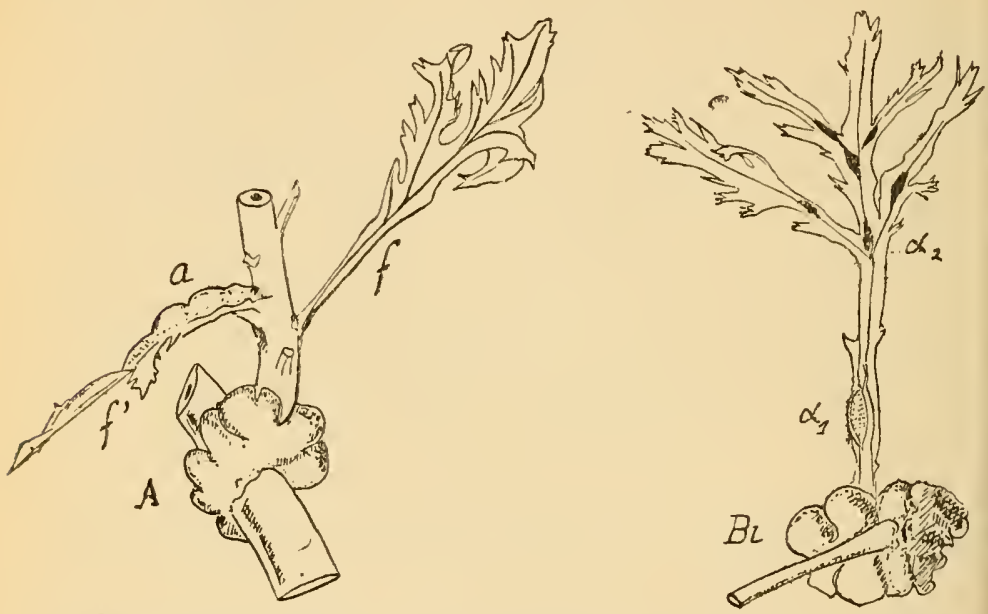

Fig. 23. - Tumeurs cancéreuses (Crown-gall) expérimentales du Chrysantheme: $A$, point d'inoculation du Bacillus tumefaciens et tumeur jeune ; $f$, feuille inférieure indemne, $f^{\prime}$ feuille déformée en $a$ avec succession de tumeurs très actives; $B i$, autre exemple de vieille tumeur au point d'infection, feuille tardive avec tumeurs ouverte en $\alpha_{1}$, encore fermée en $\alpha_{2}$; on suit la contagion le long des nervures principales (d'après Erwin Smith).

jeunes tumeurs un Bacterium tumefaciens qui est mobile grâce à des flagelles polaires et paraît localisé dans certaines cellules ou en certains points, par la réaction devenue franchement acide des cellules voisines. Les inoculations daus les tissus profond's du cambium sont suivies de proliférations cellulaires non seulement aux points d'infection, mais à plusieurs décimètres de distance, les massifs étant reliés 
entre eux par des cordons de cellules rares où des colorants électifs mettent en évidence quelques Bactéries. Les inoculations superficielles daus les tissus très jeunes de la tige déterminent des tumeurs superficielles, à croissance limitée, qui s'isolent, mais où apparaissent des groupements de cellules embryonnaires et même des vaisseanx qui ne se forment pas d'ordinaire dans l'écorce; ces vaisseaux ne se racondent d'ailleurs pas au système vasculaire normal. Il y a formation de bourgcons adventifs dans l'écorce, alors qu'elle est, d'ordinaire, limitée au cylindre central.

Tais le rôle même de ces parasites est discuté. MM. Levin et M. Levine constatent qu'en inoculant de la même façon, en des points homologues, deux plantes aussi identiques que possible, dans bien des cas la réaction se traduit, chez l'un, par une petite excroissance bénigne, chez l'autre, par une grosse tumeur maligne. Il y a des résistances individuelles notables, et ce que l'on peut affirmer, e'est qu'aujourd'hui la plupart des savants qui ont étudié le Cancer n'admettent pas l'intervention nécessaire de parasites, figurés ou non.

Il serait bon, dans ces recherches si importantes, d'imiter la prudence de PASTEUR. Les Etudes sur la maladie du Ver ì Soie sont un modèle que le Maître se plaisait à rappeler à ses élèves, à ses émules, et même à ses contradicteurs, lorsqu'il s'efforçait de préciser les phénomènes de la contagion et de la résistance, la superposition de plusieurs maladies, les relations mêmes de ces maladies entre elles.

$$
*
$$

Les discussions actuelles sur l'origine parasitaire des Crown-galls, sur le mode d'infiltration et les particularités locales de la résistance des végétaux an Bacterium tumefaciens fournissent des notions relativement claires sur le 
méeanisme de la transmission des corpuseules chez les Vers à soie. Le fait que la durée d'incubation est variable selon les animaux, selon le degré de eontamination, explique que certains ovaires, ou même des portions d'ovaires de papillons malades soient atteints, alors que les portions voisines restent indemnes; il y a, au moment où se fait cette association, une lutte intime entre les tissus sains et les tissus remplis de eorpuseules, lutte trahie par la lenteur de développement, la répugnanee à prendre la nourriture, l'irrégularité des chenilles à monter à la bruyère. PASTEUR recommande de rejeter la graine de ees lots, qui sont plus sensibles que les autres à une autre maladie : la flacherie.

En 1867, au cours d'un élevage préeoce de seize pontes provenant de parents non corpuseuleux, PASTEUR en étrudie une qui fut complètement perdue en quelques jours, à la quatrième mue; les cadavres et les malades ne renferment pas de traces de corpuseules, mais des quantités inouies de petits vibrions qui définisseut une maladie d'origine mierobienne, différente de la pébrine. "Sur le point prineipal de l'indépendance de la flaeherie et de la pébrine, l'incertitude n'était plus possible. Car les éducations atteintes, même au plus haut degré, par la maladie des morts flats sans la moindre apparence de pébrine étaient propres à des graines nées de parents exempts de corpuscules et qui eonduisaient à des reproducteurs également privés de eet organisme. Si l'on pouvait à la rigueur eonjecturer que la flacherie était une conséquence de l'affaiblissement graduel des races sous l'influence de la pébrine, il était, dans tous les cas, impossible de mettre en doute une indépendance de fait entre les deux affections. ”

La flacherie n'est, en réalité, qu'une indigestion mortelle causée par des fermentations. En trente jours d'existence, 
la chenille absorbe en matières alimentaires dix mille fois le poids de l'œuf dont elle provient ; le canal intestinal des Vers flats se comporte vis-à-vis des vibrions en bâtonnets ou en chapelets, caractéristiques de la flacherie, exactement comme un tube de verre où l'on introduirait des feuilles de mûrier broyées. La maladie est d'ailleurs banale et se traduit par des attitudes particulières des Vers : "Torut éducateur un peu exercé, qui a le sentiment de la vigueur propre aux bons vers à l'époque de la montée, n'aura pas besoin de recourir au microscope pour s'assurer de l'état maladif de sa chambrée. la montée est lente, elle dure plusieurs jours, les Vers restant des heures entières immobiles sur les brindilles dont ils garnissent quelquefois le pied, comme s'ils hésitaient à aller plus avant. ") La graine de Sauve, exempte de corpuscules, souffrit à Alais, en 186 1 , de cette affection; les produits distribués en 1868, dans un grand nombre de petites éducations très soignées, donnèrent lieu partout à des échees complets uniquement dús à la flacherie. Désor. mais, il ne fut plus possible de douter que cette maladie aussi était héréditaire.

En raison de la banalité de l'affection, il sera d'ailleurs relativement facile de l'éviter. Des lots de Vers choisis, élevés dans des conditions particulières de propreté, donneront, au bout de quelques générations, des lots de Vers sains ; c'est une maladie bactérienne transmise surtout par contagion, exceptionnellement par les œufs. Des circonstances favorables à la pullulation du microbe, circonstances qui peuvent être extérieures aux Vers, nourriture mouillée, chaleur convenable, détermineront des épidémies funestes. PASTEUR rencontre ici, pour la première fois, les cireonstances qui déterminent les épidémies.

Sans doute, les corpuscules doivent aussi se multiplier 
dans des circonstances particulières, ralentissant la croissance normale des Vers et l'évolution régulière des organes. Mais ici les germes font, en quelque sorte, partie des Vers atteints, et lorsqu'ils ne s'y développent qu'en faible quan. tité, il n'y a ni mortalité, ni même déchet sensible dans la production des cocons. La maladie n'est pas une conséquence accessoire de la multiplication extrême dans un tube digestif extérieur à l'animal ; elle n'est grave que si le parasite, intimement adapté à son hôte, envahit tous les tissus; en un mot, le corpuscule de la pébrine est dangereux au même titre que la douve du foie, que la plupart des organismes qui vivent intimement associés à un hôte défini. Il y a parallélisme d'états entre la Rouille attaquant telle on telle Céréale, avec laquelle elle constitue un complexe défini, et la Pébrine dans le corps du Ver à soie.

Pasteur a reconnu la nature microbienne du parasite de la flacherie; sa forme en bâtomnets, la distribution en chapelets, sont caractéristiques de la multiplication des bactéries. Il a eu le sentiment, qui apparaît clairement dans ses conclusions, que le corpuscule était d'une toute antre nature. Les progrès accomplis an cours des cinquante dernières ammées, dans la comnaissance des parasites sporozoaires, vont nous montrer la différence essentielle entre les deux natures des parasites.

D'après PAS'TEUR lui-même, il y a lien de distinguer deux étapes dans la vie du corpuscule. L'élément brillant, d'aspect nacré, à contour ovale bien délimité, est le corpuscule âgé, incapable de se multiplier s'il a été exposé à l'air ou à la lumière; d'où l'innocuité des poussières des nagnaneries aérées et grossièrement désinfectées au point de vue de la propagation d'une année ì l'autre de la pébrine. Trais il y 
a un autre état des corpuscules où leur protoplasma n'a pas acquis la transparence ni la netteté des contours; à cette phase, ils se divisent rapidement, manifestent une grande activité et envahissent tous les tissus des chenilles et des chrysalides.

BALBIANT, à cette époque, faisait connaître un assez grand nombre de parasites des tissus profonds des Poissons. En 1887, LEUCKART réunit toutes ces formes de microorganismes à évolution compliquée sous le nom de sporozoaires et, en 1895, Balbiani et Henneguy provoquaient la publication d'un Mémoire de Théolan, intitnlé Recherches sur les Myxosporidies, parmi lesquelles figure la pébrine du Ver à soie, sous le nom de Gilugea bombycis. Les animaux de cette classe présentent à la fois, des formes libres et des formes fixées, dont nous allons suivre rapidement les métamorphoses d'après les travaux récents de StEMPELL (1909), qui đécrit sous le nom Nosema bombycis la cause de la pébrine.

Dans une cellule intestinale à plateau du Ver à soie, dont le noyau (fig. $24, N$ ) est volumineux, Stempeld découvre de petits éléments ovalaires ou pyriformes, libres, ou en voie de division, ou accolés par deux ou par quatre; c'est le stade méronte $m$, qui correspond à une phase de multiplication intracellulaire et de maturation. Elle aboutit à la spore jeune $s p . j$. expulsée ou non de la cellule au stade adulte ou de repos, auquel correspond l'organisation de la spore nacrée et brillante, décrite par PASTEUn sous nom de corpuseule âgé. Un très fort grossissement permet d'y découlvrir des détails remarquables : la spore (fig. 24 à droite) a la forme d'un œuf dont le gros bout porte une calotte distincte d'où part un filament replié sur lui-même et roulé en spirale; le contenu cellulaire granuleux remplit l'intervalle compris entre la coque et la capsule interne où se trouve 
le filament. Lorsque les circonstances deviennent favorables, et sans doute au cours de la germination de l'couf du Ver à soie, le filament se dévagine et entraîne la spore $s p$. $f$. dans ses ondulations ; bientôt le filament se détache, tandis qu'une petite portion de protoplasme nu se dégage, augmente légè. rement de volume, se meut par des mouvements amiboïdes et atteint la paroi de la cellule voisine, à travers de laquelle

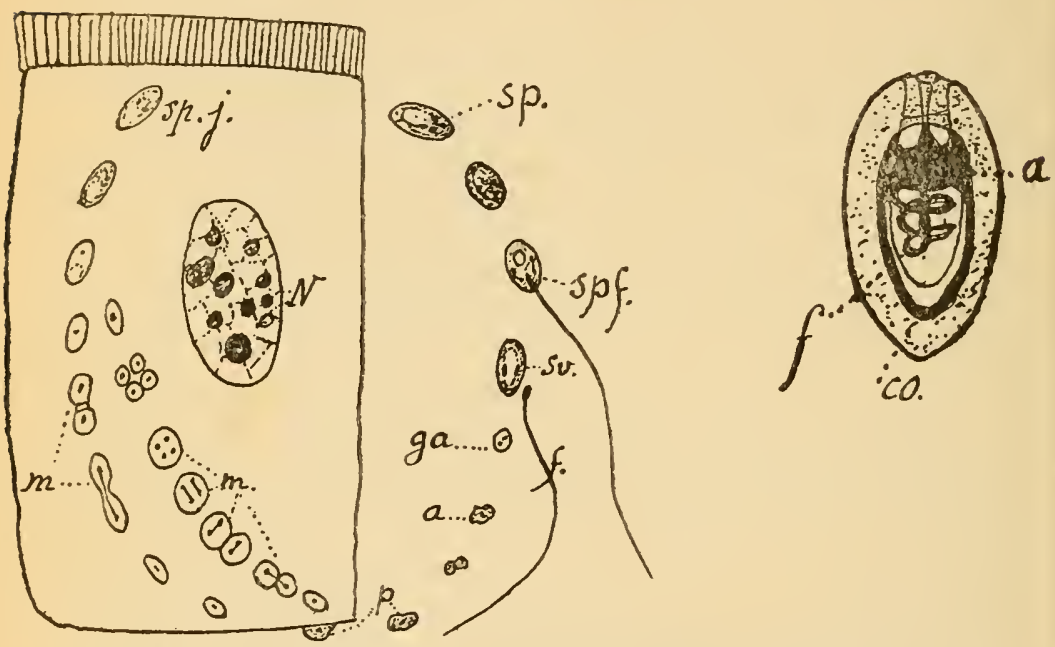

Fig. 24. - Cycle évolutif du Nosema bombycis (d'après StempelL).

il s'infiltre pour recommencer le cycle méronte. Il y a donc pénétration lente, qui souvent peut rester localisée.

PAsteur n'a pas connu les détails de cette organisation en raison de l'imperfection des microscopes qu'il avait à si disposition et aussi du fait que la technique des fixations histologiques était, à cette époque, très rudimentaire. Mais l'essentiel du mécanisme de la germination ne lui a pas échappé, comme le montre le dessin ci-contre, reproduit 
d'après son ouvrage (fig. 25); l'ouverture de la eoque du corpuscule et l'abandon d'un granule y sont parfaitement représentés.

Pour cette raison, je crois ne pas faire erreur en citant (1) PASTEUR comme le premier savant qui ait établi, d'une manière indiscutable, l'hérédité de complexes d'espèces vivantes; il faut lui accorder ce mérite d'avoir, par une incursion de quelques années dans le domaine des naturalistes, qui lui était à cette époque totalement étranger, mis en évidence la possibilité d'une altération durable des germes à travers la reproduction sexuée.

DARwin, en 1868, par conséquent à la date même où PASTEur établissait, par des épreuves expérimentales et d'une manière défini-
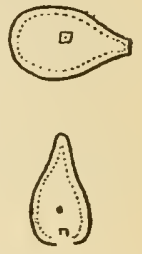

Fig. 25. - Corpuscule s'iuvrant (d'après Pas TEUR). tive, le caractère héréditaire du parasite caractéristique de la maladie de la pébrine, $D_{A R W I N}$, dis-je, présentait timidement l'hypothèse de la pangénèse, combattue de suite, et à laquelle HUGO DE VRIES substituait (1889) la pangénèse intracellulaire, origine de ses études sur la mutabilité des espèces.

" Les hypothèses, quoique incomplètes ou même erronnées, dit DARWIN, peuvent souvent rendre des services à la science. C'est à ce point de vue que je risque l'hypothèse de la pangénèse, qui implique que, dans l'organisme tout entier, chacun des atomes ou unités qui le composent se reproduit lui-même. D'où les ovules et les grains de pollen, la graine fécondée ou l'œuf, ainsi que les bourgeons consistent en, et comprennent, une multitude de germes émanant de chacun des atomes séparés de l'organisme. ") (1).

1. - Dans un ouvrage en préparation sur L'Hérédité acquise. 
Je n'ai pas l'intention de soutenir la validité de tous les arguments fournis par DARwiN en faveur de son hypothèse: la plupart sont erronés; mais je soupçonne que l'on a trop négligé, dans l'explication de nombreux phénomènes aberrants de l'hérédité, les circonstances accessoires qui ont pu introduire dans un organisme donné des particules qui persistent, à travers la reproduction sexuée, ou même qui, s'ils sont vivants, se fragmentent, affectent tous les ovules (champignons de la graine de l'Ivraie) et constituent des caractères permanents des espèces. 


\section{LES MODIFICATIONS DE L'INDIVIDU}

CHAPITRE VII

\section{PLASTICITÉ DES FERMENTS FIGURÉS ET LIGNÉES PURES}

En 1S67, Рh. van Tieghem publia un Mémoire sur la fermentation gallique, manifestement inspiré des travaux de Pasteur (1) ; même technique expérimentale, mêmes hypothèses sur le rôle des ferments, même contrôle de leurs conséquences logiques. Le fait nouveau est que le ferment est un organisme d'ordre supérieur. En réalité, deux Champignons très voisins, Pönicillium glaucum et Aspergillus niger, sont capables de provoquer la transformation du tannin en acide gallique.

Van TIEGHEm montre que le tannin ne se transforme pas à l'abri de l'air ; les dissolutions aqueuses privées d'air par le vide prolongé, saturées par un courant d'acide car-

1. - Recherches pour servir à l'histoire physiologique des Mucédinées. Fermentation gallique, Ann. Sc. Naturelles, Botanique, 5' série, t. VIII, p. 210. 
bonique ou de tout autre gaz inerte, ne se modifient pas au long d'un année, lorsqu'elles sont préparées et bouchées convenablement. L'oxygène est nécessaire à la transformation. Si l'on prend soin de faire bouillir les solutions dans les flacons à col ouvert et recourbé, suivant le procédé de Pasteur, la conservation est indéfinie, même en présence d'oxygène. Pour que le tannin se transforme, il faut, et il suffit, qu'un mycélium de Champignon (Mucédinée) se développe dans la solution :

“ Semons maintenant, dans un des ballons à col recourbé qui, laissés à eux-mêmes, ne s'altèrent jamais, quelques spores de l'une ou de l'autre de ces Mucédinées; empêchons toute végétation superficielle et nous verrons, en même temps que les spores développeront dans l'intérieur du liquide de beaux flocons de mycélium, le tannin subir une destruction progressive qui se trahira bientôt par la formation de cristaux de plus en plus nombreux d'acide gallique et qui, après quelques jours, sera complète. ")

L'air dissous apporte au tannin à la fois les spores et l'oxygène nécessaires à la fermentation ; dans le tannin pur et l'eau distillée, le développement du mycélium est nul ; qu'on y ajoute une petite quantité de nitrate d'ammoniaque et de cendres de bois, la destruction du tannin est aussi rapide que celle de l'extrait de noix de galle non purifié ; le Champignon a besoin de principes minéraux et azotés pour croître.

Un faible volume d'oxygène, par exemple 8 centimètres cubes, suffiront pour transformer, et au delà, 20 grammes de tannin ; le poids du mycélium n'atteindra pas le millième du poids du corps transformé. L'analyse montre que la plante emprunte au glucose, produit de dédoublement du tannin, le carbone nécessaire à la constitution de ses tissus 
et à sa respiration, qui doit être lente. C'est un type d'une fermentation par vie presque sans air analogue à celui de la fermentation alcoolique.

Le dédoublement du tannin en glucose et acide gallique ne s'opère intégralement que si la plante vit dans la profondeur de la dissolution. Dans ces conditions, son développement est lent et très faible. Mais, laisse-t-on la Mucédinée s'étaler à la surface du liquide, elle forme bientôt une membrane continue et ses filaments dressés se couvrent d'innombrables fructifications. Dans ces conditions, le Champignon brûle tout le glucose d'abord, puis l'acide gallique presque entièrement; son poids augmente jusqu'à atteindre le quart du poids du tannin détruit. "Ces combustions rapides et totales de matières organiques par la végétation superficielle des végétaux microscopiques et le rôle important qu'elles jouent dans l'économie de la nature ont été étudiés pour la première fois par MI. PASTEUR; nous en voyons ici, dit V VN Tieghem, un nouvel et curieux exemple. "

Curieux, en effet, car si le ferment a besoin de traces d'oxygène pour vivre, il emprunte au tannin non pas l'oxygène mais le sucre, seul aliment utilisable par le Champignon vivant en profondeur. A la surface, le Champignon brûle l'acide gallique qu'il laisse intact en vie anaérobie.

Le Penicillium glaucum, utilisé par Van Tieghen, est précisément celui qui décompose le paratartrate acide d'ammoniaque en ses tartrates droits et gauches, pour détruire le sel droit et isoler le sel gauche. "Dans des expériences encore inédites, dit Van Tieghem en note, MI. Pasteur a reconnu : $1^{\circ}$ que le Penicillium glaucum peut détruire à son tour le tartrate gauche; $2^{\circ}$ que les tartrates gauches de chaux et d'ammoniaque pouvent, eux aussi, fermenter, 
quoique beaucoup plus difficilement que les sels droits correspondants. " On sait que c'est une espèce très banale, la la vulgaire moisissure à reflets bleus, qui envahit le pain, les confitures, le fromage.

L'autre 'Mucédinée qui provoque la fermentation gallique, nommée Aspergillus niger, est presque aussi commune; elle se développe sur le pain movillé, sur les tranches de eitron et d'orange, sur les feuilles mortes, sur des milieux minéraux; RAULIN (1870) l'a prise comme type pour l'étude systématique de l'influence des éléments constitutifs des milieux de culture artificiels formés de substances minérales, et c'est un des êtres dont les conditions d'alimentation sont les mieux connues.

L'une et l'autre Mucédinées sont aptes à vivre dans des conditions variées; elles présentent des modifications d'aspect troublantes que nous allons maintenant examiner.

En profondeur, les filaments de l'Aspergillus niger forment des flocons lâches, diffus, sans reflets irisés (ce qui les distingue à première vue des filaments du Penicillium glaucum), qui s'étalent en masse irrégulière nageante s'accroissant régulièrement sur toutes ses faces. A la surface des solutions nutritives, les flocons sont beaucoup plus denses ; ils s'étalent en disques qui eroissent par zones concentriques si régulières qu'on peut suivre les variations de température à la propagation inégale des ondes. Bientôt les disques se rencontrent, et l'ensemble forme une membrane superficielle continue. A cette phase, la forme fructifère apparaît, alors qu'elle ne se produit jamais en profondeur. Perpendieulairement à la surface des disques, des filaments simples non cloisonnés, cylindriques, dont les membranes s'épaississent fortement avec l'âge, atteignent jusqu'à un millimètre de hauteur ; leur extrémité se renfle 
en un capitule sphérique d'abord lisse, puis verruqueux, chaque verrue devenant les pédicelles rayonnants extrême. ment serrés qui se couvrent de spores, d'abord jaunes citron, puis brun chocolat ou noires. En résumé, cette plante offre deux formes, l'une stérile, qui vit en profondeur, ne fructifie pas et ne décompose pas l'acide gallique ; l'autre fertile, qui vit à la surface de tous les milieux nutritifs, les décompose en eau et acide carbonique et donne naissance à des organes de reproduction caractéristiques. Le changement dans les conditions de vie permet d'obtenir, à partir d'un fragment de l'une ou l'autre forme, les deux états, qui sont un bon exemple de l'adaptation double étudiée précédemment (page 30) chez les végétaux supérieurs.

L'Aspergillus résiste mieux que le Penicillium à la vie sans air ; à la limite, ses filaments présentent des déformations curieuses dont il faut dire quelques mots. Au contact de l'air, le filament est cylindrique, très régulier, d'un diamètre de 3 à $4 \mu$ (millièmes de millimètres), les cellules étant environ vingt fois plus longues que larges; lorsqu'il est maintenu en profondeur et que l'oxygène a totalement disparu, les cloisons transversales deviennent plus nombreuses, les filaments présentent en même temps des renflements dont le diamètre peut atteindre $20 \mu$, e'està-dire cinq fois le diamètre du filament normal. Dans ces conditions, les filaments apparaissent comme des chaînes de cellules de levure qui ne se sépareraient pas encore complètement les unes des autres.

Avec le Nucor racemosus, Champignon voisin des Aspergillus, qui forme ses spores à l'intérieur de la boule qui termine le filament dressé, la vie anaérobie entraîne l'apparition de tous les caractères des levures proprement dites, 
A. Fitz (1875) a fait une étude approfondie de ce végétal et des fermentations qu'il provoque.

On obtient, presque à coup sûr, Mucor raccmosus tỵpe en exposant à l'étuve, dans une assiette, un peu de erottin de cheval ; on le recommait aux touffes blanches argentées qu'il forme, et il est facile de le récolter pur et de le eultiver à l'abri de l'air sur des milieux acides, tranches de poire ou de citron. Il vit très bien dans mne solution de sucre de lait, mais ne la fait fermenter que si le sucre a été préalablement interverti. Ensemencé pur, n'ayant pas à lutter contre les levures ordinaires, il se développe parfaitement sur les moûts de bière stérilisés par la chaleur.

Vivant comme une moisissure à la surface des corps humides acidulés, là où l'air pénètre facilement, Mucor race. mosus donne une abondance de tubes fructifères aériens. Plongeons-le dans le fond du liquide; ses spores, s'il y en a d'entrainées, grossissent et atteignent des dimensions linéaires doubles avant de germer; parfois même, elles se gonflent davantage et ne se cloisonnent qu'après avoir émis des bourgeons qui rappellent ceux des levures ; les filaments mycéliens qui en sortent forment des articles courts, au plus quatre à cinq fois plus longs que larges, et leurs ramifications, qui se détachent souvent par groupes de deux ou trois, fournissent, à côté des cellules longues (fig. 26), des chapelets de cellules rondes de tailles et de formes très variables. Au fur et à mesure que la culture vieillit, tou. jours à l'abri de l'air, les tubes sont moins fréquents et les cellules rondes augmentent en nombre et en variété. "La ressemblance avec des cellules de levure, dit Ductaux (1), est alors tellement frappante, que le botaniste BaIL, en 1857,

1. - Traité de Microbiologie, t. III, p. 18. 
avait cru à la transformation des mucors en levures. La culture pure en ballons comme l'a faite M. PASTEUR prouve, comme REEss l'avait du reste déjà vu, que cette transformation n'a pas lieu, et que la fermentation qui se produit est le fait des spores mycélieunes. " L'analogie avec les modifications que j'ai signalées chez Polygonum amphibium (p. 30) m'en paraît renforcée. Ici encore, les bourgeons évolués se détruisent avec leur forme première ; ce sont les jeunes bourgeons soumis à l'action du milieu nouveau, avant d'avoir fourni les cloisommements typiques dus à l'éveil de

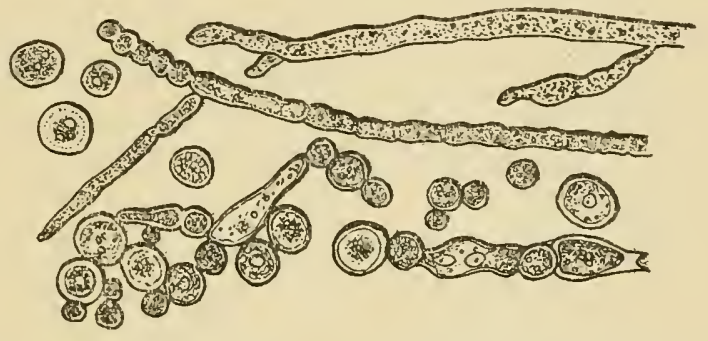

Fig. 26. - Variations de forme du Mucor racemosus cultivé en profondeur du moût (d'après Duchaux).

la eroissance, qui donnent d'emblée les tissus et les arrangements symétriques caractéristiques de leur espèce.

Avec l'âge et maintenues à l'abri de l'air pendant longtemps, les cellules rondes ou ovoïdes du Mucor racenısus se désorganisent peu à peu ; lcur protoplasma devient granuleux et se rassemble au centre ; après cett: phase, ii devient presque impossible de les régénérer. On assiste à des phénomènes d'autodigestion qui précèdent la mort.

Il est remarquable qu'un mycélium défini, donnant naissance dans l'air à des formes parfaitement et facilement déterminables, se métamorphose en granulations cellulaires 
du type des levures, ou même en masses protoplasmiques plus condensées encore, avant d'être totalement privées de leur pouvoir de rajeunissement. N'y a-t-il pas dans ces phénomènes, dus à la vie anaérobie, des indications précieuses pour fournir à l'hypothèse d'ERIKsson sur le mycoplasma des Rouilles quelques arouments qu'il serait bon de préciser?

*ै* *

Parallèlement à ses études sur la maladie du Ver à soie, PASTEur poursuivit ses recherches sur les fermentations et plus spécialement sur les procédés de fabrication des deux boissons nationales, le Vin et la Bière. Ses travaux ont eu un légitime succès et sont le point de départ de ses découvertes sur le polymorphisme des agents de la fermentation et de la putréfaction.

Le titre des Etudes sur le Vin (1866) est complété par le résumé des préoccupations du savant qui veut faire œuvre utile : ses maladies, eauses qui les provoquent; procédés nouveaux pour le conserver et pour le vieillir. Les conclusions de PASTEUR sont formelles ; il faut autant que possible réaliser les conditions d'une bonne fermentation normale, e'est-à-dire favoriser le développement du ferment propre et supprimer les ferments accessoires. Le procédé de Pasteurisation, ou chauffage à cinquante degrés des moûts, empêche les maladies, ascescence, tourne, graisse, amertume, toutes dues à des microorganismes particuliers. Nous passons rapidement sur ces points bien connus.

Il faut surtout insister ici sur le rôle essentiel de l'oxygène dissous et sur les circonstances de fabrication qui mettent l'oxygène en présence du vin au cours du foulage, des soutirages, de la mise en bouteilles. "Lorsque le moût est 
exposé au contact de l'air en grande surface pendant plusieurs heures, ou agité avec de l'air..., la fermentation du moût est incomparablement plus active que celle du même moût non aéré et la différence varie avec l'intensité de l'aération... Il ne faudrait pas croire que ces différences tiennent au poids de gaz oxygène que l'aération peut fixer sur les principes du mon̂t. C'est à une constitution propre de la levure qui prend naissance qu'il faut les attribuer ")(1). PASTEur signale lui-même que, dès 1861 , dans sa communication à l'Académie des Sciences intitulée Expériences et vues nouvelles sur la nature des fermentations, il a déjà insisté sur le fait de la rapidité de multiplication du ferment du moût de raisin exposé au contact de l'air et qu'il a constaté que le ferment, considéré en lui-même, abstraction faite du poids qui s'est formé au cours de l'opération, est un ferment des plus énergiques lorsqu'on le fait agir ensuite sur du sucre à l'abri de l'air. D'ailleurs cette activité exaltée ne dure pas ; elle s'épuise très vite.

"Il résulte de ces faits que l'on peut modifier considérablement une variété déterminée d'un ferment alcoolique, changer ses propriétés physiologiques par le seul fait d'un changement dans les conditions de son développement. Les différences sont telles que l'on pourrait croire à des espèces distinctes. Il faut done être très sobre de déductions sur la nature spécifique des ferments, alors même que l'on constate des modifications importantes dans leur manière d'agir » (1).

En fait, à cette date et même au cours des dix années suivantes, les Annales botaniques et chimiques sont encom-

1. - Etudes sur le vin, pp. 194, 197. 
brées de Mrémoires et de Notes qui sont visiblement inspirées des idées que le transformisme de Darwin, dans tout son éclat, suggérait à ceux qui n'avaient pas acquis une technique de l'étude des microorganismes suffisamment précise. BÉCHAMP, en 1871, soutint que les bactéries du vinaigre sont capables de se transformer en levures; Trécul, botaniste renommé, affirma en 1872 avoir réalisé la métamorphose des spores du Penicillum en véritables levures alcooliques, et, en 1875 , Сн. RoBin prétendit que plusieurs levures (Torula cerevisiae, Mycoderma cerevisiae) ne sont, avec le Penicillum glaucum qu'une seule et même espèce. Les exemples de métamorphose des ferments du tannin, étudiés par VAN TIEGHEMr, donnaient quelque poids à ces affirmations.

La sagacité de Pasteur, mise en éveil, lui fit découvrir que plusieurs espèces indépendantes de levures apparaissent suceessivement dans le moût de raisin au cours de la fabrication du vin. C'est d'abord une petite levure, qu'on isole facilement en filtrant grossièrement le moût; il passe un Saccharomyces apiculatus qui, dans les conditions ordinaires, est bientôt étouffé par l'extension d'une forme beancoup plus vigoureuse, Saccharomyces pastorianus ; à la fin de la fermentation, on voit généralement apparaître S. ellipsoïdeus. Les progrès de la détermination et de la classification de ces formes réduites de champignons, surtout réalisés an Laboratoire danois de Carlsberg, sons la direction de IIANSEn (1881-1890), ont provoqué la distribution de ces diverses levures dans des sous-genres différents.

La première est devenue, après la découverte de la forination des ascospores par Lindner (1903), le type du nouveau genre Hansenia. $H$. apiculata (fig. 27, $I$ ), abondante sur les fruits sucrés et mûrs, est caractérisée par la présence 
de deux petites pointes on mamelons anx extrémités de Ia cellule, dont le contour ovale prend la forme d'um citron; mais ces mêmes cellules peuvent rester ovales, perdre et reprendre une de leurs pointes, se déformer en demi-lune, s'allonger en bactéries; les formes apiculées sont des formes jeunes de l'être en voie de pullulation, les formes ovales celles d'êtres âgés. Alors que HaNSEN n'a jamais pu en obtenir d'asques, Lindner a réussi à observer une seule ascospore dans les cellules apiculées isolées des fleurs du Robinia

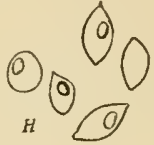
pseud'acacia. En admettant qu'on puisse identifier' les deux types, il faut croire que la sporulation est pour certaines races particulièrement difficile et même assez rare dans la nature.

Le Saccharomyces Pastorianus de ReEss est décomposé par HANsen (1883-1902) en types I, II, III, ì formes différentes, avec asques renfermant de un à dix ascospores, les nombres les plus fréquents étant

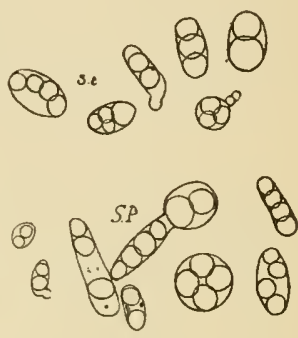

Fig. 27. - Diverses sortes de levures dans la fermentation normale du vin; $H$, Hansenia apiculata;Se, Saccharomyces ellipsoîdeus: $S P$, Saccharomyces Pastorianus représentées dans la phase de sporulation (d'après HANSBN et LiNDNER). compris entre 3 et 5 ; la taille des ascopores, comme celle des asques, varie dans des proportions étonnantes, oscillant entre 1,5 et 5 millièmes de millimètre. En fait, c'est un groupe très instable (fig. $27, S P$ ).

Le Saccharomyces ellipsoïdeus de ReEss est une levure basse à cellules presque toujours elliptiques, rarement allongées, avec asques donnant de 1 à $t$ ascospores de taille variant entre 2 et 5 millièmes de millimètre. Dans les cul- 
tures de moût de bière additionné de gélatine, la levure ensemencée en strie se dispose en réseau d'articles tout à fait caractéristiques (fig. $27, S e$ ).

PASTEur prépare ces savantes distinctions par la mise au point de la technique des cultures pures. Pendant quelques années, il subit cependant l'influence des idées courantes et il n'est pas sans intérêt de savoir pourquoi il crut d'abord à la métamorphose d'une espèce en une autre, et comment il réussit à se dégager d'une idée préconçue, qu'il prouva lui-même être erronée. (Etudes sur la bière, 1873).

Lorsque des cellules levures se trouvent dans un milieu nutritif convenable, elles bourgeonnent en reproduisant des cellules identiques aux cellules mères; dans un milieu inca. pable de les nourrir, tel que l'eau sucrée pure, elles bourgeonnent difficilement, brunissent et se déforment. Semons une levure ainsi épuisée dans un liquide nutritif complet, tel que le moût de bière ; elle se rajeunit, donne des cellules nouvelles différentes de la forme primitive qui, dans le même mon̂t, se stabilise. "Le Saccharomyces pastorianus nons offre en conséquence un trait d'union entre le genre levure et certains genres de moisissures vulgaires, notamment le genre que DE BARY nomme dematium dont l'habitat ordinaire est la surface des feuilles et des bois morts et qui est surtout d'une abondance extraordinaire sur les bois de la vigne à la fin de l'été, au moment de l'époque des vendanges. Tout porte à croire qu'à cette époque de l'année un ou plusieurs de ces dematium fournissent des cellules de levure ou que même les dematium aérobies ordinaires émettent à un certain moment de leur végétation, outre des cel- 
Iules et des torula aérobies, d'antres cellules et torula anaérobies, c'est-à-dire des levures alcooliques.

"Nous arrivons ainsi à la confirmation d'un soupçon qu'ont eu la plupart des auteurs qui ont beaucoup observé la levure, c'est qu'elle devrait être un organe détaché d'un végétal plus complexe. Ainsi le Saccharomyces en question forme des chaînes de tubes, de fuseaux, d'articles qui rappellent extrêmement les chaînes de tubes, de cellules-boules ou conidies du Mucor racemosus submergé, de telle sorte qu'on pourrait croire que notre dematium-levure est luimême, dans ses spores germes, un organe détaché d'un végétal plus complexe, comme la levure en boules appartient à la moisissure plus complexe, le Mucor racemosus. "

On ignore d'ailleurs l'origine et l'histoire physiologique de la levure employée de nos jours en brasserie, et PASTEUR suggère que ce ne sont pas les seules qu'on pourrait obtenir. Il tente des essais en ce sens et en donne le principe : " Une levure est une réunion de cellules qui ne sauraient être individuellement identiques. Chacune de ces cellules a des propriétés d'espèce ou de race, qu'elle partage avec les cellules voisines et, en outre, des caractères propres qui la distinguent et qu'elle est susceptible de transmettre dans des générations successives. Si done on parvenait à isoler' dans une levure déterminée les diverses cellules qui la conposent et qu'on pût cultiver à part chacune d'entre elles, nn obtiendrait un nombre égal de levures qui vraisemblablement seraient distinctes les unes des autres, parce qu'elles participeraient chacune des propriétés individuelles de leurs cellules origines. "Les levures actuelles sont done des sélections de l'industrie ; on peut imaginer qu'on en obtiendrait facilement un grand nombre d'autres. 
Souvent Pasteur met en lumière sa pensée ell empruntant des exemples à nue catégorie d'objets mieux connus ; ceux qu'il choisit pour faire comprendre l'origine des levures industrielles m'ont beaucoup frappé dès 1902 , époque à laquelle je me suis livré à des études de sélection des Céréales. Jamais, dans ses propres recherches, qui ont fait apparaître tant de formes levures à partir d'organismes plus différenciés, il n'a obtenu les deux levures de la bière qu'on se procure si facilement dans le commerce. "Je suis porté à croire, dit-il, que nous avons ici un exemple nouveau de ces modifications de plantes ou de races d'animaux devenus héréditaires par une domestication prolongée. On ne connaît pas le blé à l'état sauvage ; on ne sait quelle a été sa première graine. On ne connaît pas non plus le ver à soie à l'état sauvage; on ignore la race qui en a produit le premier œuf. )

Développous la comparaison, qui nous fera apparaître plus clairement cette pensée si profonde, mais pas toujours percue dans sa netteté et dans ses conséquences, parce qu'elle porte sur des êtres microscopiques dont les particularités sont délicates à saisir et à traduire.

En 1901, Giard m'engageait résolument dans un travai] original, difficile à conduire parce qu'il exigeait la culture en grand, dans des champs rispersés, de lignées tératologiques de Maïs. Il avait compris à la fois la portée des épreuves et la nécessité d'une longue suite de contrôles coûteux. Un de ses élèves et collaborateurs, M. Mesnin, chef de service à l'Institut Pasteur', apprit par lui l'orientation de mes travaux et, sachant qu'un syndicat professionnel de brasscurs voulait faire sélectionner les Orges françaises utilisées pour le malt, me signala au Président de ce syı- 
dicat, M. AD. Kreiss, comme susceptible de l'aider. Au cours des premières conversations sur le sujet, alors que II. Kreiss me proposait de former un chimiste anx méthodes de sélection des botanistes, je compris l'intérêt théorique et pratique des recherches à faire et je pris la résolution d'étudier moi-même le problème posé.

La Société d'Encouragement de la Culture des Orges de Brasscrie, section du Syndicat des Brasseurs français, qui s'était chargée des études, avait introduit en France diverses sortes réputées de Bohême, de Suède, donnant des commodités de fabrication et de hauts rendements en bière. Il s'agissait de les suivre et d'en contrôler la pureté. Tout de suite, j'eus l'intuition qu'il s'agissait de faire des cultures pures selon les principes déconverts par PASTEUR et que m'avait enseignés Duclaux; les dirigeants de la Société, IINI. A. Kreiss et P. Petit, tous deux pastoriens, me confirmèrent dans cette opinion. En fait, il s'agissait de trier dans les Orges françaises autant de types qu'il était possible d'en rencontrer, de même que PASTEur avait suggéré qu'on pouvait retirer des levures communes de la brasserie des lots plus homogènes, purs, parce que dérivés d'une seule cellule multipliée dans des milieux de cultures définis. La suggestion de PASTEur était réalisée en fait depuis une quinzaine d'années au Laboratoire de Carlsberg, à Copenhague, fondé par le célèbre brasseur danois $J_{A C O B S E N}$ et dirigé par HANSEN, le systématicien des levures.

En 1902, les idées de tout biologiste s'occnpant de sélection étaient dominées d'une part par les travaux de M. Hugo de VRIES sur la variation brusque et héréditaire, d'autre part par la fameuse notion des lignes pures dont M. Johannsen venait de faire connaître la fixité et les caractères par une étude approfondie d'une vieille race de Hari- 
cots cultivée au Danemark (1). Je rendis visite, en février 1902, à M. Johannsen, qui, au cours de la démonstration des recherches faites dans son Laboratoire, me proposa une visite à l'Institut des levures pures de Carlsberg. Depuis cette visite, ma convietion s'est affirmée. L'idée qui a guidé JoHANNSEn dans ses études, qui a servi de point de départ aux réalisations techniques de N. HJalmar NiLSSON à Svalöf (Suède), est une application aux Céréales cultivées du principe de PASTEUR : la séparation, à partir de l'ensemencement d'une cellule unique, des éléments constituant les variétés industrielles et agricoles, de quelque famille qu'elles soient, Levures ou Bactéries, ou Graminées. Voici ee que je reconnus dans mes cultures pures des Orges françaises destinées à la brasserie, faites à Bourg-la-Reine et à Villacoublay (1903-1914).

Dans un lot commereial d'Orge examiné au Laboratoire, on réussit à distinguer par les caractères des semences, des petites espèces ( $p .8$ ), et par les caractères des épis, des sortes (2). Mais ce $\mathbf{n}$ 'est pas le dernier terme de la séparation en types distinets; semons dans une plate-bande bien dressée, en lignes parallèles, les grains de dix, de vingt épis d'rune même sorte, l'orge Chevalier français, par exemple ; on constatera à la levée, à l'étalement des premières feuilles en rosette, à la montée des chaumes, surtout au dégagement de l'épi de la gaine du premier chaume, des particularités de port, d'étalement, de virage des teintes, etc., qui montrent que les graines d'un même épi donnent des plantes beaucoup plus voisines les unes des autres que celles qui dérivent des divers épis de la même sorte ; on ne peut tra-

1. - W. Johannsen : Uber Erblichkeit in Populationen und in reinen Linien, Jena, 1903.

2. - L. Blaringhem : Les Crus d'Orges de Brasserie, Paris, 1910. 
duire ces divergences par des caractères visibles sur les grains et sur les épis; il faut avoir recours à des cultures comparées côte à côte, dans les conditions les plus identiques, pour les mettre en valeur.

Certaines lignes, e'est-à-dire certains épis dérivant donc d'une même cellule œuf de la culture précédente, offrent une homogénéité complète; l'observation la plus méticuleuse ne permet pas de distinguer des différences entre les plantes. Conservons-les précieủsement et semons-les à part dans les mêmes conditions ; la plupart d'entre elles accuseront pour toute leur descendance cette uniformité d'aspect, à toutes les phases de la croissance, qui caractérise les cultures des graines d'un même épi, et cependant nous possédons maintenant plusieurs centaines de plantes dérivées. Continuons la culture en ayant soin de ne jamais y introduire aucune plante, ancun épi, aucun grain d'une autre origine, d'une autre ligne. Au bout de cinq années, il y aura assez de semence poill couvrir un hectare, an bout de sept années, pour couvrir cent hectares. Si le point de départ a été bien choisi, l'homogénéité de la famille cultivée sur cent hectares est aussi complète que celle de la ligne comprenant vingt plantes au point de départ. C'est la définition même d'une lignée pédigrée (généalogique) complétée par le contrôle qui en fait une lignée pure :

"La lignée pure est un lot de plantes issu d'une plante unique dont les descendants et les fragments de descendants, de quelque génération que ce soit, n'offrent pas entre eux de différences plus grandes que celles qu'on peut trouver entre les fragments d'un seul individu multiplié à l'infini (1). ") Autrement dit, la reproduction sexuée n'in-

1. - L. Blaringhem : Les problèmes de l'hérédité expérimentale, 1919 , p. 40. 
troduit pas, par sa répétition, des modifications d'équilibre entre les différents produits du lot; la plante, l'organisme supérieur, se comporte comme les levures bourgeonnant dans un liquide qu'elles font fermenter.

Bien que lignées pures, les dérivés de certaines suites généalogiques présentent entre eux des déviations notables. II. Johannsen l'a constaté pour les Haricots, M. Nilsson pour les Avoines, moi-même pour les Orges ; il paraît impossible dans un temps limité, c'est-à-dire en douze à quinze générations, de limiter l'étendue de ces variations ; l'instabilité fait partie inhérente du patrimoine héréditaire de ces plantes autofécondées. Certaines, tels que les dérivés des Orges à deux rangs à épis dressés, doivent cette instabilité à une origine hybride récente, ou du moins des hybridations convenables fournissent des lignées héréditaires qui offrent les mêmes particularités ; d'autres trahissent leur origine hybride après des cultures répétées sous certains climats, en certains terrains, qui ne conviennent pas à l'équilibre de leurs caractères; d'autres, enfin, sont instables pour des raisons inconnues. La régularité des progénitures n'est en tous cas assurée que si à la pureté d'origine et à une tendance propre s'ajoutent les effets d'un régime, d'un mode de vie, aussi uniforme que possible. Par ce caractère encore, les lignées pures de Céréales strictement autofécondées se rapprochent des lignées pures de microorganismes dérivées, dans les cultures en ballons de PASTEUR, de la multiplication indéfinie d'me cellule unique, qu'on empêche de vieillir par le renouvellement aussi fréquent que possible du milieu nutritif. 


\section{***}

La méthode des cultures pures de levures et de bactéries, actuellement adoptée partout, offre toutes les sécurités théoriques, tout en étant d'un emploi relativement facile. Elle n'a pourtant pas été imaginée de suite et nous allons voir comment PASTEUr et ses émules l'ont peu à peu amenée à la perfection.

PASTEUR utilise l'observation rappelée plus haut, que les levures, bien qu'anaérobies facultatives, se multiplient rapidement dans les milieux très aérés; les ballons qu'il utilisera seront donc à surface large ; ils porteront deux trubuIures, l'une supérieure, coudée, allongée, recourbée en $U$ à son extrémité (fig. 28 A), l'autre latérale et courte; le bec de la partie recourbée et amincie est bouché par de la bourre d'amiante $a$, qui filtre l'air, retient les poussières, tout en permettant l'aération suffisante ; l'autre est obturé par un court tube de caoutchouc $c$, fermé lui-
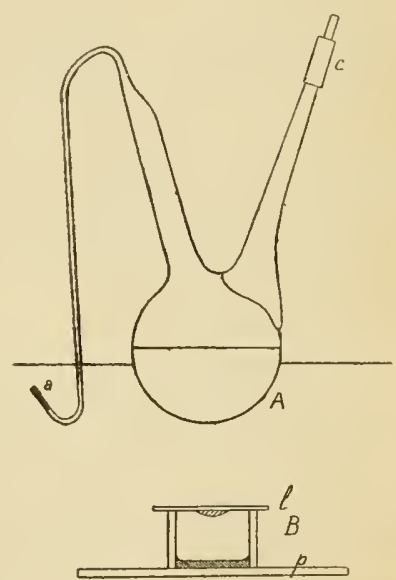

Fig. 28. - A, Flacon à deux tubulures imaginé par PASTBUR pour la séparation des lignées pures de ferments (d'après Ducraux); $B$, chambre humide de VAN TIEGHEM. même par un fragment de diamètre et de longueur convenable d'agitateur en verre plein.

Un des liquides les plus favorables au développement de la levure de bière est composé d'un extrait de moût de bière 
filtré et additionné de sucre pur cristallisé. PASTEUR employa souvent aussi un milieu purement minéral, composé de vingt grammes de sucre candi, un gramme de carbonate d'ammoniaque, un gramme de cendres de levures, le tout dilué dans un litre d'eau distillée.

La stérilisation s'obtient en faisant bouillir le ballon rempli à moitié ou en totalité durant au moins un quart d'heure ; on enlève la fermeture du tube latéral ; la vapeur s'échappe par son orifice largement ouvert, qu'on referme ensuite avec le caoutchouc muni de son agitateur préalablement stérilisé ; la vapeur s'échappe alors par le tube coudé et l'ébullition est maintenue pendant dix minutes ; on applique enfin sur l'orifice du tube coudé le bouchon d'amiante bien flambé. PASTEUr ensemence enfin avec des instraments flambés d'aussi faibles portions possibles d'une bonne levare active, qu'il introduit avec dextérité dans le ballon par ]a tubulure latérale.

D'ordinaire, la levure se développe très rapidement, élimine les bactéries (surtont en milieu légèrement acide) et les moisissures concurrentes. La répétition de ces opérations conduit plus ou moins rapidement à la préparation de cultures qui, par la destruction et l'étouffement des germes accessoires, dérive d'un petit nombre de cellules sœurs.

Pasteur ne fut pas satisfait de ce procédé, qui ne conduit que lentement et sans sécurité absolne à la préparation de levures pures. Imitant les jardiniers, qui mélangent préalablement de sable leurs lots de semences fines afin de les répartir uniformément et séparées les unes des autres, il imagina de broyer en pouảre aussi ténue que possible une petite portion de levure desséchée à trente degrés avec du plâtre ; il laissait tomber de très haut le mélange pour faire um nuage léger, et il ouvrait dans ce nuage un instant les ballons rem- 
plis de liquide nutritif où il avait fait préalablement un vide partiel. Quelques parties du nuage de plâtre et de levure pénétraient jusqu'au liquide et l'ensemençaient. En fait, il n'arriva pas encore à la perfection, et il fallut de nombreux et longs tâtonnements avant que HANSEN (18s11886) aboutisse à une méthode de séparation pratique, exacte et rigoureuse.

Une chambre humide (fig. 28 B) est composée d'une plaque de verre $p$ avec anneau de verre collé au baume de canada ; une lamelle $l$ mince de verre est posée sur le haut de l'anneau; une goutte d'eau mise dans la cuve y maintient l'atmosphère saturée. L'ensemble est stérilisé. On place au dedans de la chambre et au centre de la lamelle, avec des pipettes flambées, une petite goutte d'un liquide très dilué de levure ; la goutte s'étale et permet de dénombrer les cellules en voie de croissance sous le microscope. HANSEN emploie des couvre-objets à quadrillages numérotés et des milieux nutritifs à base de gélatine, qui se solidifient juste assez pour enfermer les cellules sans en gêner la multiplication au cours de l'observation au microscope. Il note les carrés où une seule cellule est visible, et il suit son développement jusqu'à la formation d'une petite colonie; la prise faite avec des instruments stériles dans le carré correspondant est an prélèvement de levures dérivant toutes d'une même cellule. Avec les précautions convenables, très simples maintenant, on peut indéfiniment conserver la culture pure par des ensemencements périodiques en tubes de culture renfermant des aliments convenables stérilisés.

Le même procédé permet l'étude exacte du pouvoir de multiplication des levures dans des milieux nutritifs et des conditions physiques déterminées. Il a permis de préciser 
les circonstances qui déterminent la sporulation. On sait maintenant que les Saccharomyces donnent naissance à de vraies asques, analogues à celles que donnent les Truffes, renfermant des nombres variables d'ascospores, nombres qui, nous l'avons vu, servent à la détermination des races. On a pu suivre l'évolution uucléaire et noter, avant la formation des asques, des copulations de noyaux qui rappellent les phénomènes de la sexualité des végétanx supérieurs. La
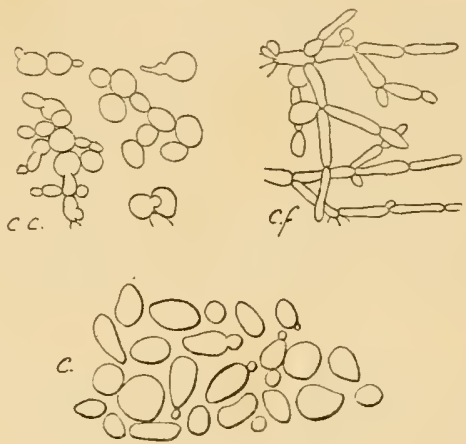

Fig. 29. - Variations du Saccharomyces Carlsbergensis; c, culture à la température de 25 deyrés et jeune; $c f$, culture à température très basse et âgée de plusieurs mois ; $c c$, culture à température très élevée et âgée de plusieurs mois (d'après HANSEN).

généralité du processus de la sexualité chez les êtres vivants est une des conquêtes du début du siècle et peut nous aider à pénétrer plus intimement dans ces phénomènes de dissymétrie moléculaire que PASTEur affirme exister dans tous les œufs. Nous y reviendrons ailleurs.

Hansen (1895-1898) réussit, avec sa technique irréprochable, à établir les circonstances qui permettent d'obtenir d'une seule cellule de Saccharomyces Carlsbergensis des formes d'aspects très différents (fig. 29) ; en particulier, il fit 
agir les températures basses et hautes à l'époque où STANDFUsS appliquait le même procédé pour obtenir les variations saisonnières des Papillons. A la température de vingt-sept degrés, les cellules se multiplient activement dans le moût de bière et donnent uniquement des cellules ovoïdes, rarement presque rondes (fig. $29 \mathrm{c}$ ) ; à cinq degrés centigrades, les mêmes cellules fournissent surtout des cellules allongées, mêlées à quelques rares cellules ovales ; à trente-trois degrés, le bourgeonnement est encore marqué, mais les articles des chaînes sont très courts avec des étranglements prononcés. Dans les deux cas, il y a des cellules géantes ; l'agencement en mycélium (fig. $29 c f$ ) est earactéristiqne des cultures à températures basses, l'agencement en rosettes (fig. $29 c c$ ), des températures hautes. Il faut souvent plusieurs mois pon $\mathrm{r}$ que l'organisation de ces états devienne caractéristique, la levure, dans les conditions extrêmes de température $\left(U^{\circ}\right.$ à $9^{\circ}$ et $30^{\circ}$ à $30^{\circ} 5$ ), ayant un développement très ralenti. Or, même après des années de vie dans ces conditions défavorables, les nouveaux caractères ne paraissent pas stables. Des prélèvements, placés dans les conditions favorables à une rapide multiplication, fournissent bientôt la forme ordinaire à cellules ovales, douée comme ses ancêtres d'une grande activité dans les conditions de préparation des bières à fermentation basse.

D'autres variations accidentelles de la même levure, lui donnant l'aspect des Saccharomyces cerevisiae, ou encore des S. Pastorianus, ont pu être conservées dans plusieurs séries de cultures durant plusieurs mois, mais en définitive n'ont pu être fixées. Cette levure présente donce, à la fois, les variations que nous avons étudiées sous les noms d'adaptations doubles et aussi l'instabilité que j'ai observée dans les Orges de Bourbourg, Cistercienne et autres ; on conçoit combien 
ces phénomènes compliquent l'étude des changements spécifiques.

Hansen a obtenu, dans certaines circonstances et en isolant un très grand nombre de cellules du Saccharomyces Ludwigii, trois sortes, que certains auteurs désignent comme sous-espèces parce qu'elles diffèrent par leur faculté de sporuler. L'espèce type fut trouvée par Ludwig dans les sécrétions muqueuses du Chêne, et Hansen (1889) l'étudia en notant ses particularités de division qui sont, à beaucoup d'égards, analogues à celles des Champignons filamenteux. Placée dans une solution aqueuse de saccharose on dans l'eau de levure, elle forme rapidement des asques fuli renferment quatre ascospores rondes. Par un choix métliodique des cultures à partir d'une même cellule, Hansen a obtenu trois races différentes, l'une très riche en asques, la seconde pauvre en asques, la troisième ne donnant jamais d'ascospores ; cette race asporogène s'est maintenue très longtemps en cet état; HaNSEN n'a pu faire renaître en elle la faculté de donner des spores qu'en la cultivant dans du moût de bière additionné de glucose. Néanmoins, la variation est réversible et il n'y a pas à proprement parler mutation, c'està-dire production d'espèce ou de sous-espèce nouvelle.

Bien qu'il y ait quelque difficulté à préciser les analogies de ces variations avec les modifications de même ordre chez les végétaux supérieurs, je vais indiquer plusieurs faits qui fournissent, sur ces états stabilisés mais non définitifs, une idée assez claire du phénomène.

Ises pépiniéristes multiplient souvent de bouturages l'espèce à feuillage vert et les variétés à feuilles panachées du Troène (Iigustrum vulgare L.), dont on fait des haies supportant la taille. Ils ont reconnu que les bontures prises sur les 
pousses inférieures, qui ne porteront des grappes de fleurs que deux ou trois années plus tard, donnent, presque toujours, d'u plant vigoureux à vieillissement, c'est-à-dire à floraison et à fructification tardives, par exemple dix ans après le bouturage ; que les boutures prises dans le haut des pieds mères, au voisinage des grappes fleuries, donnent un plant à entrencuds courts portant deux ou trois ans après, ou l'année suivant le bouturage, des pousses fleuries et fructifiées. Ainsi, on obtient à volonté deux séries d 'individus à partir d'une même plante, c'est-à-dire de la même lignée pure ; elles représentent deux phases distinctes qui se propagent à l'infini en conservant leurs caractères, et cela, semble-t-il, indépendamment du milieu ; il n'y a évidemment pas mutation.

Mais le milieu, cause de cette adaptation double, est ici le mouvement particulier de la sève et l'élaboration locale de la sève qui font que sur le même arbaste il y a des bourgeons à bois et des bourgeons (ou boutons) à fleurs. L'impulsion première donnée par la diversité des positions, e'est-àdire par la nutrition des bourgeons, peut, chez la plupart des espèces végétales, être perpétuée indéfiniment, lorsqu'on maintient les bourgeons déjà différenciés dans un milieu favorable à la croissance d'un des deux états ; le retour on la mort ne sont obligatoires que si les milieux limitent ou ne permettent pas le développement des tissus caractéristiques des deux phases Il y a dans ces faits beaucoup de points communs avec les adaptations doubles de la Renouée amphibie.

Il semble que les prétendiues variations provoquées et perpétuées par la greffe sont de cette nature. La greffe multiplie les états, mais n'empêche pas, en définitive, l'individu de vieillir. Je conserve avec soin une collection de Poi- 
riers, la plupart dérivés d'individus appréciés au cours du xvIII $^{\mathrm{e}}$ siècle; sur cent dix sortes, je n'ai récolté des fruits en dix ans que sur trois sortes (individus) et une vingtaine seulement donnent des fleurs. Ils n'auraient guère été estimés il $\mathrm{y}$ a cent cinquante ans s'ils avaient présenté les mêmes tares que j'attribue à la vieillesse des individus, et mes efforts pour les rajeunir par des greffes sur des sujets divers, cognassiers, poiriers, aubépines, ont jusqu'à présent échouté.

Ces faits n'excluent pas d'ailleurs la possibilité d'une multiplication indéfinie d'un même être sous deux états parallèles, qui même peuvent être maintenus par le semis. J'ai signalé ailleurs (1) les procédés qui permettent la perpétuation par voie de semis des formes juvéniles du Chanvre, et j'ai montré que, chez les Thuya, une forme juvénile à feuilles aciculaires, comme celles du Génévrier, se maintient par le semis et a été propagée sous le nom de genre Retinospora. Il n'y a dans ces deux exemples, d'ailleurs exceptionnels, qu'une extension du phénomène de paedogénèse découvert, il y a une trentaine d'années, chez l'Amblystome du Mexique ; ce lézard se propage, dans certaines localités du Mexique, aussi bien par la reproduction de la larve (Axoloth) que par la reproduction de l'adulte (Amblystome).

De même, on ne considère pas comme des mutations vraies, c'est-à-dire des changements d'espèce, les formes fixées si nombreuses actuellement de plantes, d'insectes, de crustacés, qui se reproduisent régulièrement par parthénogénèse. Ce sont des états d'une espèce qui ne présentent qu'une seule phase dans un cycle complexe parcouru habituellement

1. - Sur la production des formes infantiles. Revue d'histoire naturelle appliquée, juin 1922. 
par l'espèce sauvage. Nous revenons ainsi, presque naturellement, à l'observation de PASTEUr, qui compare les diverses levures sélectionnées par l'homme à des états particuliers des plantes cultivées, tellement différents des prototypes sauvages qu'on ne sait établir leurs affinités. Nous allons voir qu'après avoir admis, sous l'impulsion des idées régnantes, de semblables métamorphoses, PASTEUR arrive à en nier la possibilité. Les progrès qu'il a provoqués dans l'art des fermentations, dans la préparation des vaccins, reposent sur une idée fondamentale : la spécificité absolue et inébranlable des ferments figurés et des microbes pathogènes. 


\section{ADAPTATIONS RÉCIPROQUES ET VACCINS}

E. Duclaux (1), qui fut le collaborateur assidu de PasTEUR pour toutes les recherehes sur la fermentation, nous conte, avec humour, comment son Maître, après avoir combattu, dès 1861, la transformation des espèces microscopiques les unes dans les autres, s'était peu à peu laissé entraîner par l'éelat des idées nouvelles développées par DARwin et l'école évolutionniste. "Ce n'étaient pas, à coup sûr, les démonstrations de BalL, de Hoffrians, ni même celles de Trécul, qui pouvaient donner eréance à ces idées. Tous ces botanistes éminents étaient de pauvres expérimentateurs, allant pour ainsi dire au-devant des causes d'erreur, non pour leur barrer la route, mais pour leur livrer carrière. " Mais PASTEUr avait une foi absolue en l'expérienee, et voici ce qu'elle lui montrait.

Le Mycoderma vini, ou fleur du vin typique, est semé sur du moût de bière étalé dans une assiette plate; il forme bientôt un voile à la surface. PASteur immerge le voile, après l'avoir fortement agité dans le liquide pour en mouiller les parties ; il introduit le tout dans un flacon choisi de telle sorte qu'il ne reste plnıs d'air après l'avoir fermé par un bouchon muni d'un tube coudé débouchant sous l'eau. Dans

1. - Histoire d'un Esprit, p. 241. 
le flacon clos commence bientôt une fermentation véritable que Pasteur attribuait aux cellules du myeoderme, devenues cellules de levure de bière. L'expérience réussissait de même lorsque le liquide nutritif employé était du vin sucré et dilué d'eau.

" A Clermont, dit Duclaux, où il me faisait l'honnenr de travailler dans mon laboratoire (1870-1871), nous avions fait plusieurs fois cette expérience, et comme, étant son élève, j'étais naturellement plus intransigeant que lui, je refusais de me rendre à cette preuve, et j'objectais la présence possible de globules de levure tombés de l'air ou venus de l'eau ou des vases avant ou après le transvasement, malgré les précautions prises pour les éviter. PASTEUR résistait parce que, dans son esprit, ce fait se rattachait à d'autres notions... relatives à la théorie générale de la fermentation. L'expérience, du reste, réussissait parfois avec une netteté qui la rendait probante et me fermait la bouche. Bref, PASTEUR avait rapporté sa conviction à Paris, et on la voit reparaître à plusieurs reprises dans ses notes de 1872 et 1873 à l'Académie des Sciences. »)

La levure de bière est formée de cellules plus grosses, plus rondes que celles du Mycoderma vini, mais PASTEUR avait été frappé par les transformations de l'Aspergillus dans le travail de VAN TIEGHeM, et lui-même avait suivi les modifications de formes que le Mycoderma vini était susceptible de prendre dans des conditions variées. Néanmoins, la métamorphose lui paraissait suspecte et, après bien des tâtonnements, il réussit à établir qu'elle n'a pas lieu.

En répétant l'expérience, il trouve un jour au fond des flacons où il avait submergé la fleur de vin de grosses cellules sphériques rondes du Mucor racemosus (p. 175), et cette fois trop différentes du Mycoderma vini pour qu'il y 
eût possibilité logique d'une mótamorphose aussi radicale. "Puisqu'il y a du mucor mucedo ou racemosus, quoique je n'aie semé que du mycoderma vini, se dit PASTEUR, e'est done qu'une ou plusieurs spores de ee mucor ont été apportées par l'air ambiant. Or, si l'air apporte des spores de muсо dans mes opérations, pourquoi n'apporterait-il pas des eellules de levure, dans mon laboratoire surtout ? »

En outre, dans la série des nombreuses expérienees, quelques-unes eurent un résultat négatif ; " la transformation du mycoderme en leviure n'y eut pas lieu, quoique les conditions fussent aussi semblables qu'on pouvait le souhaiter à celles des expériences où je la voyais réussir ; comment admettre que, parmi les milliards de cellules submergées, toutes fussent impropres à la transformation, si cette transformation était réellement possible ? »

PASTEur examine done les eirconstanees an cours des manipulations qui favorisent l'introduction aeeidentelle de germes de levures; il résoud la question en imaginant le flacon à deux tubulures déerit plus hant (page 187), et en l'utilisant pour la préparation des cultures pures du Myco. derma vini. "Plus jamais, ajoute PASTeUn, je ne revis de la levure, ni de fermentation aleoolique aetive, à la suite de la submersion des fleurs, soit dans les ballons mêmes, soit dans les matras d'essayeur " où il transversait aseptiquement les liquides pour réduire au minimum leur surface de contaet avec l'air.

La notion de spéeificité des levures était sauvée. Ces tâtonnements, ces illusions détruites après tant de réflexion et avec tant d'habileté, firent plus que le raisonnement; ils déeidèrent PASTEUR à nier la transformation possible et actuelle des espèces les unes dans les autres. Il faut avouer que les expériences réalisées depuis ont confirmé son point 
de départ et ses conséquences ; le transformisme des espèces de microorganismes reste douteux.

Le voilà armé pour l'étude des maladies microbiennes, dont il avait à diverses reprises signalé l'analogie avec les phénomènes de la putréfaction et de la fermentation. Il connaissait dans leurs moindres détails les phases successives de la lutte pour la vie des microorganismes dans un milieu limité ; il savait par RAULIN l'importance de la plus légère altération des solutions nutritives; il était en quelque sorte l'inventeur de la notion de vie anaérobie; il croyait à l'immuabilité de l'espèce dans les conditions ordinaires de l'expérimentation. En quelques années, il précise les causes de plnsieurs maladies infectieuses et découvre une méthode générale de préparation des vaccins.

$$
\text { *** }
$$

Bien que les conceptions originales et la technique de PASTEur relatives à la production et à l'utilisation des vaccins pour la lutte contre les maladies contagieuses soient le résultat des travaux relatifs à la Bactéridie charbonneuse, c'est surtout dans l'étude du Choléra des poules qu'il laisse apparaître à la fois l'audace de sa pensée et la rigueur des déductions qui, vérifiées par des séries de faits choisis, aboutissent à la certitude scientifique.

Les comptes rendus de l'Académie des Sciences (1880) fournissent sur l'état d'esprit de PASTEUr à cette époque un important témoignage : "Je prends la liberté, dit-il, de rappeler à mon confrère, M. Blanchard, que les illusions d'un expérimentateur sont une grande partie de sa force. Ce sont les idées préconçnes qui lui servent de guide. De celles-ci, beaucoup, le long du chemin qu'il parcourt, s'éva- 
nouissent. Nais, un beau jour, il reconnaît et il prouve que quelques-mes d'entre elles sont adéquates à la vérité. Alors il se trouve maître de faits et de principes nouveaux dont les applications, tôt ou tard, répandent leurs bienfaits. "

Duclaux, dans l'Histoire d'un Esprit (page 340), nous fait connaître quà cette même époque PASTEur était sous l'influence profonde de la lecture des travaux de Jenner relatifs à la vaccination contre la variole. D'ordinaire, sur l'homme, la variole exerçait des ravages redoutables, et les épidémies les plus connues étaient meurtrières; parfois, il $\mathrm{y}$ en avait de bénignes; on savait aussi que la maladie, grave an début de l'épidémie, diminuait en général de virulence à meșure que l'épidémie tirait à sa fin. D'autre part, il n'était plus douteux pour Pasteur que le passage du vacein de la vache à l'homme rendait ce vaccin inoffensif, lors même qu'on le communiquait de bras à bras durant une longue suite de générations.

Le contraire avait lien pour la Bactéridie charbonneuse. En 1869, Coze et Feltz avaient noté, puis Davaine avait confirmé que le virus angmentait de force en passant par l'organisme; alors que le sang du premier animal inoculé n'était mortel qu'à la dose d'un dixième de goutte, par des passages, e'est-à-dire après des inoculations successives et rapprochées sur des animaux frais, la dose mortelle tombait à un centième, à un millième de goutte. Si PASTEur avait vécu isolé comme JokdAN, s'il n'avait pas pris pour règle d'abandonner ses idées préconçues devant les expériences décisives, il anrait trouvé dans ces faits les caractères de mélanges de microbes ou de modificatious indépendantes de ceux-ci ; il n'aurait peut-être pas déconvert le principe de la préparation des vaccins. 
L'étude du Choléra des poules, puis du Charbon, força pour ainsi dire PASTEUr à accepter la notion, si familière à présent, de l'atténuation des virulences d'un même microbe, et lui prouva, par surcroît, qu'il y a des maladies à microbes qui ne récidivent pas.

"Parfois se déclare dans les basses-cours une maladie désastreuse qu'on désigne sous le nom de Choléra des poules. L'animal en proie à cette affection est sans force, chancelant, les ailes tombantes. Les plumes du corps, soulevées, lui donnent la forme en boule. Une somnolence invincible l'accable. Si on l'oblige à ouvrir les yeux, il paraît sortir d'un profond sommeil. Bientôt, les paupières se referment, et le plus souvent la mort arrive sans que l'animal ait changé de place, après une muette agonie. C'est à peine si, quelquefois, il agite les ailes pendant quelques secondes (1). ")

Prélevant une goutte de sang dans les muscles, dans la erête, dans les veines ou les artères, PASTeur l'ensemence dans des tubes de culture stérilisés renfermant quelques centimètres cubes de bouillon de poule neutralisé ; dans tous les cas, il s'y développe un microbe formé d'articles immobiles, légèrement étranglés en leur milieu et de dimensions très faibles. Pasteur hésite à les appeler bacilles et admet que leur forme étranglée indique ume phase de bipartition ; ce sont pour lui des Micrococcus de 0,2 à 0,3 millièmes de millimètres d'épaisseur ; souvent, le dépôt qu'ils forment sur' les parois et le fond des tubes de culture est invisible; ils sont cependant retenus par les filtres, et le bonillon, cultivé, puis filtré, reste inoffensif. L'existence de ces microbes fut d'abord soupçonnée par M. MoRITz, vétérinaire dans la

1. - L. Pasteur : Sur les maladies virulentes et en particulier sur la maladie appelée vulgairement choléra des poules. C. R. Ac. des Sciences, 1880, xc, pp. 239, 952 et 1030. 
Haute-Alsace ; MI. Peroncito, de Turin, les avait figurés en 1878 , et M. Toussaint, professeur à l'Ecole vétérinaire de Toulouse, venait de faire connaître à l'Académie des Sciences (1880) que ces petits organismes étaient bien la cause de la virulence du sang des animaux atteints de choléra.

La poule n'est pas la seule volaille atteinte; les canards, d'ordinaire si résistants, les oies, les dindons, les faisans, les pigeons surtout, $y$ sont sujets, et aucune race ne paraît épargnée ; mais les jeunes poulets y succombent, en général, par masses et le nom Choléra des poules fut définitivement admis. Le chien, le cheval, peuvent héberger le microbe ; les moineaux le propager; le lapin y est sensible, et PASTEUR reconnut que la contagion peut s'étendre de terriers à terriers. Le cobaye résiste aux inoculations sous la pean; l'abcès guérit rapidement sans que l'animal semble malade : "Des poules ou des lapins qui vivraient en compagnie de cobayes portant de tels abcès pourraient tout à coup devenir malades sans que la santé des cochons d'Inde parût le moins du monde altérée. Pour cela, il suffirait que les abcès des cochons d'Inde, venant à s'ouvrir, répandissent un peu de lear contenu sur les aliments des poules et des lapins... Combien de mystères dans l'histoire des contagions, ajoute PASTEUR, recevront un jour des solutions encore plus simples que celle dont je viens de parler ! " (9 février 1880.)

Le germe défini, il fant l'étudier sur des milieux stériles, et, de suite, PASTEUR éprouve des surprises qui linvitent à un examen approfondi. En bouillon de muscles de poule, neutralisé par la potasse et stérilisé entre 110-115 degrés, la multiplication tient du prodige; en quelques heures, le bouillon le plus limpide devient trouble. Mais une décoction de levure de bière, rendue parfaitement limpide par filtra- 
tion et stérilisée par la chaleur ne lui convient pas du tout ; en moins de quarante-luuit heures, le microbe périt, fait étonnant, car ce milieu convient à des microorganismes les plus divers. Pasteur note en passant que cette propriété de l'eau de levure fournit un moyen précieux pour s'assurer de la pureté des cultures du Micrococcus du Choléra des Poules; l'eau de levure limpide ne se troublera que si le Micrococcus ensemencé est accompagné d'autres organismes. Par contre, le bouillon de muscles de poule paraît le milieu le plus convenable à la fixation de sa virulence et de son développement : en passant d'une culture à la suivante par l'ensemencement d'une quantité pour ainsi dire infiniment petite en tubes stérilisés de bouillon de poule neutralisé et filtré, le Micrococcus détermine toujours les mêmes troubles caractéristiques dans les bouillons limpides ; il se multiplie toujours avec autant de facilité lorsqu'on l'inocule à des Gallinacés, lesquels, vingt fois sur vingt, périssent en deux ou trois jours et, le plus souvent, en moins de vingt-quatre heures. Pasteur possède donc deux milieux de culture à effets extrêmes et opposés sur la multiplication du microbe ; les circonstances inattendues de l'atténuation vont maintenant se présenter avec les caractères d'expériences décisives.

" Par certain changement dans le mode de culture, on peut faire que le microbe infectieux soit diminué dans sa virulence... ; elle se traduit dans les cultures par un faible retard dans le développement du microbe ; mais, au fond, il y a identité de nature entre les deux variétés du virus. Sous le premier de ses états, l'état très infectieux, le microbe inoculé peut tuer vingt fois sur vingt. Sous le second de ses états, il provoque vingt fois sur vingt la maladie et non la mort. Ces faits ont une importance facile à comprendre ; ils nous permettent, en effet, de juger, en ce qui concerne la 
maladie qui nous oceupe, le problème de sa récidive ou de sa non-réeidive. Prenons quarante poules, inoculons-en vingt avec un virus très virulent ; les vingt ponles mourront. Inoculons les vingt autres avec le virus atténué, toutes seront malades, mais elles ne mourront pas. Laissons-les se guérir et revenons, ensuite, pour ces vingt poules, à l'inoculation du virus très infectieux; cette fois, il ne tuera pas. La conclusion est évidente; la maladie se préserve elle-même; elle a le caractère des maladies virulentes, maladies qui ne récidivent pas. ")

Le fait nouvear n'est pas l'absence de récidive; la vaccination est connue dans ses effets depuis longtemps. Mais les virus vaccins, de même que les maladies qu'ils atténuent dans leurs conséquences, sont inconnus dans leur nature propre. Dans le cas du Choléra des poules, l'agent est un parasite microscopique, un être vivant cultivable hors de l'économie. A une certaine phase de son traitement, c'est-àdire de son atténuation, il se comporte comme un vaccin relativement à celui qui tue. Il reste à savoir si le microbe devenu vaccin est fixé dans sa variété propre, s'il persistera dans sa forme atténnée, s'il ne faudra pas toujours recourir à sa préparation d'origine chaque fois qu'on voudra en faire usage. »

$$
* *
$$

Le problème posé ainsi en 1880 par L. Pasteur ne diffère pas dans son essence de la question de l'hérédité des caractères acquis qui, à la même date, était au premier plan des préoccupations des biologistes. DaRwis énonce le problème d'une manière vague, mais qui est précisément celle généralement adoptée :

" Il est probable qu'un grand nombre de déviations de 
structure, qui apparaissent dans les produits de génération asexuelle, sont le résultat de l'action directe des changements de conditions sur l'organisation, indépendamment des organes reproducteurs. Dans quelques cas, nous sommes à peu près certains qu'il doit en être ainsi lorsque tous ou presque tous les individus qui ont été exposés anx mêmes conditions se trouvent affectés d'une manière définie et semblable, comme les Maïs importés de pays ehauds et cultirés en Allemagne, les modifications survenues dans les toisons des moutons sous les tropiques, l'augmentation de taille et la précocité de nos races domestiques les plus améliorces, l'hérédité de la goutte à la suite d'excès, et un grand nombre d'autres cas. Or, des changements de ce genre n'affectent pas d'une manière spéciale les organes reproducteurs; il semble étrange que leur produit, le nouvel être organisé, se trouve en fait affecté d'une manière semblable (1). "

A la vérité, plusieurs des faits invoqués par DARwin ont reçu une solution qui ne laisse au climat qu'un rôle éliminateur. Les fameux Maïs blanes Dent de Cheval de Metzger, devenus quelques années plus tard Maïs à petits grains jaunes, étaient incontestablement le résultat de la féconda. tion, grâce au vent, des ovaires de cette sorte par le pollen des variétés eultivées dans l'Allemagne du Sud ; e'est une substitution de germes du type exposé plas haut (p. 106). La même objection ne peut être opposée aux modifications a.cquises par les Céréales du Centre de l'Europe, transportées en Suède, qui y acquièrent une maturation plus rapide et des grains plus lourds. Ici, il s'agit de plantes autofé. condées, et de plus, la variation est réversible, e'est-à-dire

1. - Variations des animaux et des plantes, trad. Moulinié, t. II, p. 395 . 
que les formes à car'actères nouvearux, cultivées dans l'Europe centrale, y reprennent les caractères ancestraux en quelques générations.

Remarquons d'ailleurs que les essais réalisés avec des lignées pures pédigrées depuis le développement de l'Institut des Semences de Svalöf ont réduit à des variations moins marquées, quoique sensibles encore, les modifications acquises par les Céréales strictement autofécondées et dérivées d'un seul grain. J'en ai observé de nombreux exemples à la suite des introductions répétées de l'Orge Prinzess de Svalöf, et surtout de l'Orge Hannchen qui, l'année de leur importation de Suède en diverses contrées de France, se distinguent nettement des mêmes lignées pures introduites du même pays, mais cultivées en France depuis dix ans. Ce qu'on peut affirmer aussi, e'est que les différences s'atténuent rapidement et deviennent insensibles au bout de trois à quatre années de cultures parallèles dans les mêmes centres.

Mais je ne veux retenir ici l'attention que sur un fait. D'après DARwin, dans la phrase citée ci-dessus, et surtout d'après les partisans de l'Ecole de Weismann, qui a limité, sans les annihiler totalement, les effets héritables dus aux changements de milieux et de climats, les organes sexuels, les éléments reproducteurs, ne paraissent pas touchés par ces alternatives. Que penser alors de la fameuse expérience de Darwin et de Fritz Muller avec le Pavot jaune de Californie (Eschscholtzia californica) ? Au Brésil, où Fr. Muller l'étudia, cette plante est autostérile, le pollen propre ne faisant pas nouer les fleurs en fruits ; des graines furent envoyées à DARwin, qui en fit l'épreuve en Angleterre, et là les mêmes lots donnèrent par autofécondation un assez grand nombre de graines durant quelques années. Renvoyés 
à Fritz Mulier an Brésil, ils s'y comportèrent comme le point de départ, ne donnant pas de graines par autofécondation. Il y a done une influence bien marquée du climat sur les affinités relatives des pollens et des ovules, et je me suis assuré sur l'Eschscholtzia lui-même et sur diverses lignées d'Orges, de Blés, de Lins, que ce processus est fréquent ; il constitue même nun moyen commode pour s'assurer de l'instabilité plus ou moins grande des lignées pures cultivées.

Au surplus, Galton (1), neveu de Darwin, a montré par des déductions logiques saisissantes que, dans les lignées pures, I'accumulation des déviations sous l'influence des conditions de uutrition étaient limitées. Par un tout autre procédé que PASTEUR, il arrive à fournir sur les oscillations des caractères variables et sur leur réversibilité des aperçus qui confirment le point de vue de PASTEUR, à savoir qu'on ne provoque point, par les procédés de culture, des altérations irréversibles des qualités propres à l'espèce pure.

$$
*
$$

Une expérience réalisée par L. PASTEUR vers la même date montre un tout autre aspect du phénomène. La distinction des idées qui dominent l'orientation des recherches est souvent difficile dans les expériences pastoriennes, parce que la sécurité obtenue par la préparation de cultures pures, par les connaissances acquises en quelques jours sur la valeur relative des milieux nutritifs, ne laissent pas le temps de s'accrocher à des suggestions erronées ; alors qu'il fallut trente années pour confirmer les vues de GALTON relatives à la

1. - Voir L. Blaninghem : Les problèmes de l'hérédité expérimentale, 1919, chap. II. 
réversibilité des modifications dues au milieu et aceentuées par la sélection méthodique, la eonception d'une altération définitive du mierobe qui, daus le eas de la vaceine de la variole, paraissait nettement établie, ne pouvait longtemps résister à des épreuves expérimentales sur des êtres vivants parfaitement définis.

Le microbe du Choléra des poules est si ténu, que parfois le dépôt qu'il laisse au fond et sur les parois des tubes de bouillon de muscles de poule alcalinisé reste invisible. Il ne paraît même pas altérer le bouillon, si l'on néglige la phase de trouble qui suit de vingt-quatre à quarante-huit heures son ensemencement. Pasteur eonstate cependant que l'altération est profonde ; filtré au travers d'une cloison poreuse, il y abandonne tous les éléments figurés et reste indéfiniment stérile et inactif après inoarlation; or, le filtrat est ineapable de servir de milieu nutritif à un ensemencement de microbes virulents ou atténués du Choléra des poules. Le premier ensemencement, suivi d'une aetive multiplieation du microbe, même de contrte durée, rend le milieu impropre à un second ensemencement, de même qu'une première attaque de variole protège eontre nue nouvelle atteinte. La vacciuation n'est peut-être pas due à une altération profonde du mierobe lui-même, mais sans doute à une réaction de l'organisme sounis à une première infection.

PASTEUR s'explique alors diverses exeeptions notées au cours de ses expériences préliminaires. L'inoculation du microbe virulent du Choléra aux Gallinaeés est presque toujours suivie d'une mort rapide, mais il arrive parfois que la poule, après avoir été plus ou moins gravement malade, donne lieu à des espoirs de guérison; elle mange. peu, elle maigrit, puis finit par suceomber après des semaines et des mois de langueur. De mêne, dans certaines maladies 
virulentes, rougeole, scarlatine, fièvre tyṕhoïde, l'évolution fatale et rapide est parfois retardée ; les maladies épidémiques graves présentent de temps à autre des formes larvées.

Le 3 mai 1880, L. PASTEur apporte l'explication décisive à l'Académie. Lorsqu'on injecte sous la peau d'une poule neuve en très bonne santé l'extrait d'une culture filtrée de Choléra des poules correspondant à un développement très abondant dn microbe, la poule présente durant un quart d'heure des désordres nerveux qui se dissipent peu à peu; elle prend encore la forme en boule, reste immobile, refuse de manger, éprouve une tendance marquée au sommeil, présente en fait tous les symptômes précurseurs d'une maladie violente, comme dans le cas des maladies provoquées par l'injection du microbe virulent. Mais ces accidents, atténués en ce sens que le sommeil est plus léger, ne persistent pas ; après quatre heures environ de troubles caractérisés, la poule redevient alerte, mange et glousse comme avant l'injection.

Il est done probable que le parasite fabrique, au cours de sa multiplication intense dans le milieu de eulture, des substances narcotiques dont la présence en quantité limitée dans l'organisme de la poule neuve suffit à provoquer, pour un temps limité lıi aussi, le symptôme si prononcé de somnolence et d'apathie caractéristique de l'infection par le choléra lui-même.

En ce dernier cas, la mort de l'animal est due à la fabrication continue du narcotique par le microbe qui pullule; de plus, l'avidité du microbe pour l'oxygène accélère ou détermine la mort; il y a ici lutte entre le microbe et l'organisme pour un élément essentiel à la vie, l'oxygène.

S'il avait suivi ces deux faits distinets, PASTEUR aurait 
sans aucun doute réalisé de suite les immenses progrès faits par ses élèves et ses émules, relatifs à l'immunité humorale. Il $n$ 'y revient que plus tard, lorsqu'il entendit parler des travaux de MeTchNizoff sur la phagocytose, et tout de suite il y prêta la plus grande attention. En chimiste habitué à la permanence des états, puisque cette science est ou parut être au cours du siècle dernier purement statique, Pasteur ne pouvait concevoir d'emblée les réactions réciproques et parallèles des organismes en présence. Mais il était familiarisé par ses études sur les fermentations avec la lutte d'organismes divers pour l'utilisation d'un aliment, et il admit presque tout de suite la conception si simple du physiologiste russe. Les globules blanes du sang et des tissus s'emparant des microbes plus petits, moins actifs, les englobant pour les digérer et les faire disparaître, était une notion certainement étrangère à la folmation intellectuelle de PASTEUR; elle lui plut, comme l'a fait remarquer Duclaux, parce $q u$ 'elle égalisait les forces en présence; de petits globules en lutte avec de très petits microbes donnaient au phénomène de la résistance une allure plus simple, quoique au fond aussi complexe, que celle d'une lutte entre l'organisme poule ou l'organisme bouf et un parasite dont les dimensions $\mathrm{n}$ 'atteignaient pas un millième de millimètre. Les théories de l'immunité découlent des travaux de Pasteur, elles ne lui appartiennent pas.

\section{***}

Mais ce qui lui appartient bien et suffit à révolutionner les sciences médicales, c'est la conception d'une méthode générale de préparation des vaccins contre les maladies infectieuses. PAsteur avait à la fois le scns le plus profond de l'analyste dans l'examen des cas particuliers, découvrant 
les plus infimes détails, leurs liaisons, leur importance et, lorsqu'il avait éclairci ces liaisons, vérifié leurs conséquences, avec une audace inouie résultant d'une confiance absolue en la simplicité et l'unité des lois de la nature, il généralisait, embrassait dans une conception d'ensemble tous les faits analogues, sonvent même éloignés.

Une circonstance imprévue, qui risquait de retarder ses études sur le Choléra des poules, le mit sur la voie. Pendant les vacances de 1879, presque toutes les cultures artificielles du Micrococcus laissées au laboratoire avaient perdu leur vitalité. " Comme elles correspondaient toutes, dit DucLAux (Histoire d'un Esprit, p. 348), à des expériences en train, on avait essayé de les revivifier, et pour cela on leur avait emprunté des semences qu'on avait fait passer, soit par des bouillons de poule, soit par des poules. Beaucoup n'avaient pas donné de culture, avaient aussi respecté et laissé intacts les animaux inoculés, et on était au moment de tout jeter, pour tout recommencer à nouveau, lorsque Pasteur eut l'idée de faire inoculer une culture jeune et neuve, à ces poules qui avaient si bien supporté, du moins en apparence, les inoculations des cultures de l'été précédent.

" A la surprise de tous, peut-être même celle de PASTEUR qui ne s'attendait pas à pareille réussite, presque toutes ces poules résistèrent, alors que des poules neuves et venant du inarché succombaient dans les délais ordinaires, montrant ainsi que la culture inoculée était très active. Du même coup, le Choléra des poules passait an cadre des maladies virulentes et la vaccination était trouvée ! "

Le 26 avril 1880, L. PASTEur expose les résultats décisifs à l'Académie ; sur vingt poules inoculées par le virus très 
actif, aucune ne survit. Mais vingt poules inoeulées par une seule piqûre du virus le plus attéuué survirent toutes ; piquées ensuite arec le virus très actif, six ou huit résistent et, par conséquent, sont vaceinées par le virus très actif. Deux piqûres successives, trois, quatre, ete., placent graduellement l'animal dans des conditions telles qu'il ne contractera plus, sans aucune exception, le Choléra des poules.

Ainsi, il est pronvé que c'est la vie, la pullulation d'un parasite à l'intérieur du corps qui détermine la maladie appelée vulgairement Choléra des poules. Du moment où cette culture n'est plus possible dans la poule, la maladie ne peut apparaître; les poules sont alors dans l'état constitutionnel des animaux que le Cloléra n'atteint jamais.

Il n'y a pas lieu de trop s'étonner qu'il existe des constitutions tantôt aptes, tantôt rebelles aux inoculations, lorsqu'on voit le bouillon de levure de bière, préparé exactement comme le bouillon de muscles de poule, se montrer absolument impropre à la vie du microbe.

La cause de la non-récidive du Choléra très actif inoculé dans une poule préalablement inoeulée par le même Choléra peu actif, et qui a résisté à cette première infection, doit se trouver dans le fait que le microbe, même pen actif, altère ou détruit certaines matières du corps de l'animal, soit qu'il les élabore à son profit, soit qu'il les brûle par l'oxygène qu'il emprunte au sang.

Pasteur étudie le mécanisme de cette résistance. Il inocule une culture du Micrococcus en bouillon de poule ayant une quinzaine de jours; il constate que la virulence, qui était totale dans les premiers jours, diminue; toutes les poules sont malades, mais toutes ne meurent pas. La piqûre 
est faite dans le muscle pectoral, par exemple ; le muscle se tuméfie, durcit et blanchit dans toute sa masse, et on peut vérifier qu'il est complètement infiltré de microbes, lesquels d'ailleurs présentent une forme différente de celle dn sang et paraissent plus longs. C'est pourquoi, à la suite de cette constatation, le microbe fut appelé Bacillus cholerce gallinorum, la forme allongée étant plus caractéristique et plus constante que la forme ronde; au début des recherches, on l'avait attribuée à une phase de division des Micrococcus ; sa constance dans les milieux de culture divers justifie la nouvelle dénomination. Hais ce n'est là qu'un point de détail. Les Bactéries se sont multipliées dans tout le inuscle, qui devient lardacé avec trainées de globules de pus, sans d'ailleurs fournir de suppuration massive et localisée; le muscle est profondément altéré; ses fibres sont désagrégées et se dissocient sous la moindre pression et, le processus continuant, la mort lente s'ensuit.

Or, certains animaux résistent; la portion musculaire désagrégée se ramasse, durcit, forme un îlot dans une cavité dont les parois présentent bientôt l'aspect d'une plaie profonde en voie de guérison. Le massif musculaire nécrosé se résorbe en quelques mois ; d'ailleurs, on peut l'extraire par une légère incision sans modifier le processus général et on hâte la gnérison par cette intervention.

Ainsi, la maladie provoçuée par le Bacille atténué présente nne érolution toute différente de la maladie résultant de l'inoculation ou de l'ingestion d'un Bacille très vimulent. Ici il y a infection généralisée; le microbe a envahi le sang et tous les organes. La propagation du Bacille atténué est lente ; les symptômes de somnolence, d'abattement, sont les mêmes an début; mais le parasite reste localisé ; il détruit une partie du muscle, lequel ne se laisse pas envahir et 
guérit en laissant à l'envahisseur une fraction plus ou moins grande de sa substance. Les modifications cellulaires qui accompagnent la lutte ont été, peu de temps après, clairement mises en relief par Metchniroff, et on conçoit avec quelle attention PASTEUR a suivi, dès qu'il en eut connaissance, les travaux de l'éminent savant russe qui devint bientôt son collaborateur.

$$
\text { *** }
$$

Le 26 octobre 1880, L. PASTEUR fournit des renseigncments décisifs sur les causes immédiates de l'atténuation du Bacille du Choléra des poules et, par voie de conséquence, sur la production expérimentale des vaccins. Il présente sa démonstration à l'Académie des Sciences avec un soin tout particulier, ayant déjà entrevu les magnifiques applications de sa découverte à la lutte contre les épidémies infectieuses. Elle comprend trois points essentiels où l'on retrouve les certitudes acquises dans les chapitres précédents et des conséquences pratiques importantes en rapport avec les faits d'adaptation qu'il observe.

" Le moment est venu de m'expliquer sur l'assertion capitale..., à savoir qu'il existe des états variables de virulence dans le choléra des poules; étrange résultat, assurément, quand on songe que le virus de cette affection est un organisme microscopique qu'on peut manier à l'état de pureté parfaite, comme on manie la levure de bière ou le mycoderme du vinaigre...

"Dans le cas particulier qui nous occupe, le mystère apparaît surtout dans cette circonstance que, le virms étant un parasite microscopique, les variations dans sa virulence sont à la merei de l'observateur..... 
" C'est l'oxygène de l'air qui affaiblit et éteint la viru. lence.

"Vraisemblablement, ajoute Pasteur, il y a ici plus qu'un fait isolé ; nous devons être en possession d'un principe. On doit espérer qu'une action inhérente à l'oxygène atmosphérique, force naturelle toujours présente, se montrera efficace sur les antres virus. C'est, dans tous les cas, une circonstance digne d'intérêt que la grande généralité possible de cette méthode d'atténuation de la virulence, qui emprunte sa vertu à une influence d'ordre cosmique en quelque sorte. »

La démonstration de l'atténuation de virulence du microbe du Choléra des poules devait avoir lieu avec une rigueur décisive. Pasteur met tout en œuvre pour en faire l'expérience irréfutable. Il a reconnu que le virus prélevé dans le corps d'une poule qui vient de mourir, non de la maladie aiguë, mais de la maladie chronique, est le plus virulent; les cas en sont rares; la poule est très malade, maigrit de plus en plus et résiste à la mort pendant des semaines et des mois. "Lorsqu'elle périt, ce qui a lieu peu de temps après que le parasite, localisé jusque-là dans certains organes, a passé dans le sang et s'y cultive, on observe que, quelle qu'ait été la virulence originelle du virus au moment de l'inoculation, celui qu'on extrait du sang de l'animal qui a mis un si long temps à mourir est d'une virulence considérable qui tue ordinairement dix fois sur dix, vingt fois sur vingt.

“ Cela posé, faisons des cultures successives de ce virus, à l'état de pureté, dans du bouillon de muscles de poule, en prenant chaque fois la semence d'une culture dans la culture précédente, et essavons la virulence de ces cultures 
diverses. L'observation démontre que cette virulence ne change pas d'une manière sensible. " Autrement dit, l'inoculation de ces divers états du virus au même nombre d'animaux de même espèce entraîne la même proportion de mortalité dans le même temps.

Préparons maintenant une quantité convenable de bouillon de poule, que nous ensemençons avec le virus très virulent, et répartissons-la dans des tubes de verre de même taille que nous remplissons aux deux tiers, anx trois quarts de leur volume, etc., avant de les fermer à la lampe. La petite quantité d'air présente dans les tubes suffit pour permettre le développement du virus, qu'on recomnait au trouble croissant du liquide; tout l'oxygène des tubes disparaît en deux ou trois jours; alors le trouble tombe, le liquide s'éclaircit, le virus se dépose sur les parois, et il reste désormais à l'abri du contact de l'oxygène jusqu'au jour où l'on ouvrira les tubes. Ouvrons-les, l'un après un intervalle $d$ 'un mois, et, après avoir fait une culture par ensemencement d'une portion de son contenu, essayons-en la virulence ; l'autre après un intervalle de deux mois, et ainsi de suite pour un troisième, un quatrième tube, etc., après des intervalles de trois à dix mois. Il est remarquable que les virulences des microbes en tubes fermés, comme il vient d'être dit, restent semblables à celles du début, tandis que les cultures témoins exposées à l'air sont mortes ou moins virulentes.

Le virus abandonné à l'air dans un bouillon, même très favorable à sa croissance, est en état de variation continue aboutissant tôt ou tard à la perte de la virulence et à la mort. Et le phénomène est de même, qu'il ait lieu dans le muscle de la poule résistante, où la nutrition suspendue 
affaiblit la respiration locale et par suite celle du microbe, ou dans le matras de culture largement aéré ; il n'y a que des différences de degrés qui traduisent la merveilleuse faculté d'acconmodation des êtres vivants.

Le microbe jeune, dans le milieu le plus favorable, est ténu, mais, lorsqu il vieillit, ses granules sont encore réduits et le liquide, trouble au début, s'éclaircit précisément parce que la taille de l'organisme diminue. Alors que l'inoculation du premier état aurait tué vingt poules sur vingt, pullulant au bout de vingt-quatre à quarante-huit heures dans tous les organes, le microbe vieilli par un séjour de quelques semaines dans son bouillon aéré déterminera une maladie réelle, caractérisée encore par une phase d'inappétence, d'abattement, de forme en boule, mais la maladie n'est pas mortelle. On constate, comme dans le cas précédent, durant cette phase, que le microbe répandu à partir du point d'inoculation est entraîné dans tous les tissus. Le malaise passe, les poules reprennent peu à peu toutes les apparences de la santé. Il y a eu choléra bénin n'entraînant pas l'issue fatale.

Or, désormais ce lot de poules, rendu malade expérimentalement, mais guéri parce que l'organisme a résisté à l'invasion du microbe atténué, ce lot de poules, dis-je, devient insensible à l'inoculation du microbe le plus virulent. Il est vacciné. La vaccination n'est qu'un corollaire, mais certes le plus important, de la résistance acquise par l'organisme, et PasteUr se consacre tout entier à son étude. Il note de suite ce fait capital que la localisation du Bacille ne limite pas la résistance aux parties voisines. Elle est complète pour tout le corps de l'animal ; l'inoculation ultérieure du Bacille très virulent est suivie, tant dans le muscle réparé que dans les muscles intacts, de lésions insignifiantes, 
rapidement localisées et réparées, le volume et le poids des séquestres étaient de beancoup inférieurs à ceux résultant de la première opération. La poule elle-même est à peine souffrante ; elle pourra ingérer le microbe par les voies digestives sans en être affectée, et, désormais, elle pourra vivre impunément au milieu des poules malades. L'immunité est acquise pour la durée de la vie de l'individu.

De nombreux essais ont montré que les lapins, les souris, succombent rapidement aux inoculations du choléra virulent et, d'après PaSTEUR, les lapins peuvent, comme les poules, succomber à des ingestions avec la nourriture. Les cobayes, les moutons, résistent, et l'inoculation expérimentale n'est généralement suivie que de lésions locales, d'abcès où le microscope permet de découvrir des colonies de Bacilles. Inoculant le pus à des poules et à des lapins, la maladie est presque toujours grave et fatale, tandis que le cobaye résiste, à moins que l'inoculation ne soit faite dans les cavités des séreuses péritonéales, qui, elle, est mortelle. On conçoit que le cobaye puisse héberger pendant des semaines et des mois le parasite sans dommage apparent et provoquer des épidémies fatales dans les basses-cours.

Plus tard, Cornil et Touper (1888) ont décrit une Bactérie ovoïde de un millième de millimètre de long sur un demi de large, ayant déterminé une épidémie chez les canards du Jardin d'acclimatation de Paris ; les cultures en bouillon ont un aspect différent de celles du Choléra étudié par Pasteur, et d'ailleurs les poules et les pigeons ne succombent pas aux inoculations, qui sont rapidement fatales aux canards ; les poules et les pigeons qui ont résisté au Choléra du canard ne sont pas vaccinés pour le Choléra des poules. C'est done un tout autre organisme. 
En 1891, LUCET décrivit une troisième affection des poules et des dindes, due elle aussi à un Bacille de dimensions analogues ; KLeIN (1889) l'avait sans doute déjà observé en notant le principal caractère différentiel, qui est l'absence de l'état somnolent des poules malades.

Voilà bien des analogies; les réactions des individus atteints sont presque identiques et les accidents rapides. Pour distinguer ces espèces ou ces formes de maladies, il fallait, d'une part, la sécurité des méthodes de culture pures si perfectionnés par PASTEUR, et, d'autre part, la connaissance exacte des modifications possibles par la culture in vitro sur des milieux définis et variés. Ainsi fut conservée intacte la notion de spécificité, qui aurait pu être largement ébranlée par la seule découverte de l'atténuation. 


\section{LUTTE POUR LA VIE SÉLECTION ET CARACTĖRES ACQUIS}

Vers 1860 , on désignait sous le nom de maladies charbonneuses une foule d'affections différentes par leur nature, par leur siège, par leur gravité, ayant pour caractère commun l'altération du sang. L'une des formes, qui a été l'objet d'études suivies et d'expériences remarquables par PAsteur, Joubert, Cimarmerland et MI. E. Roux, attaque les chevaux, les pores, davantage les boufs et surtont les moutons. Sans avoir des caractères franchement épidémiques, elle persistait dans certaines régions telles que l'Eure-et-Loir et y déterminait des pertes très lourdes. Certains champs bien connus des éleveurs, les champs maudits, étaient abandonnés en raison de la fréquence des accidents qui suivaient leur paturage par les moutons.

Inspiré par la lecture des recherches de Pasteur sur le vibrion de la fermentation butyrique, Casimir Daraine, biologiste réputé par l'étude des parasites entozoaires des animaux domestiques et de l'Anguillule des Blés, signala, en 1863, la présence d'un organisme inférieur, d'un infusoire comme il l'avait d'abord appelé, allongé, immobile, qu'il avait aperçu dès 1850 dans le sang des animaux succombant à la maladie connue sous le nom de sang de rate. Dans plusieturs notes à l'Académie des Sciences et à la Société de Biologie, il établit la présence constante dans le 
sang de ce qu iil appelle à partir de cette date Bactéridie et, «n. 1864, il la déconvre dans la pustule maligne chez l'Homme. Comme Pasteur, Daraine était fort réservé dans ses affirmations, et il fallut l'intervention de deux contradicteurs, Lfplat et JaILlard, qui annonçaient n'avoir pas trouvé la Bactéridie chez des vaches mortes du charbon inoeulé, pour provoquer de nouvelles études. Aualysant les symptômes décrits par ses contradictenrs et les conditions de l'inoculation, Davaine reconnut que ce n'était pas réellement d'une maladie charbonneuse qu'avaient été atteints les animaux examinés et il réussit à séparer (1865), par des caractères définis, le vrai charbon de la septicémie expérimentale.

Le caractère distinctif des Bactéridies du charbon était de se former pendant la vie de l'animal malade et de disparaître par la putréfaction peu de temps après la mort. Le sang frais contenant des Bactéridies et inoculé anx petits Mammifères, lapins, cobayes, souris, déterminait la maladie charbonneuse; mais il perdait cette propriété dès que la putréfaction faisait disparaître les Bactéridies. La pustule maligne de l'Homme n'était qu'une variété de la maladie charbonneuse, car elle renfermait la Bactéridie et produisait par inoculation le sang de rate chez les animaux. Davaine fit plus; il situa la Bactéridie dans son groupe naturel, parmi les végétaux, dans le voisinage des Conferves, et nota ses changements de forme avec le milieu, avec l'hôte. Il contribua avec PASTEUr à perfectionner la technique des inoculations et des dosages des Bactéridies. Il est impossible de ne pas associer le nom de Davaine à la série des améliorations techniques que PASTEUR et ses collaborateurs apportèrent longtemps après cette date dans la bactériologie expérimentale. Dès 1868, Davaine publie les Expériences rela- 
tives à la durée de l'incubation des maladies charbonneuses et à la quantité de virus nécessaire à la transmission de la maladie, présentées à l'Académie de Médecine, et, en 1869, les Expériences ayant pour but de prouver que les Bactéridies constituent seules le virus charbonneux. Davaine dilua du sang charbonneux avec une grande quantité d'eau, le laissa déposer dans une haute éprouvette et étudia la Bactéridie abondante au fond. Prélevant avec une pipette du liquide à des hauteurs différentes, il constata que les couches inférieures seules, renfermant les Bactéridies visibles, donnaient le charbon. Il suffit d'un millionième de goutte de sang infecté par les Bactéridies pour tuer un cobaye, et la durée d'incubation, e'est-à-dire l'intervalle de temps qui s'écoule entre le moment de l'inoculation et celui de l'apparition des phénomènes morbides, est en rapport avec la quantité de virus inoculé. Davaine prouva encore que toujours, lorsqu'il y a charbon typique, la Bactéridie est présente ; le sang de l'animal qui va mourir n'est contagieux que lorsqu'on y trouve la Bactéridie ; jusque-là ses effets sont nuls. Quelques jours après la mort, lorsque la putréfaction commence, le sang ne renferme plus trace de Bactéridie; son inoculation peut produire une maladie, comme l'avaient vu LePLAT et JAILLARD, mais cette maladie n'est pas le charbon.

Le filtrat du sang d'un animal charbonneux sur un manchon de terre poreuse a laissé sur celle-ci toutes ses Bactéridies ; très virulent avant l'opération, il est devenu inoffensif. Davaine fit mieux ; il montra le rôle des filtres naturels dans la propagation des maladies de la mère à l'enfant. Voici une brebis à terme ; elle meurt du charbon ; son sang prélevé immédiatement renferme la Bactéridie et rend les inoculations funestes ; au même moment, prélevez le sang dans 
le corps du fœtus avec certaines précautions pour ne pas y introduire le sang maternel, il ne renferme pas la Bactéridie et son inoculation ne communique pas le charbon.

Il semble qu'après ces démonstrations la preuve de la relation nécessaire et suffisante entre la Bactéridie et le charbon devait être définitive. En fait, les objections naissent et des années s'écoulent sans que la conviction soit établie. Heureusement, d'ailleurs, car l'intervention de PASTEUR n'étant pas nécessaire, ce dernier n'aurait peut-être pas eu l'occasion de découvrir l'atténuation des virus.

Dans la suite (1876), Косн imagine un traitement qui fait sporuler la Bactéridie, dénommée alors, après une étude de F. CoHn, Bacillus anthracis. Lorsqu'on place dans du sérum les bacilles du sang de rate, en les maintenant au contact de l'air humide et à une température de 35 à 37 degrés, ils s'allongent considérablement, donnent naissance à des filaments dix fois, cent fois plus longs ; leur contenu perd sa transparence et bientôt se remplit de granulation réfringentes, très rapprochées, régulièrement espacées, qui se séparent enfin et constituent de véritables spores internes on çorpuscules, comme F. CoHn en avait découvert récemment dans plusieurs autres espèces de bactéries. Il y a une température optima pour la sporulation ; vers 35 degrés, les spores apparaissent en moins de vingt-quatre heures; à 18 degrés, elles n'apparaissent qu'au second ou au troisième jour ; le développement du bacille s'arrête au-dessous de 12 degrés et au-dessus de 45 degrés. Aru cours de ces opéra. tions, la présence d'air est indispensable ; si on le supprime, en quelques jours le Bacille meurt sans sporuler, par désorganisation rapide. Les spores du Bacillus anthracis, mises dans du sérum, germent et reproduisent des bacilles; ino- 
culées à des animaux sains, elles déterminent la mort avec tous les symptômes du sang de rate. Mises à l'abri de l'air, dans les conditions qui détruisent les bacilles, elles avaient conservé toute leur activité après quatre années.

Pasteur et Joubert, en 1877 (1), exposent quelques faits nouveaux relatifs à la formation des spores de la Bactéridie: "Dans l'animal charbonneux, au moment de la mort, la Bactéridie est exclusivement formée de filaments articulés sans le moindre corpuscule-gerne. Au contraire, une eulture dans l'urine donne, après un repos de quelques jours, une grande abondance de corpuscules brillants associés ou non à des Bactéridies filiformes. Si l'on précipite par l'alcool le sang charbonucux et qu'on fasse dessécher rapidement le précipité qui enferme dans ses mailles toutes les Bactéridies, celles-ci, sans exception, devienuent absolument inertes. La même opération, appliquée aux corpuscules-germes de la Bactéridie, conserve à ces derniers leur forme, leur aspect et leur puissance d'inoculation ultérieure ou leur faculté de développement dans l'urine neutre. On démontre ainsi qu'ils n'ont rien perdu de leur vitalité propre et de leur terrible action sur l'économie. ") Répétant l'expérience de PAUL BERT, qui avait tué les Bactéridies par l'action de l'oxygène à haute pression, PASTEur et Joubert constatent que les corpuscules-germes ont résisté pendant vingt et un jours à dix atmosphères d'oxygène pur sans perdre leur faculté de reproduction.

I a Bactéridie se comporte vis-à-vis de l'oxygène de l'air à l'opposé des ferments ; elle n'est pas capable d'en emprun-

1. - Charbon et septicémie, communication à l'Académie de Médecine, 17 juillet 1877. 
ter anx corps complexes qui l'entourent; elle a besoin d'oxygène libre; elle se résout en fines granulations amorphes dès que l'oxygène a disparu. Or, la vie empêche la vie : " Le sang vivant, en pleine circulation, est rempli d ime multitude infinie de globules qui ont besoin, pour vivre et pour accomplir leur fonction physiologique, de gaz oxygène libre; on peut dire que les globules du sang sont des êtres aérobies par excellence. Lors done que la bactéridie charbonneuse pénètre dans un sang normal, elle y rencontre un nombre immense d'individualités organiques prêtes à ce qu'on appelle quelquefois, dans un langage imagé, la lutte pour la vie, en d'autres termes à s'emparer pour ellesmêmes de l'oxygène nécessaire à l'existence des bactéries. "

Voilà, pour nos savants, l'explication de la résistance des Oiseaux au Charbon. Inoculée dans lenr orgauisme, la Bac. téridie ne se multiplie pas ; en peu de temps, le microscope est incapable d'y déceler les bâtonnets; dans le sang de poule hors de l'organisme, et mieux encore dans son sérum, la Bactéridie se multiplie rapidement. Le cochon d'Inde est à peine affecté par l'inoeulation de la Bactéridie ; l'injection dans la veine jugulaire ne provoque pas plus de désordres que l'injection sous-cutanée ; ensemencée hors du corps, dans le sang du même animal, en quelques heures elle y pullule.

L'urine neutre ou légèrement alcaline est num des meilleurs milieux de culture pour la Bactéridie; ensemencée seule, elle y forme, dans l'intervalle de quelques heures, nu feutrage de filaments cotonneux. Mais ensemencée avec des bactéries communes, avides d'oxygène elles aussi, la bactéridie charbonneuse ne se développe pas, ou très peu! ; rapidement elle succombe dans la lutte pour l'oxygène. "On arrive à ce résultat surprenant qu'on peut introdnire à pro- 
fusion dans un animal la bactéridie charbonneuse sans que celui-ci contracte le charbon; il suffit qu'au liquide contenant en suspension la bactéridie, on ait associé en même temps des bactéries communes. ")

Ce sont les plus remarquables exemples qu'on puisse donner de la concurrence vitale ; PASTEur et Jovibert les signalent pour expliquer les contradictions apparentes entre les épreuves de Davaine et celles de Juillard et Leplat, auxquelles d'ailleurs Davaine avait parfaitement répondu. Nous voyons maintenant pourquoi la septicémie, maladie due à divers vibrions putrides anaérobies, a pu, si souvent, être confondue avec le charbon.

$$
\text { *** }
$$

PASTEUR en déduit, un an plus tard, que la propagation du charbon dans les champs maudits résulte de la fréquence à la surface du sol de corpuscules-germes qu'il y prélève, qu'il cultive dans les milieux les plus favorables, et qui communiquent le charbon-type caractérisé par la Bactéridie. Un membre très influent de 1'Académie de Médecine, CouIs, vétérinaire distingué, oppose à ses conclusions des négations réitérées ; PASTEUR, avec une fougue qui l'ont rendu terrible pour ses contradicteurs, le poursuit dans ses argumentations. Après avoir prétendu que les ganglions des animaux qui allaient mourir du charbon renfermaient le principe toxique cause du mal avant l'apparition de bactéridies dans le sang, Coun affirme que la maladie est introduite, à côté de la bactéridie inoffensive, par la petite quantité de sang en déeomposition qui l'accompagne au cours des prélèvements. BouLEY, secrétaire général de la Société centrale de Médecine vétérinaire, collègne de PAsteur à 
l'Académie des Sciences, lui expose les vues de CoLin avec quelques observations. Je ne puis résister à la tentation de citer la réponse de PaSTEUR parce qu'elle est, à la fois, un modèle d'exposition des faits dans une polémique et une démonstration de la sécurité des preuves réunies par PASTEUR avant toute publication :

Arbois, le 18 août 1877.

\section{Mon cher confrère,}

Je m'empresse de vous répondre.

Oh ! que j'aurais envie de prendre à la lettre l'honneur que vous me faites en in'appelant " votre maitre " et de vous donner une bonne et verte leçon, homme de peu de foi, qui paraissez avoir été touché par la lecture de M. Colin à l'Académie de Médecine, puisque vous dissertez encore sur la possibilité d'un agent virulent et que vos incertitudes semblent calmées par une Note nouvelle (de M. Toussaint), présentée par vous lundi dernier à l'Académie des Sciences.

Laissez-moi vous dire en toute franchise que vous n'êtes pas assez pénétré des enseignements que reuferment les lectures que j'ai faites, en mon nom et au nom de M. Joubert, à l'Académie des Sciences et à l'Académie de Médecine. Croyez_vous donc que je vous les aurais faites, ces lectures, si elles avaient eu besoin des confirmations dont vous me parlez, ou si les contradictions de M. Colis avaient pu les atteindre ? Vous savez bien quelle est ma situation dans ces graves controverses; vous savez bien qu'ignorant comme je le suis de toutes les connaissances médicales et vétérinaires, je serais immédiatement taxé de présomption si j'avais la témérité de prendre la parole sans être armé pour le combat, la lutte et la victoire. Tous à l'envi, et avec raison, vétérinaires et médecins, vous me jetteriez la pierre si j'apportais dans vos débats des semblants de preuves.

Comment n'avez-vous pas remarqué que M. Colın a travesti, je devrais même dire supprimé, parce qu'elle gênait ses croyances, l'importante expérience des cultures successives de la bactéridie dans l'urine ? Mêler une goutte de sang charbonneux à de l'eau, à du sang pur, à du sérum, ou à l'humeur de l'œil, comme l'ont fait Davaine, Koch et M. Colin lui-même, puis inoculer une partie du mélange et provoquer la mort, c'est laisser le doute dans l'esprit sur la cause de la virulence, principalement depuis les célèbres expériences de Davalne sur la septicémie. Tout autre est notre expérience. La bactéridie est cultivée une première fois en prenant pour semence une trace microscopique de sang char- 
bonneux, puis on passe à une seconde cuiture, à une troisième..., à une dixième..., à une centième, en prenant toujours pour semence d'une culture une gouttelette de la culture précédente. Si vous supposez que la semence pour chaque culture est délayée dans cent fois son volume, ce qui est encore infiniment au-dicssous de la vérité, la goutte de sang charbonneux de l'origine se trouvera à la fin diluée dans un nombre de gouttes d'urine représenté par le nombre 100 élevé à la centième puissance, e'est-à-dire par l'unité suivie de 200 zéros. Ce serait une absurdité d'adnettre que la dernière virulence emprunte son pouvoir, non à la bactéridie qui s'est multipliée dans chaque culture, mais à un agent virulent existant dans la goutte de sang originaire. N'êtes_vous pas satisfait? Faites alors mille cultures successives au lieu de cent. Voilà notre expérience. Après l'avoir rendue ridiculement, M. Colin ne craint pas de dire "que M. Pasteur se sert d'une dilution beaucoup plus concentrée que les dilutions de Davalne ".

M. Colin objecte ensuite que l'expérience de la filtration sur le plâtre peut altérer les ferments, les diastases, les agents virulents solubles. Comment M. Colın ne voit-il pas qu'une telle oljjection n'a de valeur que par la preuve qu'il en pourrait donner? Or, quoi de plus simple que de délayer du sang charbonneux avec dn plâtre et de l'eau, et d'inoculer ensuite une portion de ce mélange ? En vingt-quatre heures, $M$. Colın aurait su à quoi s"en tenir; mais non : M. Colın croit à la présence d'une matière soluble virulente, parce qu'il reut que cela soit ainsi. Il y a dix ou donze ans, nous apprend_il, qu'il étudie la maladie charbonneuse et il en est à la cinq-eentième expérience. Aucune de ces cinq cents expériences u'ayant été significative sur le point en litige, il n'admet pas qu'on puisse en faire ayant ce caractère. Celles qui ont ce caractère sont ponr lui non arenues; s'il en parle, c'est ponr les travestir ou '́lever contre elles des subtilités de dialectique.

Si la filtration sur le plâtre lui laissait des doutes, pourquoi ı'a-t-il rien dit de celle des caves de l'Observatoire ? Ici, la filtration, étant faite par simple décantation, devait le satisfaire pleinement.

Bref, la lecture de la communication de M. Colin in'a laissé cette inmpression qu'il est en proie à ce travers d'esprit, plus fréquent qu'on ne se l'imagine, et que caractérise l'épitaphe de mes Etudes sur la bière, empruntée à l'un de nos plus grands écrivains: "Le plus grand déréglement de l'esprit est de croire les choses parce qu'on veut qu'elles soient. " M. CoLis croit à un agent virulent placé clans le sang à côté des bactéridies, parce qu'il veut que cela soit ainsi; et ce qui le montre bien, c'est son refus obstiné de prendre en considération le véritable caractère des expériences qui démontrent invinciblement l'absence de tout agent virulent.

L'expérience de M. Toussaint sur la perte de virulence du sang charbonnenx à l'abri de l'air est l'une de nos expériences. Son 
procédé d'extraction du sang pur est inférieur à l'un ou à l'autre des nombreux dispositifs que j'emploie depuis longtemps.

L'assertion de la deuxième édition de Hurtrel D'Arboval par' ZunDel n'est qu'une assertion. Attachez-vous donc, dans ces études délicates, a distinguer l'assertion d'une preuve ? Oni, ZuNDEL assure que la bactéridie prive le sang de son oxygène, mais la conséquence qu'il en déduit n'est qu'une supposition.

Que la bactéridie absorbe l'oxygène, c'est une chose évidente, parce que tout organisme absorbe l'oxygène libre, qu'il soit aérobie ou anaérobie; mais, ce que nons avons prouvé, et ce que l'on n'avait pas fait auparavant, la bactéridie absorbe jusqu'aux dernières portions de l'oxygène. Voilà la preuve, sans laquelle on ne peut conclure rigoureusement à la nécessité de l'axphyxie.

Au moment de clore cette lettre, je reçois le numéro du 15 aoùt de votre excellent Redueil de Médecine Vétérinaire, et j'y lis le passage suivant, que je reproduis textuellement :

"Si tel était, en effet, le rôle de la bactéridie, dit M. Colıs, si elle était la condition nécessaire de la virulence, on devrait la retrouver dans le sang de l'animal inoculé, alors que ce sang inoculé lui-même est capable de transmettre le charbon... Or, si d'heure en heure on extrait du sang d'un animal inoculé et si, pour essayer sa virulence, on le fait servir chaque fois à de nou. velles inoculations, on constate qu'après quelques heures il commence à communiquer le charbon, bien qu'on n'y aperçoive pas encore de bactéridies. - La virulence sans bactéridic s'oloserve également dans le sérum obtenı après la coagulation du sang charbonneux. Les bactéridies étant retenues dans les mailles du coagulum, le sérum n'en présente point, mais il communique le charbon quand on l'inocule."

Je puis opposer à ces assertions de M. Coun une contradiction sans réserve. Nous avons fait de notre côté l'expérience que rapporte M. Colıs, mais avec une rigueur qu'il lui était impossihle d'appliquer parce qu'elle repose sur les faits nouveaux communiqués dans notre Note du 30 avril dernier. Jamais le sang qui ne contient pas de bactéridies ne communique le charbon. Ce qui a trompé M. Colin, e'est l'insuffisance du microscope pour accuser la présence ou l'absence des bactéridies, quand le sang n'en contient qu'un nombre infiniment restreint. Supposez une bactéridie seulement dans une goutte de sang d'un animal inoculé ; ce serait presque un miracle que de tomber, dans l'observation au inicroscope, sur ce petit filament. La recherche d'une nouvelle planète serait plus facile. Mais si vous semez cette même goutte de sang dans 10 on 20 centimètres cubes d'urine nentralisée et dans des conditions d'une pureté telle qu'aucun organisme nouveau ne 
puisse nuire au développement de l'unique bactéridie de la goutte, cette bactéridie se multipliera avec une aisance parfaite. Peu à peu ses générations successives rempliront le liquide, et cette fois, même sans l'aide du microscope, à l'œil nu, on constatera facilement la présence des bactéridies. Voilà ce qu'ignorait M. Colın. S'il eût pu recourir à ce genre d'épreuves, il aurait vu, comme nousmême, que si le sang communique le charbon, alors même que le microscope est infidèle à faire reconnaître la présence des bactéridies, l'expérience de l'ensemencement dont je viens de parler accuse l'existence de celles_ci. Il aurait observé, en outre, comme nous l'avons fait nous-même, que toutes les fois que l'urine reste stérile, le sang de l'animal inoculé ne communique jamais le charbon.

M. Colin dit encore qu'une fois il aurait rencontré un sang chargé de bactéridies qui ne pouvait communiquer le charbon. Cette expérience, que je considère comme tout à fait erronée, n'aurait de valeur que si M. Colin avait prouvé en même temps qu'il avait eu réellement affaire à des bactéridies. Je mettrais au défi l'observateur le plus habile d'affirmer que tel ou tel bâtonnet est une bactéridie charbonneuse par une simple observation microscopique. L'expérience dont il s'agit est donc non avenue.

Croyez aux sentiments affectueux de votre dévoué confrère.

L. Pasteur (1).

Le mois suivant, PASTEUR manifeste par une lettre insérée dans le même Recueil, à propos de la citation incomplète a'un texte rédigé dans les comptes rendus de l'Académie, d'une part, la préoccupation que tout contradicteur doit avoir de la citation exacte des textes, d'autre part, l'intérêt passionnant avec lequel il suivait les applications de ses principes dans la lutte contre les maladies infectieuses :

Arbois, ce 7 septembre 1877 .

C'est M. Papillon qui est responsable de l'expression hematocristalline. Pour moi, je ne l'ai jamais employée ni prononcée nulle part.

1. - Extrait du Recueil de Médecine Vétérinaire, 6e sér., t. IV, 1877, pp. 915_918. 
Tout ceci, je le répète, mérite à peine une mention; néanmoins, on $\mathrm{y}$ voit une fois de plus la preuve qu'il est préférable de rappeler les observations dans les termes mêmes dont se sont servis ceux qui les ont faites les premiers.

Je suis heureux également de trouver l'occasion de vous montrer que je cherche à m'instruire dans la médecine vétérinaire, puisque c'est en parcourant l'une de vos publications que j'ai rencontré le sujet de cette lettre. Si j'étais jeune, et même à mon âge, si j'étais plus valide, j'irais me constituer élève de l'Ecole d'Alfort. Les lectures des ouvrages vétérinaires me mettent la tête en feu.

Votre bien dévoué confrère,

\section{Pasteur (1).}

A cette même date, PASTEUR avait entrepris, nous l'avons vu, la rédaction définitive d'un ouvrage sur la Dissymétrie moléculaire. Convaincu qu'il tient les fils conducteurs de la méthode qui le conduiront à trouver le point faible du fléaru le Charbon, il n'hésite pas à changer de carrière, à abandonner ses cristaux et ses fermentations pour créer, en pionnier de la guerre aux maladies virulentes, une voie féconde en tant de mémorables découvertes. Nous avons vu le parti qu'il tira de l'étude du Choléra des poules; nous nous limiterons à l'examen de l'essentiel de ses découvertes à propos de l'étiologie du Charbon et de la préparation du vacein charbonneux.

En 1878, le ministre de l'Agriculture avait chargé, d'une part, M. Pasteur, assisté de son préparateur M. ChamberLAND et de M. VINSOT, élève sortant de l'Ecole d'Alfort, d'autre part, Mr. ToussaIn', professeur de physiologie à l'Ecole vétérinaire de Toulouse, de missions parallèles pour suivre le Charbon dans ses ravages en Beauce et préciser les causes des épidémies locales.

Pour Pasteur, le Charbon dit spontané devait nécessai-

1. - Même recueil, p. 922 . 
rement procéder de la Bactéridie charbonneuse, comme le Charbon expérimental. On put provoquer, par l'ingestion d'aliments arrosés de cultures bactéridiennes, des accidents foudroyants, identiques à ceux du Charbon spontané ; mais ils furent rares. En associant à la nourriture infectée tout ee qui pouvait provoquer des blessures dans les parties antérieures du tube digestif, dans la bouche, antour de la langue. dans le pharynx, la mortalité fut notablement accrue; des chardons secs mêlés à la luzerne, des barbes de l'épi de l'orge, entraînaient des déchirures dans la bouche ou l'arrière-gorge, facilitaient l'inoculation de la bactéridie. PASTEUR trouve une confirmation à ses inductions dans le fait bien connu que les grandes chaleurs et les sécheresses favorisent le développement et multiplient les ravages du Charbon. Pour diminuer les chances de l'inoculation, il faut purger les fourrages des plantes piquantes, supprimer les aliments très sees l'hiver comme l'été, les menues paille, par exemple ; ne faire manger celles-ci qu'après les avoir mouillées, et mieux, les avoir fait fermenter avec des fourrages verts. Enfin, éviter de donner des aliments chargés de la poussière siliceuse des routes.

$$
* *
$$

Vous lirez dans les ouvrages de Duclaux, dans le remarquable article de M. le docteur Roux sur l'oruvre médicale de Pasteur (1), comment il découvrit le rôle des Vers de terre qui, dans les “ champs maudits », ramènent à la surface

1. - Paru dans l'Agenda du Chimie, 1896, et reproduit dans la brochure éditée par HacheTte à l'occasion du centième anniversaire de la naissance de Pasteur. 
du sol les spores du C'harbon cléveloppées dans le sang aéré (urine sanguinolente, épanehements nasaux) des animaux morts du sang de rate et enfouis à plusieurs mètres ; vous lirez, racontée par un témoin et opérateur, la célèbre expérience de Pouilly-le-Fort, dont M. le docteur Rocx dit: "Comme nous remarquions que le programme était sévère, mais qu'il $n$ 'y arait plus qu'à l'accomplir puisqu'il était signé, Pasteur ajouta : "Ce qui a réussi sur quatorze mou" tons au laboratoire réussira aussi bien sur einquante à "Melun."

Ces leetures, nécessaires, et ies lettres que je viens de reproduire, donnent l'idée de l'atmosphère de fièvre, d'audace de pensée et de prudenee, indispensables dans les actes de ceux qui ont la eertitude de faire de grandes ehoses, et PASTEUR, à eette date, avait la eertitude. Les publications dont nous tirons nos documents ne sont que des résultats condensés de nombreuses suites concordantes d'épreuves répétées, examinées sous toutes leurs faces. En moins de cinq années, Pasteur a déeouvert :

La raison probable de la résistance naturelle au Charbon des races de moutons algériens, fait eité et vérifié par Chauveau ;

Les conditions particulières qui peurent rendre réceptif un animal, la poule par exemple, normalement réfractaire au Charbon ;

Les procédés préeis d'atténuation graduée de la baetériaie la plus virulente ;

Ia réaction parallèle à tous les degrés des individus réceptifs, ee qui permet de graduer la maladie à volonté ;

La vaceination en une ou plusieurs inoculations ;

Le fait qu'en ce cas (contrairement au Choléra des poules) la résistanee n'est pas acquise par une toxine libre 
dans le milieu inoculé, mais bien par une réaction intime du patient ;

Le retour possible à la virulence, en passant par tous les degrés, de l'état presque inactif à l'état le plus dangereux ;

La stabilité du virus totalement inactif qui, lui, ne peut faire retour, dans le temps consacré aux épreuves, à la forme virulente.

Il y a dans toutes ces découvertes de principe assez de matière pour une nouvelle série de Conférences; surtout dans les détails de la technique, il y a des aperçus si suggestifs, qu'il faut lire dans les comptes rendus de l'Académie des Sciences et de l'Académie de Médecine, dans les Sociétés savantes de Biologie, de Médecine vétérinaire où, désormais, toutes les séances renferment des exposés des études de Pasteur lui-même, et plus encore des découvertes des élèves inspirés par lui. Les techniques de la préparation et de l'utilisation des vaccins se perfectionnent ou s'adaptent aux circonstances dans les études sur la septicémie, sur la fièvre puerpérale, sur le rouget des pores, enfin sur la Rage.

La circonstance la plus minime peut entraver la marche normale d'une fermentation ; de même une très légère modification du milieu interne, ici le sang des animaux, entraîne des résultats très différents quant à la résistance du patient.

Le mouton français succombe à l'inoculation du Charbon normal que le Ver de terre vient de ramener à l'état de spore à la surface du " champ maudit ». Il résiste à un Charbon atténué, mais est gravement malade ; après quoi, le voilà vacciné contre le Charbon, mortel pour un animal frais. Le mouton algérien est réfractaire et ne meurt généralement pas de l'inoculation d'un virus mortel pour le mouton français ; mais en exaltant la virulence de ce dernier, ou 
en affaiblissant le mouton algérien, le microbe accomplira son cuvre destructrice.

Pasteur n'y voit qu'une lutte pour l'oxygène dans l'intérieur du sang des divers animaux; les globules rouges, croit-il, disputent pas à pas la route à la bactéridie ; l'atténuation ou la diminution d'activité de la bactéridie perniet aux globules rouges de l'emporter; ils l'emportent à coup sûr s'ils ont déjà été préparés à la lutte par la rencontre avec une bactéridie plus faible et de même espèce.

Pasteur en donne comme preuve que la poule, d'ordinaire réfractaire au Charbon, meurt à la suite d'inoculations qui suivent de près un bain forcé qui refroidit l'animal. Il montre, d'autre part, que le terrain peut être aménagé de façon à accentuer la résistance. Ayant reconnu que la Bactéridie charbonneuse se développe péniblement dans un liquide de culture du Bacille du Charbon des poules, il prévoit que les poules vaccinées contre le Choléra doivent être plus résistantes à l'invasion de la bactéridie, et l'expérience justifie cette présomption.

Depuis, E. Мeтchnikoff, M. L. Bordet, nous ont amené à d'autres conceptions ; mais il me suffit de suivre Pasteur dans ses déductions. Presque toujours elles l'ont conduit à la solution rapide et définitive.

Le vieillissement de la bactéridie est difficile parce qu'elle ne tarde pas à sporuler, et alors ne vieillit plus. PASTEUr, Chamberland et Roux constatent que l'action prolongée durant un mois d'une température-limite sans permettre la sporulation, tue graduellement la bactéridie. Après huit jours, elle devient inoffensive pour le cobaye, le lapin, le mouton; elle provoque une fièvre bénigne qui vaccine le patient. En renouvelant les oultures dans cet intervalle par des passages sur les animaux sensibles, on remonte la pente 
et on retrouve la virulence première. Chaque état, cultivé en milieu stérile, défini, conserve indéfiniment sa virulence propre.

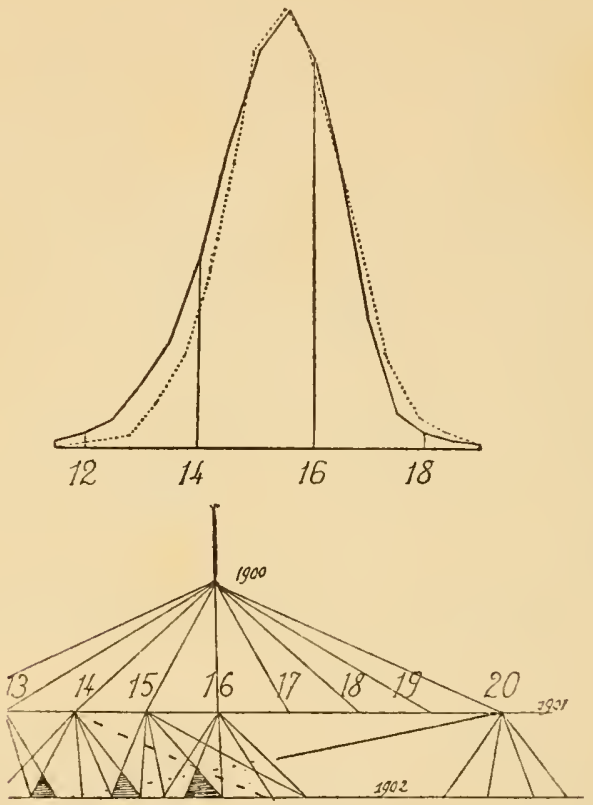

Fig. 50. - Sélection de la Betterave à sucre, à Naarden (Hollande); en haut, Polygones de variations de la teneur en sucre de 40.000 tubercules (d'après DE VRIES); en bas, modes de ségrégation el des retours des descendances successives 1900 , 1901,1902 , pour les teneurs diverses en sucre $14,15,16, \ldots 20$; les retours sont très étalés pour cette dernière teneur.

La sélection de la Betterave à sucre nous offre des étapes analogues, chacune d'elles étant réversible. A Naarden, en Hollande, d'après $\mathrm{H}$. DE VRIES, on analyse chaque année 40.000 tubercules pour maintenir la moyenne de la teneur en sucre à $16 \%$. Ce qui ne veut pas dire que tous les tuber- 
cules donnent re taux; ils en donnent de plus bas et aussi de plus élevés; rapidement, on peut descendre à une moyenne 15, 12 et même inférieure, en choisissant les progéniteurs dans les lots correspondants. La sélection agit comme le montre la figure ci-contre ; au lieu de prendre l'ensemble de la récolte représentée par le polygone de variation en forme de cloche, on étale les tranches de variation au même degré et on ne prend qu'une tranche, celle à 20 par exemple, pour que, la régression agissant avec une amplitude connue pour la race, la moyenne de la descendance de l'année suivante soit précisément 16 . On réduit le nombre des examens, et, par' suite, celui des tubercules porte-semence si l'on adopte la méthode GoraIN, qui consiste à greffer sur différents tubercules ordinaires les dix à vingt bourgeons, ou yeux, qu'on sait détacher du collet des trabereules choisis. Ainsi la multiplication des individus se confond ici avec la multiplication des parties d'un' même individu, exemple précis de la définition que nous avons donnée plus haut de la lignée pure.

Restent les cas limites. Le principe de l'atténuation de la Bactéridie charbonneuse est, au fond, celui de l'atténuation du Bacille du Choléra des poules ; mais, alors rue le dernier ne donne jamais de spores, le premier en donne constamment si l'on reste an-dessous de 40 degrés et l'atténuation n'a pas lieu. "A la température de $42^{\circ} 5-43^{\circ}$, dit MI. le $\mathrm{D}^{\mathrm{r}}$ Roux (1), sous l'action de l'oxygène, l'atténuation se fait peu à peu, de sorte que l'on obtient une série de virus d'activité décroissante en puisant à divers moments dans la

1. - Docteur Roux : L'CEuvre médicale de Pasteur, Agenda du Chimiste, 1896. 
culture origine à $42^{\circ} 5$. Ces bactéridies atténuées conservent leur virulence affaiblie dans les générations successives développées à $30-40^{\circ}$; et, à cette température, elles donnent de nouveau des spores qui fixent cette virulence. On a done ainsi toute une gamme de virus de plus en plus faibles, que l'on peut reproduire à volonté. » Et un peu plus loin : "La virulenee est rune qualité que les mierobes peuvent perdre, ils peuvent aussi l'acquérir... Cultivée dans le eorps d'une souris âgée d'un jour seulement, la bactéridie (atténuée au point de ne plus tuer aucun animal adulte) reprend de son aptitude parasitaire. Avee le sang de eette souris, inoeulonsen une autre un peu plus âgée : elle périra. En passant ainsi des souris plus jeunes à des souris plus vieilles, nous arrivons à tuer les souris adultes, les cobayes, puis les lapins, puis les moutons, ete.....

“ Dans ees passages, la virulence est allée en augmentant. Cet aceroissement de virulence, que nous réalisons expérimentalement, se produit sans doute dans la nature, et nous eoncevons très bien qu'un mierobe d'ordinaire inoffensif pour une espèce animale puisse devenir meurtrier pour elle. Ne serait-ce pas ainsi qu'à travers les âges ont apparu les maladies infectieuses?"

Au cours de ses études sur l'atténuation de la Bactéridie charbonneuse, PASTEUR a obtenu une race asporogène, incapable désormais de fournir les spores qui, dans le cycle s.aturel de la maladie du sang de rate, sont l'élément essentiel de la propagation de la maladie. Chamberland et Roux montrent que la présence dans les bouillons de culture de traces d'antiseptiques $\left(1 / 600^{\mathrm{e}}\right.$ d'aeide phénique, $1 / 2000$ e de bichromate de potasse) donne le même résultat.

La perte de la faeulté de sporuler est done bien une réaction propre à l'espèce Bactéridie charbonneuse, réaction 
mise en aetivité par l'individu soumis à des eonditions défeetueuses de maturation. Nos variétés de Chrysanthèmes à fleurs pleines, les Topinambours qui ne se maltiplient que par tubercules, la plupart des arbres fruitiers à pépins ou à noyaux avortés (Poiriers, Kakis du Japon), ne sont autre ehose que des fragments d'individus propagés par boutures ou par greffe, dans eertains milieux, sans fournir désormais les éléments sexuels eapables de les régénérer. Le Bananier (Musa sapientium) en est un bel exemple; la séleetion de ces fruits sueeulents a fait négliger la conservation des individus à graines, et les formes existant aetuellement sont exclusivement parthénocarpes, sauf une forme instable des rochers de l'île Tahiti ; leurs fruits se développent sans la moindre formation d'embryons. Tôt ou tard, ees individus atteindront la vieillesse fatale et disparaîtront; la eulture soignée, la greffe sur des sujets choisis, les sauvent actuellement de la déchéance en raison des avantages matériels qu'ils proeurent à l'homme.

La Canne à suere fournit run bel exemple de cette dégénéreseence. Entre 1860 et 1900, les plantations de cette Graminée furent très gravement atteintes d'une maladie mierobienne se traduisant par l'extension de traînées rouges dans le cour de la Caune et connue vulgairement sous le nom de Sereh ; la variété Cheribon, l'une des plus appréciées à Java, en souffrit gravement. En l'étudiant, WAKkER s'apereut (1894) que toutes les Cannes en eulture de cette variété étaient strietement femelles. Pour la régénérer, il fallut introduire des variétés à pollen fertile.

Or, ees variétés sont très rares ; avant 1885, il n'y avait pas de graines connues ; en 1888, HARRISON et BowELL aux Barbades, en 1887, Solwedel, à Java, avaient signalé quelques levées de plantes, et l'on put, grâce à ces observations 
préliminaires, trouver des lots de Camnes dont le pollen était encore fécond.

La disparition totale des pollens aurait sans ancum donte entraîné la disparition rapide de l'une des plantes les plus utiles à l'homme. Le Sereh affecte les Cannes donnant $19 \%$ de sucre en moyenne. Par la pollinisation et le croisement, les races régénérées fournissent des plants domnant de 11 à $16 \%$ de sucre, état de sélection peu accusé, qui offre au Sereh une résistance totale.

Peut-on dire que la Canne Cheribon, incapable de fournir des graines, est une nouvelle espèce, une mutation de l'ancêtre, digne d'être classée à part ? N'y a-t-il pas plutôt dans cet exemple la preuve de la dégénérescence qui atteindra tôt ou tard le Charbon asporogène ?

Les races asporogènes de Champignons, de microbes, ne prouvent rien, ni pour ni contre la mutation spécifique. PASTEUR n'a jamais pensé qu'il avait obtenu par ces procédés de nouvelles espèces au sens propre du mot; sa doctrine de la spécificité, soutenue par les plus brillants de ses collaborateurs, n'a subi actuellement aucune atteinte. 


\section{RÉSUMÉ ET CONCLUSIONS}

Louts Pasteur étudia la série des tartrates au point de vue de leurs formes cristallines. Il constate que les sels dérivés de l'acide tartrique ordinaire extrait du tartre des vins présentent tous l'hémiédrie à droite et que leurs solutions dévient à droite le plan de polarisation de la lumière. Il sait, d'autre part, qu'un indnstriel d'Alsace a obtenu, il y a vingt ans, par hasard, une masse considérable d'acide racémique, corps ayant mêmes propriétés et composition que l'acide tartrique, qui en diffère seulement par l'inactivité des solutions de ses sels. Etonné du fait que les faces hémiédriques existent sur les cristaux des racémates de soude et d'ammoniaque, il dérouvre, dans tous les échantillons, des masses égales de cristaux à hémiédrie droite et de cristaux à hémiédrie ganche ; il les sépare sous le microscope, étudie leurs solutions à part et vérifie ainsi la règle prévue : l'hémiédrie à droite est liée à la polarisation à droite, l'hémiédrie à gauche à la polarisation à ganche; des mélanges à poids égaux des deux sous-espèces de tartrates donnent des solutions à pouvoir rotatoire nul, parce que les effets inverses et égaux se compensent.

Ce faisant, il opère comme le botaniste Jordan, qui distingue dans les espèces linnéennes des sous-espèces ronstantes et bien délimitées; il raisonne comme BERNARD DE Jussieu, inventant la subordination des caractères, lorsqu'il suggère que la concordance des formes hémiédres et de la polarisation dans le même sens traduit une propriété uni- 
que de la molécule de l'acide tartrique, sa dissymétrie. Désormais il adoptera, comme plus générale et plus exacte, la déviation de la lumière polarisée par les solutions comme définition de la dissymétrie moléculaire.

Il n'y a pas toujours concordance entre la forme des cristaux et la polarisation rotatoire. Par différentes épreuves, telles que la cristallisation dans des milieux divers, ou en présence d'impuretés, ou même par des essais de régénération, Pasteur montre que l'hémiédrie parfois latente ne se traduit qu'au cours de la cristallisation, qu'elle se présente lorsque quelque circonstance ralentit le dépôt. En un mot, il étudie les modifications de formes avec le milieu ambiant, avec l'âge et les phases de croissance, exactement comme les biologistes étudient les adaptations doubles, les variations saisonnières, les formes ataviques on régressives.

Dans un travail antérieur. il a mis en lumière que les substances dimorphes eristallisent avec des formes-limites très voisines les unes des autres, quoique classées dans des systèmes différents; elles présentent une forme stable et des états instables exactement comme les espèces critiques des botanistes, dont les variations à fleurs doubles, à métamorphoses sexuelles, sont bien connues parce que l'homme les recherche pour l'ornementation des jardins. Et même, lorsque les substances sont à la fois dimorphes et isomorphes, telles les Chlorures de Naphtaline et de Naphtaline monochlorée, la cristallisation, selon le hasard des circonstances, se produit en toutes proportions dans l'un ou l'artre système, fait très rare chez les végétaux, dont les Chimères de Morelle et de Tomate donnent l'expression correcte.

L'addition de bases minérales inactives à l'acide tartrique ne change rien à l'édifice moléculaire ; leur substitution se 
fait selon des poids définis et les composés sont isomorphes. Toujours, pour les simples mélanges de sels isomorphes, le pouvoir rotatoire est la moyenne arithmétique des pouvoirs rotatoires des composants ; souvent, pour les sels doubles, l'une des bases donne aux solutions son propre équilibre et il est possible de dire que certaines bases sont dominantes, d'autres récessives. En fait, nous trouvons dans ces substitutions qui n'altèrent pas la charpente moléculaire tous les traits de l'hérédité alternante, régie par les lois numériques de Mendel et relative à la transmission de particularités accessoires, indépendantes des caractères propres à l'espèce. Les modifications étudiées jusqu'à présent n'altèrent pas la dissymétrie ; l'essence spécifique est intacte.

Naudin a prouvé (1863) que l hybridation chez les végétaux aboutit d'ordinaire à la construction d'êtres mosaïques, ajustant côte à côte les éléments propres aux deux espèces affines, à peine fusionnés et tendant sans cesse à se dégager les uns des autres. Pasteur avait décrit dix ans plus tôt des mosaïques cristallines de bimalate d'ammoniaque et de bitartrate d'ammoniaque, qui sont l'expression régularisée, simplifiée, des mosaïques hybrides vivantes. Il a insisté souvent sur le parallélisme des propriétés de l'acide malique et de l'acide tartrique qui établit leurs affinités moléculaires; l'hybridation n'a lieu qu'entre espèces voisines.

Les mosaïques ne se produisent que par l'union de corps actifs, ayant chacun leur individualité. Le plus souvent, les affinités sont trop lointaines pour l'emporter sur les forces extérieures; alors l'ajustement des forces moléculaires est instable, irrégulier, et dépend si l'on veut des circonstances qui ont présidé à leur répartition. Les tartrates des bases organiques actives se comportent done comme les hybrides 
du Blé et du Seigle, comme les hỵbides des Tabacs. Ici, plus de règles, mais jeu des affinités propres à chaque groupe particulier, variables selon les climats.

La molécule est atteinte. PASTEur en fournit une magnifique démonstration par la combinaison d'une base active, la cinchonine, avec l'acide tartrique droit, soumise à l'action de la chaleur ; les différences de solubilité des produits permettent de séparer l'acide tartrique gauche et, par l'addition des deux, Pastrur crée l'acide racémique. Il a réalisé une crise de mutation dans l'espèce. De même, les botanistes ont constaté que l'hybridation, suivie de sélections ou de ségrégations sous des climats divers, donnent des petites espèces nouvelles et stables: l'Aegilops spaeltaeformis de Jordan, issu du croisement de l'Aegilops ovata et du Blé, est un équilibre vivant, fixé, contrôlé durant quarante géné. rations (1).

Avant de passer à l'étude des germes, je cite les expériences de Standfuss et de Fiscuer sur les papillons et mes propres recherches sur le Maïs, comme preuves d'altérations profondes produites par l'action brutale du milieu sur un matériel plastique. Les mutations obtenues, comme celles de l'acide tartrique droit dans les expériences de PASTEUr. comme celles de l'Enothera Lamarcliana de M. DE VRIES, n'apparaissent pas isolées, mais par groupes. Il y a, dans la pulvérisation de l'espèce, plusieurs états d'équilibre possibles, qui ne sont pas quelconques et qui se forment simultanément.

La notion de germes eristallins offre un grand intérêt. En 1866, Gernez, préparateur de Pasteur, découvre un pro-

1. - L. Blaringhem: Les problèmes de l'Hérédité expérimentale, Paris, Flammarion, 1919, chap. III. 
cédé nouveau, très simple, pour séparer les tartrates droits et les tartrates gauches dans la solution sursaturée de racémate de sonde et d'ammoniaque ; un fragment aussi petit que l'on voudra, mais cristallisé droit, fait déposer le tartrate droit, laisse le tartrate gauche en totalité dans l'eau-mère. Les germes cristallins agissent, d'après GERnEz, sur les solutions sursaturées au même titre que les ferments; comme eux, ils flottent dans l'air commun des laboratoires ; l'air filtré et l'air calciné ne font plus eristalliser les solutions sursaturées (1867).

Les solutions de sels susceptibles d'être maintenues en état de sursaturation se comportent donc comme les éléments femelles dans l'attente de l'unique anthérozoïde, de l'unique grain de pollen nécessaire, et suffisant, pour constmuire un embryon (Thuret, 1845 ; Naudin, 1863).

Le germe eristallin n'a d'activité que sur les solutions sursaturées de son espèce; alors, il féconde sans déchet. Avee un seul cristal-germe de formiate de strontiane, GERNEz (1868) épuise la solution inactive de ce corps, et, selon le cristal ensemencé, hémièdre droit ou hémièdre gauche, la fait cristalliser en totalité en formiate droit ou en formiate gauche ; il fournit ainsi une démonstration très élégante du fait découvert par PASTEUR quinze ans plus tôt, à savoir, que chez le formiate de strontiane, comme chez le quartz, la dissymétrie n'est qu'apparente ; elle affecte l'arrangement des molécules et non la molécule elle-même, car les solutions sont inactives. Enseignement fécond pour les biologistes : croisons les formes critiques avec leur souche supposée; l'absence de déchet dans la fécondation et l'absence d'avortement dans la descendance donnent la preuve de l'identité spécifique. Cette règle fut énoncée par NaudrN en 1858 et j'ai donné de nombreuses preuves de sa validité. 
Les équilibles des substances dimorphes, étudiés aussi par Gernez (1877), fournissent des critères aussi précieux de la spécéité ; leurs états sont ceux des adaptations doubles: le froid, le frottement déterminent dans leurs solutions des équilibres analogues, quant à l'aspect, à ceux qui résultent de l'apport des cristaux-germes; les formes seules sont ici encore en jeu ; l'espèce, la molécule n'est pas altérée. Les dégénérescences héréditaires des Céréales, des Lins, dues au climat, rentrent dans cette catégorie de phénomènes.

En 1865, Pasteur étudie la maladie du Ver à soie, putréfaction toujours accompagnée de corpuseules qui passent du corps de l'insecte dans les œufs. Il devine que les corpuscules, inorganisés ou vivants, sont des germes ; il suffira de découvrir un couple de papillons privé de ces germes pour obtenir une descendance débarrassée de la pébrine. Sous sa direction, Gernez (1866) prouve la nature vivante du corpuscule; Pasteur résiste, puis s'enthousiasme à cette idée. Il vient de découvrir un exemple précis d'une maladie transmise par l'œuf, d'un parasite si intimement associé à son hôte que leurs germes s'interpénètrent et constituent des complexes vivants l'ésistant à la reproduction sexuée. Exemple saisissant de ces adaptations si curieuses que présentent les Rouilles et les Charbons intimement associés aux Céréales, que présentent sans doute aussi certaines variétés de plantes panachées qui sont des mosaïques héréditaires, et divers cancers végétaux.

Par ses découvertes, Pasteur a justifié sans s'en douter l'hypothèse de la Pangénèse, énoncée timidement par DARwin exactement à la même date (1868) ; les naturalistes d'alors ont refusé d'y accorder la moindre attention ; cette étude contribuera peut-être à les engager dans une voie qui 
sera certainement féconde s'ils utilisent les modes de raisonnement et les techniques si perfectionnées de l'école pastorienne (Travaux de Noel Bernard, 1902-1911).

$$
\text { *** }
$$

Les études de Pasteur sur les ferments, sur les maladies microbiennes, sont trop connues pour qu'il soit utile de les résumer ici. E. Duclaux, M. le docteur Roux ont exposé, avec la plus grande clarté, les conceptions de celui qui les a choisis pour collaborateurs et leurs résultats.

An profit de ceux qui étudient la variation et l'hérédité, tant an point de vue pratique que théorique, je veux cependant dégager de cette série de recherches, suite logique des découvertes précédentes, une notion essentielle.

Pasteur n'examine plus désormais que les réactions des fragments de l'individu et non le polymorphisme propre à l'espèce. Parce qu'il limite rigoureusement le sujet, sa technique atteint la perfection et soll raisonnement présente une rigueur impeccable.

Les germes les plus divers flottent dans l'air commun, abondent dans les poussières déposées à la surface des liquides ou des solides ; certains vivent constamment à l'abri de l'air, dans la profondeur des tissus ; d'autres se multiplient à l'air et en profondeur. D'où leur polymorphisme, les variations dans leur activité, les modifications de leurs aptitudes selon les circonstances.

La lutte pour la vie, pour l'oxygène, pour l'aliment convenable, n'apparaît nulle part dans la nature 'mieux que dans les ballons de lait, de jus du raisin, de moût de bière; la pureté du ferment, son homogénéité, son développement libre sans aucune gêêe, à l'aide d'une nourriture appropriée, 
telles sont pour Pasteur les conditions essentielles d'une bonne fermentation. Guidé par cette conception, il perfectionne sa technique chaque fois qu'une objection se pose ; il montre aux naturalistes sceptiques l'absence de générations spontanées, l'apport nécessaire des germes extérieurs, les conséquences de leur ségrégation. Darwin a fait une longue croisière de six années anx îles volcaniques des Galapagos, surgies des profondeurs du Pacifique, pour observer, avec moins de garanties, les faits essentiels de sa théorie du transformisme ; Pasteur crée une technique précise, simple et sûre, qui permet d'assister en quelques jours, dans le laboratoire et presque sans frais, à la ségrécation des ferments, des microbes, qui se comporteront de toute autre façon dans ses ballons que dans la nature.

La plasticité des microorganiques paraît beaucoup plus grande que celle des organismes supérieurs. Pour conserver intacte la notion de spécificité, si claire en cristallograplie et en chimie, Pasteur déploie une ingéniosité qui crée les méthodes parfaites; sompçonnant une erreur dans la métamorphose, constatée maintes fois dans ses ballons, du mycoderme du Vin en levure de Bière, il imagine la technique des cultures pures.

Depuis vingt ans, nos études sur le perfectionnement des Céréales reposent sur la préparation des lignées pures. Je montre comment les règles de la ségrégation rapide des aptitudes individuelles, précisées par le biologiste danois W. Johannsen (1902) et la sélection méthodique de sortes remarquables de Blés, d'Avoines et d'Orges, inaugurée par le suédois N. Hualaiar Nilsson (1895), dérivent directement des travaux de Hansen, qui applique à la lettre les principes et les procédés décrits dans les Etudes sur la Bière (1873).

La culture prure dn Bacille du Choléra des poules 
nous conduit à une autre conséquence. L’individu, par sa multiplication en conditions définies, par son propre vieillissement, devient son propre vacein ; la virulence atténuée par l'oxygène est une modification réversible analogue en tous points aux modifications réversibles, elles aussi, des Céréales, des Lins, sous divers climats. Nous voilà outillés pour l'étude précise de l'hérédité acquise.

La réversibilité de l'atténuation est examinée sous toutes ses formes, dans toutes ses causes, sur la Bactéridie charbonneuse pal Pasteur, Chamberland et Roux; elle présente'les caractères des sélections perfectionnées de la Betterave à sucre, et le seul rapprochement de ces faits suggère des méthodes plus sûres peut-être que celles qui sont couramment adoptées pour obtenir les meilleurs rendements industriels. Il arrive ici que la Bactéridie, qui d’ordinaire sporule, perd dans des condlitions déterminées, diverses d'ailleurs, la faculté de domner des spores. Voilà, nous dit-on, un procédé de fabrication artificielle de races ; mais, parce que la perte est progressive, graduée et, jusqu'à une certaine phase-limite, réversible, PAsteur et ses disciples ne doutent pas qu'ils aient affaire à ces individus incomplets, juvéniles, comparables aux insectes parthénogénétiques, aux sortes d'arbres fruitiers parthénocarpiques, qui, ne donnant plus de graines, disparaissent tôt ou tard, à moins que l'homme ne les sauve par des soins d'autant plus délicats que la propagation asexuée se prolonge davantage. C'est l'état des Kakis japonais, des Bananiers, celui des Cannes à sucre, état auquel il est très difficile de remédier, bien que l'avenir des plantations soit en jeu. 


\section{***}

L'œuvre de Pastreur n'est qu'une suite remarquable d'expériences sur les phénomènes les plus intimes de la vie actuelle. Par ses études sur les cristaux, il nous montre la trame de tout ce qui constitue la stabilité de l'espèce, la permanence des germes. Par ses études sur les ferments, il nous découvre la plasticité de l'individu, la souplesse de la vie cellulaire dans ce qu'elle présente de plus profond et de plus caractéristique.

Sans doute, par ce côté particulier, il fournit à la thèse de Darwin des arguments décisifs. Dans la nature, la vie détruit la vie, lutte pour la place qui explique la distribution des êtres. L'aptitude à se plier aux circonstances, à conserver une empreinte durable d'adaptations passagères, est aussi mise en valeur ; c'est l'hérédité acquise. Enfin je trouve, le premier sans doute, dans la transmission des corpuscules de Pébrine par l'œuf du Ver à soie, la meilleure prenve de la possibilité d'une altération, par un apport extérieur, des germes eux-mêmes, conséquence à laquelle DARIvin est conduit par l'analyse des faits rassemblés daus les deux ouvrages qui sont les fondations mêmes de sa doctrine du transformisme.

Toutefois, Pasteur démontre, en même temps, que ces modifications et altérations ne changent pas, en définitive, la nature de l'espèce, pas même celle de la race. Sans doute des variations notables peuvent être régulièrement transmises des cellules-mères aux cellules-filles, d'un papillon aux œufs et à quelques descendants. Mais le transformisme, tel que le conçoivent les naturalistes et les philosophes, se limite-t-il à des altérations passagères, superficielles, accidentelles ?

En établissant un parallélisme entre les modifications des 
individus d'ordre supérieur, dont la cloissance est régie par la coadaptation dess organes et les dimensions limitées, avec les modifications subies par los cristaux et les microorganismes qui transforment identiquement à eux-mêmes des masses pour ainsi dire illimitées de matériaux, je me suis demandé si je n'allais pas détruire l'illusion de ceux qui eroient étudier, et même provoquer expérimentalement, l'essentiel du transformisme. Or, je viens de découvrir, par la lecture des cuvres de Pasteur, que la forme n'est pas l'espèce; elle n'est qu'une des multiples manifestations de l'espèce. Depuis ringt ans, tous mes efforts tendent à apporter à la consolidation du dogme des transformistes quelques matériaux solides : je constate la possibilité de provoquer du polymorphisme héréditaire dans les limites mêmes de 1 'espèce.

Et cependant, quelles perspectives pour l'avenir prochain des civilisations ! Sans doute, l'espèce des naturalistes, des philosophes, n'est pas altérée dans son essence, mais nous savons trier les variations individuelles, en découvrir les causes immédiates, les provoquer à volonté. Nous savons domestiquer ces créations, les produire en nombre illimité, identiques à elles-mêmes, pour les mettre en l'état convenable à la disposition des ferments eux-mêmes domestiqués ou de machines plus exigeantes encore. Avec la technique des lignées pures s'évanouissent la plupart des preures en faveur d'une doctrine philosophique relative à l'origine et à l'avenir du monde organisé; mais le transformisme expérimental devient me science propre; il donne les sécurités des sciences physiques et chimiques; il développera dans un domaine immense, où l'homme est compris, les perfectionnements acquis dans la domestication des ferments et des microbes patlogènes sous la ferme surveillance de PAsteur. 
Pasteve a entrevu une fissure dans le bloc mystérieux de la vie et nous pouvons l'y suivre avec précaution.

Est-il vrai que tontes les dissymétries moléculaires sont dérivées ou procèdent des êtres vivants ?

" Messieurs, dit Pasteur en 1883 (1), nune particularité singulière concerne la dissymétrie moléculaire. On trouve la dissymétrie établie dans un très grand nombre de prin. cipes immédiats des animaux et des végétaux, notamment dans les principes immédiats de la vie. Tous les produits, pour ainsi dire, de l'œuf et de la graine sont dissymétriques... Sans nul doute, je le répète, si les principes inmmédiats de la vie sont dissymétriques, e'est qne, à leur élaboraration, président des forces eosmiques dissymétriques ; c'est là, suivant moi, un des liens entre la vie à la surface de la terre et le cosmos, e'est-à-dire l'ensemble des forces répandues dans l'univers... Que faut-il faire pour imiter la nature? Il fant rompre avec vos méthodes (2), qui sont à ce point de vne surannées et impuissantes. Il faut ehercher à faire agir des forees dissymétriques, recomrir à des actions de solénoïde, de magnétisme, de mouvement dissymétrique luminenx, à des actions de substances elles-mêmes dissymétriques. "

En fait, PASTEUR a préparé un corps ganche à partir d'un corps inactif par l'intermédiaire d'une substance elle-même active, la cinchonicine, et il insiste sur ce que les forces mises en jeu ici diffèrent de celles de la chimie ordinaire et se rapprochent de celles auxquelles est sommise la nature végétale.

1. - La dissymétrie moléculaire. Conférence faite à la Société chimique de Paris, le 22 deecmbre 1883.

2. - Dissolvauts, température, pression, qui mettent en jeu des forees symetriques. 
Il termine sa conférence par une invitation qui, à ma connaissance, n'a pas été suivie : "Pour faire les produits essentiels de la vie, les principes immédiats de notre sang, principes qui doivent être mous, flexibles, glissants, non cristallins, la nature, pour faire ces produits de la vie, n'aurait qu'à unir un nombre minimum de trois groupes dissymétriques. "

PASTeur n'était done pas de parti pris opposé à la génération spontanée, ni davantage à la métamorphose des espèces les unes daus les autres. Il reconnaît qu'au point de vue expérimental la combinaison seule de substances dissymétriques a permis l'altération de la dissymétrie moléculaire. Par imitation, nous serons tentés de ne trouver de créations nouvelles, d'espèces nouvelles, que dans les productions hybrides, revenant ainsi au point de départ, entrevu, mais non démontré par Linsé.

La notion de transformisme renferme quelque chose de plus ; elle doit rendre compte de cette hiérarchie des formes, de cette subordination des caractères sur lesquelles repose la méthode naturelle de Bernard de Jussieu. Or, ici il ne s'agit plus de dérivation immédiate, constatée, mais de liaisons par analogie, par rapprochement. Ici, interviennent l'espace et le temps.

Lorsqu'un naturaliste digne de ce nom dispose côte à côte, à l'état vivant (Jordan, G. Allard), ou, s'il ne le peut, en matériaux secs (DE Candolle, Giraudias), des formes qui offrent des caractères indiscutables de parenté, il fait, sans le vouloir peut-être, du transformisme. C'est une histoire logique, qui n'est peut-être pas vraie, mais qui offre pour l'esprit humain en l'état actuel de son développement les plus grandes chances de s'être prodnite ; c'est l'histoire, dis- 
je, des métamorphoses réalisées au cours de milliers et même de millions de siècles à partir d'une souche, ou de plusieurs souches primitives.

Si la souche a pour point de départ la combinaison d'une matière dissymétrique à une antre matière dissymétrique, il est infiniment probable, Pasteur nous l'apprend, que cette combinaison, cet accident initial n'a pas donné une seule catégorie de groupements, mais quatre, huit, peut-être seize groupements fondamentalement distincts. Pourquoi vouloir, alors que rien ne nous impose cette condition, édifier une histoire généalogique de l'ensemble des êtres vivants, ou même de l'ensemble des êtres d'un même règne, sur un unique point de départ?

N'est-il pas plus commode, pour approfondir le problème, et surtont plus vrai, de recomnaitre qu'il y a un abîme entre les Bactéries et les Mousses, moindre pent-être entre les Gymnospermes et les Angiospermes? Chaque embranchement, chaque classe a son transformisme propre, avec ses règles particulières, ses liaisons plus ou moins solides. Ces suggestions sont acceptées en fait par les naturalistes; elles les ont conduits à la découverte de lois de la croissance applicables à chaque système; les philosophes ne reulent pas tenir compte des embranchements, des classes.

L'expérimentation, chère à PASTEUn, ne parait pas devoir nous aider dans ces reconstructions, du moins dans un avenir prochain. Pour édifier des transformismes historiques, il faut, de toute nécessité, tenir compte de la durée, facteur qui nous échappe, et, pour cette raison, nous pouvons seulement attendre de ces efforts des satisfactions intellectuelles légitimes, mais pen on pas d'applications véritables au progrès de l'humanité. 


\section{TABLE DES MATIÈRES}

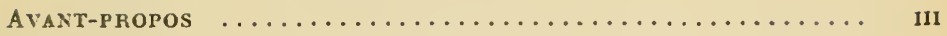

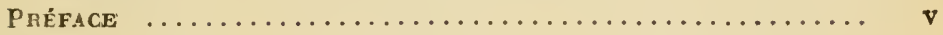

LIVRE PREMIER

LES VARIATIONS DE L'ESPÉCE

LA NOTION D'ESPÈCE...........

L'espéce de Lixié et la nomenclature binaire, 1 ; permanence supposée des hybrides, 3. - Méthode naturelle de Bernaro oB Jussıev, 4; Subordination des caractères, 4; Adaptation à la Chimie par La roısıer, 5. - Molécule intégrante des cristallographes...

Espèces jordaniennes de la Pensée sauvage, 8 ; séries paralléles et découverte des petites espéces par analogie, 10 ; vérification par le semis de la constance des caractères ..................

L. Pasteur fait l'étude sériée des tartrates, 14; découvre une particularité des substances dimorphes, 16 ; définit la variété, 17 . Dissymétrie moléculaire; hémiédrie des tartrates et polarisation rotatoire, 18. - Paratartrates inactifs par mélanges en quantité égale de cristaux droits et de cristaux gauches, 19. - Permanence de la dissymétrie moléculaire dans les substitutions chimiques, 21 ; définition de l'espèce (Chevreul, 1823, Pastevr, 1860)..........

Fréquence de la dissymétrie moléculaire caractérisée par la polarisation rotatoire, 23. - Les produits naturels sont dissymé- 
triques, les corps artificiels ne le sont pas, 24. - Sous-espèces définies dans l'espèce chimique. .........................

\section{CHAPITRE II}

\section{LA VARIATION DES FORMES}

Enchatuement des recherches de Pasteur sur les cristaux, 27. Définition généralisée de la dissy métrie moléculaire, 28 ; elle n'exclut pas la formation de cristaux homoèdres; influence du milieu. ....

Adaptations doubles de la Renouée amphibie, 30 ; variations saisonnières et sortes locales de Papillons, 32 ; mosaïque des formes du Lierre, 34. - Polymorphisme des malates et des tartrates cristallisés........................................

Formes-limites des cristaux du Soufre, 36 ; elles dépendent des conditions de cristallisation, 37 ; l'une est plus stable que l'autre, 38. - Variétés instables de Pavot : variations sexuelles dépendant de la nutrition, 40 ; relation entre la couleur des fleurs et la succession des phases sexuelles, 42. - Isodimorphisme des chlorures de naphtaline et de naphtaline monochlorée, 43. Chimẻres végétales de Morelle et de Tomate..................

Symétrie végétale et excitation inhibitoire d'aprés Errera, 46. Expériences de PASTeur sur la régénération des cristaux, 47. Róle des impuretés dans la croissance et le polymorphisme, 50. Les stabilisations des formes cristallines et des formes vivantes ne diffèrent que par la durée $\ldots \ldots \ldots \ldots \ldots \ldots \ldots \ldots \ldots \ldots \ldots . . . .$.

1 CHAPITRE III

\section{HÉRÉDITÉ, HYBRIDATION ET MUTATION}

Eléments stables de l'espéce et anomalies, 57. Conception de Pastevr relative à l'hérédité, 59 ; permanence de l'hémiédric de l'acide tartrique après substitutions de bases donnant les composés isomorphes, 60. - Comparaison de ces phénomènes avec l'hérédité

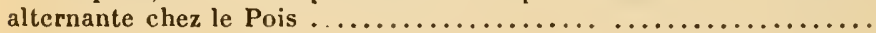

Degrés de spécićïté d'après Naunis, 63. - Enchainement des espéces, des genres, des familles et arbrc généalogique, 65. - La notion de transformisme dérive des notions de temps et d'espacc, 66 ; elle ne s'applique pas en chimie, ni dans la science des microbes, 66 ; la génétique expérimentale se rattache par son objet et ses procédés aux sciences chimiques et microbiologiques ....................

Les hybrides sont des mosaïques vivantes d'essences spécifiques tendant à se séparer, 68; régles des retours de Naunis, 68; instabilité des hybrides mixtes, 69. - Mosaïques cristallines de 
Bitartrate d'ammoniaque et de Bimalate d'ammoniaque; instabilité de ces édifices moléculaires d'après Pastrur, 70. - Ebranlements moléculaires résultant de la combinaison d'espèces distinctes actives ; acide tartrique et asparagine, Seigle et Blé, 74. - Transmutation de sous-espéces actives l'une dans l'autre; pas de régles, mais jeu

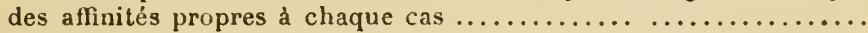

Mutation des espéces et acide racémique, 76 ; production simultanée de plusieurs sous-espèces, 78. - Expériences de Srandfuss (1900), de Fischer (1907), sur les mutations provoquées par la congélation des chrysalides de Papillons, 79. - Expériences de Brarivghrm (1907), sur les traumatismes et les mutations du Maïs...........

La mutation entraine la pulvérisation de l'espèce, 84; Assemblages possibles des dissymétries moléculaires combinées deux par deux, trois par trois, etc..., et définition de la matièré vivante par

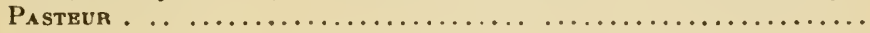

\section{LITRE II}

\section{LES GERMES}

\section{CHAPITRE IV \\ LE ROLE DES GERMES}

"Lavie, c'estlegerme; le grerme, c'est la vie "(PAsteun, 1884), 89.Séparation des tartrates droits et gauches dans les racémates, par les germes cristallins dans les solutions sursaturées (GrRnez, 1866), par le ferment tartrique (PAstbun, 1857), par l'intermédiaire d'une

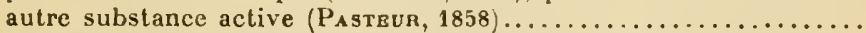

Spécificité des germes cristallins, 95 ; la chaleur les détruit, l'air les transporte, 96 ; concordance des découvertes de GBRnzz et de

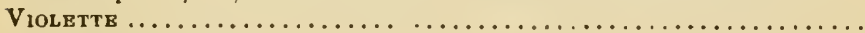

Sexualité végétale, 99 ; transport du pollen par l'homme, par le vent, 100. - Expérience de Thuret (1845) sur les Fucus, 101. Un seul grain de pollen est nécessaire pour donner une graine

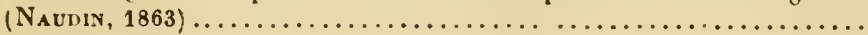

Suite des expériences de Gervez (1866 1877) sur la sursaturation, 103. - Epuisement d'une sulution de formiate destrontiane par un cristal droil ou par un cristal gauche, 10ई; opposition de la dissymétrie 
moléculairc et de ia dissymétrie dans l'arrangement des nulécules, 105. - Substitution dune variété de Tabac à une autre par des pollinations répétées, $100^{\circ}$ cas des variétés de Maïs ................

Etats instables des substances dimorphes et adaptations, 110 ; ici le germe peut être remplacé par des agents physiques, le froid,

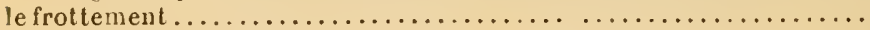

\section{LE MILIEU ET LA MIGRATION DES GERMES}

Relations entre le milieu etles espèces. - Peuplement de l'archipel volcanique des Galapagos d'après DARwis, 115; origines américaines de la faune et de la fonce, 117 ; ségrégation et substitution

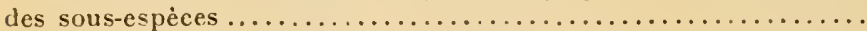

Lalcool amylique emprunte son pouvoir rotatoire à des germes vivants (PAsteur, 1855). 120 ; découverte du ferment lactique (1857), 122. - Nature et conditions d'une bonne fermentation, 123 ; agents qui facilitent la substitution des ferments, 124. - Aliments de choix pour la levure, 124; milieux de culture d'origine purement minérale, 127. - Rôle des infiniment petits chimiques dans

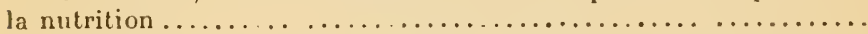

Les grermes-ferments sont dans l'air commun, 129 ; l'air caleiné en est privé, 130. - Negnham el Srarlanzayi discutent la sénération spontanée, 131 ; rôle de l'oxygène dans les fermentations d'après

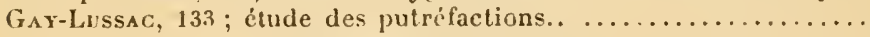

Pasteur nie la rénération spontanée, 131; prouve que l'air apporte les corpuscules-germes, 136. - Méthodes rigoureuses de préparation de milieux de culture stérites. 138 ; perfectionnements

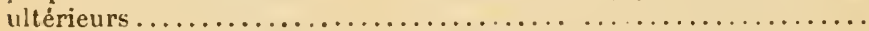

\section{CHAPITRE VII}

\section{ADAPTATIONS RÉCIPROQUES DES GERMES ET DES PARASITES HERÉDITAIRES}

D. GenNez conseille la lecture des litudes sur la maladie du Ver à soie, par Pastevn, 141. - Progrés de l'étude de la Rouille des Céréales, 142 ; cycles des prassages du parasite sur des végétaux différents, 143. - Races physiologiques de Ronilles, 145 ; hypothèse

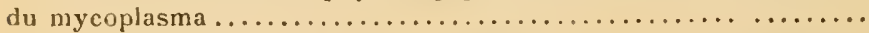

Caractères généraux de la maladie du Ver à soie, 146 : pébrine et corpuscules, 147. - PAstelr, envoyé en mission à Alais, 150 ; énonce les règles qu'il faut suivre pour ol,tenir de la graine saine, 151. 
Méthode de sélection généalogique ou pédigrée, 151.- Origines des pontes saines et procédés de cuntròle .....................

153

Pastrur admet d'abord que le corpuscule est un produit de dégénérescence de l'organisme, 15\%; expériences de contamination réalisées à Valenciennes par Grrvez, 15j; adhésion tardive, mais formelle, de Pasteur à la preuve que le corpuscule est un microorganisme vivant, 156. - La pébrine du Ver à soie est le premier exemple bien étudié de malaclie à la fois parasitaire et héréditaire.

Plantes à panachure infectieuse communiquée par la greffe, 157. Mosaïque des tissus albinos et des tissus à chlorophylle, 158 ; diversilé des modes d'hérédité de la panachure, 158. - Le cancer (Crown-gall) des Chrysanthémes est dû à une bactérie, 160 : discussion actuelle et méthodes à suivre pour la résoudre.........

Deux maladies héréditaires, la pébrine et !a flacherie, affectent indépendamment les Vers à soie, 162 ; diversité des symptômes, 162 ; diversité des parasites, 161. - Le Nosema bombycis, cause de la pébrine, est in sporozoaire hautement différencié. ............

Hypothèse de la pangénése de Darwix vírifiée dans un cas par-

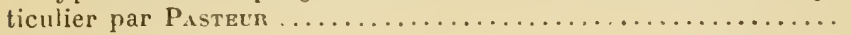

\section{LES MODIFICATIONS DE L'INDIVIDU}

CHAPITRE VII

\section{PLASTICITÉ DES FERMENTS FIGURÉS ET LIGNEES PURES}

Etude de la fermentation gallique par vas TIEgurm, 169. Modes de vie du Penicillum et de l'Aspergillus, agents de cette fermentation, 171; leurs formes aériennes el leurs formes ferments sont des adaptations doubles, 172. - Polymorphisme du Mucor

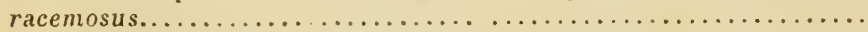

Pasteur publie sesétudes sur le Vin (1866), sur la Bière (1873), 176 ; variations de formes et d'activité des ferments avec la quantité d'oxygènc, 177. - Métamorphose présumée des microorganismes ferments, 178: substitution de plusieurs levures an cours de la

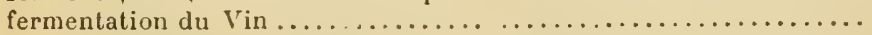

Individualité des levures accusée par leur domestication, 180. - 
Notion de lignée pure dans les Orges de Brasserie, 183 ; lignées pures homogènes et lignées pures instables..................

Méthodes de préparation des lignées pures de levure, 187. Application à l'étude des variations dues au milieu, 189. - Préparation de races asporogènes, 192 ; elles sont réversibles..........

Formes végétatives à fécondité limitée par la vieillesse, 195 ; formes juvéniles et pradogénèse $\ldots \ldots \ldots \ldots \ldots \ldots \ldots \ldots \ldots \ldots \ldots \ldots$

\section{ADAPTATIONS RÉCIPROQUES ET VACCINS}

Mutation supposée de la fleur du Vin en levure, 196; découverte des circonstances de contamination et des moyens de l'éviter, 197 ; Pastbur affirme qu'il n'y a pas de métamorphose spécifique........

Réflexions de PAsterr sur le vaccin de JENNer; sur la virulence de la Bactéridie charbonneuse, 199. - Etude du Choléra des poules, 201 ; il est dû à une bactéric. Milieux de culture très favorable ou toxique, réaetifs de la pureté, 202. Diminution de la virulence et

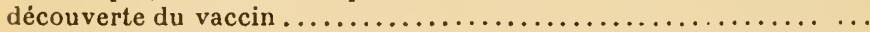

Prétendue hérédité acquise: adaptations transmises des Céréales cultivées sous différents climats, 204; adaptations sexuelles au milieu, 206: leur réversibilité et étude critique d'aprẻs Gatton ....

Pasteur analyse le mécanisme de la préparation du vaccin du Choléra des poules, 208 ; altérations des milieux de culture, 209; immunité humorale, 210. - Mécanisme de la résistance au parasile atténué, 211. - L'oxydation est la cause immédiate de l'atténuation, 214; prolongée, elle tue le microbe. - Variétés de Choléra découvertes chez les Gallinacés, 218 ; elles n'ont pas de relation immédiate avec le Choléra des poules ..........................

\section{CHAPITRE IX}

\section{LUTTE POUR LA VIE, SÉLECTION ET CARACTÈRES ACQUIS}

Maladies charbonneuses, 220 ; Davaine découvre la Bactéridie; sa présence nécessaire et suffisante pour déterminer le sang de rate, 221. - Conditions déterminant la sporulation d'aprés Kосн, 223. Pastrun el Joubert découvrent la résistance des spores à l'alcool, à l'oxygène comprimé, 224; faible résistance de la Bactéridie au manque

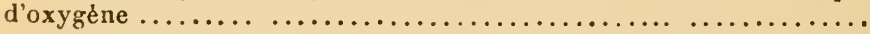

Discussion de Pasteur avec Colin, 226. - Lettre de Pasteun a H. Bovlex, relative au rôle unique de la Bactéridie; preuve de la dilution, 228 ; le sang privé de Bactéridie ne donne pas le charbon, 


\section{Pages}

229, - Entrainement de Pasteru pour les f́ludes vétérinaires, 231. - Eliologie du charbon . . . . . . . . . . . . . . . . . . .

Résultats acquis en 1880, 233. Craduation de la virulence el réversibilité de lalténuation, 23:. - Préparation de vaccins d divers degrés d'atténuation; eomparaison avec la śllection de la Betterave ̀̀ sucre, 235. - Attínuation stabilisée de la bactéridie charbonneuse d'après M. Roux, 237. - Les 1'aces asporogènes sont des morcellements d'individus, au même titre que les Bananiers, les liakis. les Cannes a sucre; ce ne sont pas des mulations ... . . . . . . . .

CONC:L, ISIONS

liésumé el Conchusints. 




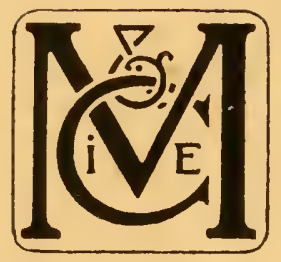

Prix Fr. net

L 7214 





